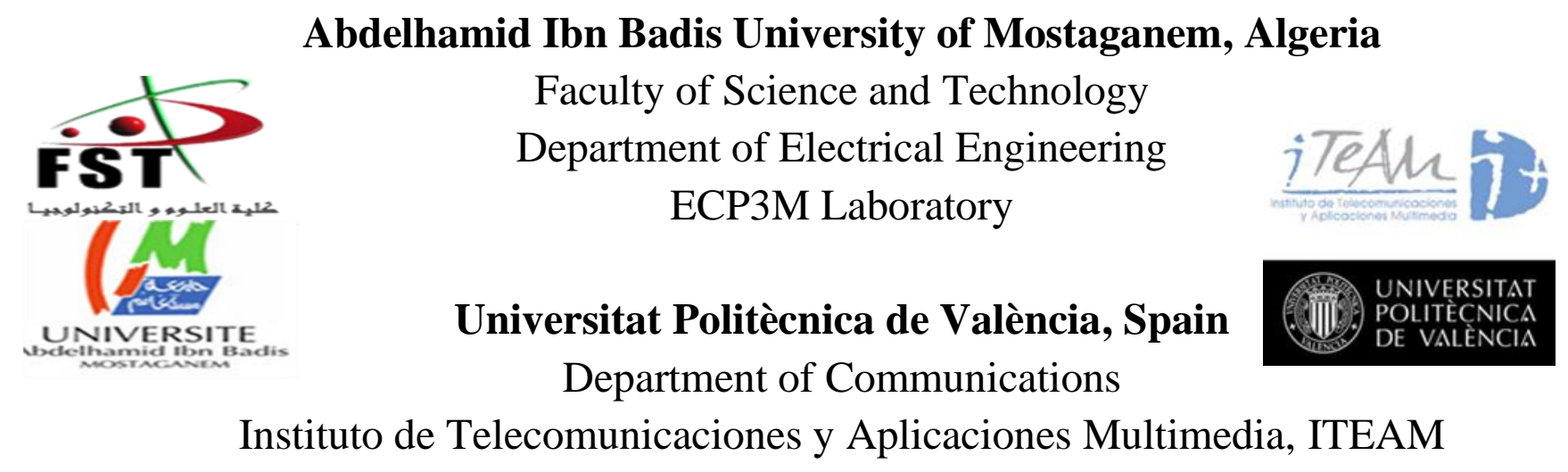

\title{
THESIS
}

To obtain the degree of

Doctorate in Optoelectronic Materials and Energy

\section{Design of communication systems based on broadband sources for fiber and free space optical links}

\section{Presented by Imene SEKKIOU}

Supported in May 2021 in front of the jury:

President

Said Meskine

Reviewers

M.Ali Khalighi

Gema Piñero

Ali Djebbari

Thesis co-supervisors

Hadjira Benoudnine

Beatriz Ortega Tamarit
Associate Prof

Associate Prof

Prof

Prof

Prof

Prof
Abdelhamid Ibn Badis University, Mostaganem, Algeria

Ecole Centrale Marseille, Marseille, France University of Polytechnic of Valancia, Spain Djilali Liabes University, Sidi Belabes, Algeria

Abdelhamid Ibn Badis University, Mostaganem, Algeria University of Polytechnic of Valancia, Spain 
It is not easy to complete a doctoral thesis. So, in order to withstand the moments of pressure and doubt, it is necessary to know how to surround oneself and find an attentive ear. I would therefore like to express all my gratitude to the people who have supported me, encouraged me from near or far, consciously or unconsciously.

This thesis work was carried out in the Electrical Engineering Department within the Laboratory of Elaboration and Physico-Mechanical and Metallurgical Characterization of Materials (ECP3M), Abdelhamid Ibn Badis University, Mostaganem, Algeria in conjunction with the Communications Department within the Institute of Telecommunications and Multimedia Applications (ITEAM), Polytechnic University of Valencia, Spain.

My greatest gratitude goes to my supervisors Prof. Benoudnine Hadjira and Prof. Beatriz Ortega Tamarit for making this $\mathrm{PhD}$ project possible. Thank you for your trust, and thank you for the advice which is always very accurate, not necessarily pleasant to hear, but which has always pushed me to do better and to surpass myself.

I am very grateful to the members of the committee of my thesis jury for their time and dedication. Thank you to Dr. Said Meskine from Abdelhamid Ibn Badis University, Mostaganem, Algeria, Dr. M.Ali Khalighi from Ecole Centrale Marseille, France, Prof. Ali Djebbari from Djilali Liabes University, Sidi Belabes, Algeria and Dr. Gema Piñero from University of Polytechnic of Valancia, Spain, for the interest they showed for my research work and for agreeing to be examiners to evaluate my doctoral thesis.

I would also like to thank all the persons of the laboratory ECP3M and their head Prof. Boukortt Abdelkader for his precious help, also thanks to my colleagues in ITEAM and especially the Research Group PRL (Photonic Research Laboratory). Special thanks to Dr. José Mora at the Universitat Politècnica de València, Spain and Dr. Francisco I. Chicharro for their help.

I must also thank my family members for their continued support, in particular, my father (peace be upon him). All my efforts to complete this doctoral thesis are dedicated to him. To my mother, brother Mahmoud, my husband Hamid Zenfoukh, my dear grandmother (peace be upon her soul) and my uncle Kredda Ghaouti for their dedication and permanent help.

Good reading to the few brave people who will read the rest of this manuscript... 
Theory is when you know all and nothing works.

Practice is when all works and nobody knows why.

In this case we have put together theory and practice: nothing works... and nobody knows why!"

$\underline{\text { Albert Einstein }}$ 
Optical wireless communication (OWC) is a very promising technology for future wireless communications developments. It has attracted increasing interest from researchers and several companies around the world are currently working on the development of very highspeed wireless networks. The scientific and industrial communities believe that OWC will be a complementary technology in its various forms: Free Space Optical communications (FSO), Visible Light Communications (VLC), Light Fidelity (Li-Fi).

In fact, the optical spectrum has been considered for many years as a great opportunity for wireless communications especially due to the saturation of the radio frequency (RF) spectrum.

This dissertation deals with the use of broadband sources in visible light transmission systems (VLC) as well as fiber optic systems. To carry out the research, three parts can be distinguished:

- In the first part, we consider the study and simulation of Light Emitting Diode (LED) components with the WIEN2k software by focusing on the optical and electrical properties of elements $I I-V I$.

- The second part deals with the design, implementation and testing of different VLC communication prototypes for analog and digital transmission in simplex and halfduplex mode. We have demonstrated that an OWC system using a broadband source (i.e. an LED) can be used not only for data transmission, but also for wireless power transmission. Moreover, the synchronization problem and the detection of level " 1 " or " 0 " of a bit often arise in the optical wireless communication systems. This is a result of the attenuation nature of the light over the distance and the problem of Non LineOf-Sight (NLOS) between the emitter and the receiver. To deal with this problem, a communication protocol ensuring reliable digital data transmission with an adaptive bit level detection algorithm has been provided and its effectiveness has been demonstrated by the transmission of texts and images.

- In addition, this thesis provides a solution for the implementation of wavelength division multiplexed - orthogonal frequency division multiplexed (WDM-OFDM) transmitters based on the use of broadband sources in the infrared spectrum for centralized bidirectional fiber networks. Despite the chromatic dispersion that avoids the use of this type of optical sources, the inclusion of certain structures before detection allows the transmission of OFDM signals in optical links. Carrier reuse, dynamic bandwidth allocation and multiband OFDM signals transmission will be experimentally demonstrated by using optical broadband sources in WDM networks.

The main results obtained during this thesis work demonstrate the study procedures, for each part, the effectiveness of the proposed solutions as well as the constraints encountered.

Keywords: OWC, VLC, FSO, Fiber, Light, Broadband sources, LED, Optical modulation, Emitter, Receiver 
La communication optique sans fil (OWC) est une technologie très prometteuse pour les futurs développements des communications sans fil. Elle a suscité un intérêt croissant de la part des chercheurs et plusieurs entreprises du monde entier travaillent actuellement au développement de réseaux sans fil à très haut débit. Les communautés scientifiques et industrielles pensent que l'OWC sera une technologie complémentaire sous ses différentes formes : Free Space Optical communications (FSO), Visible Light Communications (VLC), Light Fidelity ( $\mathrm{Li}-\mathrm{Fi})$. En fait, le spectre optique est considéré depuis de nombreuses années comme une grande opportunité pour les communications sans fil, notamment en raison de la saturation du spectre des radiofréquences (RF).

Cette thèse porte sur l'utilisation de sources à large bande dans les systèmes de transmission de la lumière visible (VLC) ainsi que dans les systèmes à fibres optiques. Pour mener à bien la recherche, on peut distinguer trois parties :

- Dans la première partie, nous considérons l'étude et la simulation de composants de diodes électroluminescentes (LED) avec le logiciel WIEN2k en nous concentrant sur les propriétés optiques et électriques des éléments $I I-V I$.

- La deuxième partie traite de la conception, de la mise en ouvre et des essais de différents prototypes de communication VLC pour la transmission analogique et numérique en mode simplex et semi-duplex. Nous avons démontré qu'un système OWC utilisant une source à large bande (c'est-à-dire une LED) peut être utilisé non seulement pour la transmission de données, mais aussi pour la transmission d'énergie sans fil. De plus, le problème de synchronisation et la détection du niveau " 1 " ou "0" d'un bit se posent souvent dans les systèmes de communication optique sans fil. Ceci est dû à la nature de l'atténuation de la lumière sur la distance et au problème de l'alignement (NLOS) entre l'émetteur et le récepteur. Pour faire face à ce problème, un protocole de communication assurant une transmission fiable des données numériques avec un algorithme de détection adaptative du niveau des bits a été fourni et son efficacité a été démontrée par la transmission de textes et d'images.

- En outre, cette thèse apporte une solution pour la mise en œuvre d'émetteurs à multiplexage par répartition en longueur d'onde - multiplexage par répartition en fréquence orthogonale (WDM-OFDM) basée sur l'utilisation de sources à large bande dans le spectre infrarouge pour les réseaux de fibres bidirectionnels centralisés. Malgré la dispersion chromatique qui empêche l'utilisation de ce type de sources optiques, l'inclusion de certaines structures avant la détection permet la transmission de signaux OFDM dans les liaisons optiques. La réutilisation des porteuses, l'allocation dynamique de la largeur de bande et la transmission de signaux OFDM multi bande seront démontrées expérimentalement en utilisant des sources optiques à large bande dans les réseaux WDM.

Les principaux résultats obtenus au cours de ce travail de thèse démontrent les procédures d'étude, pour chaque partie, l'efficacité des solutions proposées ainsi que les contraintes rencontrées.

Mots clés : OWC, VLC, FSO, Fibre, Lumière, sources large bande, LED, Modulation optique, Emetteur, Récepteur. 
Las comunicaciones ópticas inalámbricas (OWC) constituyen una tecnología muy prometedora para el desarrollo de futuras comunicaciones inalámbricas. De hecho, ha despertado un interés creciente entre los investigadores y varias empresas de todo el mundo trabajan actualmente en el desarrollo de redes inalámbricas de muy alta velocidad. Las comunidades científica e industrial consideran la OWC como una tecnología complementaria en sus diversas formas: comunicaciones ópticas en el espacio libre (FSO), comunicaciones de luz visible (VLC) o fidelidad de la luz (Li-Fi). El espectro óptico ha sido considerado durante muchos años como una gran oportunidad para las comunicaciones inalámbricas, especialmente debido a la saturación del espectro de radiofrecuencia (RF).

Esta disertación trata del uso de fuentes de banda ancha en sistemas de transmisión de luz visible (VLC), así como en sistemas de transmisión en el espectro infrarrojo por fibra óptica. En el trabajo de investigación realizado se pueden distinguir tres partes:

- En la primera parte, se considera el estudio y la simulación de componentes de Diodos Emisores de Luz (LED) con el software WIEN2k centrándose en las propiedades ópticas y eléctricas de los elementos $I I-V I$.

- La segunda parte trata del diseño, la implementación y las pruebas de diferentes prototipos de comunicación VLC para la transmisión analógica y digital en modo simplex y semidúplex. Hemos demostrado un sistema OWC empleando una fuente de banda ancha (LED) para la transmisión no sólo de datos, sino también para la transmisión inalámbrica de energía. Además, se aborda el problema de la sincronización y la detección del nivel "1" o "0" de un bit en los sistemas de comunicación inalámbrica óptica implementados que surge como consecuencia de la atenuación de la luz a lo largo de la distancia y al problema de la pérdida de línea de visión (NLOS) entre el emisor y el receptor. Para hacer frente a este problema, se ha proporcionado un protocolo de comunicación que garantiza la transmisión fiable de datos digitales con un algoritmo de detección de nivel de bits adaptativo y se ha demostrado su eficacia mediante la transmisión de textos e imágenes.

- Además, esta tesis aporta una solución para la implementación de transmisores multiplexados en redes con división de longitud de onda (WDM) para formatos de modulación con multiplexación por división de frecuencia ortogonal (OFDM) basados en el uso de fuentes de banda ancha en el espectro infrarrojo para redes de fibra bidireccionales centralizadas. A pesar de las limitaciones impuestas por la dispersión cromática en el uso de este tipo de fuentes ópticas, la inclusión de ciertas estructuras antes de la detección permite la transmisión de señales OFDM en enlaces ópticos. En este trabajo se ha demostrado experimentalmente la reutilización de portadoras, la asignación dinámica de ancho de banda y la transmisión de señales OFDM multibanda mediante el uso de fuentes ópticas de banda ancha en redes WDM.

Los principales resultados obtenidos en cada parte de esta tesis doctoral muestran los procedimientos de estudio, la eficacia de las soluciones propuestas y las limitaciones encontradas.

Palabras clave: OWC, VLC, FSO, Fibra, Luz, Fuentes de banda ancha, LED, Modulación óptica, Emisor, Receptor 
Les comunicacions òptiques sense fils (OWC) constitueixen una tecnologia molt prometedora per al desenvolupament de futures comunicacions sense fils. De fet, ha despertat un interés creixent entre els investigadors i diverses empreses de tot el món treballen actualment en el desenvolupament de xarxes sense fils de molt alta velocitat. Les comunitats científica i industrial consideren la OWC com una tecnologia complementària en les seues diverses formes: comunicacions òptiques en l'espai lliure (FSO), comunicacions de llum visible (VLC) o fidelitat de la llum (Li-Fi).

L'espectre òptic ha sigut considerat durant molts anys com una gran oportunitat per a les comunicacions sense fils, especialment a causa de la saturació de l'espectre de radiofreqüència (RF).

Aquesta dissertació tracta de l'ús de fonts de banda ampla en sistemes de transmissió de llum visible (VLC), així com en sistemes de transmissió en l'espectre infraroig per fibra òptica. En el treball de recerca realitzat es poden distingir tres parts:

- En la primera part, es considera l'estudi i la simulació de components de Díodes Emissors de Llum (LED) amb el software WIEN2k centrant-se en les propietats òptiques i elèctriques dels elements II-VI.

- La segona part tracta del disseny, la implementació i les proves de diferents prototips de comunicació VLC per a la transmissió analògica i digital de manera simplex i semidúplex. Hem demostrat un sistema OWC emprant una font de banda ampla (LED) per a la transmissió no sols de dades, sinó també per a la transmissió sense fil d'energia. A més, s'aborda el problema de la sincronització i la detecció del nivell "1" o "0" d'un bit en els sistemes de comunicació sense fil òptica implementats, que sorgeix a conseqüència de l'atenuació de la llum al llarg de la distància i al problema de la pèrdua de línia de visió (NLOS) entre l'emissor i el receptor. Per a fer front a aquest problema, s'ha proporcionat un protocol de comunicació que garanteix la transmissió fiable de dades digitals amb un algorisme de detecció de nivell de bits adaptatiu i s'ha demostrat la seua eficàcia mitjançant la transmissió de textos i imatges.

- A més, aquesta tesi aporta una solució per a la implementació de transmissors multiplexats en xarxes amb divisió de longitud d'ona (WDM) per a formats de modulació amb multiplexació per divisió de freqüència ortogonal (OFDM) basats en l'ús de fonts de banda ampla en l'espectre infraroig per a xarxes de fibra bidireccionals centralitzades. Malgrat les limitacions imposades per la dispersió cromàtica en l'ús d'aquest tipus de fonts òptiques, la inclusió d'unes certes estructures abans de la detecció permet la transmissió de senyals OFDM en enllaços òptics. En aquest treball s'ha demostrat experimentalment la reutilització de portadores, l'assignació dinàmica d'amplada de banda i la transmissió de senyals OFDM multibanda mitjançant l'ús de fonts òptiques de banda ampla en xarxes WDM.

Els principals resultats obtinguts en cada part d'aquesta tesi doctoral mostren els procediments d'estudi, l'eficàcia de les solucions proposades i les limitacions trobades.

Paraules clau : OWC, VLC, FSO, Fibra, Luz, Fuentes de banda ancha, LED, Modulación óptica, Emisor, Receptor. 


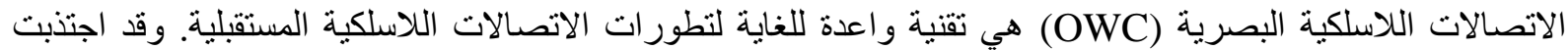

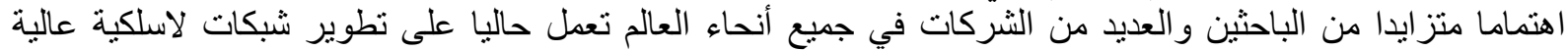

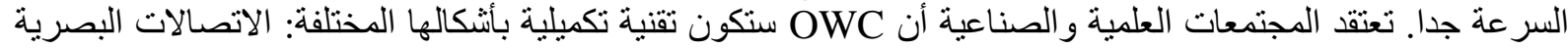

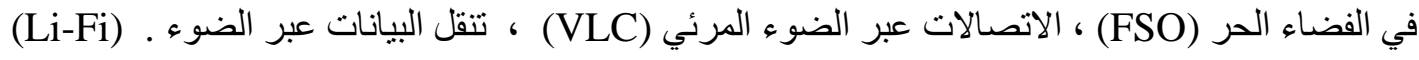
في الو اقع، نم اعتبار الطيف البصري لسنو ات عديدة كفرصة كبيرة للاتصالات اللاسلكية خاصة بسبب تثبع طيف التردد الر اديوي( RF (RF) . تتناول هذه الأطروحة استخدام مصادر كاملة النطاق في أنظمة نقل الضوء المرئي (vlc) و كذللك أنظمة الألياف. البصرية. لتنفيذ البحث، يمكن تمييز ثلاثة أجزاء:

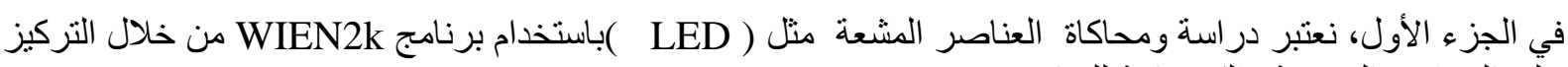

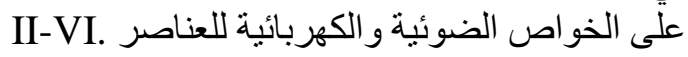

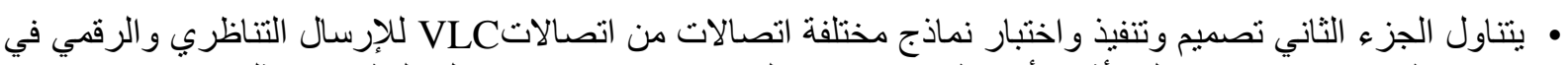

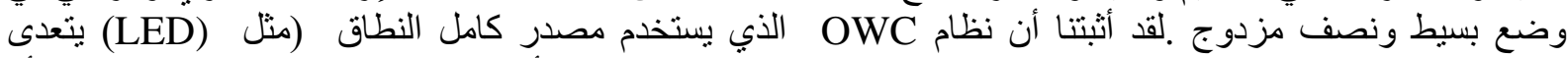

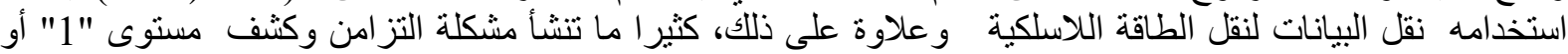

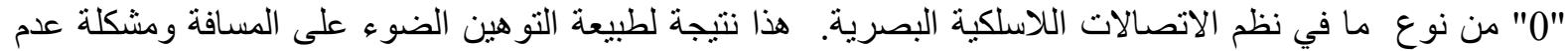

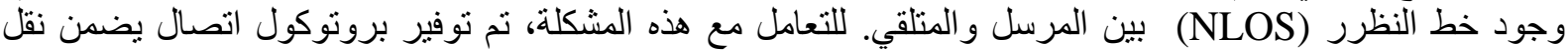
البيانات الرقمية الموثوقة مع خو ارزمية تكيفية للكثف عن مستوى وحدة الإرسال (Bit) وقد تم إنبات فعاليته من خلال نقل النصوص و الصور.

• بالإضافة إلى ذلك، توفر هذه الأطروحة حلا لتنفيذ محطات الإرسال ذات النطاقات المتعددة عن طريق التوزيع على التى

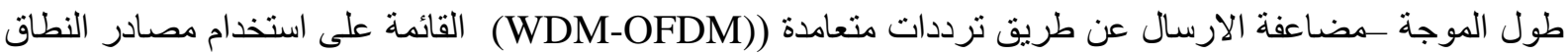

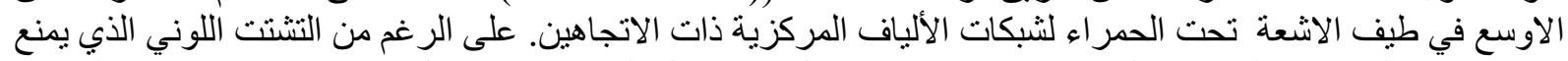

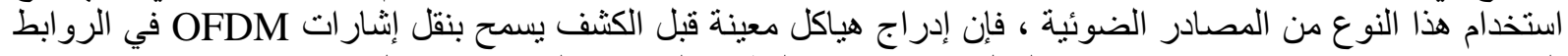

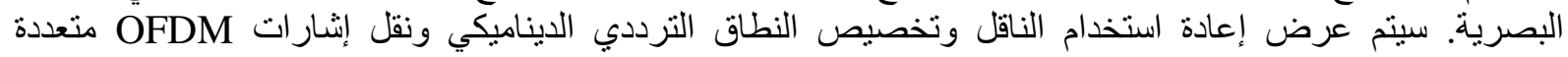

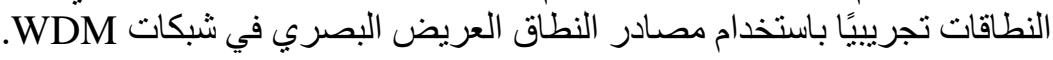

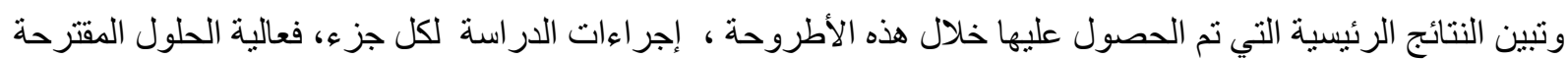
وكذللك الصعوبات التي واجنتها.

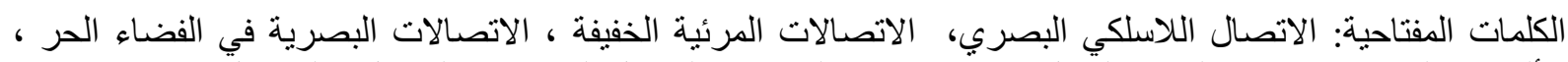

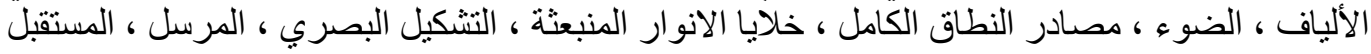

الكلمات المفتاحية : FSO ،VLC ، OWC ، الألياف ، الضوء ، مصادر النطاق العريض ، LED ، التعديل البصري ،

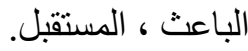




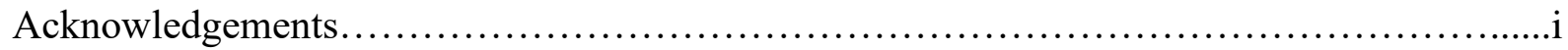

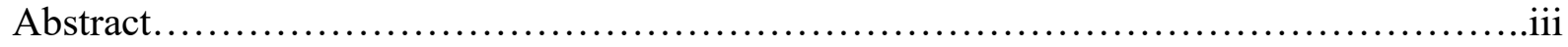

Table of contents................................................................ viii

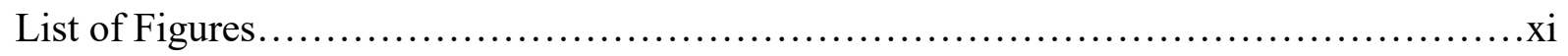

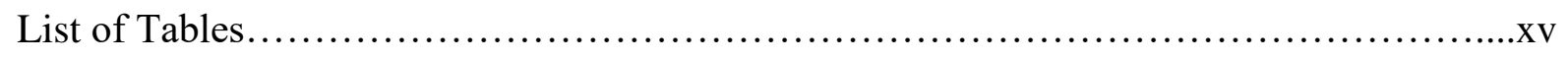

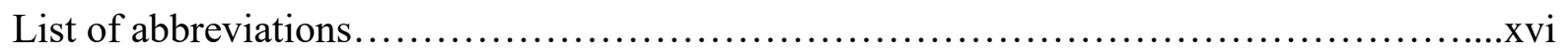

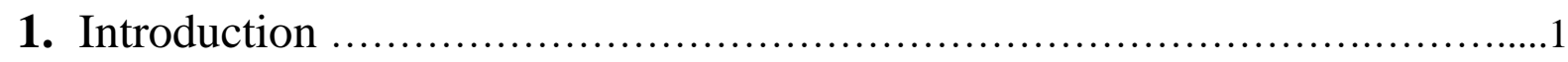

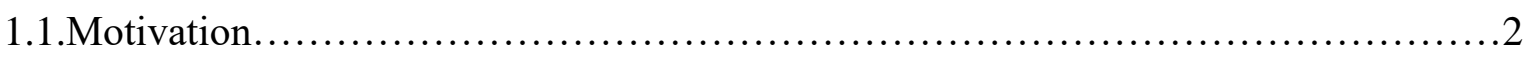

1.1.1. The current needs of telecommunications.......................................

1.1.2. Visible light communications......................................... 4

1.1.3. Broadband sources in fiber communications................................ 8

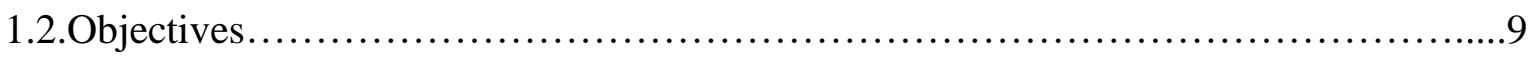

1.3. Organization of the thesis................................................. 10

2. Optoelectronic for optical wireless communications.........................12

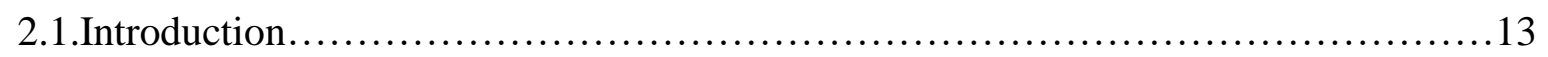

2.2.Optoelectronic properties of semiconductors................................. 13

2.2.1. Semiconductors ...................................................... 14

2.2.2. Semiconductor alloys............................................... 15

2.2.3. Properties of II-VI semiconductors.................................. 15

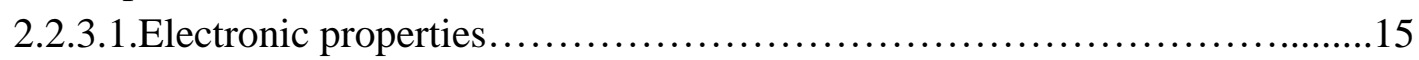

2.2.3.2.Optical properties............................................... 16

2.3.Design of Optoelectronics Materials .......................................... 17

2.3.1. WIEN2K software description...................................... 17

2.3.1.1.The Theory of the Functional Density....................................17

2.3.1.2.The Local Density Approximation (LDA) ............................... 18

2.3.1.3.The Generalized Gradient Approximation (GGA)........................ 19

2.3.2. The FP-LAPW method............................................ 19

2.3.3. The flowchart of WIEN2K...........................................20

2.4.Study of the $\mathrm{ZnSeO}$ material for optoelectronic devices in wireless communication...21

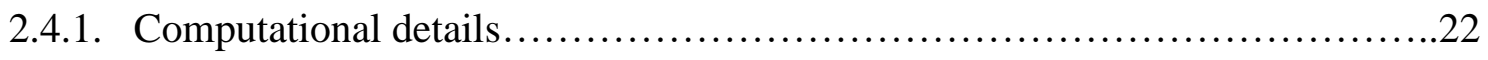

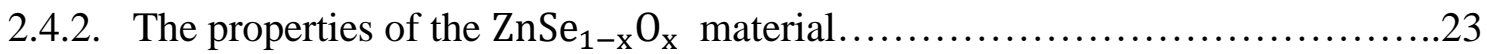

2.4.2.1.Physical properties............................................. 23

2.4.2.2.Electronic properties.......................................... 25

2.4.2.3. Optical properties............................................25

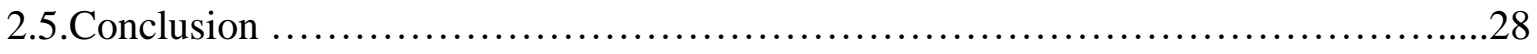

3. Optical Wireless Communications...........................................29

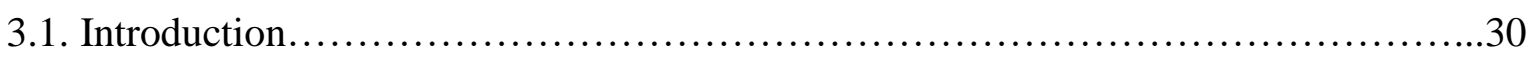

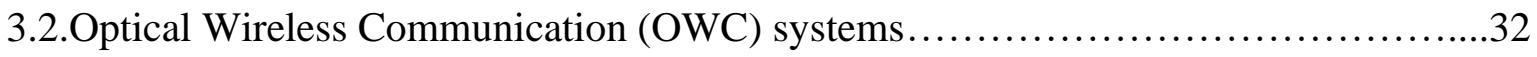

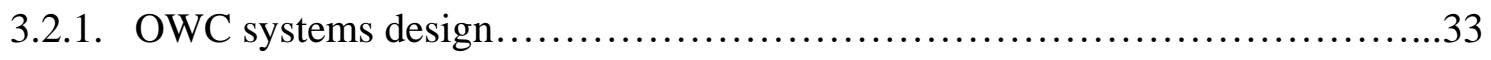

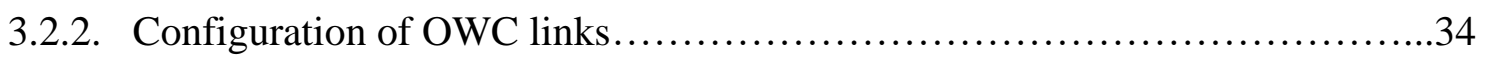




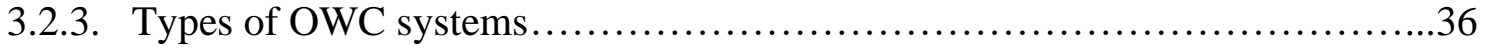

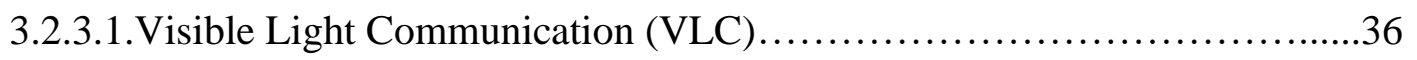

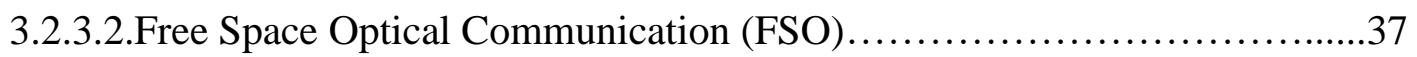

3.2.4. Optical modulation for OWC systems.................................. 38

3.2.4.1.Factors affecting modulation techniques for OWC systems................38

3.2.4.2.Types of modulation techniques used in OWC systems....................39

3.3. Design of Experimental prototypes.........................................43

3.3.1. Analog wireless transmission using visible light.........................43

3.3.2. System description................................................ 44

3.4. Experimentation and results..............................................45

3.4.1. Analog wireless transmission using visible light ......................... 45

3.4.2. Digital wireless transmission using visible light...........................50

3.4.2.1.Description of the transmitted data..................................50

3.4.2.2.Experimental results.........................................51

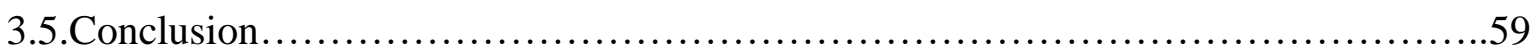

4. Optical OFDM signal transmission using a broadband source over SSMF...60

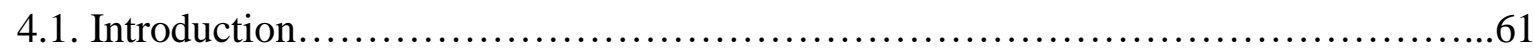

4.2. Broadband sources based optical networks ..................................62

4.2.1. Different broadband sources..........................................62

4.2.2. Fundamentals of signal transmission based on BBSs......................63

4.2.3. Tolerant dispersion systems for BBS based optical links...................66

4.3.Experimental setup..................................................... 70

4.3.1. Generation and reception of OFDM signal.............................. 70

4.3.1.1.OFDM signal parameters ........................................... 72

4.3.1.2.Symbol generation............................................... 73

4.3.1.3.Location and assignment of carriers................................ 73

4.3.1.4.Hermitian symmetry ............................................ 74

4.3.1.5.The cyclic prefix................................................ 74

4.3.1.6.Pre-emphasis filter............................................ 76

4.3.1.7.Signal clipping.................................................. 76

4.3.1.8.Data rate of transmitted signal.......................................77

4.3.1.9. Reception of the OFDM signal......................................78

4.3.1.10. Quality of the OFDM signal..................................... 79

4.3.2. Optical components................................................ 81

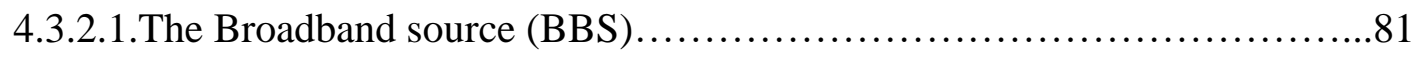

4.3.2.2.Multiport Tunable Optical Filter (MTOF) ................................ 81

4.3.2.3.Electro-Optic Modulator (EOM) ................................. 82

4.3.2.4.Polarization controller.............................................. 82

4.3.2.5.Receiver.................................................... 82

4.3.3. OOFDM-WDM experimental network............................... 83

4.4.Experimental results and applications.................................... 86

4.4.1. Carrier reuse for bidirectional transmission............................ 86 
4.4.2. Dynamic bandwidth allocation.......................................... 88

4.4.3. Multiple users in reconfigurable networks............................. 90

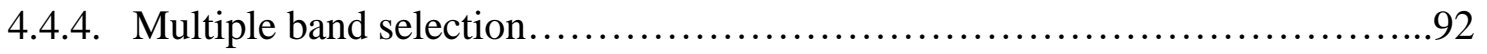

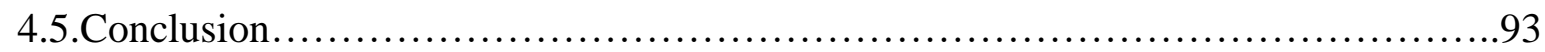

5. Conclusions and future prospects....................................94

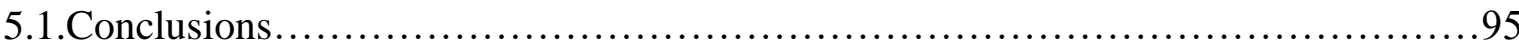

5.1.1. Optoelectronic devices for optical telecommunications.....................95

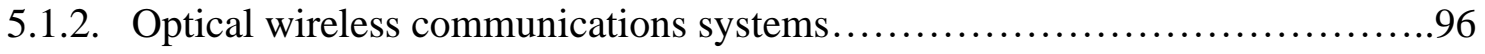

5.1.3. Bidirectional WDM-OOFDM access networks based on broadband sources...97

5.2.Future research lines...................................................97

Bibliography

Appendixes 
Fig. 1.1: Percentage of individuals using the internet, by region and development status [ITU 2019]. CIS: The Commonwealth of Independent States, LDCs: The least developed countries. .2

Fig. 1.2: Global visual network Index predictions [Cis 2018]...........................

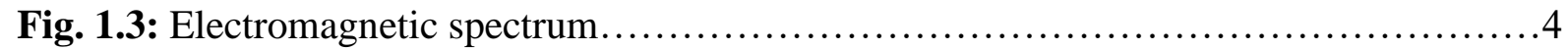

Fig. 1.4: DOW configuration.......................................................

Fig. 1.5: Principle of spectral slicing technique [Gra 2013]..........................

Fig. 2.1: Interband transitions for semiconductors. (a) Direct bandgap, (b) Indirect

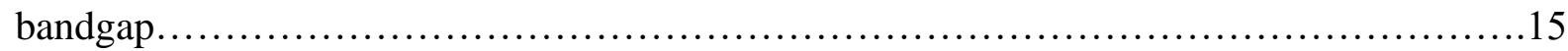

Fig. 2.2: Band gap energy according to the mesh parameter for some semiconductors.......16

Fig. 2.3: Interaction of radiation and electrons in semiconductors.......................17

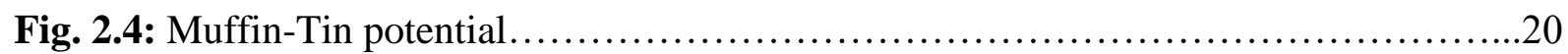

Fig. 2.5: The Zinc Sphalerite structure of a II-VI binary semiconductor $(\mathrm{ZnSe}) \ldots \ldots \ldots \ldots . .22$

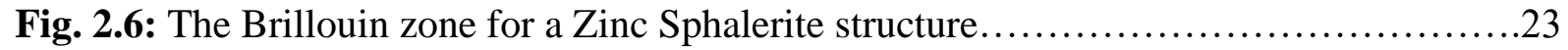

Fig. 2.7: Lattice constant versus oxygen concentration in $\mathrm{ZnSe}_{1-x} O_{x}$. According to Fig. 2.7, The lattice constants found using LDA approximation for binary $Z n S e$ and $Z n O$ are $5.5652 \AA$ and $4.48 \AA$, respectively. However, using the GGA approximation, we have found $5.7270 \AA$

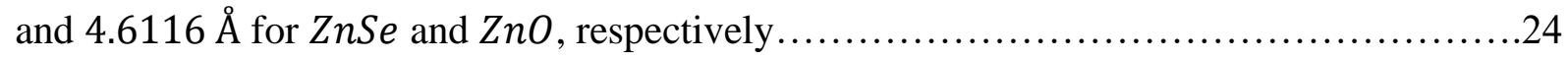

Fig. 2.8: Bulk modulus versus oxygen concentration in $Z n S e_{1-x} O_{x} \ldots \ldots \ldots \ldots \ldots \ldots \ldots \ldots . . . \ldots \ldots$

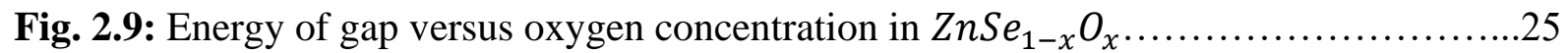

Fig. 2.10: The real and imaginary parts of the dielectric function of $Z n S e_{1-x} O_{x}$ compound to the structure Zinc Sphalerite based on LDA and GGA approximations: (a) $\mathrm{ZnSe}$, (b) $\mathrm{ZnO}$, (c)

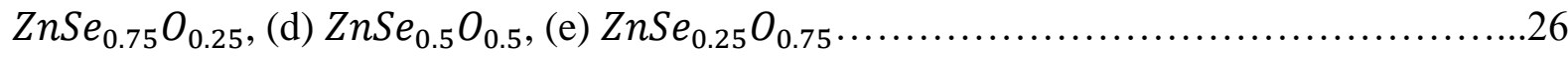

Fig. 2.11: The real $(n)$ and imaginary parts $(k)$ of the index of refraction of the ternary compound $\mathrm{ZnSe}_{1-x} \mathrm{O}_{x}$ for the structures zinc sphalerite based on LDA and GGA approximation. (a) $\mathrm{ZnSe}$, (b) $\mathrm{ZnO}$, (c) $\mathrm{ZnSe}_{0.75} \mathrm{O}_{0.25}$, (d) $\mathrm{ZnSe}_{0.5} \mathrm{O}_{0.5}$, (e) $\mathrm{ZnSe}_{0.25} \mathrm{O}_{0.75} \ldots .27$

Fig. 2.12: Absorption coefficient according to the energy $Z n S e_{1-x} O_{x}$ for the structure zinc sphalerite with LDA and GGA. (a) $Z n S e$, (b) $Z n O$, (c) $Z n S e_{0.75} O_{0.25}$, (d) $Z n S e_{0.5} O_{0.5}$, (e)

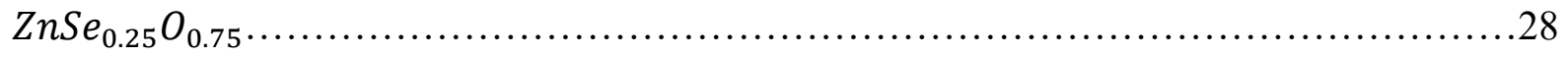

Fig. 3.1: Examples of OWC for different ranges of communications [Cho 2018].............33

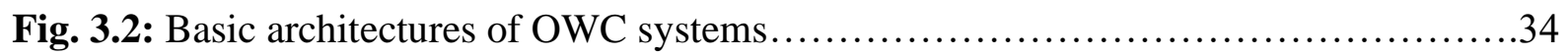

Fig. 3.3: Classification of Indoor OWC communication links [Kah 1997].................35

Fig. 3.4: Part of the electromagnetic (EM) spectrum showing the frequency and wavelength ranges for each band........................................................... 36 
Fig. 3.5: Basic block diagram of an FSO system [Kha 2014] ..............................

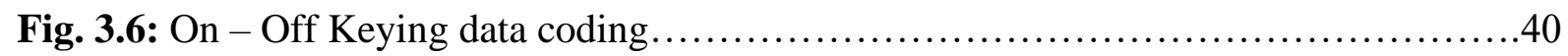

Fig. 3.7: 4-PAM Modulation................................................ 41

Fig. 3.8: Schematic diagram showing the difference between Pulse Width Modulation (PWM), Pulse Position Modulation (PPM), Variable Pulse Position Modulation (VPPM) ...42

Fig. 3.9: The frequency spectrum of our transmitted audio signal.......................43

Fig. 3.10: Time presentation of the audio signal...................................44

Fig. 3.11: Block diagram of simultaneous Optical Wireless audio transmission and energy

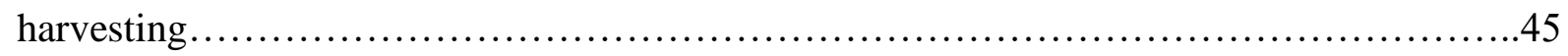

Fig. 3.12: The audio signal to be transmitted. Time is represented in the $x$-axis.............46

Fig. 3.13: TDA7297 15w Dual Channel AC/DC $12 \mathrm{~V}$-Digital audio amplifier..............47

Fig. 3.14: Audio signal amplification and Dc bias addition ............................47

Fig. 3.15: The received Audio signals with a separation distance between emitter (Led) and receiver (photovoltaic cell) of: (a) $30 \mathrm{~cm}$, (b) $1.05 \mathrm{~m}$ and (c) $2.30 \mathrm{~m} \ldots \ldots \ldots \ldots \ldots \ldots \ldots . \ldots . \ldots . \ldots . \ldots$

Fig. 3.16: The photovoltaic cell (JZ-PV11570) and the mini Solar Lipo charger (CN3065).

Fig. 3.17: The illuminance of the photovoltaic cell vs the distance between transmitter and receiver.

Fig. 3.18: The power of the audio signal received at the loudspeakers vs the distance between the transmitter and the receiver.

Fig. 3.19: Synoptic diagram of an indoor VLC link in simplex mode. .51

Fig. 3.20: Bits detection problem when receiving a succession of identical bits.............52

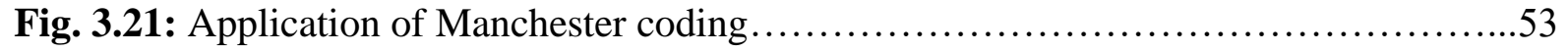

Fig. 3.22: Frame organization in the proposed protocol of communication.................54

Fig. 3.23: Proposed Protocol of transmission flowchart...............................54

Fig. 3.24: Implementation of our text transmission system via visible light...............55

Fig. 3.25: Example of transmission and reception of a text via visible light. (a) IDE interface of the PC for transmission, (b) IDE interface of the PC reception..........................55

Fig. 3.26: Transmission of an RGB image of size $(50 \times 50)$ using visible light in free space.

Fig. 3.27: Oscilloscope visualization of the emitted signal after Manchester encoding and the received signal from the photodiode. .58

Fig. 3.28: Image reconstitution on the computer screen ................................58

Fig. 4.1: Schematic of a transmission system using an optical broadband source. .64 
Fig. 4.2: Transfer functions using a broadband source with different values of $\left(\delta \omega_{3 d B}\right) / 2 \pi$ [GHz]: 0.125 (blue), 12.5 (Orange), 50 (yellow), 100 (violet), 500 (green) in cases (a) Rectangular distribution and (b) Gaussian distribution...............................65

Fig. 4.3: Transmission structure based on a Mach-Zehnder interferometer and a VDL......66

Fig. 4.4: Scheme of transmission with Broadband Sources based on Mach-Zehnder interferometer. .66

Fig. 4.5: (a) Optical spectrum of the broadband source, (b) optical spectrum at the output of the $6 \mathrm{~nm}$ bandwidth optical filter.

Fig. 4.6: (a) Optical spectrum at the output of the MZI-VDL. (b) Electrical transfer function of the proposed system.

Fig. 4.7: The response of the transfer function for different $\Delta \tau$ (blue: $6.38 p s$, red: $3.83 p s$, yellow: $19.13 \mathrm{ps}$, grey: $38.26 \mathrm{ps}$ and green: $57.40 \mathrm{ps}$ ).

Fig. 4.8: OFDM-WDM network using broadband source. BBS: Broadband source (Optical). EOM: Electro-Optical Modulator. AWG: Arbitrary Waveform Generators. MZI-VDL: MachZehnder Interferometer with Variable Delay Line. RTDO: Real Time Digital

Oscilloscope.

Fig. 4.9: (a) Experimental system for the transmission of OFDM signals in the electrical link, (b) Block diagram of generation and reception of an OFDM symbol. .71

Fig. 4.10: Two pilot location patterns: (a) comb-type, (b) block-type....................73

Fig. 4.11: An example of the insertion of a cyclic $25 \%$ prefix on a 128 carriers signal: (a) original signal, (b) signal with cyclic prefix...................................... 75

Fig. 4.12: Application of the pre-emphasis filter................................... 76

Fig. 4.13: Application of clipping of the temporal signal.............................77

Fig. 4.14: Centralized bidirectional OOFDM-WDM network using broadband source. BBS: BroadBand Source (Optical), MTOF: Multiport Tunable Optical Filter, EOM: Electro-Optical Modulator, MUX: Multiplexer, DEMUX: Demultiplexer, PS: Passive splitter, TMZI: Tunable Mach-Zehnder Interferometers, DL: Downlink, UL: Uplink, OF: Optical Fiber, PD:

Photodetector, AWG: Arbitrary Waveform Generator (RF), RTDO: Real Time Digital Oscilloscope, CO: Central Office, ONU: Optical Network Unit..........................83

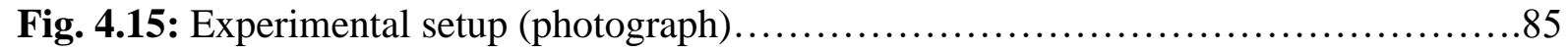

Fig. 4.16: Electrical transfer function of the proposed system. (a) Downlink, (b) Uplink.....86

Fig. 4.17: Power spectral density of received electrical signal: (a) Downstream, (b) Upstream.

Fig. 4.18: Downstream transmission: $750 \mathrm{MHz}$ 16-QAM OFDM signal carried at $5 \mathrm{GHz}$. EB2B results: (a) Constellation, (c) EVM per subcarrier, (e) EVM per symbol. After $10 \mathrm{~km}$ fiber transmission link: (b) Constellation, (d) EVM per subcarrier, (f) EVM per symbol......87

Fig. 4.19: Upstream transmission: $750 \mathrm{MHz}$ QPSK OFDM signal carried at 3.5GHz. EB2B 
results: (a) Constellation, (c) EVM per subcarrier, (e) EVM per symbol. After $10 \mathrm{~km}$ fiber transmission uplink: (b) Constellation, (d) EVM per subcarrier, (f) EVM per symbol........88

Fig. 4.20: (a) EB2B power spectral density for $B W=2.66 \mathrm{GHz}$ (inset: constellation), (b) EVM per subcarrier, (c) EVM per symbol............................................. 88

Fig. 4.21: Transmission system response when optical carrier bandwidth is 3.9nm: (a) Electrical transfer function, (b) Power spectral density of recovered electrical signal when modulating signal is the one depicted in Fig. 4.19.

Fig. 4.22: Transmission system response when optical carrier bandwidth is $8 \mathrm{~nm}$ (modulating signal is depicted in Fig. 4.20): (a) Electrical transfer function, (b) Power spectral density of recovered electrical signal..................................................... 89

Fig. 4.23: The schematic for reconfigurable multi-users networks.......................90

Fig. 4.24: The spectral bands assigned to four ONUs in the WDM network................91

Fig. 4.25: shows the electrical frequency transfer functions measured at each ONU where the TMZIs have been configured with differential delays of 1.76, 3.14, 6.28 and $13.32 \mathrm{ps,}$ respectively. The corresponding insets show the recovered power spectral density of the recovered signal and demonstrate effective transmission.

Fig. 4.26: Electrical RF transfer function at: (a) ONU 1, (b) ONU 2, (c) ONU 3 and (d) ONU

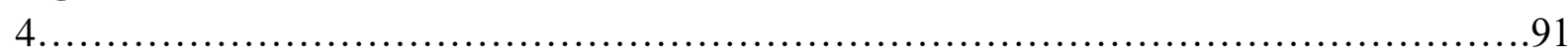

Fig. 4.27: Experimental system with two TMZIs in cascade. .92

Fig. 4.28: Electrical RF transfer function: (a) TMZIs set at 2.5 and $5 \mathrm{GHz}$, (b) TMZIs set at 3 and $5 \mathrm{GHz}$, (c) TMZIs set at 3.5 and $6 \mathrm{GHz}$.... 
Table 1.1: Approximated spectral colors of visible light...............................4

Table 1.2: Comparison between VLC and RF............................................

Table 2.1: Commercial applications for optoelectronic devices [Abde, 2006]..............14

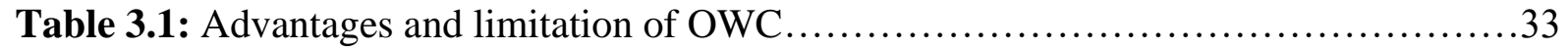

Table. 3.2: COMPARISON of different OWC systems..................................

Table 3.3: Specifications of the equipment in the Analog wireless transmission using visible

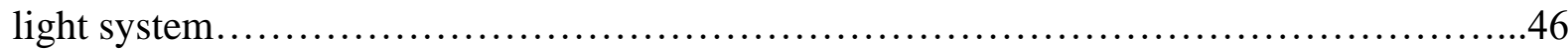

Table 3.4: Performance of the text transmission system via visible light in detected erros...56

Table 4.1: Typical linewidth of different optical sources [Chi 2018]. DFB: Distributed FeedBack, DBR: Distributed Bragg Reflector, FP: Fabry-Perot, ECL: External Cavity Laser, VCSL: Vertical-Cavity Surface-Emitting Laser, MWQ: Multiple Quantum Well, sLED: superluminescent Light-Emitting Diode, LED: Light-Emitting Diode, ASE: Amplified Spontaneous Emission.

Table 4.2: Optical source with different width, $\delta \omega_{3 d B} / 2 \pi$, values used in the theoretical model and corresponding FWHM widths of the electrical transfer function for rectangular and Gaussian sources

Table 4.3: Organization of the data at the input of the IFFT to respond to Hermitian

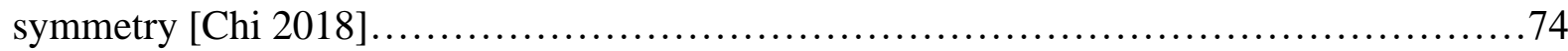

Table. 4.4: The characteristics of BBS NP Photonics C\&L Band ASE Source...............81

Table 4.5: The optical characteristics of MTOF (Finisar WaveShaper $4000 S$ ).............81

Table 4.6: The characteristics of EOM Avanex.......................................... 82

Table 4.7: The specifications of the VDL General Photonics.........................82

Table 4.8: The specifications of the balanced photodetector $u 2 t$ photonics BPDV2020R....83

Table 4.9: Specifications of the equipment in the OOFDM-WDM proposed network. .85 
ACO-OFDM

ADC

AlGaAs

ASE

APD

AWG

BBS

BER

BLE

BLS

BW

CCDF

CCI

CdTe

CO

CO-OFDM

CP

CSK

CWDM

DAC

DBR

DC

DCF

DCO-OFDM

DD

DD-OOFDM

DEMUX

DFB

DFT

DL

DM

DML

DMT

DOW

DPO

DSB

EAM

EB2B

ECL
Asymmetrically Clipped Optical OFDM

Analog to Digital Converter

Aluminium Gallium Arsenide

Amplified Spontaneous Emission

Avalanche diode

Arbitrary Waveform Generators

BroadBand source

Bit Error Rate

Bluetooth Low Energy

Broadband Light Source

Bandwidth

Complementary Cumulative Distribution Function

Co-Channel Interference

Cadmium Telluride

Central Office

Coherent Detection OFDM

Cyclic Prefix

Color-Shift Keying

Coarse Wavelength Division Multiplexing

Digital to Analog Converter

Distributed Bragg Reflector

Direct Current

Dispersion Compensating Fiber

DC biased Optical OFDM

Direct Detection

Direct-Detection Optical OFDM

Demultiplexer

Distributed Feedback

Density Functional Theory

Downlink

Dispersion-Managed

Directly Modulated Laser

Discrete Multi-tone Modulations

Diffuse Optical Wireless

Digital Phosphor Oscilloscope

Double Side-band

Electro Absorption Modulator

Electrical Back to Back

External Cavity Laser 


\begin{tabular}{|c|c|}
\hline EDFA & Erbium Doped Fiber Amplifier \\
\hline EM & Electromagnetic \\
\hline EOM & Electro-Optical Modulator \\
\hline EON & Elastic Optical Networks \\
\hline EVM & Error Vector Magnitude \\
\hline FBG & Fiber Bragg grating \\
\hline FDD & Frequency Division Duplexing \\
\hline FEC & Forward Error Correction \\
\hline FFT & Fast Fourier Transform \\
\hline FOV & Field of View \\
\hline $\mathbf{F P}$ & Fabry-Perot \\
\hline FP-LAPW & Full Potential Linearized Augmented Plane Waves \\
\hline FPGA & Field-Programmable Gate Array \\
\hline FSO & Free Space Optical \\
\hline FWHM & Full Width at Half Maximum \\
\hline GaAs & Gallium Arsenide \\
\hline GaN & Nitride of Gallium \\
\hline Ge & Germanium \\
\hline GGA & Generalized Gradient Approximation \\
\hline GHz & Giga-Hertz \\
\hline GPS & Global Positioning System \\
\hline GVD & Group Velocity Dispersion \\
\hline HHI & Heinrich Hertz Institute \\
\hline HOM & Higher-Order Mode \\
\hline IFFT & Inverse FFT \\
\hline IM/DD & Intensity Modulation/ Direct Detection \\
\hline InGaAs & Indium Gallium Arsenide \\
\hline InGaP & Indium Gallium Phosphide \\
\hline InP & Indium Phosphide \\
\hline IPS & Indoor Positioning Systems \\
\hline IR & Infrared \\
\hline IrDA & Infrared Data Association \\
\hline ISM & Industrial Scientific and Medical \\
\hline ISI & Inter Symbol Interference \\
\hline ITS & Intelligent Transportation System \\
\hline LAN & Local-Area Network \\
\hline LD & Laser Diode \\
\hline LDA & Local Density Approximation \\
\hline LDC & Last Developed Countries \\
\hline
\end{tabular}




\begin{tabular}{|c|c|}
\hline LED & Light Emitting Diode \\
\hline Li-Fi & Light Fidelity \\
\hline Lo & local orbitals \\
\hline LOS & Line-of-Sight \\
\hline MCM & Multi-Carrier Modulation \\
\hline MHz & Mega-Hertz \\
\hline MMF & Multimode Fiber \\
\hline MMI & Multi Mode Interference \\
\hline MLL & Mode-Locked Laser \\
\hline MT & Muffin tin \\
\hline MTOF & Multiport Tunable Optical Filter \\
\hline MUX & Multiplexer \\
\hline MWQ & Multiple Quantum Well \\
\hline MZI & Mach-Zehnder Interferometer \\
\hline MZM & Mach-Zehnder Modulator \\
\hline NLOS & Non Line-of-Sight \\
\hline NRZ & Non Return to Zero \\
\hline OF & Optical Fiber \\
\hline OFDM & Orthogonal Frequency Division Multiplexing \\
\hline OFDMA & OFDM Access \\
\hline $\mathbf{O L}$ & Local Oscillator \\
\hline OLT & Optical Line Terminals \\
\hline ONU & Optical Network Unit \\
\hline OOFDM & Optical OFDM \\
\hline OOK & On-Off-Keying \\
\hline OWC & Optical Wireless Communication \\
\hline PA & Power Amplifier \\
\hline PAM & Pulse Amplitude Modulation \\
\hline PAPR & Peak-to-Average Power Ratio \\
\hline PD & Photodetector \\
\hline PDA & Personal Digital Assistant \\
\hline PIN & Junction p-i-n \\
\hline PLC & Power Line Communication \\
\hline PMD & Polarization Mode Dispersion \\
\hline PON & Passive Optical Network \\
\hline PPM & Pulse Position Modulation \\
\hline PRL & Photonics Research Labs \\
\hline PS & Passive Splitter \\
\hline PSK & Phase-Shift keying \\
\hline PWM & Pulse Width Modulation \\
\hline
\end{tabular}




\begin{tabular}{|c|c|c|}
\hline QAM & Quadrature Amplitude Modulation & \\
\hline QPSK & Quadrature Phase-Shift keying & \\
\hline RF & Radio Frequency & \\
\hline RGB & Red Green Blue & \\
\hline RoF & Radio over Fiber & \\
\hline RSOA & Reflective Semiconductor Optical Amplifiers & \\
\hline RTDO & Real Time Digital Oscilloscope & \\
\hline $\mathbf{R Z}$ & Return-to-Zero & \\
\hline SCF & Self Consistent Field & \\
\hline SC-FDE & Single-Carrier Frequency-Domain Equalization & \\
\hline Si & Silicon & \\
\hline sLED & superluminescent LEDs & \\
\hline SMF & Single Mode Fiber & \\
\hline SNR & Signal to Noise Ratio & \\
\hline SOA & Semiconductor Optical Amplifier & \\
\hline SSB & Single Side-Band & \\
\hline SSMF & Standard Single Mode Fiber & \\
\hline SS-WDM-PON & $\begin{array}{l}\text { Spectrum Sliced Wavelength-Division-Multiplexed Passive } \\
\text { Network }\end{array}$ & Optical \\
\hline TDD & Time Division Duplexing & \\
\hline TDM & Time Division Multiplexing & \\
\hline TMZI & Tunable Mach-Zehnder interferometer & \\
\hline TOD & Third-Order Dispersion & \\
\hline $\mathbf{U L}$ & Uplink & \\
\hline $\mathbf{U V}$ & Ultraviolet & \\
\hline V2I & Vehicle to Infrastructure & \\
\hline V2V & Vehicle to Vehicle & \\
\hline VCSEL & Vertical-Cavity Surface-Emitting Laser & \\
\hline VDL & Variable Delay Line & \\
\hline $\mathbf{V L}$ & Visible Light & \\
\hline VLC & Visible Light Communication & \\
\hline VLCC & Visible Light Communication Consortium & \\
\hline VPPM & Variable PPM & \\
\hline WDD & Wavelength Division Duplexing & \\
\hline WDM & Wavelength Division Multiplexing & \\
\hline WDM-PON & Wavelength-Division-Multiplexed Passive Optical Network & \\
\hline Wi-Fi & Wireless-Fidelity & \\
\hline
\end{tabular}


WLAN

$\mathrm{ZnO}$

ZnSe

$\mathrm{ZnS}$
Wireless Local Area Network

Oxyde de zinc

Zinc Selenide

Zinc Sulfide 
Chapter 1

\section{Introduction}




\subsection{Motivation}

\subsubsection{The current needs of telecommunications}

In the past decade, the world has seen a growing increase in the traffic carried by the telecommunication networks including the wireless networks. At the same time, manufacturers are installing wireless communication capabilities in stationary electronic devices and appliances to enable consumers to communicate with them remotely. More and more users enjoy the convenience of employing wireless mobile devices anywhere, both indoors and outdoors. However, the ever-increasing demand for broadband internet services has underpinned the need for further innovation, research and development in the new emerging communication technologies capable of delivering ultra-high data rates. According to [ITU 2019], internet use in developed countries has almost reached its maximum possible level in 2019 , with almost $87 \%$ of the population online, where Europe is the region where Internet use is the highest (82.5\%) and Africa is the lowest (28.2\%), as shown in Fig.1.1.

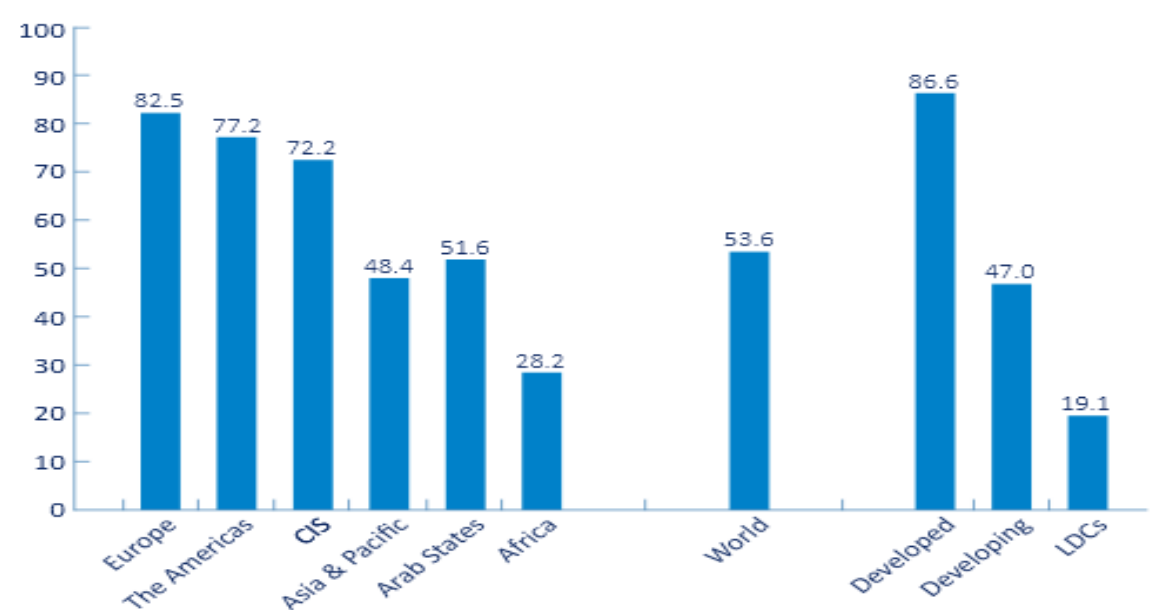

Fig. 1.1: Percentage of individuals using the internet, by region and development status [ITU 2019]. CIS: The

Commonwealth of Independent States, LDCs: The least developed countries.

Hundreds of millions of people send and receive messages and files via laptops, Personal Digital Assistants (PDAs) and other digital devices using Wi-Fi (Wireless-Fidelity) or cellular networks, the radio-frequency (RF) based wireless access technology. The CISCO company conducts an annual study that assesses the prospects for network data processing in the coming years. Its report for 2018 [Cis 2018] indicates an exponential trend in the growth of traffic generated by the Internet, as shown by the global network traffic statistics illustrated in Figure 1.2, as 254 and 396 exabytes per month in 2020 and 2022 respectively. 


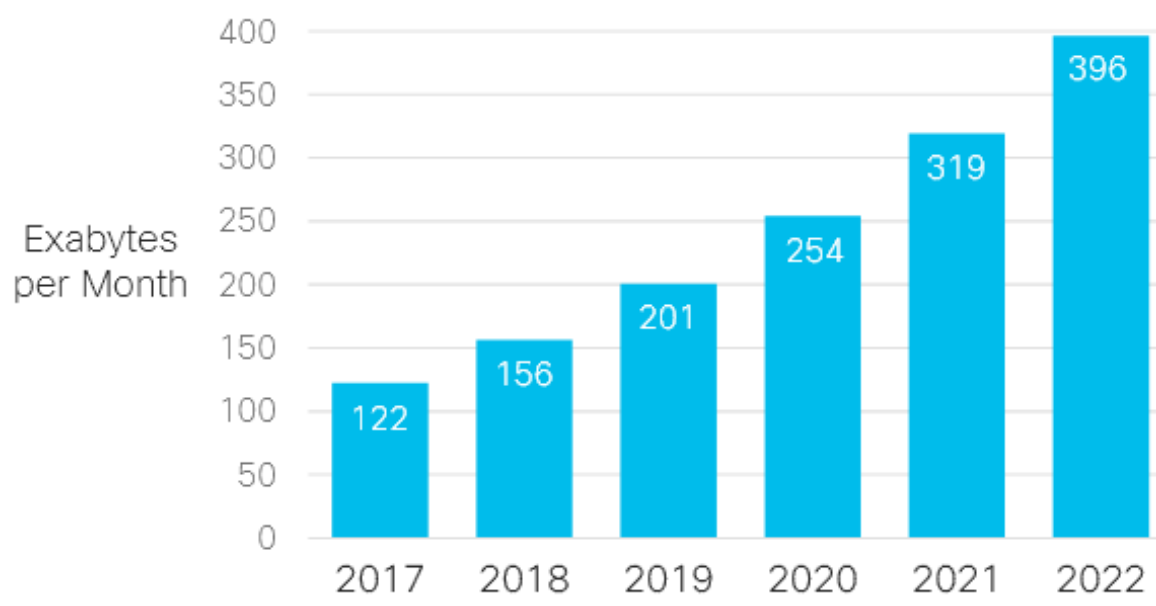

Fig. 1.2: Global visual network Index predictions [Cis 2018].

It can be observed that the global network traffic is continuously increasing at an expeditious pace and this forecasted global network traffic inflation is predicted to prevail in the successive years.

The different methods of data transmission are at the heart of the study subjects, in order to meet consumer demand. Wi-Fi is a reliable technology that has established itself worldwide, but its use is still controversial due to the unknown effects of radio waves on the human body. According to researchers, the use of the visible light spectrum has recently received particular attention as a technique to reduce the spectral load on radio systems.

Optical wireless technologies are one of the greatest success stories in the history of technological development, realizing the dream of humans to communicate from anywhere at any time. Optical Wireless communication (OWC) is a general term which refers to all types of optical communications like Visible Light Communication (VLC), free space optics (FSO), light fidelity ( $\mathrm{Li}-\mathrm{Fi})$ and infrared. The use of visible light for communications is driven by recent developments in optoelectronic components, especially optoelectronic components based on semiconductors. These have remarkable properties such as compactness, high electrical-to-optical conversion efficiency, low excitation voltage, thus greatly facilitating operating conditions, and reliability. The light-emitting diodes (LEDs) are paving the way for their full adoption as an alternative to incandescent and fluorescent lighting [Ste 2002]. The LEDs have been widely employed as lighting sources because of their long-life expectancy, low power consumption, and low cost. Meanwhile the signal to be transmitted can be modulated onto the LEDs without causing flicker perceived by the human eyes and detected by a photoelectric device. However, the $3 d B$ bandwidths of commercial LEDs are usually several $\mathrm{MHz}$, which restricts the data rate improvement.

Visible light communication based on LEDs has attracted more and more attention in recent years as it is considered to be a potential candidate wireless communication technique in the future [Mat 2019]. It can also be employed in environments which are sensitive to RF waves, for example, in airplanes, hospitals, etc., as it doesn't interfere with other modes of communication [Kom 2004]. VLC is more secure against hacking, as transmitted light is confined within the system's coverage area. Additionally, it offers high data rate compared to the conventional RF-based wireless technologies such as Wi-Fi and Bluetooth, thanks to its 
huge bandwidth [Pis 2012]. Most of the recent studies on VLC consider the LEDs as transmitter, such as [Erg 2015, Gao 2019, Li 2019a, Sin 2019, Zho 2019, Yeh 2019]. However, according to [Erg 2015], there are two main challenges in utilization of LEDs as transmitter, the limited bandwidth (Bandwidth can be extended with complex type of LEDs); and the nonlinearity (nonlinear relationship between current and optical power in LEDs becomes challenging in high data rate applications).

\subsubsection{Visible light communications}

The visible light spectrum is the segment of the electromagnetic spectrum that the human eye can view. More simply, this range of wavelengths is called visible light (Fig.1.3). Typically, the human eye can detect wavelengths from 380 to $700 \mathrm{~nm}$, i.e. 430 to $770 \mathrm{THz}$ in terms of frequency.

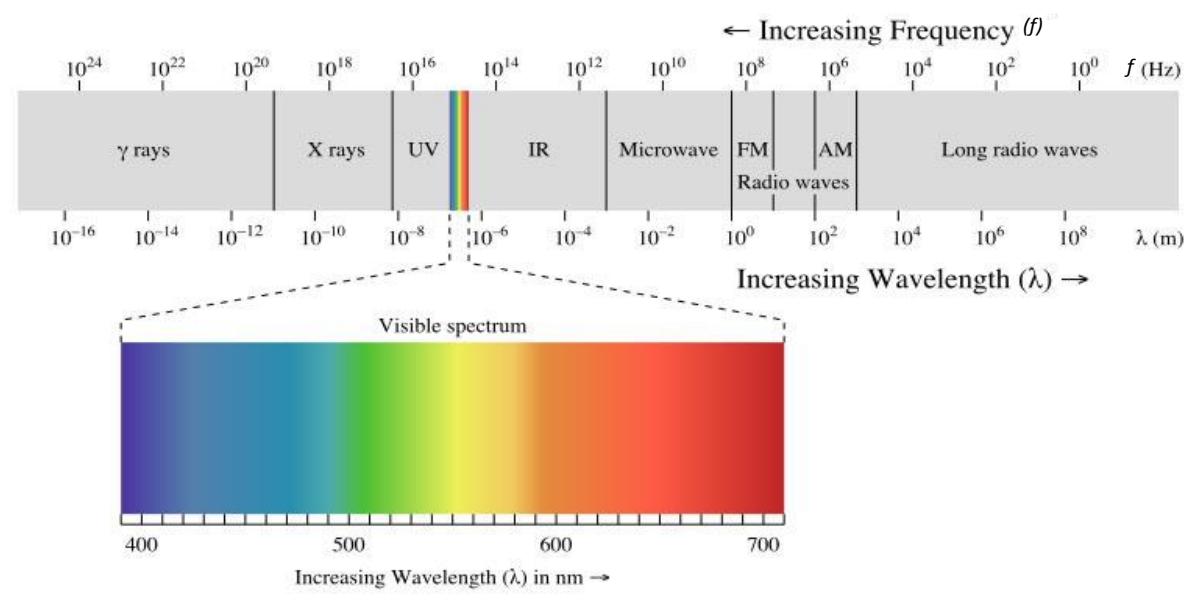

Fig. 1.3: Electromagnetic spectrum.

Several colors are generated by visible light in different, narrow frequency bands, called pure spectral colors. Table 1.1 shows the approximate spectral colors of visible light.

Table 1.1: Approximated spectral colors of visible light.

\begin{tabular}{|l|c|c|}
\hline \multicolumn{1}{|c|}{ Color } & Wavelength & Frequency \\
\hline Violet & $380-450 \mathrm{~nm}$ & $668-789 \mathrm{THz}$ \\
\hline Blue & $450-495 \mathrm{~nm}$ & $606-668 \mathrm{THz}$ \\
\hline Green & $495-570 \mathrm{~nm}$ & $526-606 \mathrm{THz}$ \\
\hline Yellow & $570-590 \mathrm{~nm}$ & $508-526 \mathrm{THz}$ \\
\hline Orange & $590-620 \mathrm{~nm}$ & $484-508 \mathrm{THz}$ \\
\hline Red & $620-750 \mathrm{~nm}$ & $400-484 \mathrm{THz}$ \\
\hline
\end{tabular}

OWC covers the entire light spectrum, whereas VLC employs the Visible Light spectrum. In OWC, the message can be transmitted by intensity-modulation and direct detection (IM-DD) method, where the data is modulated as the envelop of the electromagnetic radiation which is photo-detected at the receiver [Kah 1997, Han 2012]. IM-DD relies on the use of electrical-tooptical transceiver, such as light emitting diodes (LEDs) and laser diodes (LDs) at the 
transmitter, and optical-to-electrical transceivers like photodiodes (PDs) at the receiver. In recent years researchers focused on the use of alternative modulation techniques for VLC transmission. They have shown that Orthogonal frequency division multiplexing (OFDM) is also a promising technology for OWC. Other modulation techniques have been studied, for example in [God 2016], a study of a Flip-OFDM system was proposed using M-PSK and MQAM, and it was concluded that M-QAM has better performance than M-PSK in a FlipOFDM-modulated VLC system.

Visible light communication (VLC) refers to transmission of information by modulating a light source and reception by an optical receiver. A LED light source, on account of its fast switching and easy controllability, can be used as a VLC transmitter while providing flickfree illumination. Light Emitting Diodes (LEDs) has recently been expecting to replace traditional illumination sources due to more advantages compared to the existing incandescent lamps in terms of low power consumption long life expectancy and high tolerance to humidity. Typical LEDs have characteristics to light on/off very fast at ultra-high speed. When applying the visible light for the data information transmission, related problems in the field of wireless communications need to be resolved such as transmitting signal processing at ultra-high speed while harmless for human eye [Kom 2004], interference issues from other ambient light sources, short coverage range, atmospheric absorption, beam dispersion, and the most important thing is that the transmitter and receiver are in Line-of-Sight (LoS), hence non-LOS communication is difficult to be achieved. An indoor LED lighting based visible light communication systems is investigated as a convergence telecommunication technology which is integrated device used for both lighting and information transmission [Luo 2017]. Due to these advantages, the LEDs are not only ideal lighting sources for contributing to considerable energy savings, but also ideal transmitters for indoor localization and data communications, e.g. in [Zho 2019], a real-time data transmission of 10Mbps Ethernet over $120 \mathrm{~m}$ is realized under strong background light using a LED as transmitter.

Visible light communication (VLC) systems have become promising candidates to complement conventional radio frequency (RF) systems due to the increasingly saturated RF spectrum and the potentially high data rates that can be achieved by VLC systems. Over the last decade, significant research efforts have been directed towards the development of VLC systems due to their numerous advantages over RF communication systems, such as the availability of simple transmitters (light emitting diodes, (LEDs)) and receivers (silicon photo detectors), better security at the physical layer, improved energy efficiency due to the dual functionally (i.e., illumination and communication) and hundreds of $\mathrm{THz}$ of license-free bandwidth. However, several challenges must be faced by VLC systems to achieve high data rates (multi gigabits per second). These challenges include the low modulation bandwidth of the LEDs, inter symbol interference (ISI) due to multipath propagation, co-channel interference (CCI) and the light unit (i.e., VLC transmitter) should be "ON" all the time to ensure continuous communication.

However, the main challenge of the VLC system is that the modulation bandwidth of LEDs, and most commercial LEDs is limited to $2 \mathrm{MHz}$ for white LEDs, making Gbps communication links currently unreachable, thus prevents VLC systems from achieving highspeed transmission [Kar 2015]. In terms of SNR (Signal to Noise Ratio), visible light 
communication systems have a high SNR [Mat 2019]. A common method to increase the modulation bandwidth of LED luminaires is to add an optical filter in the receiver. In summary, VLC systems are characterized as having a low bandwidth with a very high SNR. Therefore, there are several techniques that can be used to obtain a high data rate such as, in 2015, using a cascaded bridged-T equalizer circuit and a blue filter, Huang increased the $3 d B$ bandwidth to $366 \mathrm{MHz}$ and realized a $1.6 \mathrm{Gbit} / \mathrm{s}$ offline VLC transmission using the quadrature amplitude modulation with orthogonal frequency division multiplexing (16QAMOFDM) [Hua 2015]. In 2016, feed-forward pre-equalization is proposed to be used in wireless VLC systems in conjunction with PAM modulation schemes in order to overcome the limited bandwidth of LEDs and achieve an error-free $\left(B E R<10^{-12}\right) 1.6 \mathrm{~Gb} / \mathrm{s}$ and $2 \mathrm{~Gb} / \mathrm{s}$ PAM-4 data transmission are achieved over a free-space VLC link using a 2-tap and3tap feed-forward pre-equalizer respectively [Li 2016], and in 2020, a real-time NRZ-OOK VLC system based on a single commercially available phosphorescent white LED in the VLC systems was realized, the experimental results showed that the $3 d B$ bandwidth of the VLC link with a blue filter was extended from $30 \mathrm{MHz}$ to $600 \mathrm{MHz}$, and the highest real-time data rate of $1.35 \mathrm{~Gb} / \mathrm{s}$ was achieved at the BER of $8.8 \times 10^{-5}$, which was below the forward error correction limit of $3.8 \times 10^{-3}$ [Min 2020].

Another important challenge for VLC systems is to provide a reliable uplink (UL), as their channels are naturally downlink (DL) channels. The use of the same Visible Light (VL) band on both the uplink and the downlink can lead to considerable interference. Therefore, a mechanism must be used to separate the two beams while being able to communicate without interference. This can be achieved by using several techniques [Kli 2017] either Time Division Duplexing (TDD) [Tiw 2016] or Frequency Division Duplexing (FDD) [Zha 2020].

One of the fundamental requirements for VLC systems to complement RF networks in the indoor environment is the mobility. As users of mobile wireless devices move around within the coverage area, VLC systems must provide an uninterrupted link for these users. Since the light beam follows a straight path, mobile users lose their connections. For this, several solutions have been proposed. In [Mmb 2016], a Diffuse Optical Wireless (DOW) configuration has been proposed to solve this problem. On the other hand, VLC systems are built upon the illuminance infrastructure, and their performance is coupled with illumination function. The most common link configurations for indoor OWC or VLC systems in a typical room are the line-of-sight (LOS) and the diffuse or a hybrid LOS-diffuse. Normally, the diffuse system provides a lager coverage area and an excellent mobility [Wu 2011], but at the cost of lower data rates, higher path losses and multipath induced intersymbol interference (ISI) caused by the signal reflections from walls and other objects within the room. On the other hand, LOS links, where the beam is confined within a narrow field of view (FOV), offer a much higher channel capacity and a longer range [Kum 2019]. However, LOS links offer a limited coverage area as well as requiring alignment and/or tracking to maintain link availability. Hence, the transmitter must be designed to have a wide FOV since multiple reflections are allowed from walls and other materials, as shown in the following figure. 


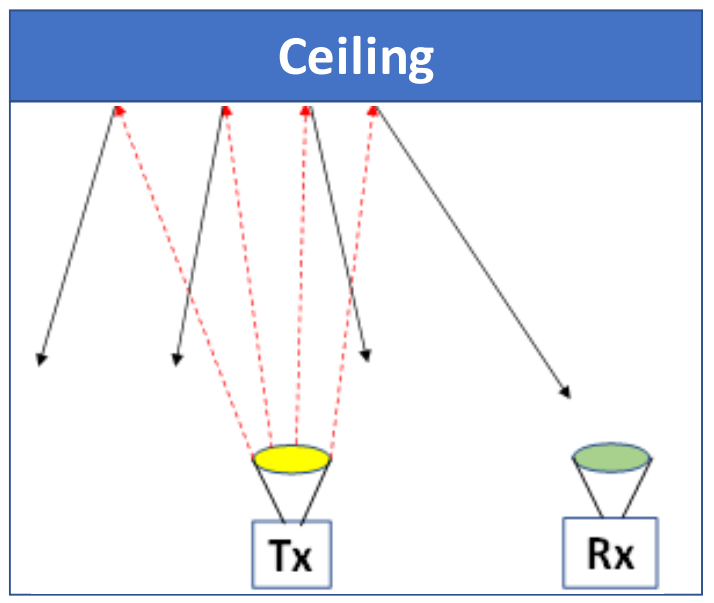

Fig. 1.4: DOW configuration.

Therefore, if a user occupies a transmission channel of the transmitter, reflections from other channels will still be able to reach the receiver and thus enable uninterrupted communication. However, one of the main disadvantages of the DOW configuration is a multipath dispersion. Another challenge for VLC systems is to cooperate with another wireless standard such as RF. The work in [Hou 2006], studied the combination of a high-speed downstream link of a low-speed RF wireless LAN, and showed that the combined system has some advantages in terms of latency and data rate. In [Kas 2018], a parallel communication system of RF and PLC/VLC is proposed showing that the system can be considered as a promising architecture to deploy an indoor wireless network which combines multiple VLC and RF transmitters to enhance rate and reliability of indoor wireless networks.

Both VLC and RF communication technologies are used for wide variety of applications for wireless communication. VLC uses light as medium for communication whereas RF uses electromagnetic (EM) waves. Different from traditional RF systems where phase or frequency modulation with coherent detection is adopted widely, the short range optical wireless communication systems we focus on usually utilize intensity modulation / direct detection (IM/DD). Table 1.2 show a comparison between VLC and RF with regards to various features. 
Table 1.2: Comparison between VLC and RF.

\begin{tabular}{|l|l|l|}
\hline \multicolumn{1}{|c|}{ Feature } & \multicolumn{1}{|c|}{ VLC } & \multicolumn{1}{c|}{ RF } \\
\hline Power Consumption & Less, it is power efficient system. & $\begin{array}{l}\text { High, it is power inefficient } \\
\text { system. }\end{array}$ \\
\hline BW regulated & Yes & No \\
\hline EM interference & $\begin{array}{l}\text { VLC communication is not } \\
\text { affected due to EM sources. }\end{array}$ & $\begin{array}{l}\text { RF communication is affected } \\
\text { due to EM sources. }\end{array}$ \\
\hline Infrastructure & LED illumination & Access point \\
\hline Line-of-sight (LoS) & Yes & No \\
\hline Visibility security & Yes & No \\
\hline Distance & Narrow, short & Wide, medium \\
\hline
\end{tabular}

Radio and optical wireless are complementary transmission media, with different applications favoring the use of one medium over the other. Radio is preferred in applications where user mobility must be maximized or transmission through walls or over a long range is desired. Optical wireless is advantageous for short to medium-range applications in which bit rate perlink and aggregate system capacity must be maximized at minimal cost or receiver signalprocessing complexity. The applications, advantages and limitations of OWC are described in Chapter 3.

\subsubsection{Broadband sources in fiber communications}

Since early 90's, the employment of Broadband sources (BBS) have been of huge technical interest and has been largely suggested as a cost-effective alternative to the expensive use of multiple lasers as WDM light sources [Kim 2007, Gra 2009, Gra 2013]. The fundamental motivation for using incoherent sources is their essentially character and their costeffectiveness for the construction of several narrower WDM channels where the generation of optical channels is done by means of the spectral slicing technique. In this way, implementation and maintenance costs are reduced and the control of temperature stabilization is relaxed. The following figure illustrates the schematic diagram of the operating principle of the spectral slicing technique [Gra 2013].

\section{Transmitter}

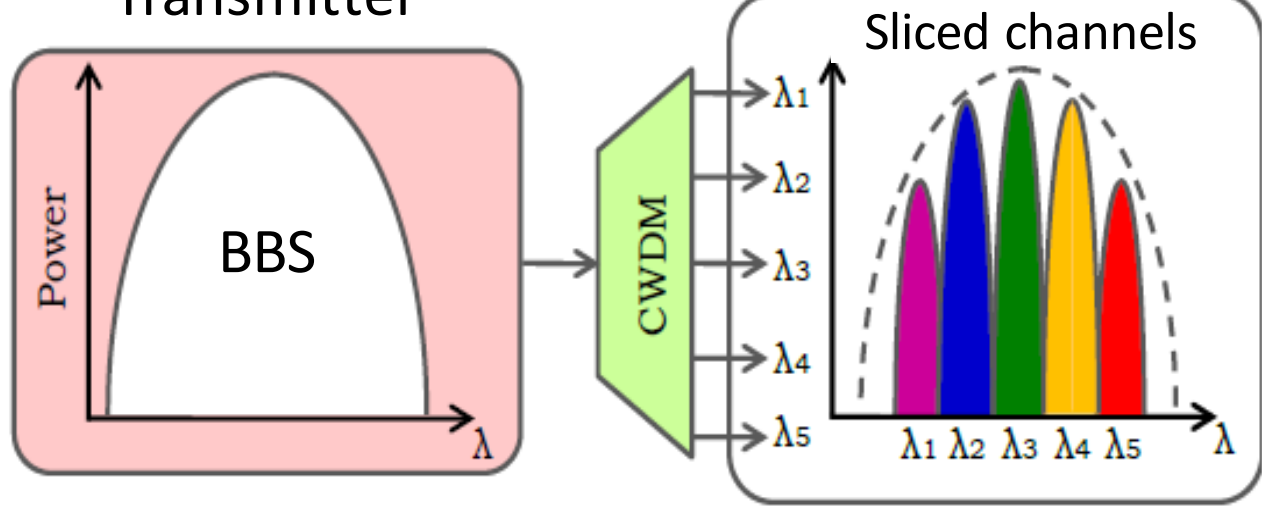

Fig. 1.5: Principle of spectral slicing technique [Gra 2013]. 
As shown in Fig. 1.5, Spectrum slicing technique is one of basic techniques available in WDM-PON (Wavelength Division Multiplexing -Passive Optical Network) systems in order to reduce the cost of components and simplify the passive network architecture. Then, the incoherent BBS is sliced, and equally spaced multi-wavelength channels is generated [Sun 2008]. All cut slices match the wavelength grid defined by the WDM filter and can be used as optical carriers. For this purpose, the Full Width at Half Maximum (FWHM) spectral width of the BBS must be at least equal to the wavelength band of the band-pass filter. There are several types of broadband optical sources and the most typical are white sources such as (super-luminescent) light-emitting diodes (LEDs) and amplified spontaneous emission (ASE) noise spectrum emitted either by an Erbium Doped Fiber Amplifier (EDFA) or a Semiconductor Optical Amplifier (SOA). The spectral slicing technique can be employed to perform both the generation of downlink and uplink carriers in fiber communication [Sek 2018].

Broadband sources with high output power are useful for many applications, such as optical communications, especially fiber communications. Several works on optical transmission using a broadband source, for example, in 2010, [Gra 2010] has demonstrated experimentally a novel photonic transceiver based on the phase modulation of an BBS suitable for RoF (Radio over Fiber) signal transport over $20 \mathrm{Km}$ of SMF (Single Mode Fiber), and the authors deduce that the proposed approach shows a great suitability for WDM networks based on RoF signal transport and also represents a cost-effective solution for passive optical networks. In 2016, [Fal 2016] proposes an integrated single-mode fiber and visible light communication system based on a hybrid Hadamard/OFDM using a LED as the emission source, and the authors deduce that the proposed system is effective in improving the performance of the single-mode fiber and visible light communication system and its low cost due to its simple design in physical layer and would be a feasible solution for indoor internet, Fiber-tothe-Home, smart home based on VLC. In 2017, [Chi 2017b] has demonstrated optical OFDM transmission using an optical broadband source over a $10 \mathrm{~km}$ fiber link, and a transmission of $2 \mathrm{Gbit} / \mathrm{s}$ is obtained using a 3 to $7 \mathrm{~nm}$ broadband ASE optical source without power fading, he concludes that. The proposal is easily adaptable to WDM access networks and compatible with multi-band next generation OFDM-PONs. In 2019, [Sen 2019] has combined a 32-channel spectrum sliced wavelength-division-multiplexed passive optical network (SS-WDM PON) transmission system with a 7-Fiber Bragg gratings (FBG) optical sensor system with one shared broadband light source (BLS) over one optical fiber, acceptable results were achieved and conclusion led to assess that FBG optical sensors network do not negatively affect the SS-WDM PON data transmission system nor such collaboration between both technologies.

\subsection{Objectives}

According to previous section, data communication via LEDs has become an attractive topic and several researchers are continuously researching and proposing techniques to improve the transmission rate and distance, using a broadband source such as LED as an optical transmitter source and different receivers such as photodiodes, cameras or solar cells. 
The general objective of this thesis is the study and practical design of communication systems based on broadband sources for fiber and free space optical links with optimizing the maximum transmission capacity and provided services. Moreover, this objective can be broken down in the following specific tasks (i.e. subobjectives):

Firstly, a theoretical study based on both the bibliography and the most recent publications has been carried out throughout the process of doctoral studies, and it has been continuously updated due to the frequent novelties introduced by the leading research groups over the years.

Since this is a Doctoral Thesis focused on the experimental part, it has been necessary to know the equipment used for the design of the transmission system in visible light as well as the equipment in the Photonics Laboratory to design the transmission system in fiber at $1550 \mathrm{~nm}$ and to acquire the skills derived from its use as well as from the optical components that are referenced throughout this report.

To carry out a simulation study to know the different electronic and optical properties of the semiconductors. The choice of the component used to manufacture LEDs in order to use them as broadband optical sources emitting visible light. In fact, this choice depends on the wavelength of the desired emission which is related to the deviation value of the material and its nature. The generation and reception of digital data employing LEDS as optical broadband sources for emission, and the evaluation of its performance according to several parameters such as LED characteristics, amongst others. The design of a VLC system using a broadband optical source which is a LED, and the evaluation of the performance. This thesis will demonstrate two prototypes of VLC transmission in analog and digital transmission for the transmission of sound, text and image respectively.

The experimental generation and reception of the OFDM optical signal employing an optical broadband source in the third optical communications window for optical fiber transmission and evaluation of performance. More concretely, a low-cost WDM-OOFDM network based on a centralized source by employing double sideband modulation and optical carrier re-use for downlink and uplink will be presented and implemented experimentally.

\subsection{Organization of the thesis}

This doctoral thesis is organized into five chapters where the first chapter provides a general introduction to communication systems based on broadband light sources both for free space and fiber optic links. It summarizes the main information related to the different axes and issues that will be dealt with in the context of the research works included in this thesis.

The chapter two is oriented towards a detailed study of the optoelectronic properties of semiconductors, followed by an explanation of how to design optoelectronic materials using the WIEN2K software. Then, the electrical and optical proprieties and analysis of the $\mathrm{ZnSeO}$ material will be presented. In this research work, we propose the $\mathrm{ZnSeO}$ as an optoelectronic device for optical wireless communication systems. This choice will be corroborated by the simulation results. 
In the third chapter, we will focus on the study of wireless optical communication systems especially the types, configurations and modulations. Two simple experimental prototypes will be designed and presented. The first one is designed for analog data transmission using visible light as well as the wireless power transmission. The second experimental prototype deals with numerical data transmission in simplex mode and half duplex. The main results and challenges will be discussed.

In chapter four, we will focus on transmission via SSMF using OFDM optical and a broadband source. First, we will start by describing the main generalities of broadband sources. The Mach-Zehnder Interferometer (MZI) with Variable Delay Line (VDL) transmission structure is proposed to overcome the limitations of broadband sources in the transmission of optical signals. Next, the generation and reception of OFDM is described sequentially and exhaustively, from the origin of the bits to their reception. Finally, we will present a WDM-OOFDM access network using a centralized optical transceiver based on a single optical broadband sliced source at the Central Office as a low-cost solution offering downlink and uplink signal transmission by using double sideband modulation and optical carrier reuse, as well as dynamic bandwidth allocation, multiple band selection and tunable OFDM band selection in reconfigurable networks at moderate bitrates.

Finally, Chapter five will include a summary of the main results achieved throughout this thesis and a presentation of the key conclusions drawn from the work carried out. Likewise, future lines of research will be addressed for further work. 
Chapter 2

\section{Optoelectronic for optical wireless communications}




\subsection{Introduction}

Optical wireless communication is an emerging field of telecommunications that uses different optoelectronic devices such as light emitting or light detecting devices. Recently, a large number of researchers as [Vuc 2009, Cho 2013, Hua 2015, Kar 2015, Li 2016, Tia 2017, Li 2017, Gao 2019, Yeh 2019, Zho 2019] are focusing on the development of new devices that will adapt to the next generation of broadband optical wireless communication technologies. This future evolution is based on the maturity of the broadband wireless personnel mobile and the ultra-speed fiber communication technologies. Indeed, optical communications spawned a number of developments in optoelectronics, leading to devices like optical modulators, semiconductor optical amplifiers, and avalanche photodiode and vertical-cavity surface-emitting lasers [Eis 2002, Bour 2012].

However, the great development of optoelectronic devices is a direct result of technological advancement in the field of semiconductors. In fact, semiconductors materials allow handling of current and light and their interaction in the same device. In this chapter, we are interested on semiconductors with the energy of gap between visible and ultraviolet light domains because they are good compounds for different optoelectronics devices especially those applied to optical communications.

The proprieties of semiconductors from the $I I-V I$ family of periodic table of elements (Mendeleev Table) are generally similar to those of group $I I I-V$ and $I V$, where, their energy of gap is between visible and ultraviolet light domains. For example, the following alloys $\operatorname{In}_{1-x} A l_{x} N, \operatorname{GaN}_{1-x} A s_{x}$ and $Z n S e_{1-x} O_{x}$ are high efficiency semiconductors for both lighting and solar cells. Their bandgap can be controlled by the visible electromagnetic energy spectrum by varying the stoichiometric coefficient $x$.

In this part of the thesis, we were focusing on studying the mono-zinc chalcogenide, which is an attractive material for many optoelectronic applications [Boua, 2016], and his variants such as zinc selenide $(Z n S e)$ and zinc oxide $(\mathrm{ZnO})$ [Lin, 2010]. The objective of this study was to analyse the performance of direct gap $I I-V I$ semiconductors with cubic zinc-blende phase. Their electronic and optical properties were discussed in [Sek 2016].

The organization of this chapter is as follows: firstly, the optoelectronic proprieties of semiconductors will be presented followed by an explication of how to design the optoelectronics materials using WIEN2K software. Main steps and interconnection of the subprograms will be specified. Then, the electrical and optical analysis of the proposed $\mathrm{ZnSeO}$ material as optoelectronic devices for wireless communication will be detailed. Finally, main observations will be summarized in the conclusion.

\subsection{Optoelectronic proprieties of semiconductors}

Optoelectronics is considered as sub-domain of photonics. It includes study, conception and manufacture of devices that have the ability to transform the electrical energy to optical energy (light) and the optical energy to electrical energy. Optoelectronic devices can work on infrared, visible or ultraviolet spectral regions [Wong, 2000, Xu, 2008, Komi, 2004]. 
The optoelectronic proprieties are a result of the effects of quantum mechanics of light on materials, especially semiconductors. In this part, a general presentation of semiconductors and theirs optoelectronics properties will be presented.

\subsubsection{Semiconductors}

A semiconductor is a solid substance that has conductivity between that of an insulator and that of most metals, either due to the addition of an impurity or because of temperature effects. The study of semiconductors provides a detailed view of their physical properties, and thus increases their potential for technological applications. There are several families of semiconductors, but in this chapter only $I I-V I$ semiconductors will be presented.

The semiconductors are of great interest because of their absorption and fluorescence properties. They can be used as a source of photons for the production of light-emitting diodes [Bour, 2012], lasers [Eisl, 2002] or solar cells [Patta, 2010]. Compounds $I I-V I$ constitute a particular class of semiconductor materials due to their specific physical properties. Up to now their development has been mainly linked to the need for infrared detection and imaging in atmospheric transmission windows of $8-12 \mu \mathrm{m}$ and $3-5 \mu \mathrm{m}$, and more recently in the wavelength range corresponding to optical fiber transmissions.

Recently, many works like as [Her 2002, Nab 2003, Lin 2010, Che 2013, Bou 2016] have been carried out on the study of $I I-V I$ materials and their ternary and quaternary alloys, with a view to their application in telecommunication field, especially optoelectronic components such as LEDs, detectors and solar cells.

The following table illustrates the different commercial applications for optoelectronic devices.

Table 2.1: Commercial applications for optoelectronic devices [Abde, 2006].

\begin{tabular}{|c|l|l|}
\hline Materials & \multicolumn{1}{|c|}{ Devices } & \multicolumn{1}{|c|}{ Applications } \\
\hline GaAs/AlGaAs & Detectors, LED's, infrared, lasers & $\begin{array}{l}\text { TV remote controls, solar energy conversion, } \\
\text { fiber optic communications, system (local } \\
\text { network). }\end{array}$ \\
\hline $\mathrm{InP}$ & Solar cells & Space solar cell \\
\hline $\mathrm{InP} / \mathrm{InGaP}$ & Infrared LEDs, lasers $(1-1.6 \mu \mathrm{m})$ & Fiber optic communications \\
\hline $\mathrm{InP} / \mathrm{InGaAs}$ & $1-1.67 \mu \mathrm{m}$ Detectors & Fiber optic communications \\
\hline $\mathrm{Si}$ & Detectors and solar cells & Solar energy conversion, calculators, detectors \\
\hline $\mathrm{Ge}$ & Detectors & Detectors light \\
\hline $\mathrm{ZnSe} / \mathrm{ZnS}$ & Visible LEDs & $\begin{array}{l}\text { Commercial applications, Visible } \\
\text { communication }\end{array}$ \\
\hline
\end{tabular}




\subsubsection{Semiconductor alloys}

Wide gap $I I-V I$ semiconductors such as $Z n S e$ and $Z n O$ have been studied for several years because they have promising optoelectronic features. Their alloys are classified into several groups according to the number of constituents, so their classification is as follows:

- Binary alloy of the form $\mathrm{AB}(\mathrm{ZnO}$ and $\mathrm{ZnSe})$;

- Ternary alloy: when the binary elements $\mathrm{AB}$ and $\mathrm{AC}$ are combined, the alloy formed can be either:

$>$ Anionic ternary alloy $A B_{x} C_{1-x}$;

$>$ Cationic ternary alloy $A_{x} B_{1-x}$.

These alloys are characterized by the presence of the stoichiometric coefficient $x$.

However, there is also another type of semiconductor alloy called quaternary. This type of alloy involves four binary compounds and two stoichiometric coefficients $x$ and $y$, either of the form $A_{1-x} B_{x} C_{y} D_{1-y}, A B_{x} C_{y} D_{1-x-y}$ (anionic) or $A_{x} B_{y} C_{1-x-y} D$ (cationic).

\subsubsection{Properties of II-VI semiconductors}

In this part of work, only the electronic and the optical properties of II-VI semiconductors will be discussed.

\subsubsection{Electronic properties}

The $I I-V I$ semiconductors are characterized by a large band gap, i. e. a direct forbidden band, corresponding to wavelengths located in the visible up to ultraviolet range. These semiconductor materials have a direct gap ranging from 3.741eV ( $\mathrm{nnS}$ [Bara, 1978]) to $1.525 \mathrm{eV}$ (CdTe [Hern, 2002]). Fig. 2.1 represents the interband transition for a semiconductor.

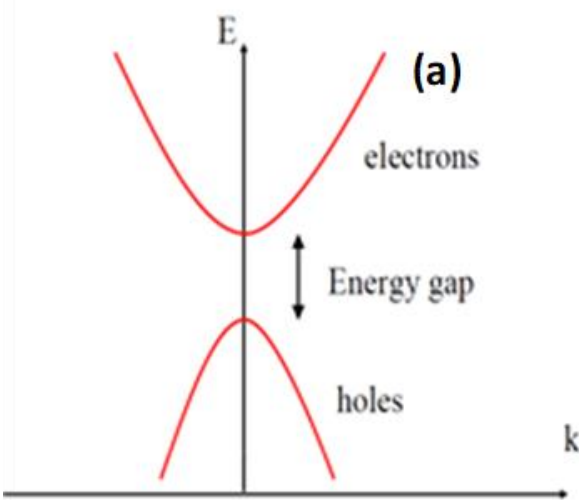

Direct bandgap semiconductors

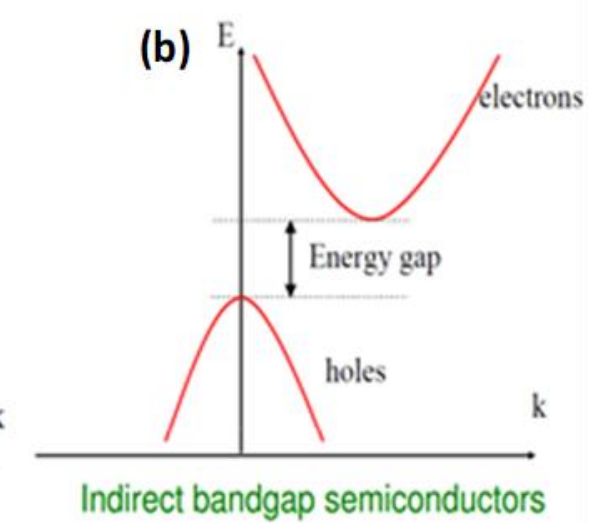

Indirect bandgap semiconductors

Fig. 2.1: Interband transitions for semiconductors. (a) Direct bandgap, (b) Indirect bandgap. 
The band gap (Eg) is important for optoelectronic applications, as it defines the energy of the transition involved in the absorption and emission of light, the forbidden band defines the emission color of light in a LED or solid-state laser and the lowest absorption energy for a photovoltaic device.

\subsubsection{Optical properties}

The optical properties of semiconductors depend on their electronic structure because they involve transitions between different electronic states. This transition can be made without changing the wave vector in direct gap semiconductors, which allows the absorption and emission of light much more efficiently than in materials with indirect gap. Hence, for all industrial applications, the choice of materials depends on the band gap and also the lattice constant to provide uniform heterostructures will reduced defects.

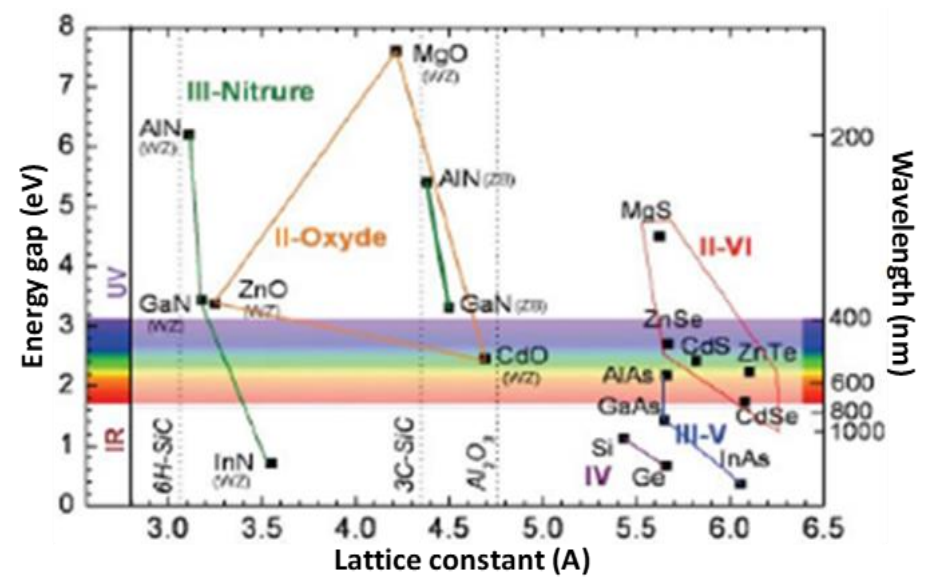

Fig. 2.2: Band gap energy according to the mesh parameter for some semiconductors.

From Fig. 2.2, we can observe that the Zinc-based $I I-V I$ materials can be used for devices operating in the visible to ultraviolet range, such as LEDs and photovoltaic cells.

The optical properties of semiconductors are revealed in the form of interaction of radiation with electrons, which are interpreted according to three processes: absorption, spontaneous emission and stimulated emission. They are described by the real and imaginary part of the dielectric function, the refractive index, the extinction coefficient and the reflectivity.

- Absorption: when the absorbed photon causes an electron jump from an occupied state of the valence band to an empty state of the conduction band, there would be an absorption;

- Spontaneous emission: it occurred when an electron from the conduction band spontaneously falls back on an empty state of the valence band;

- Stimulated emission: if the absorbed photon induces the transition of an electron from the conduction band to an empty state of valence band with emission of a photon, the emission would be stimulated. 


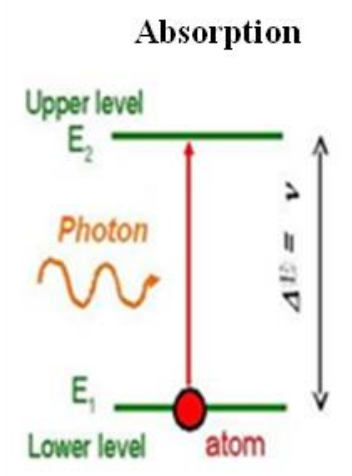

Spontaneous emission

Stimulated emission
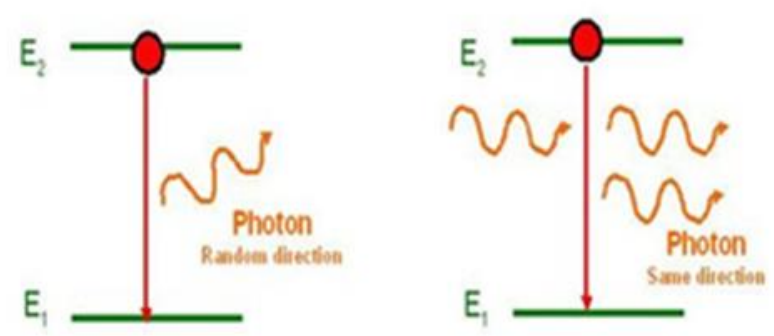

Fig. 2.3: Interaction of radiation and electrons in semiconductors.

\subsection{Design of Optoelectronics Materials}

The design of optoelectronic devices requires a depth study of the materials to be chosen and an appropriate choice of the material simulation software. Hence, several software programs have been created for simulating the optoelectronic materials and devices. WIEN2k software is widely used; we will exploit it in this chapter to study a new material that we have proposed for use in optoelectronic devices such photovoltaic cells and LEDs. A succinct explanation of WIEN2K and its operating principle is also provided.

\subsubsection{WIEN2K software description}

WIEN2k is a software tool programmed in Fortran language that allows to perform quantum calculations on periodic solids. It performs electronic structure calculations of solids using density functional theory (DFT) [Blah 2008]. It is based on the full-potential (linearized) augmented plane-wave (FP-LAPW) plus local orbitals (lo) method, one of the most accurate schemes for band structure calculations. This software was designed by Blaha and his collaborators [Bla 2008].

The Wien $2 \mathrm{~K}$ code is an implementation of the FP-LAPW method. Before describing the WIEN2K software operation, we will give a brief overview of the DFT and FP-LAPW methods.

\subsubsection{The Theory of the Functional Density}

The numerical simulations have acquired a prominent place in physics and particularly in materials science. The density functional theory (DFT) and its resulting improved methods become now important for the study of material properties [Hoh 1964, Koh 1965, Koc 2000]. In fact, all the information related to a system composed of a set of particles, in which there are interactions between positive and negative charges, is contained in its wave function $\psi$ [Har 1928]. This function allows determining a fundamental parameter of the system which is the probability density and $\psi$ is obtained by solving the Schrödinger equation:

$$
\hat{\mathrm{H}} \boldsymbol{\psi}=\boldsymbol{E} \boldsymbol{\psi}
$$


where $E$ is the total energy, $\psi$ is the wave function and $\hat{\mathrm{H}}$ is the Hamiltonian of the system given by the following equation:

$$
\hat{\mathrm{H}}=\boldsymbol{T}_{e}+\boldsymbol{V}_{e-e}+V_{N-e}+T_{N}+V_{N-N}
$$

Where $\boldsymbol{T}_{\boldsymbol{e}}$ is the kinetic energy of electrons, $\boldsymbol{T}_{\boldsymbol{N}}$ is the kinetic energy of the nuclei, $\boldsymbol{V}_{\boldsymbol{N}-\boldsymbol{e}}$ is the potential energy of nucleus-electron attraction, $\boldsymbol{V}_{\boldsymbol{e}-\boldsymbol{e}}$ is the potential energy of repulsion between electrons and $\boldsymbol{V}_{\boldsymbol{N}-\boldsymbol{N}}$ is the potential energy of repulsion between the nuclei.

Different approximations were developed [Bor 1927, Har 1928, Sla 1951] to solve the eq. (2.1). However, it has been proven that the solution is extremely difficult to obtain, even in the simplest cases. Indeed, when the number of atoms increases, the difficulties of calculation increase exponentially [Hoh 1964, Koh 1965].

The functional density theory (DFT) is a good tradeoff and numerically less expensive. It started with the fundamental theorems of Hohenberg and Kohn in 1964 [Hoh 1964] which established a functional relationship between the energy of the ground state and its density. The purpose of the DFT is to describe a system by considering the density as a basic variable [Tho 1927, Fer 1927, Dir 1930].

Generally, the electron density $\rho(r)$ is a positive function depending of only three space variables that tends towards zero when $r$ tends towards infinity and whose integral over the whole space gives the number $N$ of electrons, so that:

$$
\left\{\begin{array}{l}
\lim _{r \rightarrow \infty} \rho(r)=0 \\
\int \rho(r) d r=N
\end{array}\right.
$$

where $\boldsymbol{\rho}(\boldsymbol{r})$ represents the probability to finding one of the $N$ electrons in the volume element $d r$. Hence, electron density, unlike wave function, is an observable that can be measured experimentally (by X-ray diffraction).

However, it is possible to reformulate the problem by using appropriate theorems and approximations [Koh 1965, Perd 1992]. In the following, we will present two standard methods which are mainly used to study the properties of material namely the Local Density Approximation (LDA) and the Generalized Gradient Approximation (GGA) [Cep 1980, Per 1992].

\subsubsection{The Local Density Approximation (LDA)}

The local density approximation (LDA) is a simplest and most common way to calculate the exchange and correlation term. It considers the exchange and correlation potential as a local quantity, defined at a point $r$ which is weakly dependent on density variations around this point [Beck 1988, Perd 1992, Aou 2009, Gér 2013, Ben 2014]. Its corresponding density is $\rho(r)$. This density will be replaced by that of a homogeneous electron gas of the same density $\rho(r)$. The functional of exchange and correlation is expressed as follows:

$$
E_{x c}^{L D A}[\rho(r)]=\int \rho(r) \varepsilon_{x c}^{L D A}[\rho(r)] d^{3} r
$$


where $\boldsymbol{\rho}$ is the electronic density, $\varepsilon_{x c}$ is the exchange-correlation energy per particle of a homogeneous electron gas of charge density $\boldsymbol{\rho}$.

The LDA approximation ignores the exchange-correlation corrections to be made when the electron density is no longer locally homogeneous. However, LDA is particularly effective and its application to atoms and molecules is justified by the success of these numerical applications.

\subsubsection{The Generalized Gradient Approximation (GGA)}

The generalized gradient approximation (GGA) [Per 1992] is another method that overcomes the limitations encountered with LDA. The GGA adds a new part which depends on the density gradient and the correlation exchange term is then formulated as follows:

$$
E_{x c}^{G G A}[\rho(r)]=\int \rho(r) \varepsilon_{x c}^{\text {hom }}[\rho(r), \nabla \rho(r)] d^{3} r
$$

Where, $\varepsilon_{x \boldsymbol{c}}^{G G A}[\boldsymbol{\rho}(\mathbf{r}), \boldsymbol{\nabla} \boldsymbol{\rho}(\mathbf{r})]$ represents the correlation exchange energy per electron in a mutually interacting electron system of non-uniform density. $\boldsymbol{E}_{\boldsymbol{x} \boldsymbol{C}}^{\boldsymbol{G} \boldsymbol{G A}}$ is divided into two contributions which are exchange and correlation:

$$
E_{x c}^{G G A}[\rho, \nabla \rho]=E_{x}^{G G A}[\rho, \nabla \rho]+E_{c}^{h o m o}[\rho, \nabla \rho]
$$

The GGA is given by different parameterizations among them, those of Perdew and these collaborators [Per 1992, Per 1996]. The GGA compared to LDA improves the description of chemical bonds (lengths, binding energies, etc). However, the quality of the results obtained by the LDA and GGA approaches is similar in semiconductors.

\subsubsection{The FP-LAPW method}

Different calculation methods have been developed based on the formalism of the DFT. The LAPW method [Bla 1990, Sin 1994], enables accurate calculations of electronic and magnetic properties of poly-atomic systems using density-functional theory (DFT) [Hoh 1964, Koh 1965]. One successful implementation of the full-potential LAPW (FP-LAPW) method is the program package WIEN, a code developed by Blaha, Schwarz and co-workers [Bla 1990]. In the FP-LAPW method [Sla 1937, And 1975, Sin 1991, Ham 1979, Sjö 2000], no approximation is made for the shape of the potential or the charge density. They are developed into harmonics network within each atomic sphere, and into Fourier series in interstitial regions, which is the origin of the name "Full-Potential".

According to this approximation the system is divided into two regions: The first region describes the spheres centered on the atomic sites in which the radial solutions of the Schrödinger equation are used, the potential and the wave functions are of the Muffin-tin (MT) form with spherical symmetry inside the MT sphere of radius $R_{\alpha}$. The second describes the interstitial region with the basic expansion of plane waves and the potential can be assumed to be constant, so the wave function is written as: 


$$
\Psi(r)=\left\{\begin{array}{lr}
\frac{1}{\sqrt{\Omega}} \sum_{G} C_{G} \mathrm{e}^{i(G+K) r} & r>R_{\propto} \\
\sum_{l m} A_{l m} U_{l}(r) Y_{l m}(r) & r<R_{\propto}
\end{array}\right.
$$

Where $\boldsymbol{R}_{\propto}$ represents the radius of the MT sphere, $\Omega$ is the Volume of the elementary mesh. $\boldsymbol{C}_{\boldsymbol{G}}$ et $\boldsymbol{A}_{\boldsymbol{l m}}$ : are the coefficients of the development in spherical harmonics $\boldsymbol{Y}_{\boldsymbol{l m}}$.

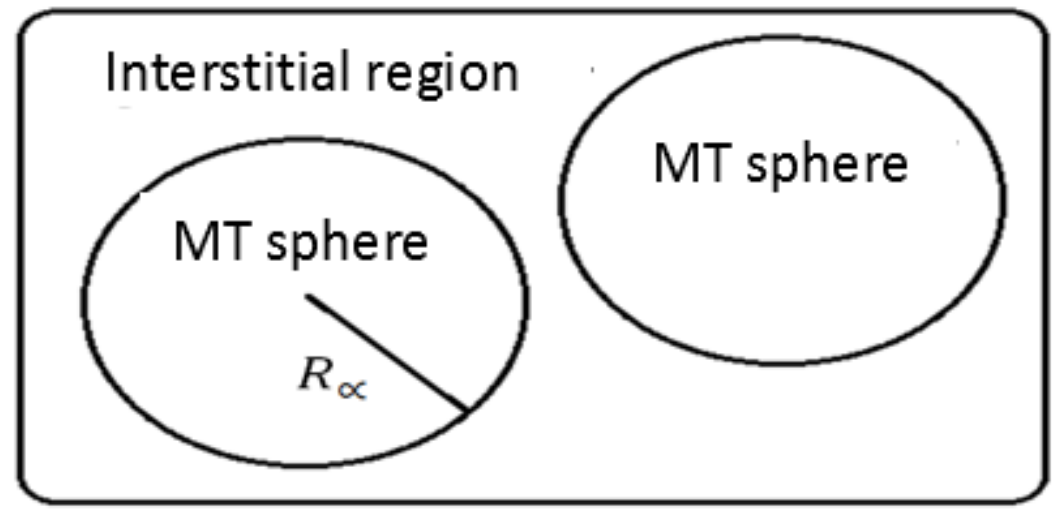

Fig. 2.4: Muffin-Tin potential.

According to the literature [Pet 1999, Baa 2006, Bla 2020], the FP-LAPW method is the most accurate method, but it is time-consuming, mainly because plane waves are not well adapted to describe a wave function. It is therefore necessary to use a relatively large base.

\subsubsection{The flowchart of WIEN2K}

Wien2K is a well-known software used for computation of properties of materials. It is based on the implementation of the Full Potential Linearized Augmented Plane Waves (FP-LAPW) method [Bla 2008]. Wien2K is widely used to compute the electric field gradient [Har 1974, Har 1974b], the high temperature superconducting systems [Know 1974], the minerals [Pen 1962], the surfaces of the transition metal [Sti 1972], the non-ferromagnetic oxides [ Shi 1974], and even molecules [Koh 1995].

Wien2K consists of various independent programs that are linked by C-SHEL SCRIPT. Before the presentation of main steps of computation of different properties of materials, we will start by the calculation of fundamental quantities such as electron density, potential, proper functions and clean energies. The process of calculation in the WIEN2k code takes place in three steps:

\section{Initialization:}

This is the start of the operations carried out by a series of auxiliary programs that generate the symmetry of the geometry of the studied material, the starting densities, the number of special points necessary for integration in the Brillouin irreducible zone...etc. 
$>$ NN: is a subprogram which computes the distances between the nearest neighbors up to a specified limit (defined by a distance factor $F$, usually taken as 2 ) and therefore helps to determine the value of the radius of the atomic sphere. In addition, it controls the overlap of the atomic spheres. The output file of this subprogram is called: case.outputnn;

$>$ SGROUP: determines the space group, as well as the point group of a given structure. The output file is called: case.struct.group;

> SYMMETRY: generates the symmetry operations of the spatial group from the case.struct file, as well as the point group of individual atomic sites;

LSTART: generates the electron densities of free atoms and determines how the different orbitals are treated in band structure calculations (such as core states with or without local orbitals). In addition, this subprogram requires specifying the correlation exchange potential (GGA) and the cut-off energy that separates the core states from the valence states (usually taken as $-\mathbf{6} \boldsymbol{R} \boldsymbol{y}$ );

$>$ KGEN: generates a mesh of $k$-points in the Brillouin area. The number of $k-$ points in the Brillouin area must be specified;

DSTART: produces an initial density for the self-coherent SCF (Self Consistent Field) cycle by overlapping the atomic densities generated by the LSTART subprogram;

$>$ Self-coherent calculation (or self-consistent): Calculations of the energy and electronic density of the ground state made in this step according to the convergence criterion (energy, charge density, force);

$>$ LAPW0 (POTENTIEL): potential generation for density calculation;

DAPW1 (BANDES): launch the calculation of valence bands, eigenvalues and eigenvectors;

LAPW2 (RHO): launch the calculation of the valence densities for the eigenvectors;

LCORE: starts the calculation of states and core densities;

$>$ MIXER: mixes the input and output densities (starting, valence and core densities).

\section{Determination of properties:}

Once the convergence criterion is performed and the self-coherent calculation is completed, one can study the properties of the ground state that are determined (i.e. charge density, band structure, optical properties, etc.). The interconnection of these different subprograms is illustrated in Appendix I.

\subsection{Study of the $\mathrm{ZnSeO}$ material for optoelectronic devices in wireless communication}

The work presented in this section demonstrates the performance of the ternary system $Z_{n S e_{1-x}} O_{x}$ [Nab 2003, Che 2013, Bou 2016] and the related binary system ( $\mathrm{nnSe}$ and $\mathrm{ZnO}$ ) as solid-state lighting, in particular the choice of the component for LED manufacturing to be used as a transmitter/receiver in visible light communication (VLC). In fact, this choice 
depends on the wavelength of the desired emission, which is related to the value of the material deviation and its nature in order to obtain a high efficiency.

The objective of this study is to analyze the performance of $I I-V I$ semiconductors direct gap with cubic phase zinc-blend. Their electronic and optical properties are discussed. The main functional parameters as a function of oxygen concentration are given such as: bandgap, refractive index, dielectric fraction, absorption coefficient, lattice parameter and bulk module. Calculations are performed by deploying Ab-initio method approaches based on functional density theory (DFT) within the LDA and GGA approximations using the Wien $2 \mathrm{~K}$ code which is based on the FP-LAPW method.

The results presented in this section were the subject of a paper published in 2016 [Sek 2016] and a book chapter published in 2018 [Sek 2018a].

\subsubsection{Computational details}

The calculation was done using the code WIEN2K. In the LDA approximation, the electronic density varies slowly inside the system, where in the GGA approximation the energy of the exchange- correlation depends on the gradient. The binary compounds $\mathrm{ZnSe}, \mathrm{ZnO}$ and ternary $Z_{n S e_{1-x}} O_{x}$ crystallize in the cubic structure (Zinc Sphalerite), and they belong to the space group of $F 43 m$. In this work, the structure is chosen to be cubic. The atomic positions in this structure are $Z n(0,0,0)$ and $S e / O(1 / 4,1 / 4,1 / 4)$.

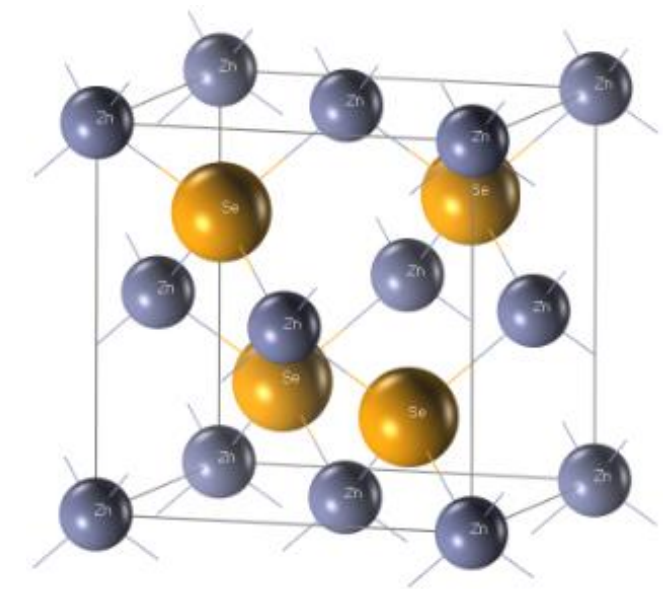

Fig. 2.5: The Zinc Sphalerite structure of a $I I-V I$ binary semiconductor $(Z n S e)$.

The properties of the II-VI compounds will be studied in the first Brillouin zone for a Zinc Sphalerite structure. This first Brillouin zone has the shape of a truncated octahedron. This reduced space of the reciprocal network is characterized by points and lines of particular importance, they are called points and lines of high symmetry and can be described from the group theory. 


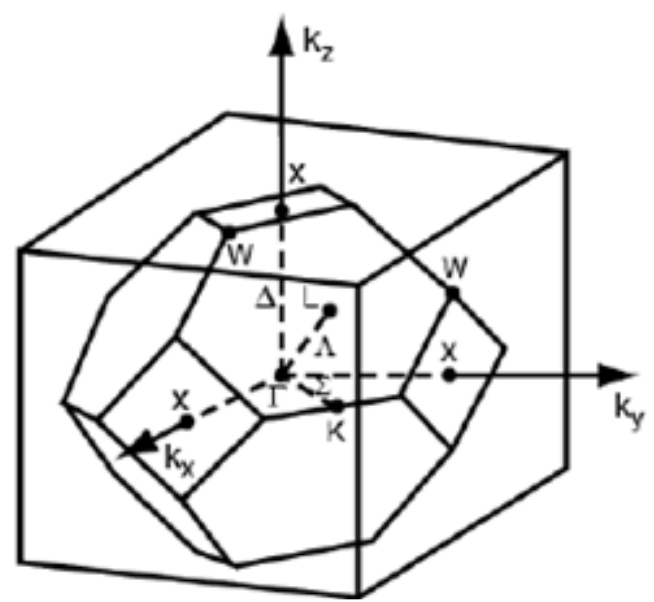

Fig. 2.6: The Brillouin zone for a Zinc Sphalerite structure.

Where $\Gamma, \mathrm{X}, \mathrm{L}, \mathrm{W}, \mathrm{Z}$ are the points of high symmetry and $\Delta, \sum, \Lambda$ are the lines of high symmetry.

$\boldsymbol{\Gamma}$ : This is the center of the first Brillouin zone with the coordinates $\boldsymbol{k}_{\Gamma}=(\mathbf{0}, \mathbf{0}, \mathbf{0})$.

$\mathbf{X}$ : This is the center of a square face of the octahedron that belongs to one of the axes $k_{x}, k_{y}$ or $k_{z}$ with one of the square faces. Hence: $\boldsymbol{k}_{x}=\frac{2 \pi}{a}( \pm \mathbf{1}, \mathbf{0}, \mathbf{0}) ; \boldsymbol{k}_{\boldsymbol{y}}=\frac{2 \pi}{a}(\mathbf{0}, \pm \mathbf{1}, \mathbf{0})$; $\boldsymbol{k}_{\mathrm{z}}=\frac{2 \pi}{a}(0,0, \pm 1)$

$\mathbf{L}$ : This is the center of a hexagonal face of the octahedron whose coordinates are: $k_{l}=\frac{2 \pi}{a}(1,1,1)$

$\mathbf{W}$ : This point is on one of the vertices of the square faces, whose coordinates are: $k_{w}=\frac{2 \pi}{a}\left(0, \frac{1}{2}, 1\right)$

$\mathbf{Z}$ : This point is located on the line that joins the center of a square face to one of the corners of the octahedron with the coordinates $: \boldsymbol{k}_{z}=\frac{2 \pi}{a}\left(\mathbf{1}, \frac{1}{2}, \mathbf{1}\right)$.

$\Delta$ : This line represents the $<\mathbf{1 0 0}>$ direction, and connects the center $\Gamma$ to point $\mathrm{X}$.

$\sum$ : This is a point belonging to the plane of symmetry $k_{x}=k_{y}$ or $k_{y}=k_{z}$ or $k_{x}=k_{z}$.

$\mathbf{\Lambda}$ : This line is the $<100>$ direction, and it connects the center $\Gamma$ to the center of a hexagonal face which is point $L$ of the octahedron.

\subsubsection{The properties of the $\mathrm{ZnSe}_{1-x} \mathrm{O}_{x}$ material}

\subsubsection{Physical properties}

The variation of total energy according to the volume of the component is computed for different values of oxygen concentration $(x)$ ranging from $x=0(\mathrm{ZnSe})$ to $x=1(\mathrm{ZnO})$, using both LDA and GGA approximations. This allows us to determine the lattice constant. The results are shown in Fig. 2.7. 


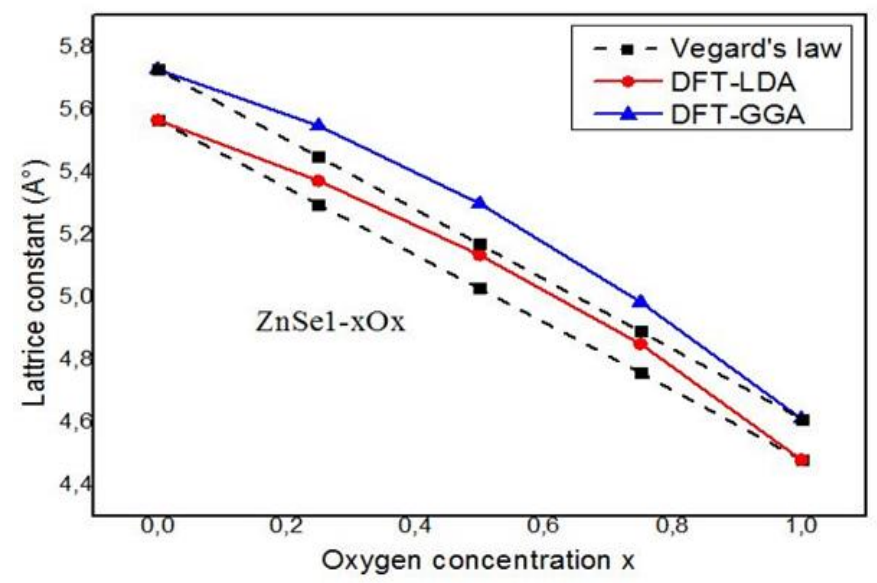

Fig. 2.7: Lattice constant versus oxygen concentration in $Z n S e_{1-x} O_{x}$.According to Fig. 2.7, The lattice constants found using LDA approximation for binary $Z n S e$ and $Z n O$ are $5.5652 \AA$ and $4.48 \AA$, respectively. However, using the GGA approximation, we have found $5.7270 \AA$ and $4.6116 \AA$ for $Z n S e$ and $Z n O$, respectively.

It can be observed that the lattice constant decreases monotonically with the augmentation of the oxygen concentration even if using the DFT-LDA, the DFT-GGA or the law of Vegard.

Another parameter which represents the amount of compressibility of the material named the bulk modulus was calculated for different values of oxygen concentrations from $x=0$ ( $Z n S e)$ to $x=1(\mathrm{ZnO})$ based on the LDA and GGA approximations (Fig. 2.8).

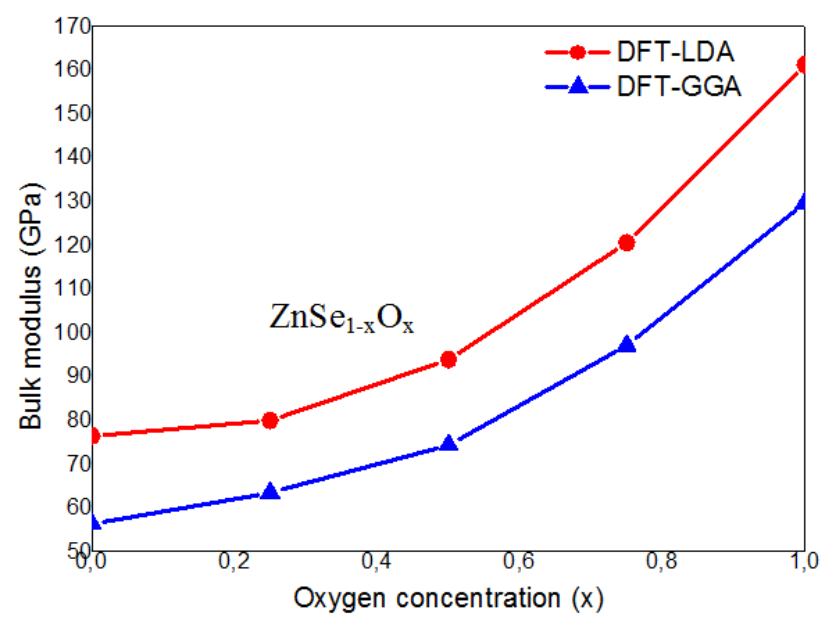

Fig. 2.8: Bulk modulus versus oxygen concentration in $\mathrm{ZnSe}_{1-x} \mathrm{O}_{x}$.

From Fig. 2.8, it can be observed that the amount of compressibility increases with the fraction $x$, therefore one can say that $Z n O$ is more rigid than $Z n S e$, and the ternary compound $Z_{n S e_{1-x}} O_{x}$ becomes less compressible for both LDA and GGA approximations. 


\subsubsection{Electronic properties}

The importance of the electronic properties of a material lies in the fact that they enable us to analyze the nature of the connections which are formed between the various elements of material.

Fig. 2.9 shows the energies of the gap versus oxygen concentrations computed for the $\mathrm{ZnSe}_{1-x} \mathrm{O}_{x}$ compound based on the LDA and GGA approximations.

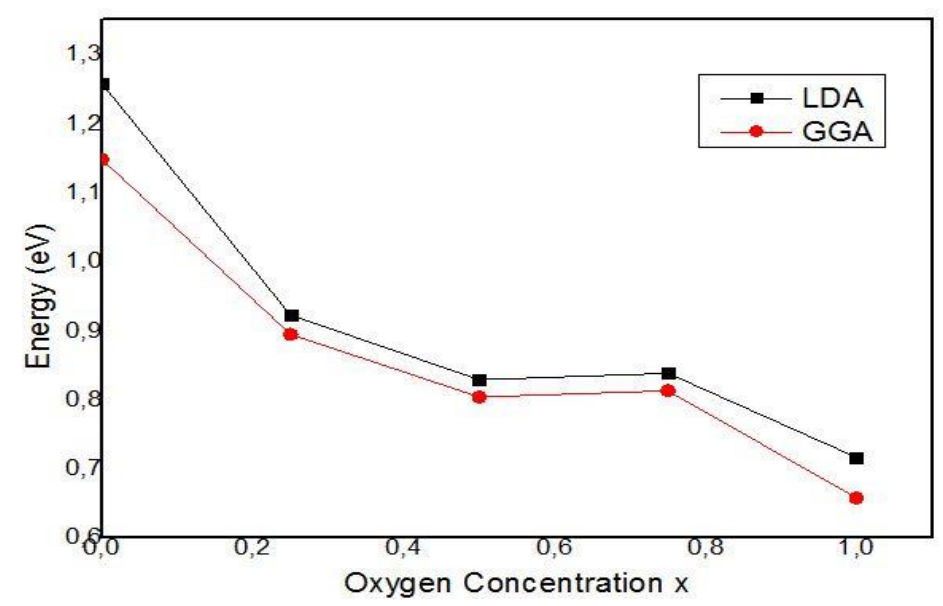

Fig. 2.9: Energy of gap versus oxygen concentration in $\mathrm{ZnSe}_{1-x} \mathrm{O}_{x}$.

As shown in Fig. 2.9, it can be observed that the $\mathrm{ZnSe}_{1-x} \mathrm{O}_{x}$ alloys have a significant bandgap reduction with $x$ increase, the corresponding wavelengths at these energies are in the infrared spectral region, also, the oxygen induced in $\mathrm{ZnSe}$ modifies the conduction band structure.

\subsubsection{Optical properties}

In this section, a review of the main optical properties for the ternary compounds $Z n S e_{1-x} O_{x}$ will be studied. The optical features of the semiconductors are very valuable for the quantitative evaluation of the electronic band structure. Thus, the optical reflectivity, transmission and refraction contribute to determine the dielectric function of the solid.

\section{- The dielectric function}

The dielectric function is related to the optical conductivity which can be represented by the electrical conductivity in the presence of an alternating electric field. Therefore, the dielectric function $\varepsilon(\omega)$ can be formulated as follows:

$$
\varepsilon(\boldsymbol{\omega})=\varepsilon_{1}(\boldsymbol{\omega})+\mathrm{i} \varepsilon_{2}(\boldsymbol{\omega})
$$

where $\varepsilon_{1}(\boldsymbol{\omega})$ and $\varepsilon_{2}(\boldsymbol{\omega})$ are, respectively, the real and imaginary parts of the dielectric function. 
To determine the optical properties of the ternary compounds $Z n S e_{1-x} O_{x}$ and their binary compounds $\mathrm{ZnSe}$ and $\mathrm{ZnO}$, we have used the parameter of the optimized network obtained in the calculations of the structural properties. Also, the number of special points in the first zone of Brillouin is increased in order to detect all possible optical transitions. Then, the LDA and GGA approximations are applied to identify the potential of exchange and correlation.

The results of the real and imaginary parts of the dielectric function under a lower radiation of $30 \mathrm{eV}$ for $\mathrm{ZnSe}_{1-x} \mathrm{O}_{x}$ compounds to the Zinc Sphalerite are shown in Fig. 2.10, based on the LDA and GGA approximations.

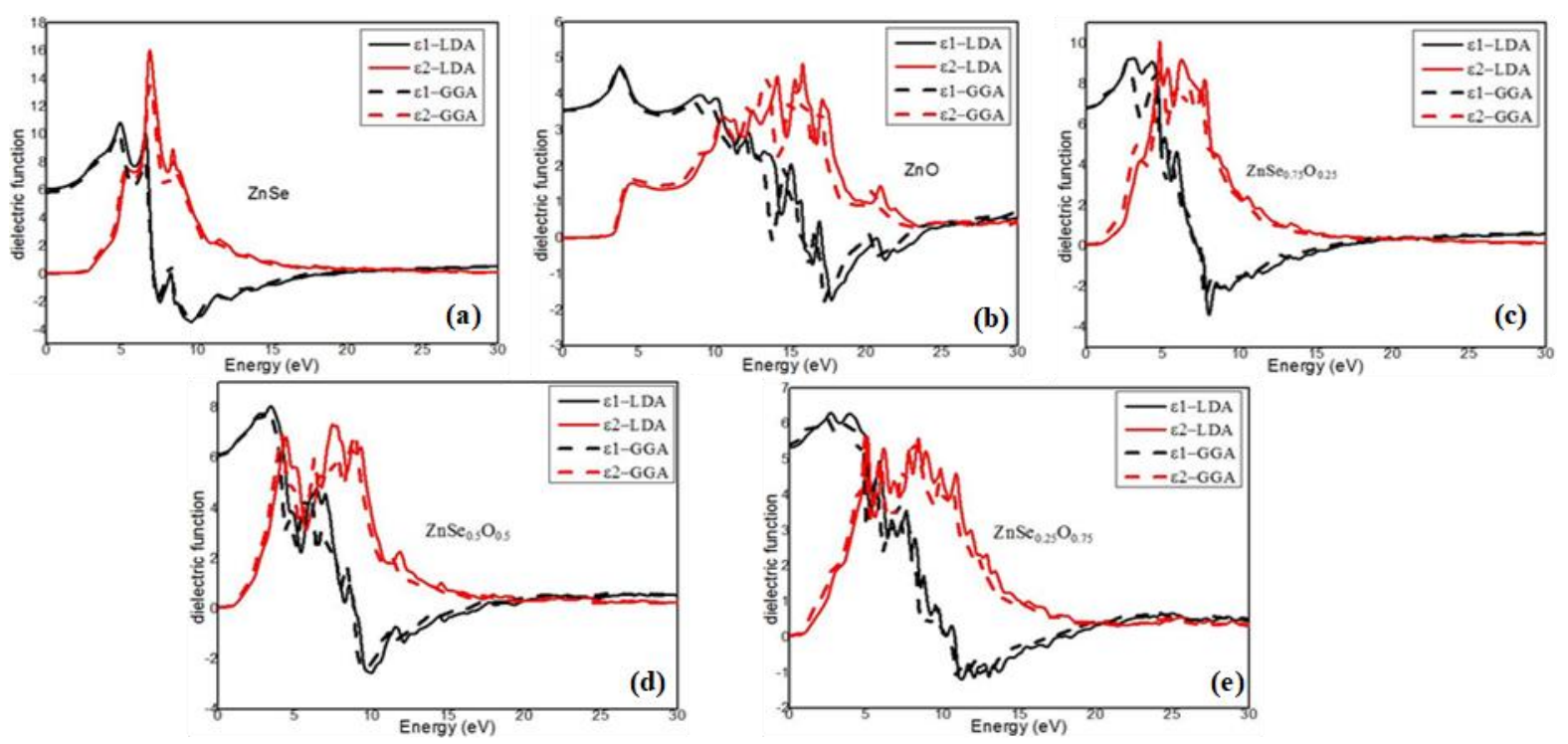

Fig. 2.10: The real and imaginary parts of the dielectric function of $Z n S e_{1-x} O_{x}$ compound to the structure Zinc Sphalerite based on LDA and GGA approximations: (a) $Z n S e$, (b) $Z n O$, (c) $Z n S e_{0.75} O_{0.25}$, (d) $Z n S e_{0.5} O_{0.5}$, (e) $\mathrm{ZnSe}_{0.25} \mathrm{O}_{0.75}$.

According to the results obtained in Fig. 2.10, we can observe that the behavior of the real part of the dielectric function $\varepsilon_{1}(\omega)$ remains the same for all the $Z n S e_{1-x} O_{x}$ compounds. It reaches its maximum peaks of about $4.8 \mathrm{eV}$ for $\mathrm{ZnSe}$ (Fig.2.10a), 3.7eV for $\mathrm{ZnO}$ (Fig.2.10b), $3.07 \mathrm{eV}$ for $\mathrm{ZnSe}_{0.75} \mathrm{O}_{0.25}$ (Fig.2.10c), $3.34 \mathrm{eV}$ for $\mathrm{ZnSe}_{0.5} \mathrm{O}_{0.5}$ (Fig.2.10d) and $2.65 \mathrm{eV}$ for $Z n S e_{0.25} O_{0.75}$ (Fig.2.10e) for both LDA and GGA approximations, then, the curve (a) made a steep decrease between $7 \mathrm{eV}$ and $7.5 \mathrm{eV}$ for $\mathrm{ZnSe}$, when the curve (b) of $\mathrm{ZnO}$ starts decreasing from $4.99 \mathrm{eV}$ till $17.5 \mathrm{eV}$, those of the $\mathrm{ZnSe}_{0.75} \mathrm{O}_{0.25}, \mathrm{ZnSe}_{0.5} \mathrm{O}_{0.5}$ and $\mathrm{ZnSe}_{0.25} \mathrm{O}_{0.75}$ decrease from around $5 \mathrm{eV}$ till $7 \mathrm{eV}, 10 \mathrm{eV}$ and $11 \mathrm{eV}$ for both LDA and GGA approximations, respectively. Here, the dielectric constant reaches its minimums negative values and tends to reach zero level at higher energies.

From the imaginary part of the dielectric function $\varepsilon_{2}(\omega)$, we can easily deduce the fundamental gap at equilibrium which corresponds to threshold energy. As shown in Fig. 2.10 , the first onset of the direct transition is around $0.93 \mathrm{eV}$ for $Z n S e_{0.75} O_{0.25}, 0.88 \mathrm{eV}$ for $\mathrm{ZnSe}_{0.5} \mathrm{O}_{0.5}$ and $0.814 \mathrm{eV}$ for $\mathrm{ZnSe}_{0.25} \mathrm{O}_{0.75}$ using both LDA and GGA approximations. It can be observed from the curves that the number of the sharp peaks on energies depends on the oxygen concentration in the ternary allows. 


\section{- Refractive index}

The dielectric function can be computed using another important optical feature namely the complex index of refraction which is related to the microscopic atomic interactions. Under the same conditions of simulation, the refractive index for $Z n S e_{1-x} O_{x}$ compounds to the zinc sphalerite are presented in Fig. 2.11 based on the LDA and GGA approximations.

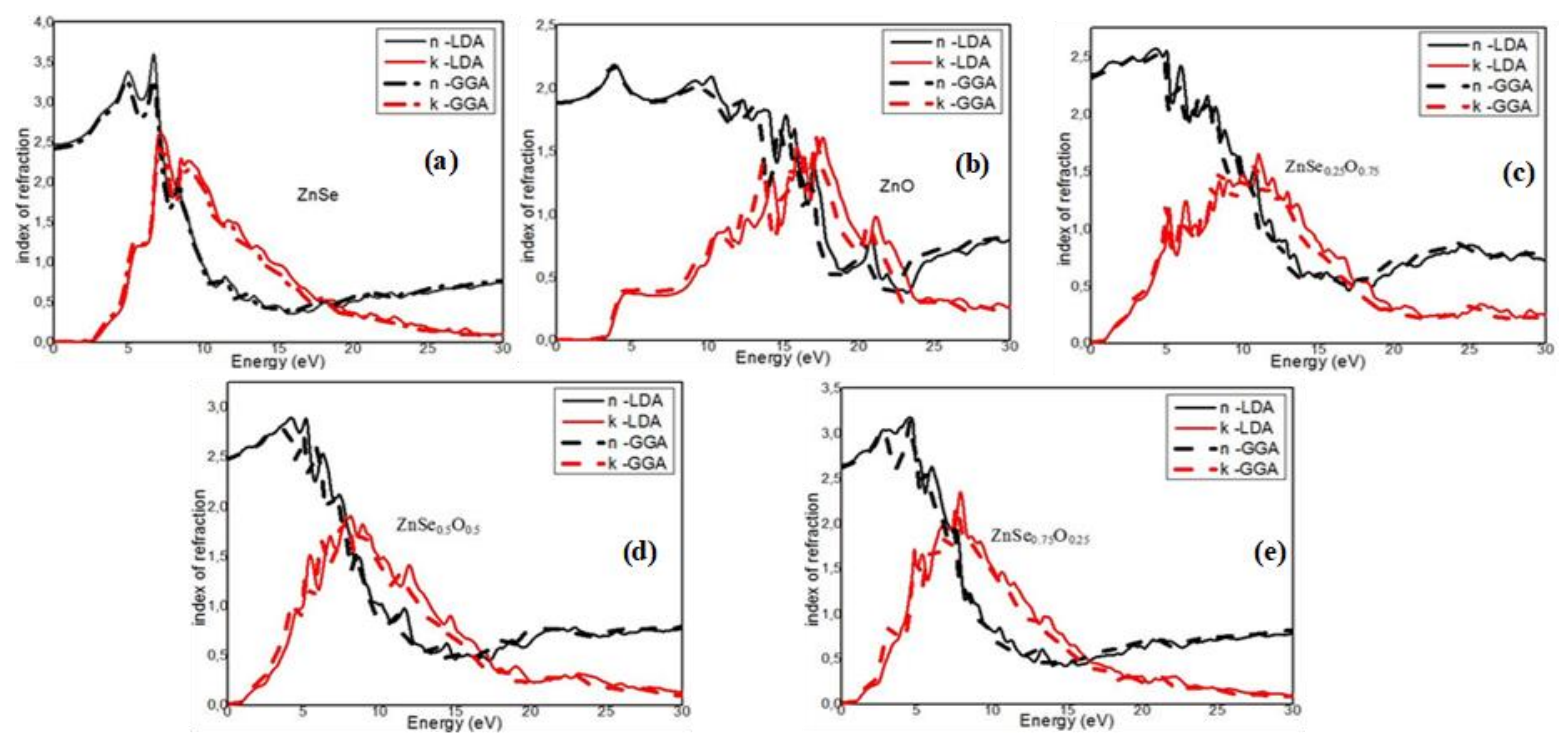

Fig. 2.11: The real $(n)$ and imaginary parts $(k)$ of the index of refraction of the ternary compound $Z n S e_{1-x} O_{x}$ for the structures zinc sphalerite based on LDA and GGA approximation. (a) $Z n S e$, (b) $Z n O$, (c) $Z n S e_{0.75} O_{0.25}$, (d) $\mathrm{ZnSe}_{0.5} \mathrm{O}_{0.5}$, (e) $\mathrm{ZnSe}_{0.25} \mathrm{O}_{0.75}$.

In Fig. 2.11, the index of refraction of the ternary compounds $Z n S e_{1-x} O_{x}$ is presented. For $\mathrm{ZnSe}$, the maximum of energies is reached for around $6 \mathrm{eV}$ and $4 \mathrm{eV}$ using LDA and GGA approximations, respectively, however, for $\mathrm{ZnO}$ and $\mathrm{ZnSe}_{1-x} \mathrm{O}_{x}$, the values are around $3.7 \mathrm{eV}$ and $4 \mathrm{eV}$ for both approximations.

\section{- Absorption coefficient}

The absorption coefficient is a valuable parameter of each optoelectronic material. The spectrum of absorption represented in Fig. 2.12 demonstrates that the energy of the threshold, for both the two approximations is around $2.5 \mathrm{eV}$ for $\mathrm{ZnSe}, 3 \mathrm{eV}$ for $\mathrm{ZnO}$, and $1 \mathrm{eV}$ for $Z n S e_{1-x} O_{x}$ with $x$ varies from 0 to 1 . This energy is called the threshold of absorption. Each peak corresponds to an electronic transition, the first transition corresponds to the optical gap. 


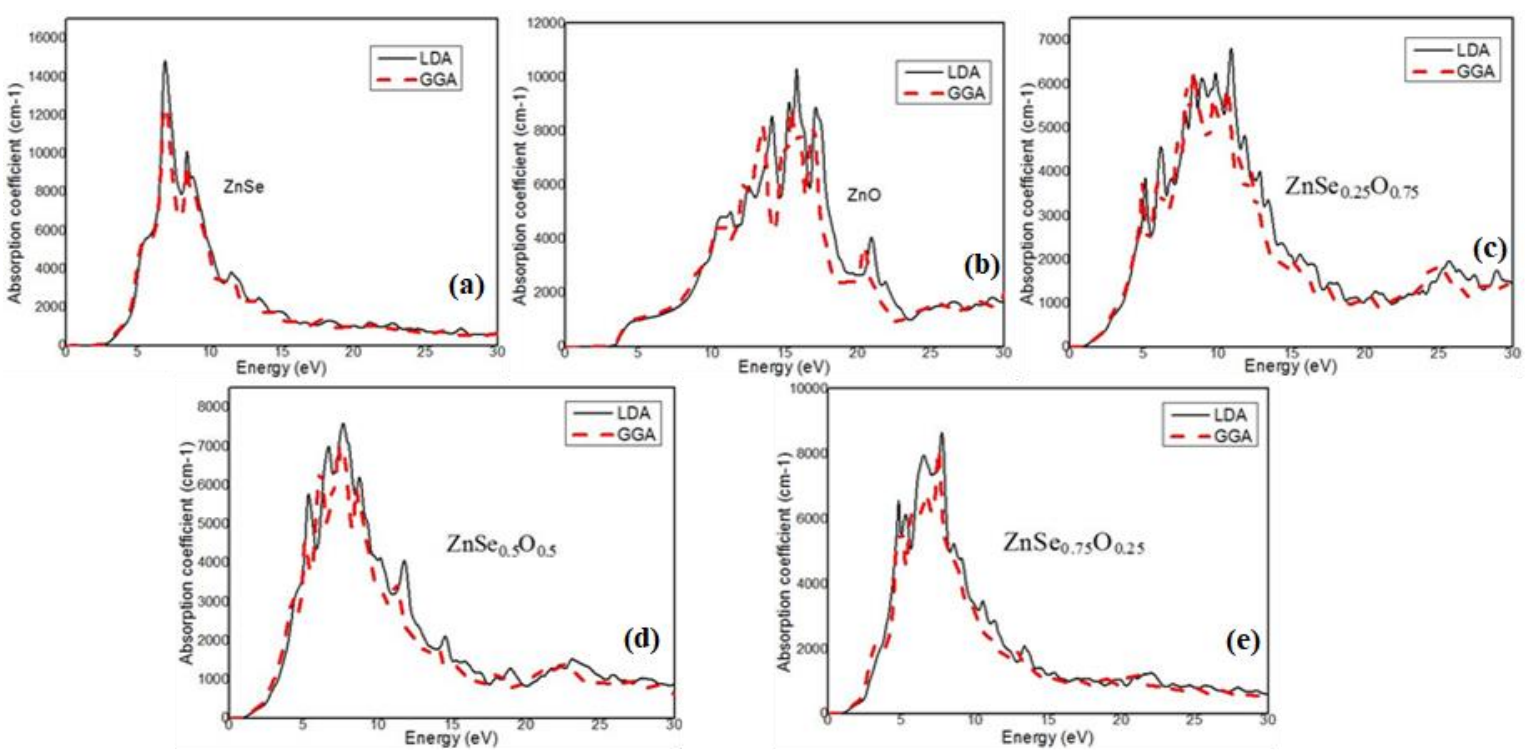

Fig. 2.12: Absorption coefficient according to the energy $Z n S e_{1-x} O_{x}$ for the structure zinc sphalerite with LDA and GGA. (a) $Z n S e$, (b) $Z n O$, (c) $Z n S e_{0.75} O_{0.25}$, (d) $Z n S e_{0.5} O_{0.5}$, (e) $Z n S e_{0.25} O_{0.75}$.

According to the corresponding wavelength of these energies, we can observe that these materials are good candidates to work in the infrared and visible spectra; also, the absorption curve becomes larger according to the oxygen induction; it reaches its maximum in the Ultraviolet field. This leads us to deduce that these components can operate as absorption components of the Ultraviolet waves.

\subsection{Conclusion}

In this chapter a brief presentation of semiconductors has been provided followed by special focus on their optoelectronic properties focusing on semiconductors from group $I I-V I$ which can be used in different optoelectronics devices, especially those applied in optical communication such as LEDs, lasers, photovoltaic cells. We presented the analysis study of the $Z_{n S e_{1-x}} O_{x}$ semiconductor with $(x=0.25,0.5$ and 0.75$)$ proposed group $I I-V I$ using the LDA and GGA approximations in the WIEN2k code. The results obtained are in good agreement with the theoretical results as presented in [Bou 2016]. The obtained simulation results have shown that the variation in oxygen concentration can lead to the production of several optoelectronic devices with different properties that can be useful as robust solid-state lighting such as LEDs and solar cells. 
Chapter 3

\section{Optical Wireless Communications}




\subsection{Introduction}

Nowadays, Optical Wireless Communication (OWC) has become an emerging telecommunication technology complementary to Radio Frequency (RF) and optical fiber communication, which will meet the growing needs of high-speed telecommunication applications and services [Shi 2010]. This technology transmits information through optical radiation in free space, with wavelengths ranging from infrared (IR) [Won 2000] to ultraviolet (UV) [Xu 2008], including the visible light spectrum [Kom 2004]. It allows the transmission of all types of data (data, images and videos) with speeds comparable to that of optical fiber, while having the flexibility and advantages of wireless. This system quickly facilitates deployable, lightweight, high-capacity communication without the need for licensing fees. However, the performance of this system is highly dependent on weather conditions.

The idea of using wireless optical signal as communication channel is not new. In fact, the Photophone invented by Alexander Graham Bell in 1880 [Kil 2002] was the first optical wireless communication device. In his experiment, Bell modulated the solar radiation with a voice signal and transmitted it over a distance. The receiver consisted of a selenium crystal that converted the optical signal into electrical current. He was able to transmit an audible signal over $213 \mathrm{~m}$ [Hay 1988]. This experiment did not work very well due to the crudeness of the employed devices and the intermittent nature of solar radiation. However, the concept remained a curiosity until 1960 when optical sources were discovered, especially the Laser, which announced the real birth of telecommunications systems technology based on optical energy in free space.

In 1979 [Gfe 1979], authors demonstrated the possibility of achieving an indoor communication network of about $100 \mathrm{KBps}$ based on infrared wireless optics. Since that time, the military field and space technology have contributed greatly to the development of research in OWC systems.

In 1990, the IrDA (Infrared Data Association) standard was defined to allow files transfer using infrared spectrum along short distances mainly for remote control of devices. In 1993, new applications were conceived in the field of lighting especially with the development of the diode technology such as the blue and white diode and the wireless optical communication based on visible light (Visible Light Communication, VLC) interested both researchers and industrials.

Visible light communication (VLC) is a wireless optical communication technology where baseband signals are modulated on the light emitted by an LED in the visible spectrum [Nak 2007, Kra 200, Elg 2011, Hra 2013, Tsi 2014, Lou 2014, Lu 2016, Ndj 2018, Kum 2019, Yeh 2019, Lor 2020, Vol 2020]. It is also interesting to note that the Keio University research group was part of the Visible Light Communication Consortium (VLCC) and clearly dominated VLC research until 2007, when other research groups from different parts of the world started to focus their research efforts on VLC.

In Europe, the Fraunhofer Heinrich Hertz Institute of Telecommunications (HHI) is one of the largest research groups of OWC. Their work was based on the development of a high bit-rate OWC system. Their first experimental results were presented in 2008 [Gru, 2008], using a 
receiver based on a PIN photodiode that it was able to obtain a data rate of $40 \mathrm{Mbps}$ using OOK (On Off Keying) modulation and $101 \mathrm{Mbps}$ using DMT (Discrete Multi-tone Modulations). In 2009, they reached a speed of $125 \mathrm{Mbps}$ for a range of $5 \mathrm{~m}$ [Vuc 2009a] using OOK modulation. In the same year, a data rate of $200 \mathrm{Mbps}$ was achieved using DMT [Vuc 2009b]. They also demonstrated in [Vuc 2010] that the data rate can be significantly improved by using an avalanche photodiode instead of a PIN photodiode. In 2010, a joint team of researchers from Siemens and Heinrich Hertz Institute in Berlin were able to transmit at a speed of 500Mbps over a distance of $5 \mathrm{~m}$ [Zyg 2010]. In subsequent years, the data rate continued to increase, reaching 803Mbps [Mob 2012] and 1.25Gbps [Rah 2011]. In July 2011, professor Harald Haas from the University of Edinburgh presented a demonstration related to VLC D-light project, this was the first experimental of VLC transmission system under the acronym of Li-Fi (Light Fidelity) [Haa 2011]. The demonstration showed a High Definition (HD) video being transmitted from a standard LED lamp and the VLC data transmission rate was about $10 \mathrm{Mbps}$, which is the same as those of a DVD.

Furthermore, other several research works have been carried out during the last years both on novel applications and also on the development of new optoelectronic devices for transmitters and receivers in order to increase the transmission rate. In [Han 2008], the authors have presented a submarine VLC transmission using a $1064 \mathrm{~nm}$ LD doubled in frequency and using 1Gbps modulation over a $2 m$ long water pipe. Another research work [Wat 2013] has presented an experimental demonstration of a free space VLC transmission using NRZ-OOK modulation at 2.5Gbps and using a $422 \mathrm{~nm}$ and $\mathrm{LD}$ based on $\mathrm{GaN}$ (gallium nitride). A WDM VLC free space communication system was published by [Chi 2014] where a data rate of 3.75G bps and 2.5Gbps based on SC-FDE (Single-Carrier Frequency-Domain Equalization) and OFDM respectively, was obtained by using an RGB LED (red, green and blue). In [Hua 2015], the authors demonstrated a high-speed VLC system based on a white phosphorescent LED where the OFDM signal is implemented by using the adaptive power bit allocation algorithm. A result, a rate of $2 \mathrm{Gbps}$ and $0.79 \mathrm{Gbps}$ over the distance of $1.5 \mathrm{~m}$ and $3 \mathrm{~m}$, respectively, were achieved. In [Chi 2015], a free space VLC link based on a blue LD GaN

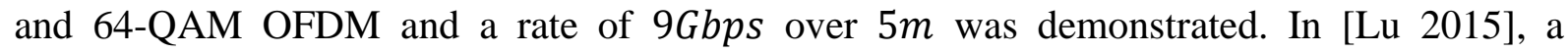
$25 \mathrm{Gbps}$ over $10 \mathrm{~m}$ Li-Fi transmission system based on a $680 \mathrm{~nm}$ VCSEL (Vertical-Cavity Surface-Emitting Laser) transmitter with two-stage locked injection was proposed and demonstrated. A free space VLC transmission system using a $682 \mathrm{~nm}$ VCSEL laser, the OFDM modulation and bit-and-power loading algorithm was presented in [Lu 2016]. A rate of $11.1 \mathrm{Gbps}$ (10.2Gbps raw data rate) was obtained over a distance of $1.2 \mathrm{~m}$ at a bias current of 3.5mA. In [Li 2017], a real-time high-speed VLC transmission system based on RGB LEDs using WDM, was proposed to achieve a transmission rate greater than $500 \mathrm{Mbps}$ in real time, while providing $100 \mathrm{Mbps}$ transmission rate under poor channel conditions. An evaluation of the performance of VLC systems in the presence of solar irradiation at any place and at any time was presented in [Isl 2018], where authors have demonstrated data rates greater than 1 Gbps of a VLC link at high solar luminance measured (i.e. 50350 lux) in clear weather. Furthermore, in [Nlo 2018], the authors proposed a study analysis of a VLC communication system associated in cascade with a hybrid PLC (Power Line Communication) system. 
In this chapter, an overview of the OWC systems will be given, followed by the description of the main optical modulations used for OWC systems. Then, the detail of two systems, namely, the analog and digital wireless optical transmission in free space will be explained. In the first analog OWC system, we are interested in both analogue data transmission and optical wireless energy transmission. However, in the second system we are interested by establishing a transmission protocol to ensure correct data transmission. Finally, the main observations will be summarized in conclusion.

\subsection{Optical Wireless Communication (OWC) systems}

In the literature [Car 2003, Uys 2014, Gha 2016, Qin 2017], Optical wireless communication (OWC) refers to optical transmission in which guided visible light (VL), infrared (IR), or ultraviolet (UV) spectrum are used as propagation channel of communication. Consequently, different kind of OWC systems have been developed for indoor and outdoor applications (Fig.3.1). The Visible Light Communication (VLC), the Li-Fi (Light Fidelity), the Optical Camera Communication (OCC), the Free Space Optical Communication (FSOC) and the optical communication based on Light Detection and Ranging (LiDAR) are the main well known OWC systems. Their classification can be made according to the communication distance [Cho 2018].

Five categories of ranges of the OWC systems are presented in [Cho 2018] and summarized as follow:

- Ultra-short range OWC: In this case, a $\mathrm{nm} / \mathrm{mm}$-level of communications are performed;

- Short range OWC: this communication is often applied in Wireless body area network (WBAN), wireless personal area network (WPAN) and under-water communication;

- Medium range OWC: In this case, for indoor communication range, the VLC-based WLANs (Wireless Local Area Network), Li-Fi and OCC whereas for outdoor communication range V2X communications (vehicle-to-vehicle and vehicle-toinfrastructure connectivity) based on VLC and Li-Fi are often used;

- Long range OWC: This category provides km-range of communication, like interbuilding connections;

- Ultra-long range OWC: This communication range comprises inter-satellite, satellite-earth, satellite-to-airplane, airplane-to-satellite, airplane-to-airplane, and airplane-to-ground links. 


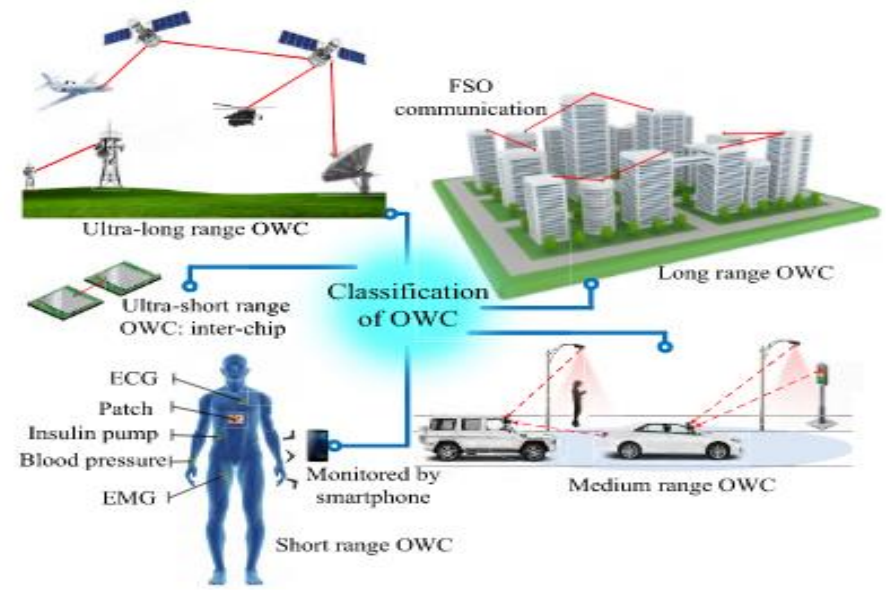

Fig. 3.1: Examples of OWC for different ranges of communications [Cho 2018].

In table 3.1, the main advantages and limitation of the OWC systems are summarized.

Table 3.1: Advantages and limitation of OWC.

\begin{tabular}{|c|c|}
\hline Advantages of OWC & Limitation of OWC \\
\hline $\begin{array}{l}\text { - License-free operation; } \\
\text { - Huge unregulated bandwidth; } \\
\text { High level of security (because light signals } \\
\text { cannot pass through walls and can therefore } \\
\text { be conned within a well-defined coverage } \\
\text { zone); } \\
\text { - Low power consumption; } \\
\text { - Low cost; } \\
\text { - No interference with electronic equipment } \\
\text { and other communication networks; } \\
\text { - Very high achievable SNR; } \\
\text { Easy integration into the existing lighting } \\
\text { arrangement. }\end{array}$ & $\begin{array}{l}\text { - } \quad \text { Obstacles block transmission channel; } \\
\text { - } \quad \text { Sensitive to sudden blocking a connection; } \\
\text { - } \quad \text { Limited transmitted power }\end{array}$ \\
\hline
\end{tabular}

\subsubsection{OWC systems design}

A standard OWC system (Fig. 3.2) consists of a light emitting subsystem (emitter) and a light detection subsystem (receiver) and free space is the medium for the optical signal propagation. 


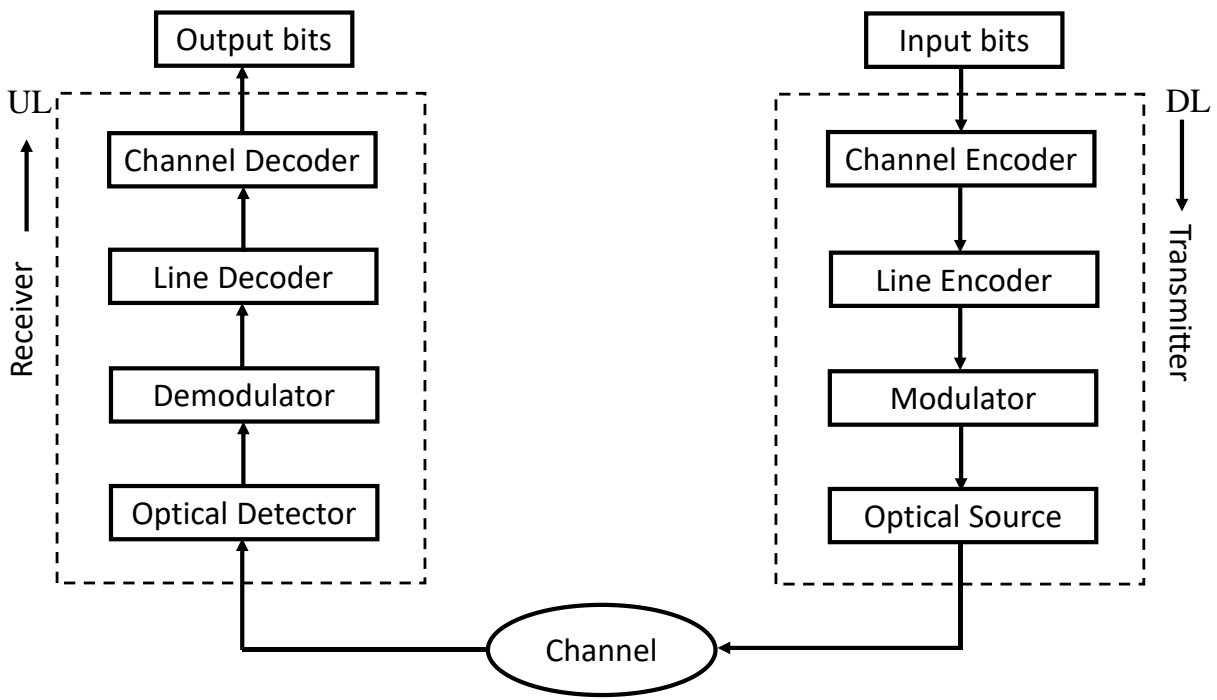

Fig. 3.2: Basic architectures of OWC systems.

In the case of digital transmission, the input data which are electrical signals are encoded (channel and line encoders), then modulated and converted into an optical signal transmitted by an optical source transmitter.

In the reception part, the optical detector receives through the free space the optical signal and transforms it into electrical current, then demodulates and decodes it to obtain levels of electrical current for proper digital signal recovery. However, it should be noticed that the receiver based on direct detection only detects the intensity of the optical wave, without frequency or phase information.

Regarding light sources used in OWC systems, there are different alternatives. The most popular are Laser Diodes (LD) and LEDs. For detection, the photodiodes (PDs) or photodetectors oftenly used are the p-i-n junction (PIN) and avalanche photodiodes (APDs) [Kah 1997, Gha 2012]. However, photovoltaic cells [Wan 2015, Lor 2020] and cameras [Zha 2018, Eso 2019] are also used as low speed optical detectors.

\subsubsection{Configuration of OWC links}

One of the main challenges to be carefully considered during the design and modeling stages of an OWC system is the localization status of the transmitter - receiver pair which mainly defines how the signal is transmitted. Design of an OWC link is essentially based on two characteristics as depicted in Fig. 3.3:

a) The Line-of-Sight (LOS). In this case, the communication is direct and established without any interruption of signals between transmitter and receiver. The directivity of signals between transmitter and receiver can be classified into three classes: direct, hybrid or non-direct, as shown in Fig.3.3. Directed link (in Fig. 3.3.a) design maximizes power efficiency, since it minimizes path loss and reception of ambient light noise. On the other hand, non-directed links (in Fig. 3.3.c) may be more convenient to use, particularly for mobile terminals, since they do not require aiming 
of the transmitter or receiver. It is also possible to establish hybrid links (in Fig. 3.3.b), which combine transmitters and receivers having different degrees of directionality [Kah 1997].

b) The Non-Line-of-Sight (NLOS). Here, the communication is not direct between transmitter and receiver as depicted in Fig. 3.3.d), Fig. 3.3.e) and Fig. 3.3.f), rely upon reflection of the light from ceiling or some other diffusely. These classifications are based on the radiation pattern of the transmitter and field of view (FOV) of the receiver.

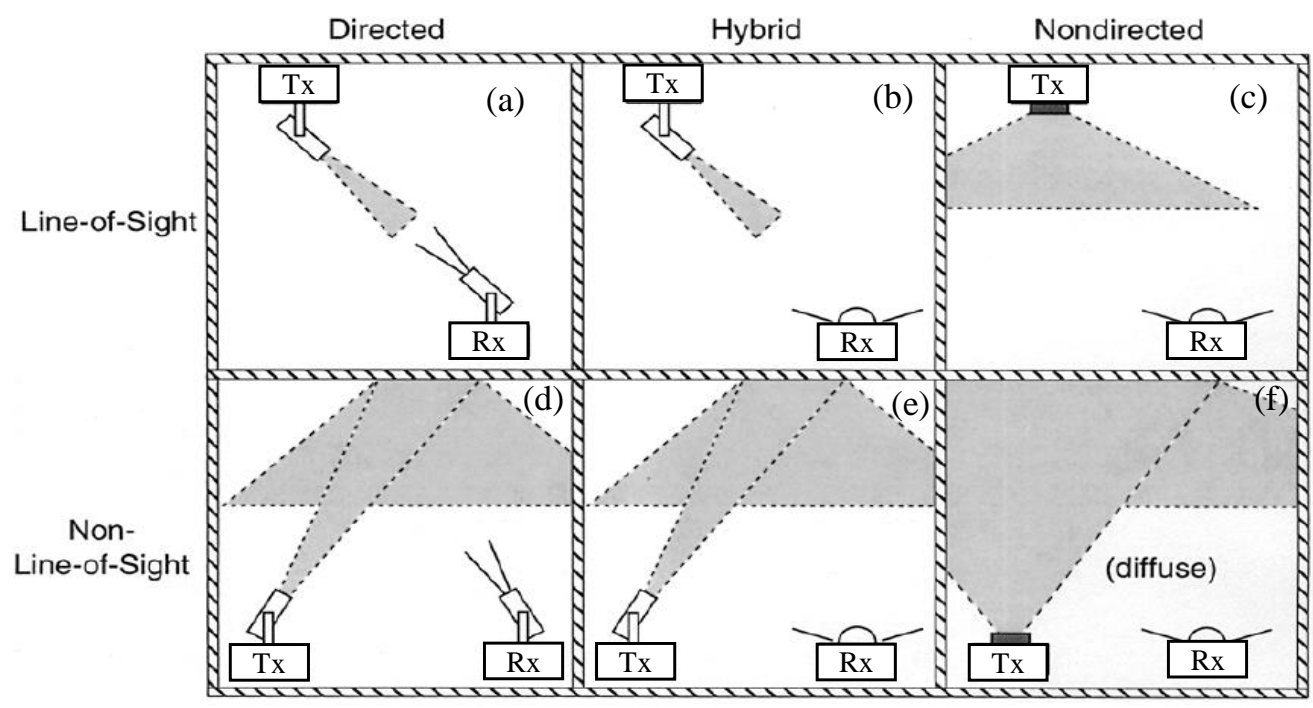

Fig. 3.3: Classification of Indoor OWC communication links [Kah 1997].

The LOS links maximize energy efficiency and transmission speeds, while NLOS is robust and useful. As shown in Fig. 3.3, the non-directed links, known also as diffuse links, offer the greatest robustness and mobility while maintaining connectivity [Zha 2018, Yon 2019].

In the design of wireless optical communication systems especially based on visible light, a LOS configuration between the transmitter and receiver is recommended [Poo 2015, Zha 2018, Ndj 2018, Yon 2019, Kum 2019, Vol 2020].

The OWC systems are based on the same design and working principle (Fig. 3.2). However, they may differ in terms of modulation technique, transmission system, reception system and the channel of transmission. For transmission, LEDs or LDs are mainly used as transmitters. Photodetectors or cameras are used as receivers. The communication channel can be infrared (IR), visible light (VL) or ultraviolet (UV) spectrum (see Fig. 3.4). The following table explains how the different OWC technologies differ from each other in terms of transmitter, receiver and the channel of communication for Uplink (UL) and Downlink (DL) communication. 
Table 3.2: COMPARISON of different OWC systems.

\begin{tabular}{|c|c|c|c|}
\hline Issue & $\mathrm{Li}-\mathrm{Fi}$ & VLC & FSO \\
\hline Transmitter & $\begin{array}{l}\text { LED/LD (combined LDs } \\
\text { with optical diffuser) }\end{array}$ & LED/ LD & LD \\
\hline Receiver & PD & PD/ Camera & PD \\
\hline Spectrum & IR/ VL/ UV & VL & IR/VL/UV \\
\hline Path loss & $\begin{array}{l}\text { Medium (very high for } \\
\text { NLOS) }\end{array}$ & $\begin{array}{l}\text { Medium (very high for } \\
\text { NLOS) }\end{array}$ & High \\
\hline Main purpose & $\begin{array}{l}\text { Illumination and } \\
\text { Communication }\end{array}$ & $\begin{array}{l}\text { Communication, } \\
\text { illumination } \\
\text { localization }\end{array}$ & Communication \\
\hline Main limitations & $\begin{array}{l}\text { (i) Short distance } \\
\text { communication, and (ii) } \\
\text { not suitable in outdoor }\end{array}$ & $\begin{array}{l}\text { (i) Short distance } \\
\text { communication, (ii) no } \\
\text { guaranteed of mobility } \\
\text { support, and (iii) not } \\
\text { suitable in outdoor }\end{array}$ & $\begin{array}{l}\text { Environment } \\
\text { dependent }\end{array}$ \\
\hline
\end{tabular}

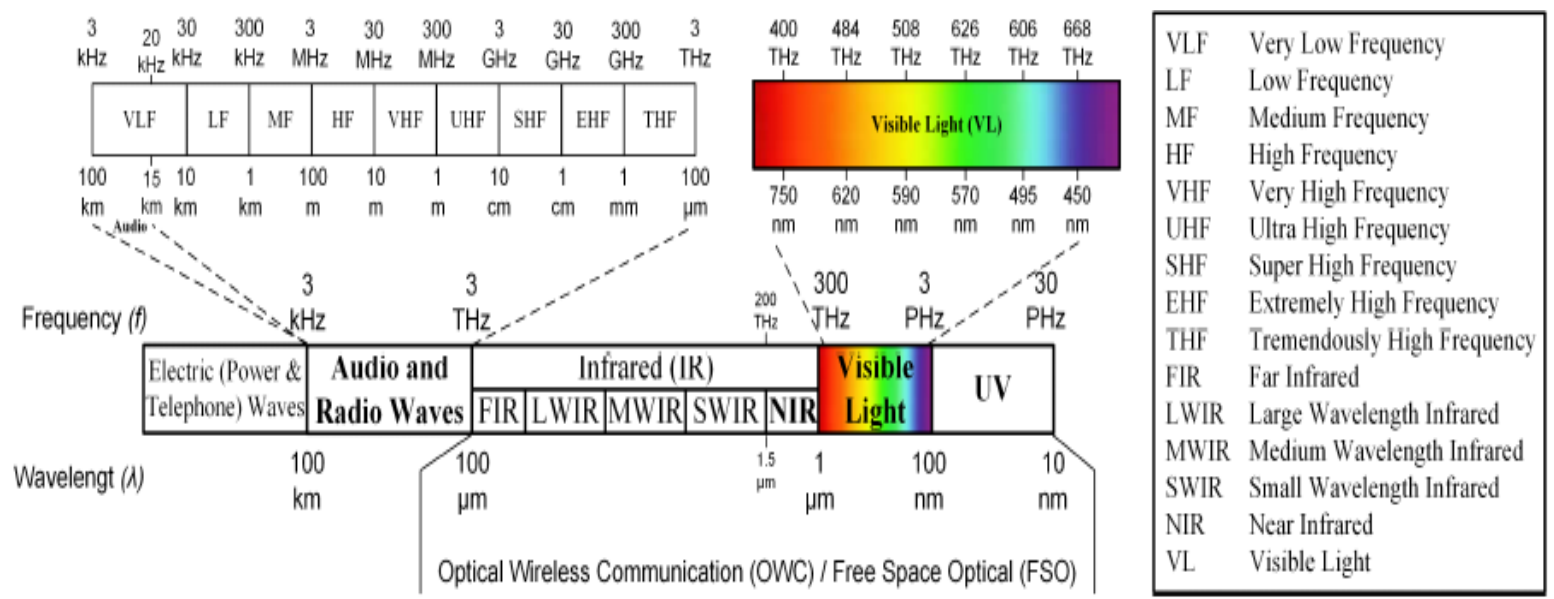

Fig. 3.4: Part of the electromagnetic (EM) spectrum showing the frequency and wavelength ranges for each band.

\subsubsection{Types of OWC systems}

\subsubsection{Visible Light Communication (VLC)}

Visible Light Communication (VLC) is an emerging and promising technology. The VLC technology is based on the use of the spectrum of visible light [380 $\mathrm{nm}-780 \mathrm{~nm}$ ] as a means of communication as shown in Fig. 3.4. It offers huge bandwidths with free license; it isn't harmful and allows high speed transmission. The LEDs or LDs are used generally for transmission combining both lighting and communication. The VLC allows omnipresent communication and addresses the gaps and limitations of RF communication. Different research works were focused on development of this new promising communication such as [Mmb 2016, Yon 2019, Kum 2019, Gao 2019, Li 2019a, Yeh 2019, Zho 2019]. However, this technology suffers from a few limitations such as its effectiveness for outdoor applications and long-distance communications. 
Other VLC applications for indoor and outdoor environments summarized in [Kha 2017b] [Zhu 2017] have become important during the last years since VLC is an attractive solution for V2X communications, smart displaying signboards, underwater communications, internet in Hospital, internet in planes, ... etc. Actually Li-Fi is the subset of communication systems which employ visible light both for illumination and communications. Its applications and limitations are similar to those of VLC.

\subsubsection{2. $\quad$ Free Space Optical Communication (FSO)}

Free Space Optical Communication (FSO) is operating using the Near IR (NIR), VL and UV spectrum as communication medium. This technology often uses LDs rather than LEDs for the transmission where the illumination is not required. Narrow beams of focused light from an LD transmitter are used to establish high-data-rate communication links between two fixed points over distances ranging from a few nm to several thousand kilometers [Kha 2014, Mal 2015, Kum 2015, Fan 2018, Li 2019b].

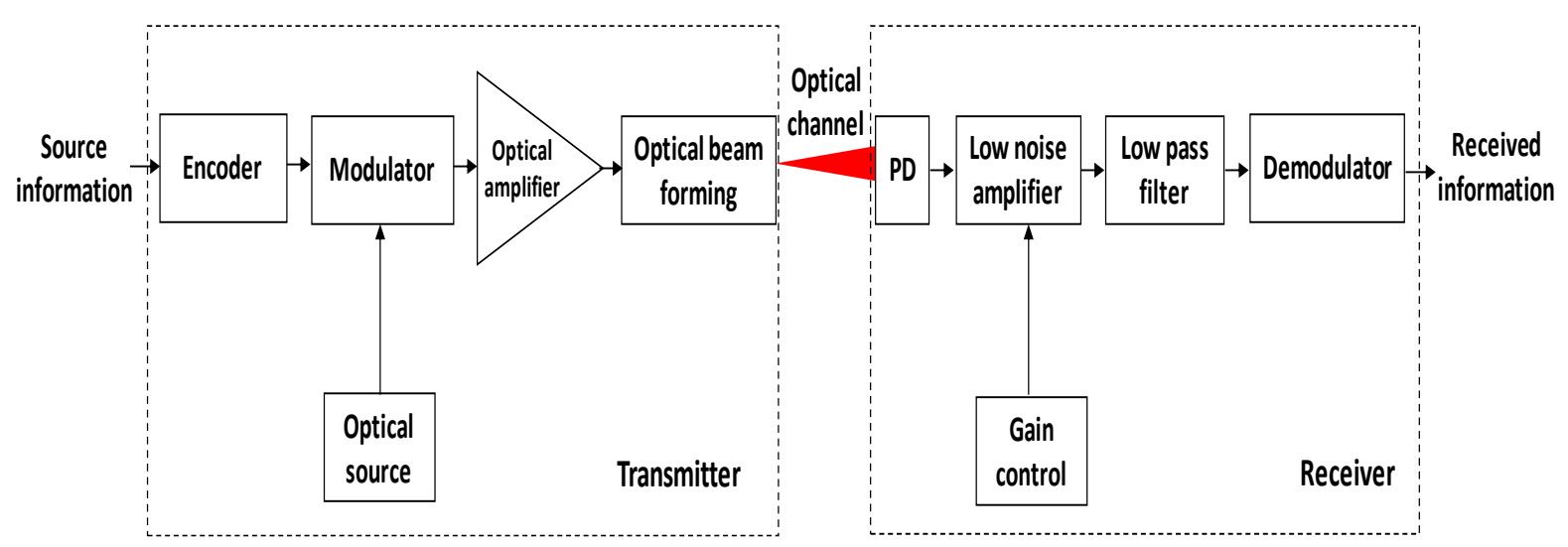

Fig. 3.5: Basic block diagram of an FSO system [Kha 2014].

In Fig. 3.5, the basic block diagram of an FSO system is presented where a source information data input is firstly encoded using channel and/or line encoding before modulation. If required, an optical amplifier can be used to increase the power intensity of the modulated laser beam. Then, the light beam is collected and refocused by means of beamforming optics before being transmitted over long distance [Kha 2014].

The optical source used in FSO systems should deliver a comparatively high optical power over a wide temperature range. Moreover, it should have a long mean time between failures (MTBF) and the corresponding components should be small in footprint and have low power consumption [Kha 2014]. The important features of the optical transmitter in FSO systems are size, power, and beam quality, which determine the laser intensity and minimum divergence obtainable from the system [Kha 2014]. The receiver front-end comprises optical filters with a lens to collect and focus the received beam on the PD. The PD coverts the received optical signal to current and/or voltage. The low-pass filter is used to limit the thermal and 
background noise levels. Finally, the demodulator performs the necessary demodulation process to recover the original data.

The FSO links have very high available bandwidth, thus providing considerably higher data rates. However, despite many advantages of FSO communication across a wide range of applications [Kha 2014], it suffers from link reliability, especially in long-range communications, owing to its high sensitivity to several factors. These include weather conditions (e.g., fog, rain, haze, sandstorms, clouds, snow, and scintillation can increase attenuation), atmospheric turbulence (causing fluctuations in the density of air, leading to a change in the air refractive index), and physical obstructions (e.g., flying birds, trees, and tall buildings can temporarily block the signal beam) [Mal 2015].

\subsubsection{Optical modulation for OWC systems}

\subsubsection{Factors affecting modulation techniques for OWC systems}

Several modulation techniques were associated with optical wireless communications depending on their indoor or outdoor environments, link configurations as well as the equipment deployed in the OWC systems [Cho 2010, Shi 2010, Gha 2013].

For outdoor environment, the main problems are heavy fog, strong turbulence and eye safety. To deal with these problems, a tradeoff should be made in order to pump more optical power or concentrate and focus more power into smaller areas with a limitation on the amount of optical power for eye safety.

In indoor environment, a strict limitation for the transmitted optical power is established for the eye safety. In this case, two factors are to be considered (a) dimming and (b) flickering: In fact, different activities require different illuminances, such as 30-100 lux that is required for normal visual activities in public locations. In general, the relation between the perceived light and the measured light can be formulated as follow [IES 2000]:

$$
\text { Perceived Light }(\%)=100 * \sqrt{\frac{\text { Measured Light (\%) }}{100}}
$$

The changes in brightness of the modulated light should be done in a way that it should not result in human-perceivable fluctuations. According to IEEE 802.15.7, the switching should be done at a rate faster than $200 \mathrm{~Hz}$ for avoiding harmful effects.

On other hand, additional criteria are made in the selection of the modulation technique such as:

- Transmission reliability: a modulation technique must be able of providing an acceptable error rate under unfavorable conditions, as well as resistance to multipath interference and variations in the DC component of the data signal;

- Adaptation to the flickering effect: for too low flow rates or too high differences between symbol levels, the human eye is likely to perceive a flickering phenomenon of light, which can be very unpleasant; 
- Bandwidth efficiency: Although optical wireless communications systems have a large bandwidth, it can be limited by various parameters such as the bandwidth of the transmitter/receiver components or multipath optical beam interference.

- Energy efficiency: In order to comply with eye and skin safety regulations, the average optical power emitted by a transmitter to a wireless optical receiver is limited [Kah 1997]. In addition, in battery-powered portable equipment, it is desirable to minimize electrical energy consumption, which also imposes limits on the optical transmission power. Therefore, the most important criterion for evaluating modulation techniques suitable for indoor optical wireless communication systems (VLC) is energy efficiency;

- Other factors: such as the cost of the equipment used maintenance, and complexity are also to be considered.

\subsubsection{Types of modulation techniques used in OWC systems}

Modulation in OWC is achieved by using variations in the intensity of the light corresponding to the information in the message signal. Different techniques have been developed depending on the configuration links of the OWC systems, the communication protocols, the equipment and the desired transmission rate.

In the following, we will summarize the most important modulation techniques used in OWC systems.

\section{a) Analog Intensity Modulation}

In most practical OWC systems, the intensity modulation/direct detection (IM/DD) scheme is widely deployed for indoor and outdoor applications due to its simplicity and low cost compared to the digital modulation scheme.

In the IM/DD, the intensity of the light source is modulated with an analogue signal (or premodulated RF signal) and a Direct Detection (DD) is accomplished based on a photodetector to recover the analogue signal.

The limited bandwidth of the optical sources as well as their nonlinear power to-current characteristics don't allow to employ the simplest scheme of IM/DD modulation for high rate transmission (more than a few Gbps). To overpass this limitation in IR transmission systems, different researchers resort to an external modulator (such as the Mach-Zehnder Modulator (MZM)) to modulate the intensity of the continuous optical wave (in most cases a laser beam), together with a coherent-based $R_{x}$ to recover the baseband signal [Chi 2017b, Sek 2018]. 


\section{b) Digital Optical Modulations}

\section{- On-Off Keying (OOK)}

The OOK modulation is a baseband modulation and one of the simplest modulations in the field of optical communications, especially for IM/DD systems. This technique is composed of two symbols ( 0 or 1$)$. Level "1" corresponds to the on state, while level "0" corresponds to the off state. In OOK, the LED is not turned completely off in the off state, but the reduction in the level of intensity is performed. The main advantage of using OOK is its easy implementation. The OOK modulation (Fig. 3.6) can use the codes Non-Return-to-Zero (NRZ), Return-to-Zero (RZ) or Manchester. A data rate of 10Mbps was demonstrated in [Par 2007] using NRZ-OOK with a white LED. OOK is a suitable for OWC modulation approach and is easy to implement in such systems due to the ability of LDs and LEDs to switch on and off quickly. However, OOK is not efficient when compared with other modulation formats [Wan 2017].

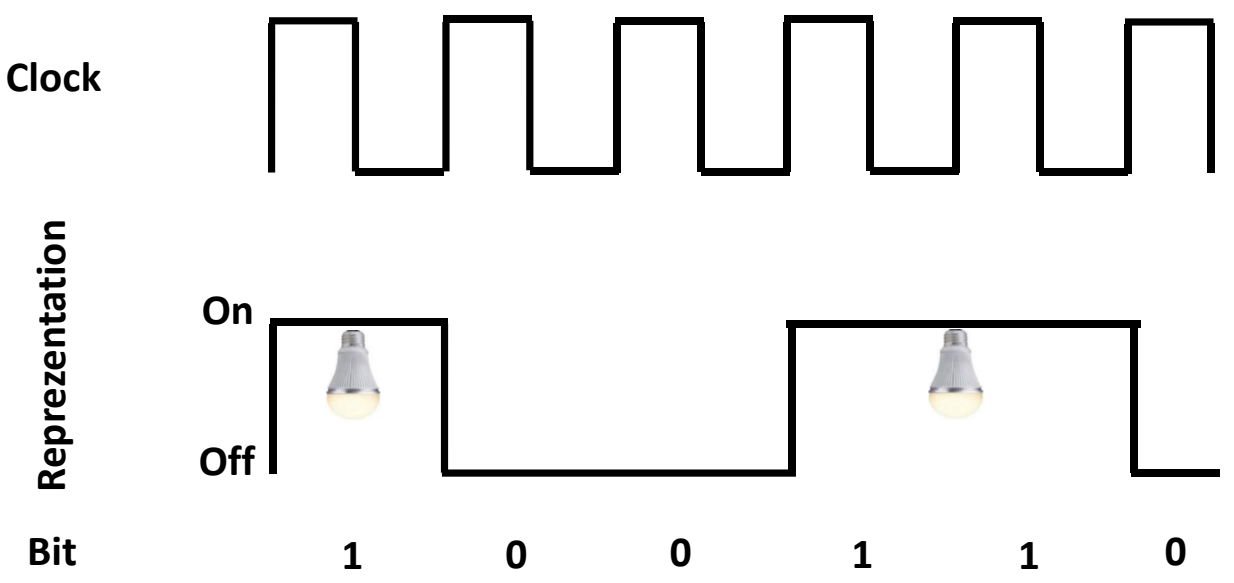

Fig. 3.6: On - Off Keying data coding.

\section{- Pulse modulation techniques}

\section{$\checkmark$ Pulse Amplitude Modulation (PAM)}

Despite the simplicity of OOK modulation, it remains limited by the low data rate it can achieve. Therefore, researchers were motivated to develop new modulation techniques with higher data rates. PAM modulation is another simple modulation technique that is widely considered in OWC systems. However, OOK modulation is a type of PAM with only two levels [Gha 2017, Kha 2017a, Bac 2019].

PAM is a very basic modulation scheme which is bandwidth efficient. Data is modulated into the amplitude of the signal pulse. This type of modulation is similar to OOK: the main difference with OOK is that the carrier signal has two levels, whereas with PAM, the carrier signal will have 4 or more levels (Fig. 3.7), depending on the modulation order. M-PAM is another notation to indicate it, and $\mathrm{M}$ represents the modulation order. In the case of M-PAM 
each symbol has $M$ bits, therefore, the symbol error rate would be the error associated with the symbols.
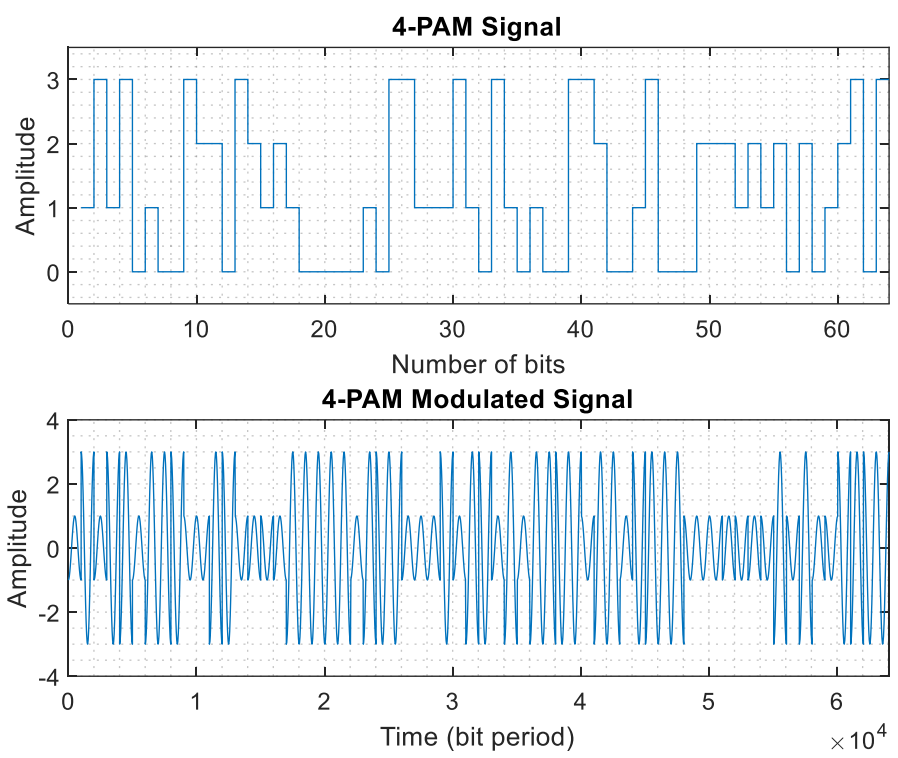

Fig. 3.7: 4-PAM Modulation.

Modulation schemes employing multiple intensity levels such as PAM may undergo nonlinearity in LEDs luminous efficacy. Due to the dependence of the color of LED emission on input current and temperature, multiple symbol levels of PAM are subject to shifts in color temperature due to variation in drive current.

\section{$\checkmark$ Pulse Position Modulation (PPM)}

A Pulse position modulation (PPM) [Pat 2015] is based on the position of the pulse. It improves OOK's energy efficiency, but at the cost of increased bandwidth requirements and complexity. In the PPM, the duration of the symbol is divided into equal intervals denoted by $t$ slots where the transmission of the pulse takes place in any one of the slots [Buh 2014, Far 2019]. Since only one pulse in each symbol period is present in the case of PPM, the problem of low data rate still arises when using this modulation.

Multi-pulse PPM (MPPM) [You 2015, Sha 2020] was introduced by making the PPM more spectrally efficient using the transmission of multiple pulses in each symbol-time. Expurgated PPM (EPPM) is the modified version of the PPM that was introduced to improve the performance of peak-power limited $M-\operatorname{ary}$ communication systems [Nos 2012]. Multilevel EPPM (MEPPM) was introduced in [Nos 2012] for the spectral effectiveness of the PPM because the spectral efficiency of the MPPM and EPPM was less than 1.

VPPM modulation is a variant of Pulse Position Modulation (PPM) developed specifically for optical wireless communications in VLC free space [Lee 2011]. VPPM modulation provides a variable range and constant data rate (Fig.3.8). This is achieved by adjusting the pulse width. 
The advantage of this type of modulation is that it protects against intra-frame flicker since the pulse amplitude is maintained constant.

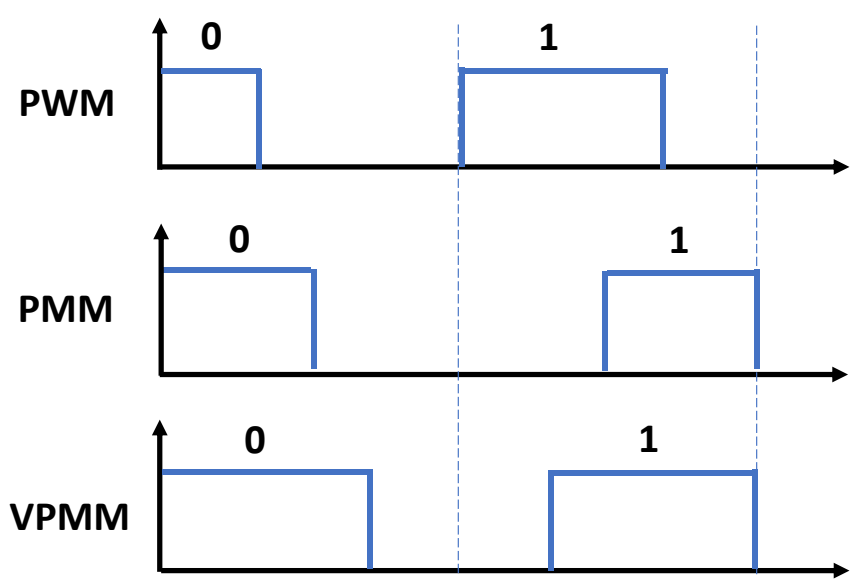

Fig. 3.8: Schematic diagram showing the difference between Pulse Width Modulation (PWM), Pulse Position Modulation (PPM), Variable Pulse Position Modulation (VPPM).

\section{- Color Shift Keying (CSK)}

CSK modulation is specific to VLCs, was proposed in IEEE 802.15.7 to enhance the data rate which was low in other modulation schemes. Modulation in CSK is performed using the intensity of the three colors in an RGB LED source. Unlike other modulations, the symbols of the CSK modulation are selected so that the light output remains constant while the different color components are varied. It maps all colors perceivable by eye to two chromaticity parameters.

\section{- Multicarrier modulation}

More advanced techniques can be used in OWC systems to transmit multiple carriers, such as subcarrier modulation (SCM). This can provide multiple access for simultaneous users and a high data rate. However, SCM is not power efficient like single carrier schemes [Car 1994]. In [Wan 2017], Optical orthogonal frequency division multiplexing (OFDM) was applied in VLC systems to achieve high data rates over a noisy channel and to reduce inter-symbol interference (ISI). The system, however, did not achieve a high SNR. This modulation technique will be more detailed in chapter 4 .

In general, the use of complex modulation leads to an improvement in the performance of the OWC systems, such as mitigating the ISI effect and increasing the data rates. However, these modulation techniques require a complex transceiver. 


\subsection{Design of Experimental prototypes}

Different prototypes were designed for both analog and digital optical wireless communication in indoor environment. Mainly, the transmission modes were simplex and half duplex. The LEDs are used as emitter where different receivers were tested.

However, before starting the design of the transmission system, the format of the signal to be transmitted is the keynote of the practical design of different prototypes.

In the following, we will transmit audio, texts and images signals. In fact, optical wireless communication opens the possibility to combine illumination, optical wireless communication and optical wireless power transfer.

\subsubsection{Analog wireless transmission using visible light}

Many authors were focused on developing different prototypes for audio transmission [Ruf 2015, Rey 2017]. In this section, our interest is not limited to design an analog optical wireless communication for audio transmission, but we plan to design a system that will be able simultaneously to illuminate, transmit audio signal using optical wireless communication and accomplish a wireless energy transfer.

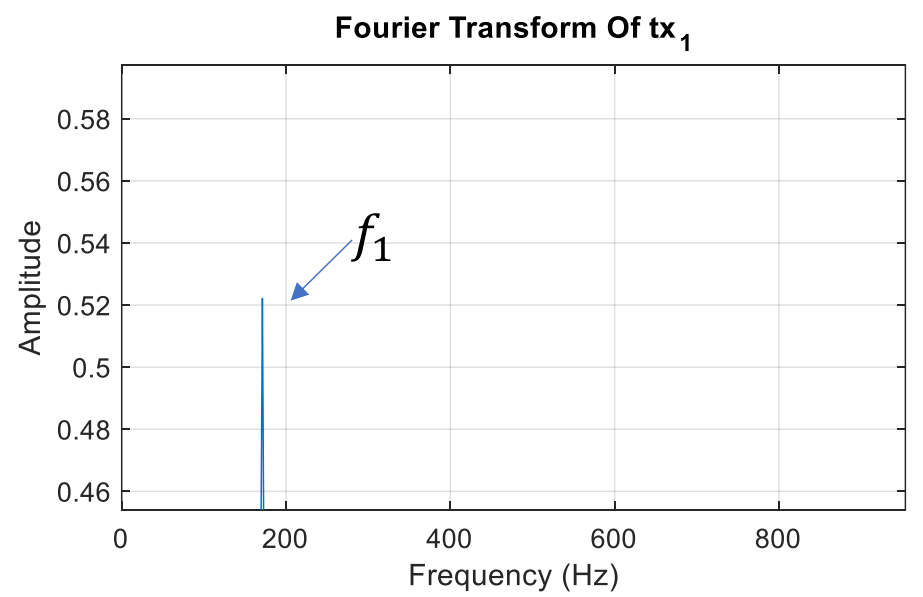

Fig. 3.9: The frequency spectrum of our transmitted audio signal.

In the following, we will consider an audio signal, which is a complex acoustic signal composed of several frequencies. It can be pure or complex. An audio sound has a fluctuating evolution over time. It is characterized by the following parameters [Dar 2017]:

- Amplitude: this is one of the main characteristics of a sound. The perceived strength depends, among other things, on the amplitude. The sound can be strong or soft. In air, amplitude corresponds to the pressure variations of the wave. On a frequency spectrum, the amplitude of a sound is therefore determined by the frequency $f_{1}$ of the first peak (Fig.3.9);

- Intensity: the intensity of a sound distinguishes a strong sound from a weak sound. This is commonly referred to as volume. The greater the amplitude of the sound 
vibrations emitted, the louder the intensity of the sound emitted and the higher the peaks in the frequency spectrum;

- Bandwidth: for an audio signal, it is related to that of the human ear which is between $20 \mathrm{~Hz}-20 \mathrm{KHz}$. This bandwidth can be modified by electronic equipment or even the speaker (depending on whether of the sound is higher or lower, e.g. a young person's hearing is not the same as an old person's);

- Dynamics: this is the difference between the loudest level and the background noise, often expressed in $d B S P L$ (decibel Sound Presure Level). For example, the human ear has a dynamic range of $120 \mathrm{dBSPL}$ since the smallest sound a person can hear is $0 d B S P L$ and above $130 d B S P L$ the sound is painful and dangerous. Dynamic range is also used to characterize audio material (the sound level felt by the human ear).

\subsubsection{System description}

In this part, we will present a simple and an efficient system for analogue transmission of an audio signal in free space. This system has the ability of illumination, optical wireless communication using visible light and wireless power transfer in order to recover and recycle the received energy.

Firstly, the observation of the audio signal (Fig. 3.10) demonstrates that this signal is fluctuating and composed both on negative and positive information. However, the emitter (LED) cannot support negative signal values.

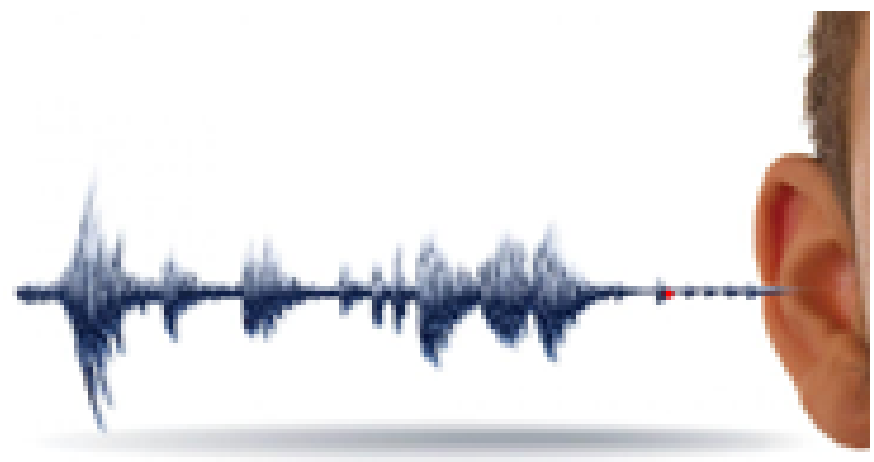

Fig. 3.10: Time presentation of the audio signal.

The most straightforward approach to generate a positive signal from a real signal is to impose a proper DC bias. Hence, in the proposed prototype (Fig. 3.11), a DC Bias will be applied to the audio signal. Then, Intensity Modulation (IM) is applied to the electrical signal through an emitting LED. The DC Bias is chosen such that the mean value of the positive signal lies on the center point of the linear range of the optical emitter. 


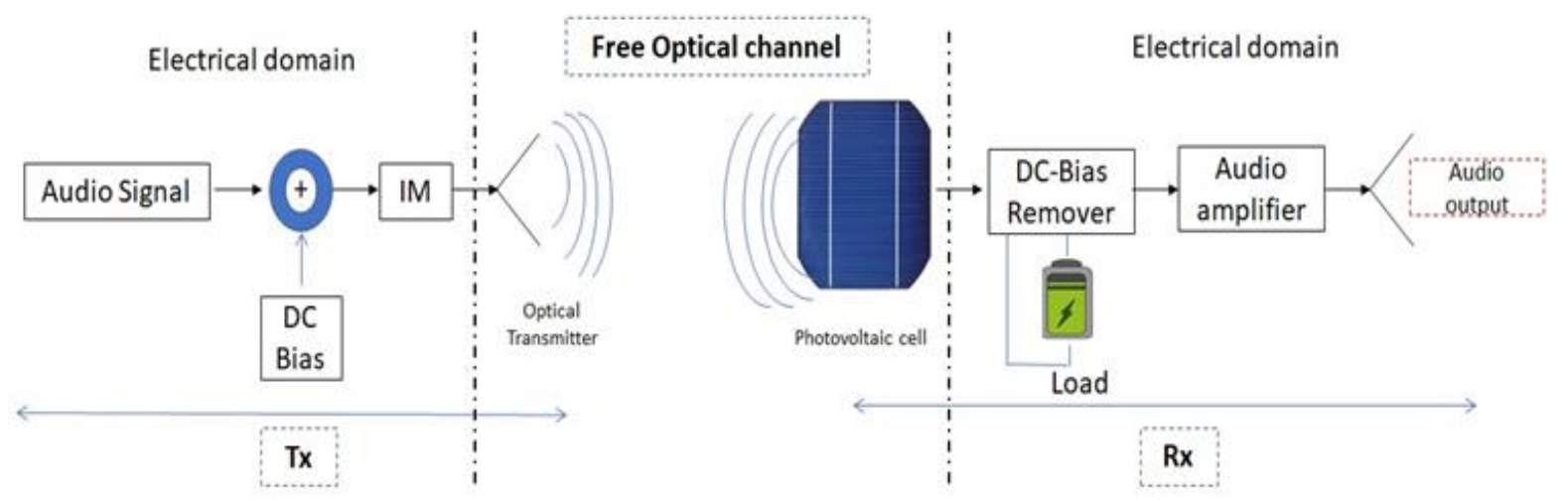

Fig. 3.11: Block diagram of simultaneous Optical Wireless audio transmission and energy harvesting.

The LED output signal (i.e. optical waves) is launched into the free space carrying audio data. In the reception part, a photovoltaic cell ensures the conversion of optical energy into electrical energy. However, the received signal fades away often, especially if the distance between the transmitter and the receiver is large; consequently, an amplifier is added to recover and upgrade the received signal (Fig. 3.11). Then, the DC component is removed and stored in a rechargeable battery. The audio signal is then sent to the audio output port.

\subsection{Experimentation and results}

\subsubsection{Analog wireless transmission using visible light}

The prototype of the indoor analog audio wireless transmission using visible light is organized as follow:

Source: is an audio output from the jack socket of a computer or mobile phone;

Transmitter block is composed of the audio source, followed by the TDA7297 $15 w$ Dual Channel AC/DC 12V - Digital audio amplifier and a white LED (3W High Power Led) used as emitter and acting in a range of values of $3.2 \mathrm{~V}-9 \mathrm{~V}$;

> Receiver block: in this part, a photovoltaic cell with reference JZ - PV11570 and dimension $(11.5 \mathrm{~cm} \times 7 \mathrm{~cm} \times 0.2 \mathrm{~cm})$ receives the optical signal, demodulates the light intensity and transforms it into an electrical signal. Then, an audio amplifier (LM 385) is used for level adaptation followed by a DC-bias remover subsystem. The DC- Bias is then charged in a battery using a mini Solar Lipo charger with reference CN3065. The remaining audio signal is then send to the loudspeaker, respecting the adaptation threshold for amplification. 
Table 3.3: Specifications of the equipment in the Analog wireless transmission using visible light system.

\begin{tabular}{|c|c|c|}
\hline & Model & Characteristics \\
\hline LED & White LED & $\begin{array}{l}3 W \text { high power } \\
220 \mathrm{Lm}\end{array}$ \\
\hline Cellule PV & JZ-PV11570 & $\begin{array}{l}\text { Power range: } 0.36 W p \\
\text { Voltage et maximum power } \\
(V m p p): 6 \mathrm{~V}\end{array}$ \\
\hline Audio amplifier & LM 385 & $\begin{array}{l}\text { Adjustable from } 1.24 \mathrm{~V} \text { to } \\
5.30 \mathrm{~V} \\
\text { Operating Current of } 10 \mu \mathrm{A} \text { to } \\
20 \mathrm{~mA} \\
\text { Maximum gain value: } 200\end{array}$ \\
\hline $\begin{array}{l}\text { Mini Solar Lipo } \\
\text { charger board }\end{array}$ & CN3065 & $\begin{array}{l}\text { Input voltage : } 4.4 \mathrm{~V}-6 \mathrm{~V} \\
\text { Output voltage/ current : } \\
5 \mathrm{~V} 500 \mathrm{~mA}\end{array}$ \\
\hline Digital audio amplifier & TDA7297 & $\begin{array}{l}15 w \text { Dual Channel AC/DC } \\
\text { Operating Voltage: } 6 \mathrm{~V} \text { to } 18 \mathrm{~V} \\
\text { Peak Output Current: } 2 \mathrm{~A} \\
\text { Closed Loop Voltage gain: } \\
33 d B\end{array}$ \\
\hline
\end{tabular}

The audio signal recorded as MP3 audio file and presented in Fig. 3.12 shows the audio signal in time with negative and positive values with maximum peak to peak voltage of $2.74 \mathrm{~V}$ and frequency around $180 \mathrm{~Hz}$.

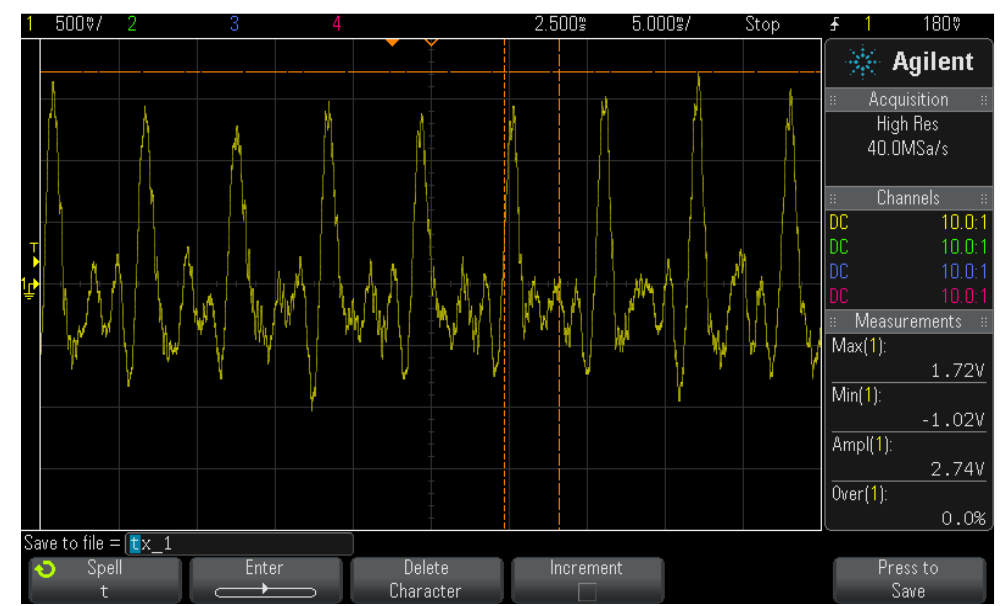

Fig. 3.12: The audio signal to be transmitted. Time is represented in the $x$-axis.

Since LEDs are diodes, they must be DC powered and forward biased to emit light. Generally, the direct voltages determine the size of the LED to be used; the smallest LEDs operate at very low continuous voltage $(1.5 \mathrm{~V}$ to $3.3 \mathrm{~V})$. There are also LEDs that can be powered from $5,12,24$ volts or more. It is necessary to add a protection resistor, which value is chosen so that the minimum voltage is reached for the LED to emit light. 


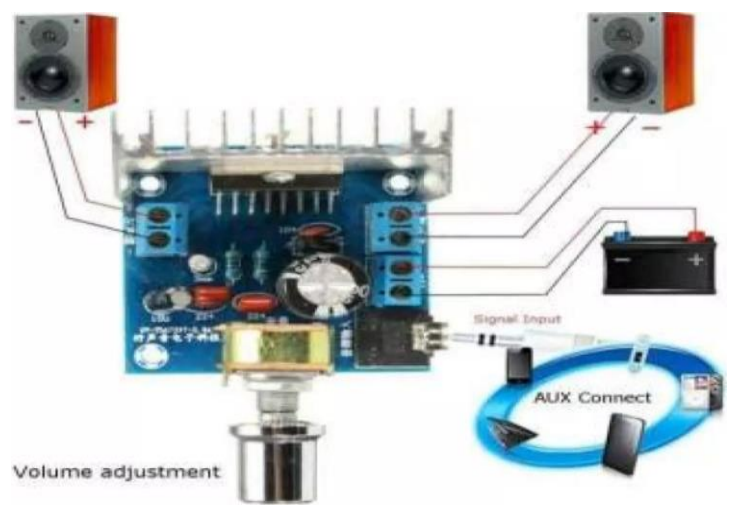

Fig. 3.13: TDA7297 15w Dual Channel AC/DC 12V -Digital audio amplifier.

To add up our voltages AC and DC, different systems can be used. In our experiment, we have tested different systems and we opted to the choice of the TDA7297 15w Dual Channel AC/DC $12 \mathrm{~V}$-Digital audio amplifier presented in (Fig. 3.13). This system has the ability to amplify audio signal and to add a DC bias.

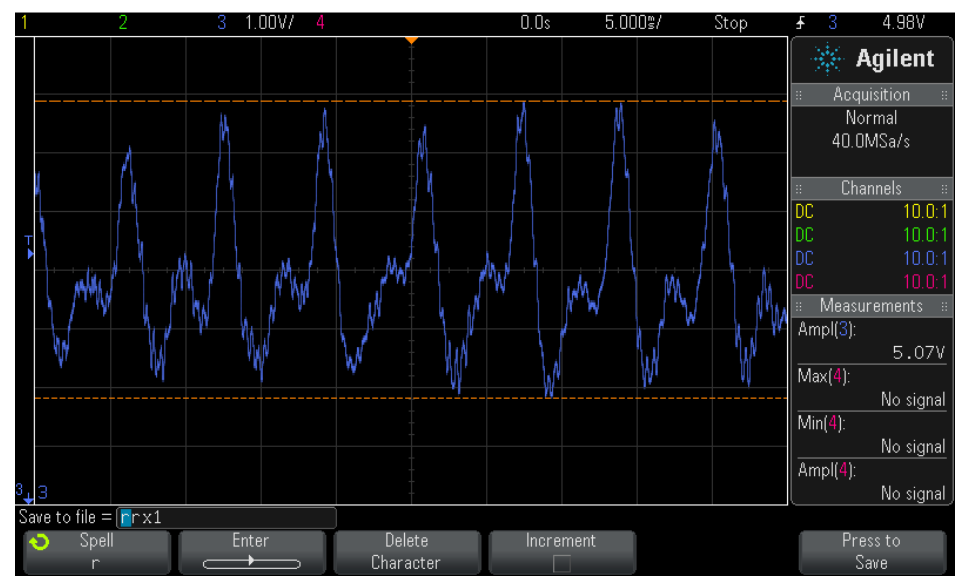

Fig. 3.14: Audio signal amplification and Dc bias addition.

In Fig. 3.14, we can observe the audio signal after amplification and DC bias addition. Then, the modulation IM and the electrical-optical conversion will be done by using LED. A fastswitching time LED would be preferable. The white LED stills an appropriate choice because of its wide use in lighting. In this experiment, the light intensity of a white LED ( $3 W$ High Power Led) acting in range values of $3.2 \mathrm{~V}-9 \mathrm{~V}$ is varied according to the audio signal and transmitted on free space. We can observe from the Fig.3.14, that the DC-bias is about $2 \mathrm{~V}$ and the peak to peak of the amplified audio signal is $5.07 \mathrm{~V}$ where the gain value is 1.85 .

In Fig. 3.15, the received signals at the photovoltaic cell level are presented for separation distance between emitter and receiver of $30 \mathrm{~cm}, 1.05 \mathrm{~m}$ and $2.30 \mathrm{~m}$, respectively. We can observe that, as expected:

- a DC bias is present for all receiving audio signals;

- The received signal intensity is depending on the separation distance. The received signal is fading over a distance. 


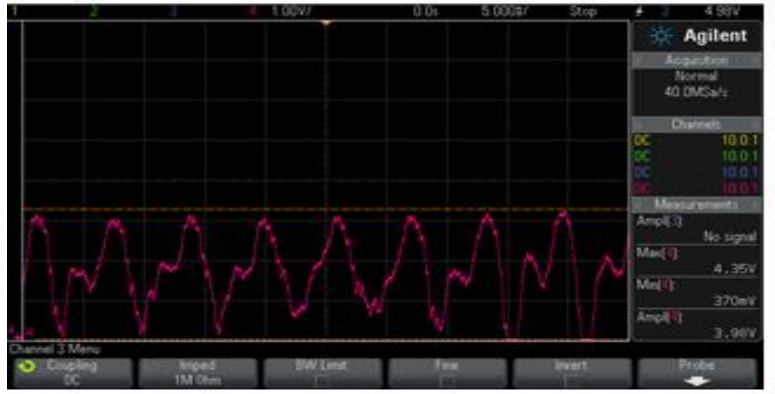

(a)

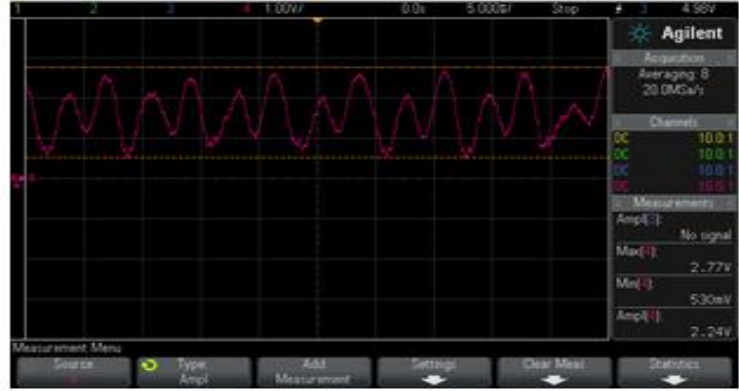

(b)

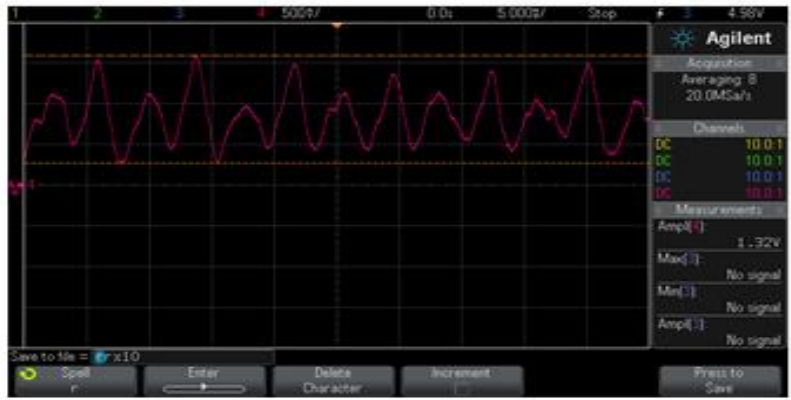

(c)

Fig. 3.15: The received Audio signals with a separation distance between emitter (Led) and receiver (photovoltaic cell) of: (a) $30 \mathrm{~cm}$, (b) $1.05 \mathrm{~m}$ and (c) $2.30 \mathrm{~m}$.

To overcome the fading problem of the received signal, an amplifier should be added just after the photovoltaic cell (Fig. 3.16.a). In this experiment, we have added the LM 385 to amplify the audio signal; it has the ability to amplify 200 times the received signal. The DCBias is removed using a straightened diode bridge and a capacity. To recover and recycle received energy, the DC-Bias is charged in a battery using the mini Solar Lipo charger (CN3065) (Fig. 3.16.b). This latter accepts only input values of $4.4 \mathrm{~V}$ to $6 \mathrm{~V}$ and maximum charge current of $500 \mathrm{~m}$.

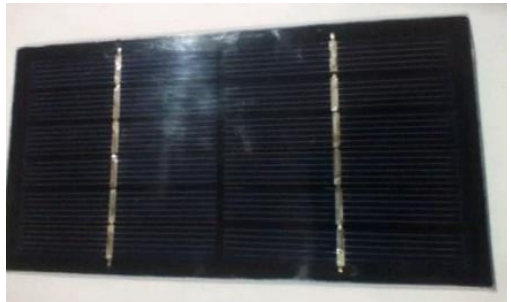

(a)

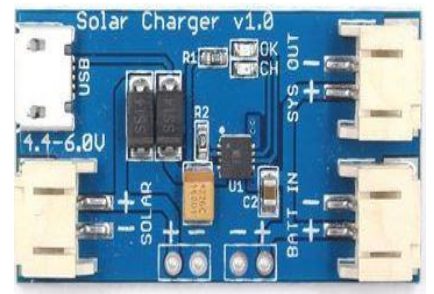

(b)

Fig. 3.16: The photovoltaic cell (JZ-PV11570) and the mini Solar Lipo charger (CN3065).

The ambient light of the room and the sun light received from windows are additional random sources for continuous energy received at the photovoltaic cell.

Another experiment is conducted over a long separating distance between emitter and receiver in indoor environment. We managed to transmit an audio signal using a white LED with respect of the audible quality of the music at the loudspeakers output. We have observed that 
the quality of received signal is good (SNR lower) for a maximum transmission range of about $15 \mathrm{~m}$. However, the audio signal remains audible beyond $15 \mathrm{~m}$. At $25 \mathrm{~m}$ the signal is of course audible, but there is also background noise.

We have measured the light power (in $L u x$ ) received at the photovoltaic cell and the sound power (in $d B S P L$ ) received at the loudspeakers as a function of the wireless distance between transmitter and receiver. The measurements were carried out in a corridor, where the intensity of natural ambient light was 3 Lux.

The curves of the illuminance of the photovoltaic cell and the power of the audio signal received at the loudspeakers as a function of distance are illustrated in Fig. 3.17 and Fig. 3.18 respectively.

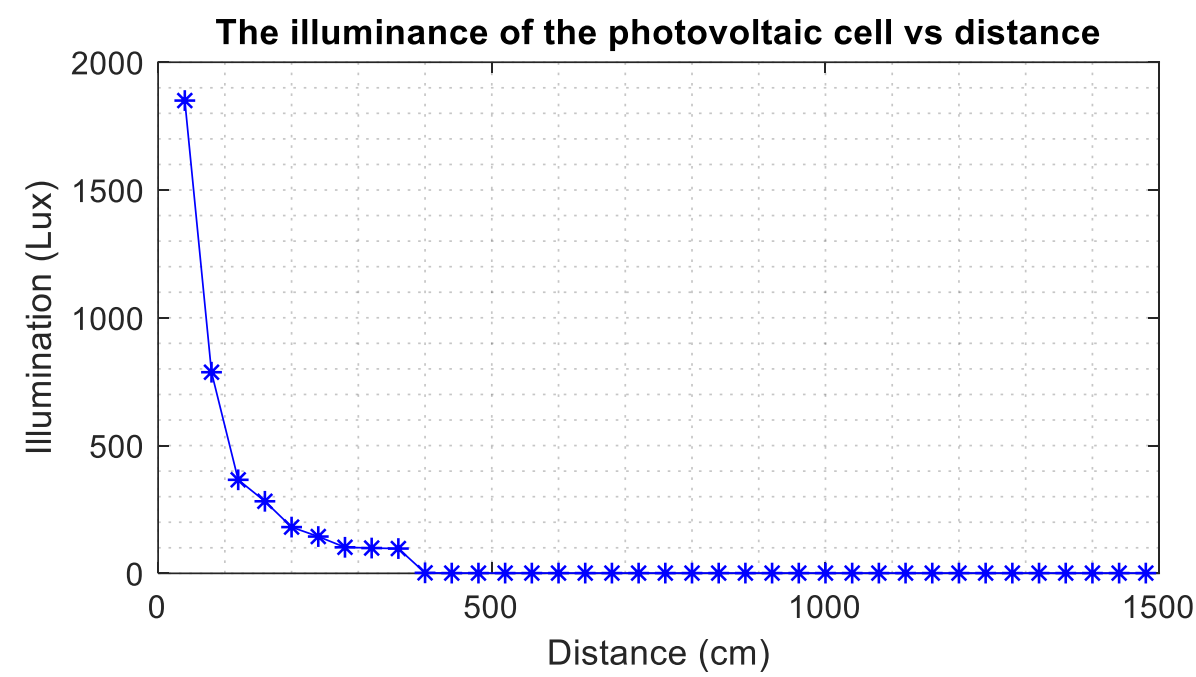

Fig. 3.17: The illuminance of the photovoltaic cell vs the distance between transmitter and receiver.

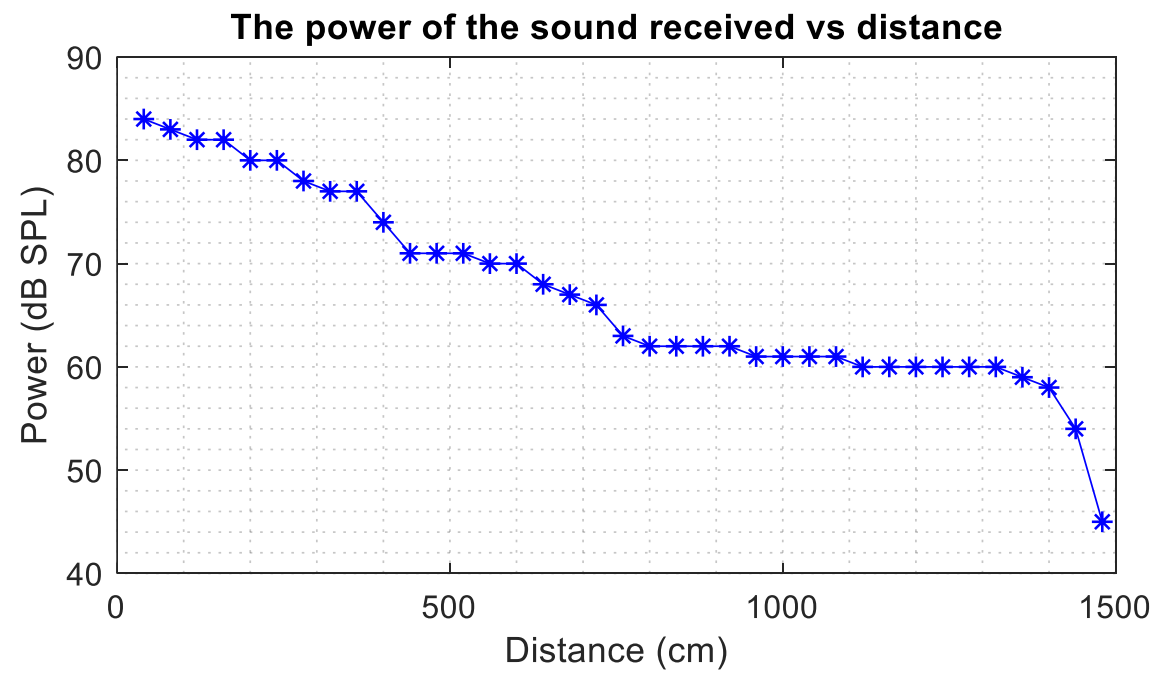

Fig. 3.18: The power of the audio signal received at the loudspeakers vs the distance between the transmitter and the receiver.

From Fig. 3.17 and Fig. 3.18, we have observed that the quality of the received audio signal in loudspeakers and the sensitivity of the photovoltaic cell is good (depending on hearing and 
the quality of the received sound). In fact, even if the separation distance between emitter and receiver is large with a low measured power of the light signal (1 Lux) and the audible powers are from $60 d B S P L$ to $45 d B S P L$, the sound signal can be restored.

This experiment demonstrates the importance of the photovoltaic cell for the future development of long-distance free space communications. This is due to its dual function of energy recovery and receiver of the optical signal.

\subsubsection{Digital wireless transmission using visible light}

In this part, we will present a prototype of digital wireless communication based on visible light. In the area of OWC, research works are focused on achieving faster transmission speeds or producing multiple carrier schemes, while only a few deal with synchronization techniques and transmission protocols. The diagram of an optical wireless communication system is similar to that of a wireless communication system which is composed of: transmitting subsystem, free space channel and receiving subsystem. Successful communication is based on a correct design of the communication system based on the mode of communication, communication protocols and high quality of technical equipment. The synchronization between transmitting subsystem and receiving subsystem is also an important issue in such system.

In the following, the point-to-point optical wireless link will be considered. Then, a general overview of digital communication protocols will be presented, followed by the experimental results.

\subsubsection{Description of Transmitted Data}

In this part, we will focus on transmission of both texts and images using visible light communication links.

- For text transmission, each character has its equivalent in numeric code. Here, the ASCII code (American Standard Code for Information Interchange) is used for the transmission of digital data related to the original text;

- The digital image is represented by a set of dots in matrix form called pixels. For a color image, a color coding system, coupled with a system for representing brightness levels, shall be used to represent the colorimetric information. A digital image is therefore composed of a multitude of pixels and is defined by three terms: the definition, the resolution and the format. Also, different coding systems with their own particularities exist, such as:

○ RGB: based on an additive mixture (combination of light rays) of three primary colors (Red, Green, Blue);

- CMYK: based on a subtractive mixture (combination of color pigments) of three primary colors (Cyan, Magenta, Yellow) and black;

○ HLS: based on the physiological perception of color by the human eye (Hue, Luminance and Saturation). 


\subsubsection{2. $\quad$ Experimental results}

The synoptic of the visible light digital transmission system for indoor environment and simplex mode operation is presented in Fig. 3.19. The operating principle of the VLC system is based on sending digital data via LED in binary code: when the LED is on, bit 1 is transmitted, if the LED is off, bit 0 is transmitted depending on the logic of transmission.

In order to establish a VLC communication, the directivity of the light signal between the transmitter and the receiver must be respected. The transmitter and receiver must be in direct Line-Of-Sight (LOS). Transmission may appear continuous if the frequency of light changes beyond a certain frequency. In fact, the human eye is unable to detect rapid changes in light output. A light-sensitive receiver converts variations in light amplitude into an electrical signal which is then transformed into a high-speed data stream.

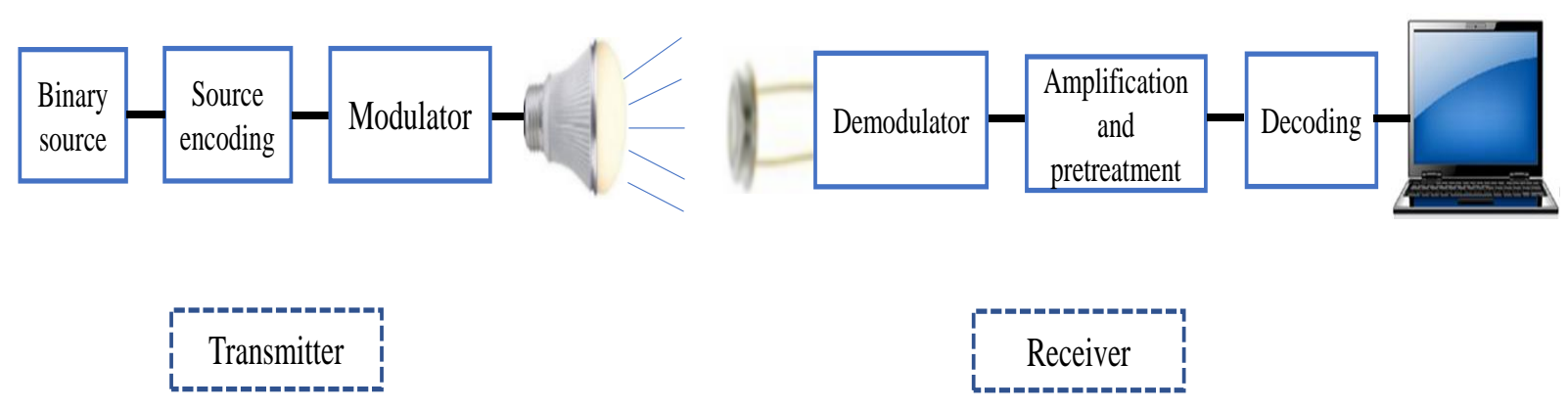

Fig. 3.19: Synoptic diagram of an indoor VLC link in simplex mode.

In transmission part, the binary source is encoded by Manchester type coding. This choice is based on the need to keep the system always in continuous transition between the high level ' 1 ' and the low level ' 0 ' to be able to easily identify the ' 1 ' and ' 0 ' even if the size of the data is large. Then, the OOK modulation is applied to data sent through the LED along the free space link.

In reception, a preamplification is applied to the received signal to prevent from fading of signal over distance. Then demodulation is occurred, and a Manchester-type decoder is applied to get the data which are displayed on the computer.

\section{Material description}

The programming core of our system is the Arduino development board. We have used two cards (Arduino Mega 2560), one for the transmission part, and the second for the reception. The Arduino Mega 2560 is used for the acquisition of the data coming from the PC and the control of the data transmission via visible light.

- Emission part: data are transmitted to the ARDUINO Mega 2560 board for Manchester encoding, OOK modulation and transferring to the optical source. The light signal can be focused with small divergence if a lens is added and an external driver for lenses is required. In addition, the diode switching time is directly determined by Arduino. 
- Reception part: in reception, a photodiode is used as photodetector, this choice is justified by its switching time, which remains the fastest compared to other photodetectors. In the following experiments, a photoreceptor is used because of its high sensitivity to changes in signal state, then; the ARDUINO Mega 2560 card in reception decodes the received data and transmits them to the computer for processing and display.

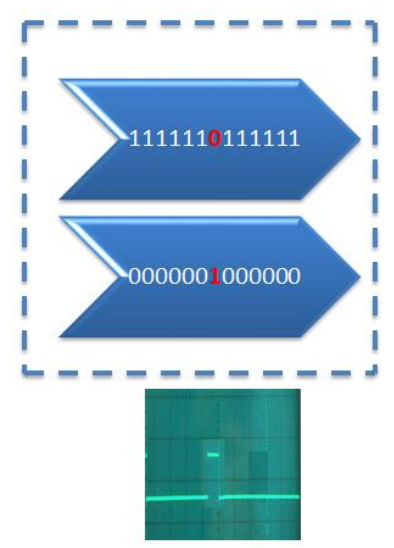

Emission

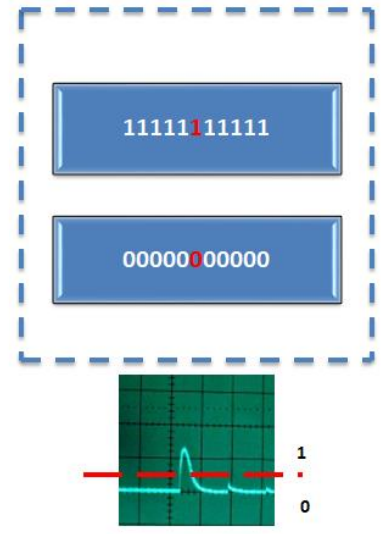

Main Reception Error

Fig. 3.20: Bits detection problem when receiving a succession of identical bits.

\section{Tests and observations}

During the first tests of digital data transmission, we have observed that:

- If the detection threshold of bit " 1 " is fixed, the communication system cannot function properly. We have observed that the detection threshold of bit "1" changes with the distance traveled by the light signal as well as with the environment. This is a consequence of the signal fading over the distance. In addition, the intensity of the transmitted light during the day or night in the same environment is different. Therefore, the detection level of bit "1" must be adaptive to the distance and the environment;

- If a long series of ' 0 ' or ' 1 ' are sent, followed by one change in the state of the bits, the photodiode cannot adapt and the bit error rate (BER) on reception increases (Fig. 3.21). The problem became more complex if the system has to send a large amount of data at a high rate.

\section{Communication protocol}

A communication protocol is established for adaptive bit detection depending on the distance between the transmitter and the receiver. 


\section{a) Proposed algorithm for an adaptive bit detection}

An algorithm for adaptive detection of threshold based on which it decided if the received bit is ' 1 ' or ' 0 ' depending on the received light level has been proposed. The updating of this threshold is automatic, regardless of the distance travelled by the light signal. The performance of this procedure is linked to the sensitivity of the photodiode, the luminous flux and the environment. We have integrated our algorithm in the initial phase before starting data transmission. This algorithm consists of:

1- Sending a sequence of pilot bits of a fixed size, for us, we fix them to 20 bits.

2- Calculation of the separation threshold between the level of bit ' 1 ' and that of bit ' 0 ': 20 bits of random bits ' 1 ' and ' 0 ' are sent. On reception side, the maximum value and the minimum value of the received levels are sought. The threshold is then computed according to the following formula:

$$
\begin{aligned}
& \text { Threshold }=\frac{\text { Maximum Value }+ \text { Minimum Value }}{2} \\
& \text { bit }=\left\{\begin{array}{l}
1 \text { if the received value } \geq \text { Threshold } \\
0 \text { if the received value }<\text { Threshold }
\end{array}\right.
\end{aligned}
$$

\section{b) Manchester encoding}

The source encoding in our experience is Manchester encoding. In this encoding, bit 0 is presented by a rising edge and bit 1 is a falling edge. In this case, permanent transitions are created, this will avoid detection errors. However, the frequency of the encoded signal is double of that of the source signal (Fig. 3.21).

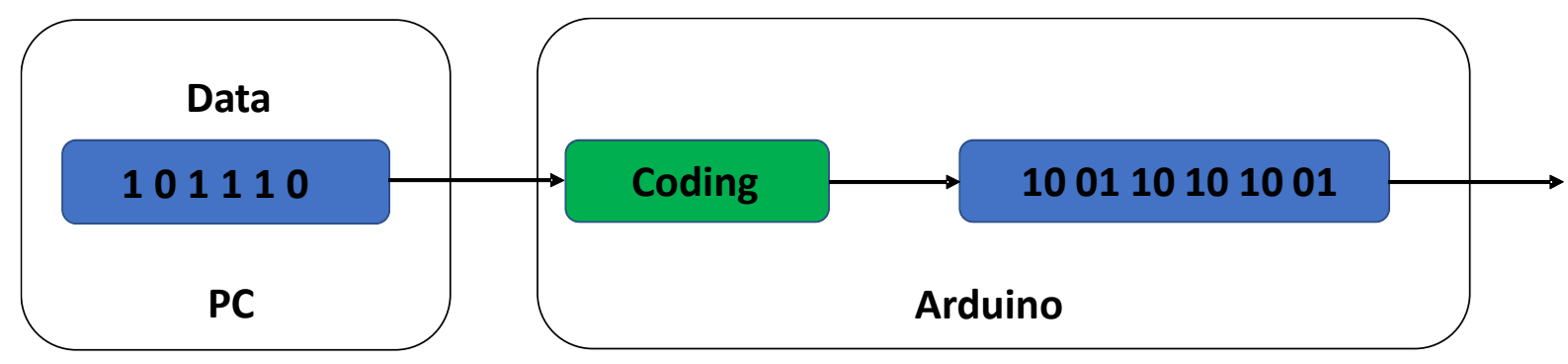

Fig. 3.21: Application of Manchester coding.

\section{c) Synchronization bits}

In 2016-2017, photodiodes with very fast switching time were not available in Algeria, this is why we have decided to set up serial data transmission in simplex mode. Transmission systems requires synchronization between the transmitter and receivers, otherwise there will be a bit shift that will destroy all the information. We therefore provided synchronization bits for each frame of 30 bits to solve the offset problem. Thereafter, our proposed frame of transmission is organized as presented in Fig. 3.22 and the protocol of transmission is presented in Fig. 3.23. 


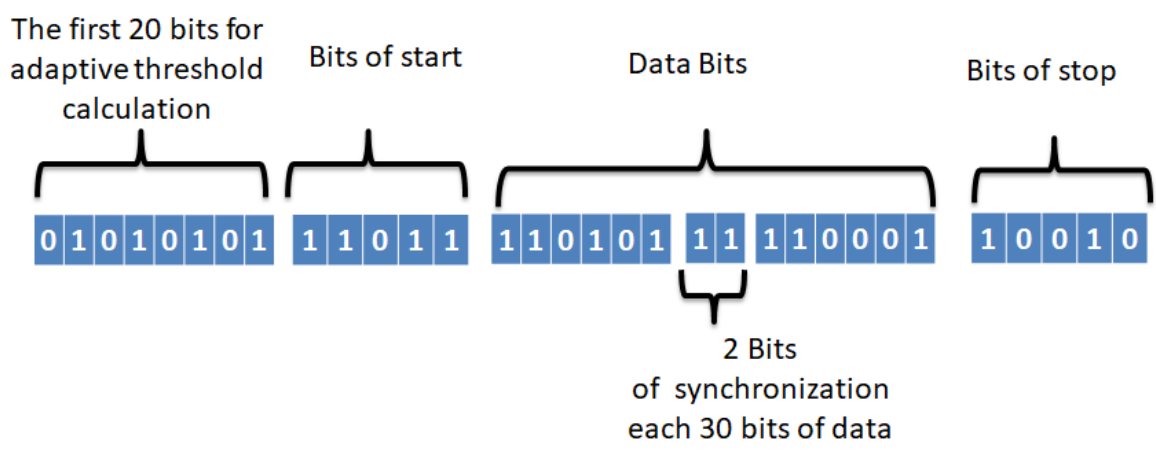

Fig. 3.22: Frame organization in the proposed protocol of communication.

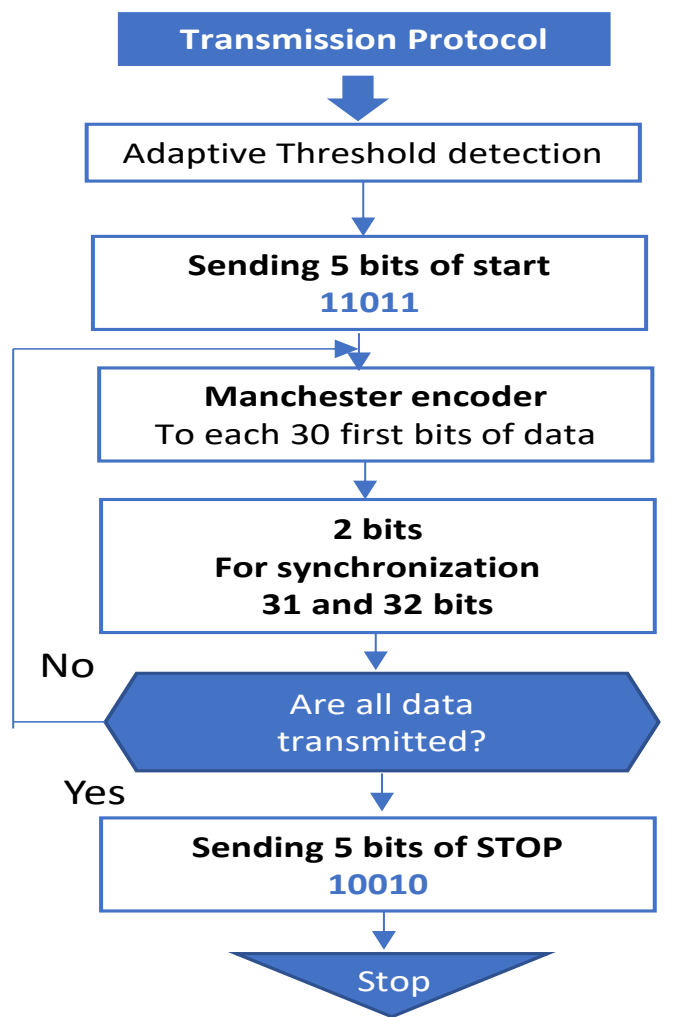

Fig. 3.23: Proposed Protocol of transmission flowchart.

\section{d) Text transmission}

For sending the text, ascii coding is often used whether it is on a PC, mobile phone or other device. We did a direct programming on the Arduino, the serial monitor of the Arduino (IDE) is used as communication interface. It is used to input and display text during sending and receiving. The figure below represents our experimental transmission system. 


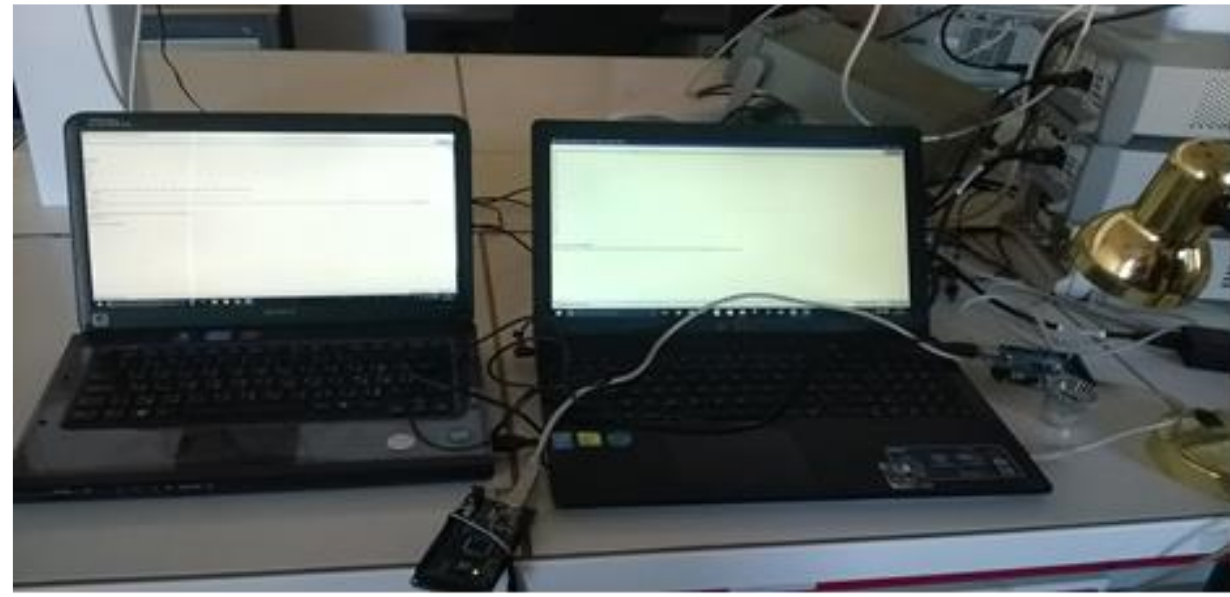

Fig. 3.24: Implementation of our text transmission system via visible light.

Fig. 3.25 shows few examples of texts transmission. On the transmitting PC, we wrote the text we wanted to send to the IDE interface, such as "université mostaganem". On reception, the system transforms the bits into ASCII code and displays them. We found the message was sent via the LED without error as shown in Fig. 3.25.

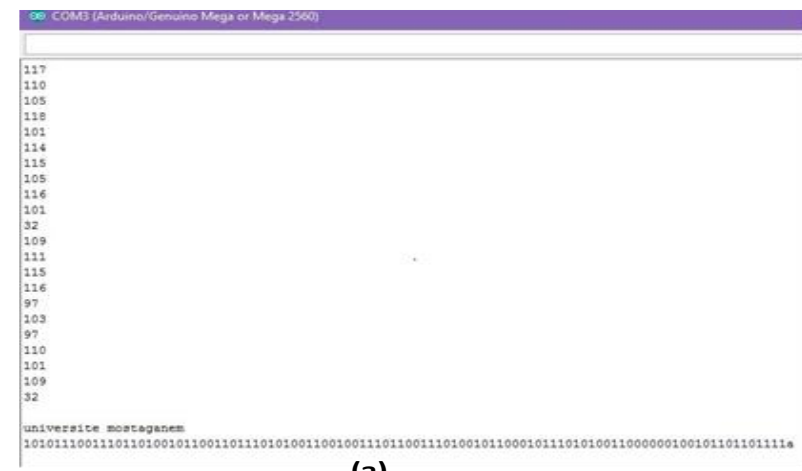

(a)

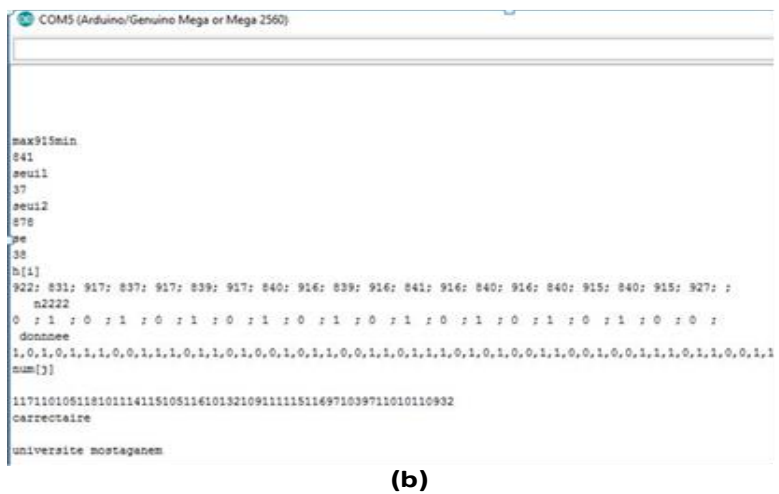

(b)

Fig. 3.25: Example of transmission and reception of a text via visible light. (a) IDE interface of the PC for transmission, (b) IDE interface of the PC reception.

For a better evaluation of the performance of the system, we sent all the letters of the alphabet, messages or words, the maximum bits transmitted in the measurement were 100 bits, the detected errors were null. The following table shows a few examples. 
Table 3.4: Performance of the text transmission system via visible light in detected erros.

\begin{tabular}{|c|c|c|c|}
\hline Word & Emission code & The received code & $\begin{array}{l}\text { detected } \\
\text { Errors }\end{array}$ \\
\hline Bonjour & $\begin{array}{r}010001101111011001110110 \\
010101101111011010101110 \\
01001110\end{array}$ & $\begin{array}{l}010001101111011001110110 \\
010101101111011010101110 \\
01001110\end{array}$ & 0 \\
\hline $\begin{array}{l}\text { université de } \\
\text { mostaganem }\end{array}$ & $\begin{array}{r}101011100111011010010110 \\
011011101010011001001110 \\
110011101001011000101110 \\
100101110000010000100110 \\
1010\end{array}$ & $\begin{array}{r}101011100111011010010110 \\
011011101010011001001110 \\
110011101001011000101110 \\
100101110000010000100110 \\
1010\end{array}$ & 0 \\
\hline laboratoire LEOG & $\begin{array}{r}001101101000011001000110 \\
111101100100111010000110 \\
001011101111011010010110 \\
010011101010011000000100 \\
0011\end{array}$ & $\begin{array}{r}001101101000011001000110 \\
111101100100111010000110 \\
001011101111011010010110 \\
010011101010011000000100 \\
0011\end{array}$ & 0 \\
\hline $\begin{array}{l}\text { communication par } \\
\text { lumiere visible }\end{array}$ & $\begin{array}{r}110001101111011010110110 \\
101101101010111001110110 \\
100101101000011000101110 \\
100101101111011001110110 \\
0000\end{array}$ & $\begin{array}{r}110001101111011010110110 \\
101101101010111001110110 \\
100101101000011000101110 \\
100101101111011001110110 \\
0000\end{array}$ & 0 \\
\hline
\end{tabular}

However, the communication via visible light in free space using the proposed protocol has not got errors if the direct path between the LED and the photodiode is held. The distance between them is about $35 \mathrm{~cm}$. The diagrams of the transmitting and receiving texts are presented in Appendix II.

\section{e) Image transmission}

Since Arduino is not designed to process image data, we were forced to use software that can handle image data processing which language is close to the Arduino language. We therefore used the Visual C++ combined with the special OpenCV library for image processing.

\section{$\checkmark$ The visual $\mathrm{C}++$}

The Visual C++ language and development tools help us to develop native Windows Store applications, native desktop applications and managed applications that run on the .NET Framework and give us the ability to add other libraries, as it is compatible with the majority of libraries that exist either for image processing, sound or other specialized libraries.

\section{$\checkmark$ The OpenCV library}

The OpenCV Library (OpenSource Computer Vision) was originally written in C by researchers from the Intel Company. This library offers a set of more than 2500 algorithms for computer vision, accessible through APIs for the languages $\mathrm{C}, \mathrm{C}++$, and Python. It is now developed, maintained, documented and used by a community of more than 40.000 active 
members. It is the reference library for computer vision in both research and industry. The main modules accessible through its APIC are:

Core: this library is dedicated to basic functionalities. It enables to manipulate basic structures, to perform operations on matrices, to draw on images, to save and load data in XML files...;

> Imgproc: dedicated to image processing. The functions and structures of this module are related to image transformations, filtering, edge detection, points of interest...;

$>$ features2d: dedicated to descriptors. This module mainly concerns the extraction of descriptors according to two common approaches (SURF and StarDetector), interesting for image characterization;

$>$ objdetect: dedicated to object detection. This library allows the recognition of objects in an image using the Adaboost algorithm [Vio 2001];

$>$ video: dedicated to video stream processing. These functions are used to segment and track moving objects in a video;

> highgui: I/O and user interface. OpenCV integrates its own high-level library for opening, saving and displaying images and video streams. It also contains a number of functions to create very simple graphical user interfaces, which are adequated to test our programs;

$>$ calib3d: dedicated to calibration, pose estimation and stereovision. This module contains functions to reconstruct a $3 D$ scene from images acquired with several cameras simultaneously.

However, to transmit a color image, the color information (Red, Green and Blue) has to be recovered for each pixel, converted to binary and grouped into a data vector to be sent in parts to the Arduino serial channel. This is due to the limited memory of the Arduino, which cannot save the full size of the image vector, so the data is sent in frames. The program flow diagrams for the transmission and reception of the images are shown in Appendix III.

At the Arduino level, we have used the same communication protocol as the one used to send the text: calculation of the bit detection threshold, synchronization, coding and decoding. During the transmission and reception of the image, the Arduino receives the transmitted bits without processing or reorganizing the frames. These tasks have already been performed by $\mathrm{C}++$ as well as the processing and display of the image on reception.

At the end of all these operations, we manage to transmit an image from one computer to another via visible light without any error. This shows the reliability and performance of our system even with large data sizes. Fig. 3.26 illustrates the transmission of an image via visible light. 


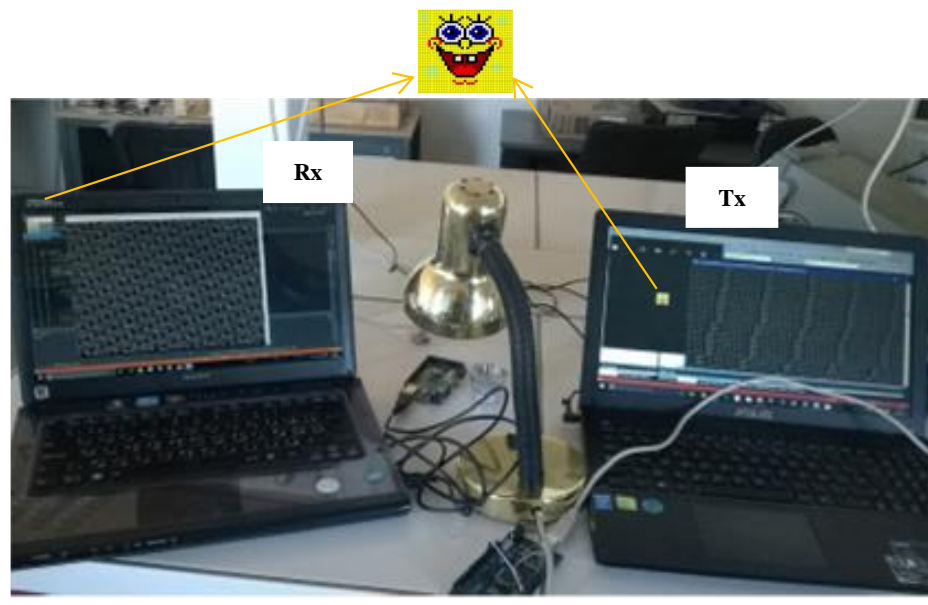

Fig. 3.26: Transmission of an RGB image of size $(50 \times 50)$ using visible light in free space.

The visualization on the digital oscilloscope of the signals emitted and those received are shown in Fig. 3.27. We notice that the emitted signal is a digital pulse train, while for the received signal; each pulse becomes a charge and discharge due to the behavior of the photodiode.

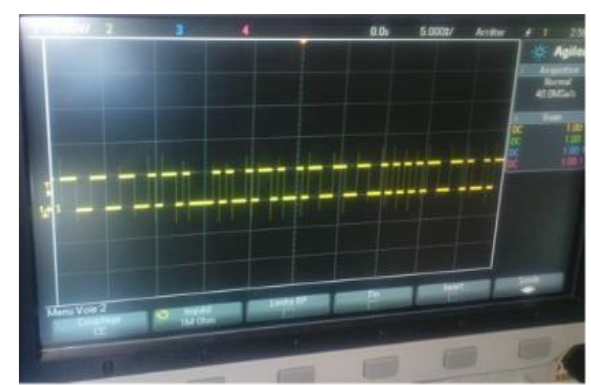

(a)

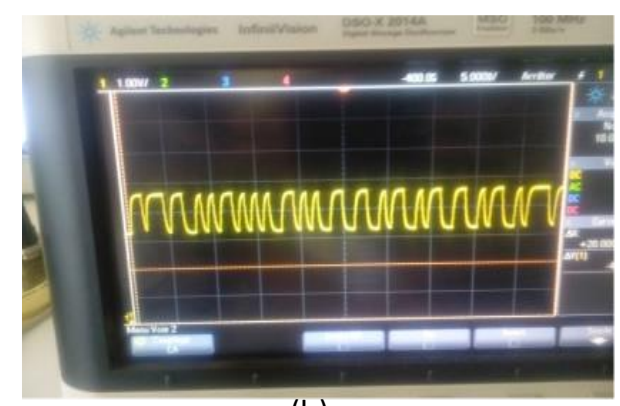

(b)

Fig. 3.27: Oscilloscope visualization of the emitted signal after Manchester encoding and the received signal from the photodiode.

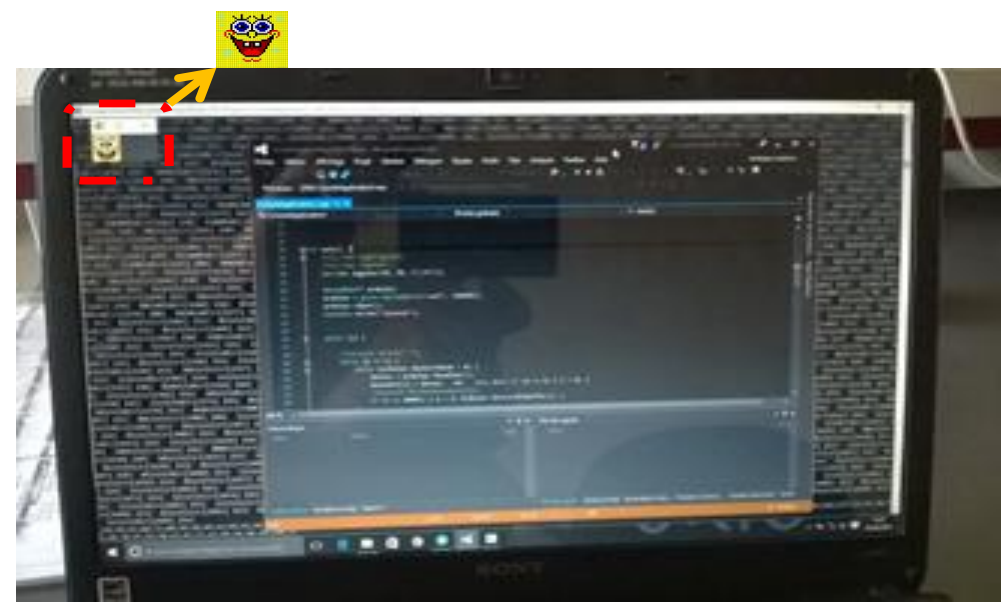

Fig. 3.28: Image reconstitution on the computer screen. 


\subsection{Conclusion}

In this chapter, we have presented a brief summary of OWC systems as well as the simplest modulation techniques used in the free optical communication systems. We have demonstrated that an OWC system can be used not only for data transmission, but also for wireless energy transmission. A communication protocol ensuring reliable numerical data transmission with an adaptive bit level detection algorithm is proposed. Its effectiveness has been demonstrated by the transmission of texts and images. 


\section{Chapter 4}

\section{Optical OFDM signal transmission}

using a broadband source over SSMF 


\subsection{Introduction}

Over these past years, fiber-optic communications technology has been developed rapidly. However, there are some penalties in the Single-Mode Fibers (SMFs) and Multi-Mode Fibers (MMFs) links which lead to lower bitrates than those required for current and emerging demand. Optical Orthogonal Frequency Division Multiplexing (OOFDM) has been investigated within the last few years by extensive simulations and experiments [Hao 2009]. OOFDM has been considered as a promising candidate for the optical communications systems, such as visible light communication (VLC) systems [Gha 2012] and optical fiber communication [Gid 2010], since it combines the advantages of wireless OFDM systems and optical communications which including dynamic provision of hybrid bandwidth allocation in both the frequency and time domains, significant reduction in network complexity and potential for cost-effective implementation. This modulation technique has been found to exhibit very high tolerances to chromatic dispersion and polarization mode dispersion (PMD) [Shi 2007].

Currently, there are two main implementations for OOFDM: coherent optical OFDM and direct-detection optical OFDM (DD-OOFDM). The latter is the simplest approach for optical OFDM systems and is especially suitable for metropolitan and access networks [Wei 2009], where CO-OFDM offers better sensitivity and spectral efficiency but higher complexity and costs [Shi 2006]. Recently, several experimental results on OOFDM system have been reported, such as a long-distance transmission of $25.8 \mathrm{~Gb} / \mathrm{s}$ over $4160 \mathrm{~km}$ of SSMF has been demonstrated [Jan 2007], an experimental demonstration of $7.09 \mathrm{~Gb} / \mathrm{s}$ directly modulated laser (DML) based Real-time Optical OFDM Transmission over 50km SSMF [Zha 2016] and a demonstration of the single-channel direct detection beyond $1 \mathrm{~Tb} / \mathrm{s}$ with $1.26 \mathrm{~Tb} / \mathrm{s}$ line rate and $1.02 \mathrm{~Tb} / \mathrm{s}$ net rate after $100 \mathrm{~km}$ SSMF [Che 2019].

In the case of a multipath channel, conventional modulation techniques are very sensitive to Inter Symbol Interference (ISI). OFDM is a digital multi-carrier transmission technique that has shown strong performances since it was first presented by Chang of Bell labs in a 1966 US patent [Arm 2009]. The OFDM modulation is a special case of multi-carrier modulation (MCM) [Wei 1971]. It is a modulation technique which consists of distributing the digital signal to be transmitted over many subcarriers. The generation of intensity modulated and directly detected (IM/DD) OFDM signals employs either double-sideband (DSB) modulation, which occupies a larger bandwidth in the optical spectrum and suffers from chromatic dispersion induced power fading, or single sideband (SSB), which is preferably employed due to its lack of power fading and more efficient usage of the spectrum at the expense of the SSB modulation complexity [Lin 2012]. The OFDM modulation is currently used as the multicarrier digital modulation technique of choice in Passive Optical Networks (PONs) because of its robustness to chromatic dispersion and other impairments, as well as its high spectral efficiency and flexible bandwidth allocation [Arm 2009].

Moreover, optical broadband sources (BBS) are attractive due to their larger cost effectiveness [Kan 2014] than wavelength specific sources, i.e. lasers, and also the major robustness to crosstalk and temperature drift compared to other components. However, it is well known that fiber chromatic dispersion limits the performance of extended reach WDM- 
PONs. A subcarrier multiplexing tolerant dispersion transceiver based on optical broadband sources was presented in [Gra 2009] and offers the prospect of Radio over fiber (RoF) signal transmission by using spectrum-sliced incoherent light sources such as amplified spontaneous emission (ASE) or light-emitting diodes (LEDs) in WDM networks. Accordingly, a low-cost OOFDM signal transmitter based on an optical broadband source was demonstrated for moderate bitrate signal transmission in WDM access networks [Chi 2017b].

In this chapter, an overview of broadband sources based optical networks will be given, such as a description of the different broadband sources, fundamentals of signal transmission based on BBSs and tolerant dispersion systems for BBS based optical links. The chapter includes the description of generation and reception of OFDM signals followed by a discussion on the optical components. Then, we focused on the design of an OOFDM-WDM experimental network using a broadband source over SSMF and the experimental setup will be explained. Finally, based on the achieved results, the main observations will be summarized in the conclusion.

\subsection{Broadband sources based optical networks}

\subsubsection{Different broadband sources}

A classification of optical sources according to the method used for light generation [Cap 1998, Chi 2018] leads to lasers and optical broadband sources (BBS). Lasers are optical sources emitting coherent light at a given wavelength with narrowband spectral bandwidth, which exhibit a low divergence angle that spreads out very little as it projects out further from its source. They are based on an oscillator where the amplification is due to the stimulated emission and the feedback is determined by the structure of the cavity. On the contrary, broadband sources either use the spontaneous emission for lightwave generation or use the amplified spontaneous emission of diode-pumped, erbium-doped fibers, and they emit a very stable, wide and flat spectrum in the output range up to $+23 \mathrm{dBm}$.

Therefore, among the BBS, we can distinguish the Light-emitting diodes (LEDs), which are available at a reasonable cost with widths between 50 and $100 \mathrm{~nm}$, superluminescent LEDs (sLEDs) with smaller lines and higher optical output powers and Amplified Spontaneous Emission (ASE) noise sources, with larger lines.

The following table shows the linewidths from different sources [Cap 1998, Ram 2002, Chi 2018]. 
Table 4.1: Typical linewidth of different optical sources [Chi 2018]. DFB: Distributed FeedBack, DBR: Distributed Bragg Reflector, FP: Fabry-Perot, ECL: External Cavity Laser, VCSL: Vertical-Cavity SurfaceEmitting Laser, MWQ: Multiple Quantum Well, sLED: superluminescent Light-Emitting Diode, LED: LightEmitting Diode, ASE: Amplified Spontaneous Emission.

\begin{tabular}{|c|c|c|}
\hline Classification & Source & Linewidth \\
\hline \multirow{4}{*}{ Narrow } & DFB & $1-10-\mathrm{MHz}$ \\
\cline { 2 - 3 } & DBR & $1-10 \mathrm{MHz}$ \\
\cline { 2 - 3 } & FP & $2-5 \mathrm{~nm}$ \\
\cline { 2 - 3 } & ECL & $10-100 \mathrm{kHz}$ \\
\cline { 2 - 3 } & VCSEL & $40-150 \mathrm{MHz}$ \\
\cline { 2 - 3 } & MWQ & $100-300 \mathrm{kHz}$ \\
\hline \multirow{3}{*}{ Wide } & SLED & $35-40 \mathrm{~nm}$ \\
\cline { 2 - 3 } & LED & $50-100 \mathrm{~nm}$ \\
\cline { 2 - 3 } & ASE & $80 \mathrm{~nm}$ \\
\hline
\end{tabular}

As will be explained in the following section, the impact of fiber dispersion when broadband sources are employed is very significant and therefore operating bandwidths, i.e. maximum bitrates are seriously limited. Recently, the utilization of a tunable Mach-Zehnder interferometer (TMZI) allowed to demonstrate an OOFDM access network based on a single optical broadband source (BBS) to transmit moderate bitrates with low cost, high stability, low crosstalk operation [Chi 2017a, Chi 2017b] and also allowing multiband OFDM transmission in WDM networks [Chi 2017a, Chi 2017b]. A theoretical analysis in [Gra 2009] details the potential of this optical BBS based transmission system over a radio frequency band, also free from carrier suppression effect for any amplitude modulation format. In this context, we explore the large potentiality offered by the BBS to implement a bidirectional reconfigurable network with dynamic bandwidth allocation, multiple bands operation and multiple users [Sek 2018].

\subsubsection{Fundamentals of signal transmission based on BBSs}

The employment of BBS is of enormous technical interest because of their great potential for enabling multi-channel applications in WDM-PONs [Jun 1998, Kim 2007, Chi 2018] while minimizing the implementation and management costs associated to the generation of the WDM channel.

However, signal propagation on SSMF links is affected by the presence of the chromatic dispersion parameter of the fiber. The dispersion effect is not only dependent on the fiber characteristics but is also related to the optical modulation process and the spectral characteristics of the optical source. Therefore, the use of a BBS will impose significant limitations. The following figure shows a schematic of an optical transmission system using the external modulation of a BBS. 


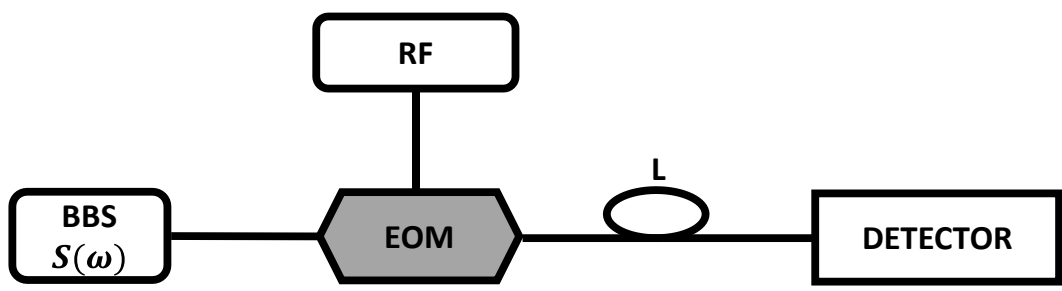

Fig. 4.1: Schematic of a transmission system using an optical broadband source.

Let $S(\omega)$ be the power spectral density of the optical signal emitted by a BBS characterized by its spectral width $\delta \omega_{3 d B}$. When the lightwave signal is amplitude modulated by an RF signal of frequency $f$ using a double sideband and transmitted along an optical fiber link of length $L$ with dispersion parameter $\beta_{2}$, as illustrated in Fig. 4.1, the electrical transfer function $H^{R F}(\Omega)$ is determined by [Gra 2009]:

$$
H^{R F}(\Omega) \propto C S E(\Omega) . H_{0}^{R F}(\Omega)
$$

Where $\boldsymbol{\Omega}=\mathbf{2} \boldsymbol{\pi} \boldsymbol{f}, \boldsymbol{C S} \boldsymbol{E}(\boldsymbol{\Omega})$ represents the electrical carrier suppression effect defined as:

$$
\operatorname{CSE}(\Omega)=\cos \left(\frac{1}{2} \beta_{2} L \Omega^{2}\right)
$$

And $H_{0}^{R F}(\Omega)$ is defined as:

$$
H_{0}^{R F}(\Omega)=\frac{\int_{-\infty}^{+\infty} s(\omega) \exp \left\{-j \beta_{2} L\left(\omega-\omega_{0}\right) \Omega\right\} d \omega}{\int_{-\infty}^{+\infty} S(\omega) d \omega}
$$

As shown in eq. (4.3), the power spectral density of the BBS strongly determines the electrical transfer function. Also note the relationship in terms of Fourier transforms between the transfer function $H_{0}^{R F}(\Omega)$ and the power spectral density $S(\omega)$ [Gra 2009].

In the case of an optical source with a Gaussian profile, which describes the typical spectral power distribution of LEDs, the power spectral density is described as:

$$
S(\omega)=\frac{P_{0}}{\sqrt{2 \pi}} \frac{1}{\delta \omega} \exp \left\{-\left(\frac{\omega-\omega_{0}}{\delta \omega}\right)^{2}\right\}
$$

Where $\boldsymbol{\delta} \boldsymbol{\omega}=\frac{\boldsymbol{\delta} \boldsymbol{\omega}_{3 d B}}{2 \sqrt{\ln 2}}, P_{0}$ and $\delta \omega_{3 d B}$ are the total average power and the $3 d B$ optical linewidth of the source, respectively. The expression of the transfer function in eq. (4.3) results as:

$$
H_{0}^{R F}(\Omega)=\exp \left\{-\left(\frac{\beta_{2} L \Omega \delta \omega}{2}\right)^{2}\right\}
$$

And therefore, we find that this profile exhibits an exponential dependence.

Moreover, the power spectral density of an optical broadband source with a rectangular profile can be expressed as:

$$
S(\omega)=\left\{\begin{aligned}
\frac{P_{0}}{\delta \omega_{3 d B}}, & \left|\omega-\omega_{0}\right|<\frac{\delta \omega_{3 d B}}{2} \\
0, & \left|\omega-\omega_{0}\right|>\frac{\delta \omega_{3 d B}}{2}
\end{aligned}\right.
$$

which leads to the following electrical transfer function: 


$$
H_{0}^{R F}(\Omega)=\operatorname{sinc}\left(\frac{\beta_{2} L \Omega \delta \omega_{3 d B}}{2}\right)
$$

The width of the optical source has a huge impact on the electrical response and on its electrical bandwidth of $3 d B$. Indeed, for a given optical link, the increasing width of the optical source produces a decrease of the $3 d B$ bandwidth in the electrical domain allowing only a few $\mathrm{MHz}$ [Gra 2013] of signal transmission. This effect is given by the chromatic dispersion parameter in combination with the source width and is represented by the term $H_{0}^{R F}(\Omega)$ defined in equation eq. (4.3).

The electrical $3 d B$ bandwidth of the RF bandpass in our system can be easily obtained in terms of the optical carrier bandwidth and the dispersion characteristic of the link. According to the rectangular profile of eq. (4.6), the bandpass window has a $3 d B$ electrical bandwidth $\delta f_{3 d B}$ can be obtained by:

$$
\delta f_{3 d B} \sim \frac{0.9 \lambda^{2}}{\left(\beta_{2} L\right) 2 \pi c \delta \lambda_{3 d B}}
$$

Fig. 4.2 represents the theoretical transfer functions $H_{0}^{R F}(f)$ for a link with $\beta_{2}=$ $-20 \mathrm{ps}^{2} / \mathrm{km}, L=10 \mathrm{~km}$ at $1550 \mathrm{~nm}$ and different source widths $\delta \omega_{3 \mathrm{~dB}}$, for a source with rectangular and Gaussian profiles [Chi 2018].
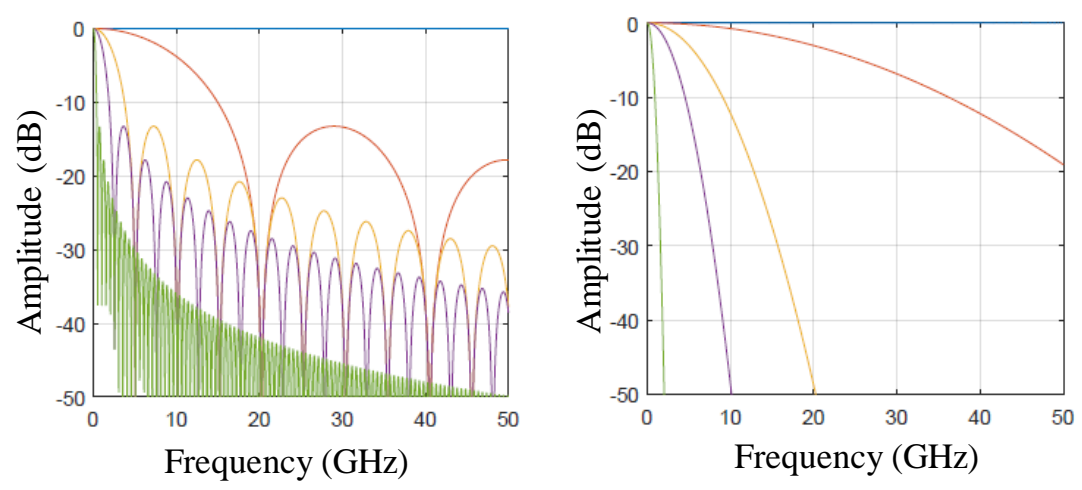

Fig. 4.2: Transfer functions using a broadband source with different values of $\left(\delta \omega_{3 d B}\right) / 2 \pi[\mathrm{GHz}]: 0.125$ (blue), 12.5 (Orange), 50 (yellow), 100 (violet), 500 (green) in cases (a) Rectangular distribution and (b) Gaussian distribution.

As shown in Fig. 4.2 the theoretical frequency behavior is strongly influenced by the bandwidth of the optical source $\delta \omega_{3 d B}$, resulting in an electrical low-pass filtering response. The values of the $-3 d B$ bandwidth frequency response, defined as Full Width at Half Maximum (FWHM), are presented in Table 4.2. 
Table 4.2: Optical source with different width, $\delta \omega_{3 d B} / 2 \pi$, values used in the theoretical model and corresponding FWHM widths of the electrical transfer function for rectangular and Gaussian sources.

\begin{tabular}{|l|l|c|c|c|c|c|}
\hline \multicolumn{2}{|c|}{ Width of source $\left(\delta \omega_{3 d B}\right) / 2 \pi[\mathrm{GHz}]$} & $\rightarrow 0$ & 12.5 & 50 & 100 & 500 \\
\hline \multicolumn{2}{|c|}{$\delta \lambda(\mathrm{nm})$} & 0.001 & 0.1 & 0.4 & 0.8 & 4 \\
\hline \multirow{2}{*}{$\begin{array}{l}\text { FWHM } \\
(\mathrm{GHz})\end{array}$} & Rectangular distribution & $\rightarrow \infty$ & 9 & 2.24 & 1.12 & 0.22 \\
\cline { 2 - 8 } & Gaussian distribution & $\rightarrow \infty$ & 19.85 & 4.96 & 2.48 & 0.50 \\
\hline
\end{tabular}

\subsubsection{Tolerant dispersion systems for BBS based optical links}

Due to the limitations of the broadband sources, they have not been widely used in WDMPON systems. The literature related to the optical transmission of OFDM signals mostly employs narrow sources, for both direct detection and coherent detection. However, the use of broadband sources can be an interesting low-cost solution. The use of a transmission structure based on Mach-Zehnder Interferometer (MZI) with Variable Delay Line (VDL) to overcome the limitations of broadband sources in RF signal transmission has been studied by [Gra 2009]. In [Chi 2018], a low-cost solution for the implementation of WDM-OFDM transmitters based on the use of broadband sources for the transmission of OOFDM signals was proposed and realized.

The MZI is an optical device consisting of two $3 d B 1 \times 2$ optical power couplers, one at the input and the other at the output, with a variable delay line (VDL) inserted in one of the two fiber arms which causes different delay to the signals travelling along both arms. The schematic representation is given in Fig. 4.3.

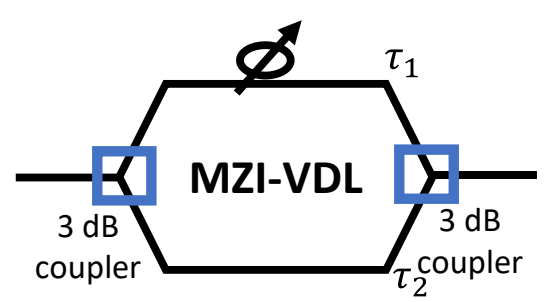

Fig. 4.3: Transmission structure based on a Mach-Zehnder interferometer and a VDL.

The MZI-VDL can be introduced just after the broadband source. However, as indicated in [Gra 2013], the insertion of the MZI-VDL after the modulator allows carrier suppression effects to be attenuated, and therefore, bandwidth for RF signal transmission is increased. Fig. 4.4 illustrates the transmission scheme.

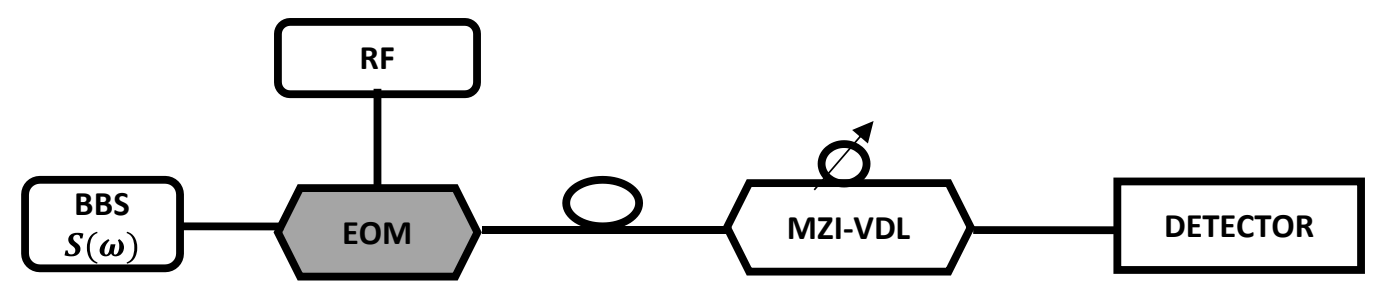

Fig. 4.4: Scheme of transmission with Broadband Sources based on Mach-Zehnder interferometer. 
The transfer function of the MZI-VDL is [Cap 1999]:

$$
H_{M Z I-V D L}(\Omega)=\frac{1}{\sqrt{2}}\left[e^{-j\left(\Omega-\Omega_{0}\right) \tau_{1}}+e^{-j\left(\Omega-\Omega_{0}\right) \tau_{2}}\right]
$$

Where $\tau_{1}$ and $\tau_{2}$ are the optical delay for each MZI branch, and the frequency $\Omega_{0}$ is given by:

$$
\boldsymbol{\Omega}_{\mathbf{0}}=\frac{\Delta \tau}{\boldsymbol{\beta}_{2} L}
$$

Where $\Delta \tau=\left|\tau_{1}-\tau_{2}\right|$.

In this case, the expression of the transfer function (eq. (4.1)) undergoes a slight variation, resulting:

$$
\begin{aligned}
H^{R F}(\Omega) & \propto \cos \left(\Omega \frac{\Delta \tau}{2}\right) \cdot \operatorname{CSE}(\Omega) \cdot H_{0}^{R F}(\Omega)+ \\
& +\frac{C S E^{-}}{2} \cdot H_{0}^{R F}\left(\Omega-\Omega_{0}\right)+\frac{C S E^{+}}{2} \cdot H_{0}^{R F}\left(\Omega+\Omega_{0}\right)
\end{aligned}
$$

Where $C S E^{\mp}$ is defined as follows:

$$
C S E^{\mp}=\cos \left(\frac{\beta_{2} L \Omega\left(\Omega \mp \Omega_{0}\right)}{2}\right)
$$

The eq. (4.12) shows clearly that the CSE function appears now frequency shifted by an amount which depends on the central frequency $\Omega_{0}$ given by eq. (4.9). Indeed, when $\Omega=\Omega_{0}$, the cosine function would give a constant and unitary value, however, the CSE is negligible. Moreover, it is possible to deduce from the terms $H\left(\Omega \mp \Omega_{0}\right)$ the appearance of a new RF transmission window centered on the frequency $\Omega_{0}$ (see eq. (4.10)). According to this fact, the center frequency of this window can be varied by changing three parameters: the delay between the arms of the interferometer, $\Delta \boldsymbol{\tau}$, the dispersion parameter of the fiber, $\beta_{2}$, and the length of the link, $L$. Thus, the carrier suppression effect has been effectively mitigated for a DSB transmission scheme based on broadband sources.

Fig. 4.5(a) and 4.5(b) plot the optical output spectrum of the broadband source and the optical spectrum at the output of the optical filter with $6 \mathrm{~nm}$ bandwidth, respectively.

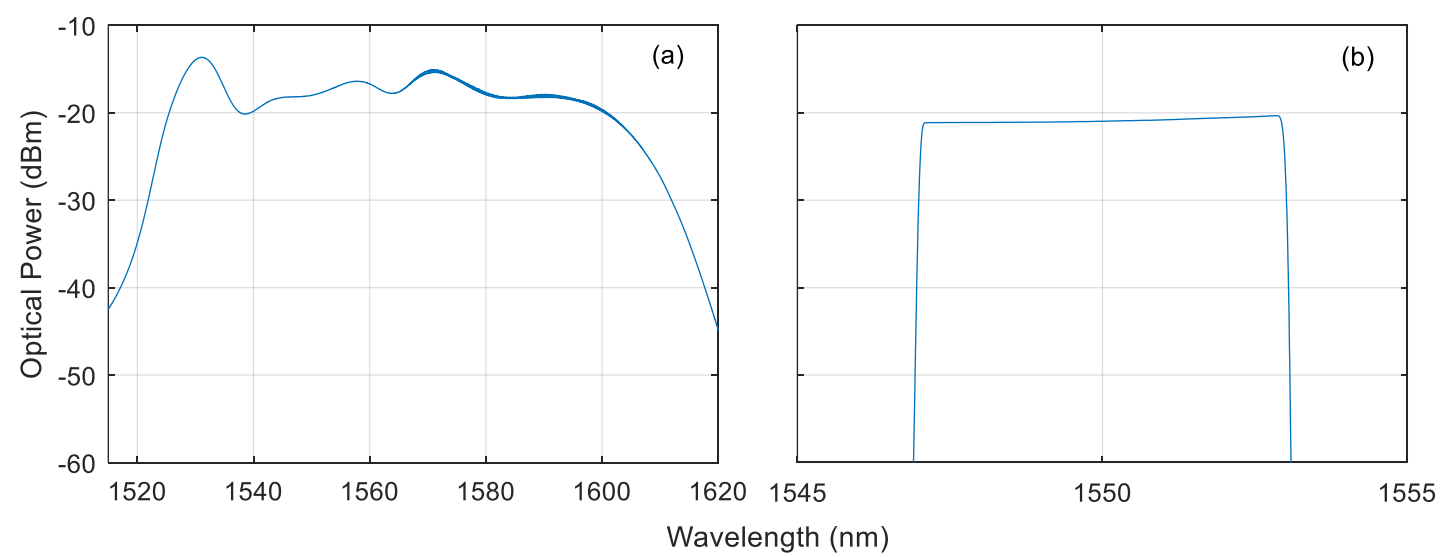

Fig. 4.5: (a) Optical spectrum of the broadband source, (b) optical spectrum at the output of the $6 n m$ bandwidth optical filter. 
In the following, we will see the effect of the introduction of MZI-VDL, illustrated previously in Fig. 4.4, in the transmission system employing the $6 \mathrm{~nm}$ bandwidth optical source depicted in Fig. 4.6.b). The source is amplitude modulated by an RF signal. The modulated signal is transmitted through an optical link of $L=10 \mathrm{~km}$, whose dispersion parameter is determined by $\beta_{2}=-20 \mathrm{ps}^{2} / \mathrm{km}$. The MZI-VDL is configured to allow the transmission of the band centered at $5 \mathrm{GHz}$, so according to eq. (4.10), a delay between the arms of the interferometer is required around $\Delta \tau=6.28 \mathrm{ps}$. Fig. 4.7 shows the optical spectrum at the output of the MZI-VDL and the corresponding electrical transfer function are illustrated in the following figure.
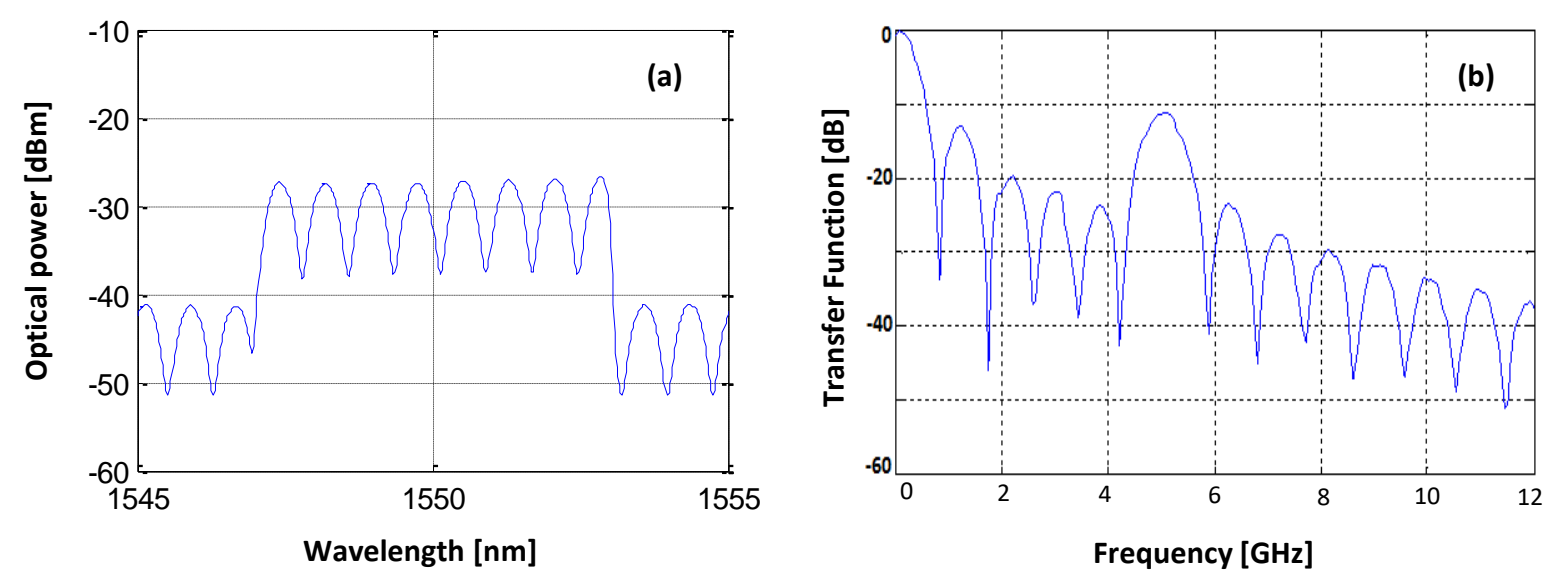

Fig. 4.6: (a) Optical spectrum at the output of the MZI-VDL. (b) Electrical transfer function of the proposed system.

The value of the delay between the arms of the MZI-VDL can be obtained directly from the optical spectrum (Fig. 4.6a) and by using the value of the interval between the rise $(1546.86 \mathrm{~nm})$ and the fall $(1553.14 \mathrm{~nm})$ of the signal. On the other hand, Fig. $4.6 \mathrm{~b}$ shows the RF transmission band opened at the center frequency given by eq. (4.9) due to the introduction of the MZI-VDL. 


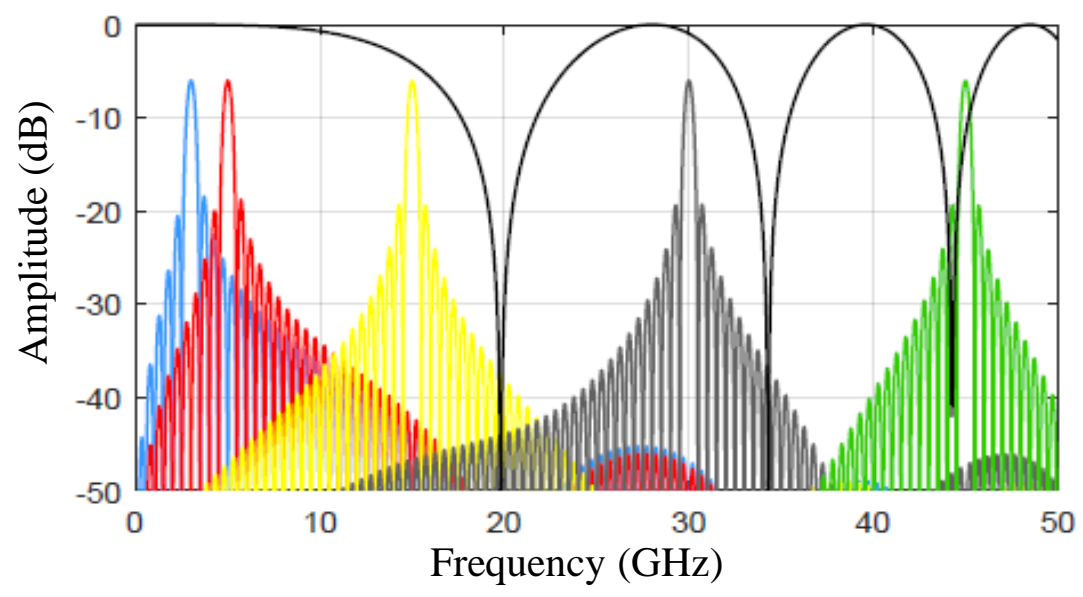

Fig. 4.7: The response of the transfer function for different $\Delta \tau$ (blue: $6.38 p s$, red: $3.83 p s$, yellow: $19.13 p s$, grey: $38.26 \mathrm{ps}$ and green: $57.40 \mathrm{ps}$ ).

At high data rates, dispersion-induced widening of short pulses in the fiber causes crosstalk between adjacent time slots, leading to errors as the communication distance increases beyond the dispersion length of the fiber. However, higher order dispersion terms are the harmful forces in a very high-speed optical transmission system.

Different order dispersion terms result from the nonlinear frequency dependence of the propagation constant given by the Taylor expansion:

$$
\beta(\omega)=\beta_{0}(\omega)+\frac{\delta \beta}{\delta \omega}\left(\omega-\omega_{0}\right)+\frac{1}{2} \frac{\delta^{2} \beta}{\delta \omega^{2}}\left(\omega-\omega_{0}\right)^{2}+\frac{1}{6} \frac{\delta^{3} \beta}{\delta \omega^{3}}\left(\omega-\omega_{0}\right)^{3}+\cdots
$$

where $\omega$ is the angular frequency [Agr 1995]. The last two terms of the equation represent the dispersive terms, where both group velocity dispersion (GVD), $\boldsymbol{\beta}_{\mathbf{2}}$, and the third order dispersion (TOD), $\boldsymbol{\beta}_{3}$, related to the dispersion slope, are expressed in terms of angular frequency or equivalently, in terms of wavelength, as follows:

$$
D=-\frac{2 \pi c}{\lambda^{2}} \beta_{2} ; \quad S=\left(-\frac{2 \pi c}{\lambda^{2}}\right)^{2} \beta_{3}
$$

However, careful management of GVD and TOD is essential to achieve consistent performance across the optical link transmission.

The nonlinear nature of the optical modulation process produces harmonics on both sides of the optical carrier. Hence, the optically modulated wave undergoes different impairments while propagating through the RoF link. The nonlinearity of the electrical-optical modulator, nonlinearities in the optical fiber and weak modulating process result in fragile optical signals [Lac 2002]. To resolve the issue of pulse broadening from dispersion, several compensators have been demonstrated for second-order [Oue 1995] and third-order [Dur 1997, Lee 2007, Che 2014, Gao 2017, Ben 2018] dispersion, mainly using optical fiber platforms. Several dispersion-managed (DM) techniques are used to compensate the dispersion effects in the fiber. Three methods are prominent: the dispersion compensating fiber (DCF), fiber Bragg grating (FBG) and higher-order mode (HOM) fiber [Wan 2013]. 
Dispersion of single-mode fiber at a wavelength of $1550 \mathrm{~nm}$ can be compensated by specially designed fibers with negative and large dispersion coefficient $(D)$ at $1550 \mathrm{~nm}$. These types of fibers are known as dispersion compensating fibers (DCFs). Chirped fiber Bragg grating can be effectively used up to ultrahigh capacity $(100 \mathrm{GHz})$ long haul transmission systems for dispersion slope compensation. It is shown that the FBG can effectively compensate the dispersion slope while keeping proper in-line filtering [Chi 2007].

According to [Gra 2013], the effects of the second order dispersion parameter can be neglected provided the $3 d B$ linewidth $\delta \omega_{c}$ of the optical source satisfies the following expression:

$$
\delta \omega_{c} \approx \frac{1}{\sqrt{\left|\beta_{3}\right| L \Omega}}
$$

Therefore, we validate the choice of a BBS of $6 \mathrm{~nm}$ and the effects of $\beta_{3}$ can be neglected ( $\left.\boldsymbol{\beta}_{3}=\mathbf{1 . 5 7} * \mathbf{1 0}^{\mathbf{4}} \boldsymbol{p s} / \mathbf{n m}^{2} / \mathbf{k m}\right)$ so they will not be present in the experimental characterization.

\subsection{Experimental setup}

The transmission of OFDM signals over optical fiber was first proposed by [Chi 2017a, Chi 2017b] based on broadband optical sources for moderate transmission rates over WDM networks. A subcarrier multiplex transceiver based on dispersion-tolerant wide sources was presented in [Gra 2009]. This allowed the transmission of radio signals over optical fiber using incoherent slotted spectrum sources, such as ASE sources or LEDs in WDM networks.

Throughout this section we will present a WDM-OOFDM access network using a centralized optical transceiver based on a single optical broadband sliced source at the Central Office as a low-cost solution [Sek 2018] offering downlink and uplink signal transmission by using double sideband modulation and optical carrier reuse, as well as dynamic bandwidth allocation, multiple band selection and tunable OFDM band selection in reconfigurable networks at moderate bitrates.

\subsubsection{Generation and reception of OFDM signal}

The experimental design is based, at the electrical level, on off-line generation and reception. For this reason, digital signal processing will be carried out with Matlab, while digital-toanalogue and analogue-to-digital conversions will be carried out with AWG7122C (Arbitrary Waveform Generators) and DPO72004C (Digital Phosphor Oscilloscope) (Tektronix) equipment, respectively. Fig. 4.8 illustrates the experimental setup. 


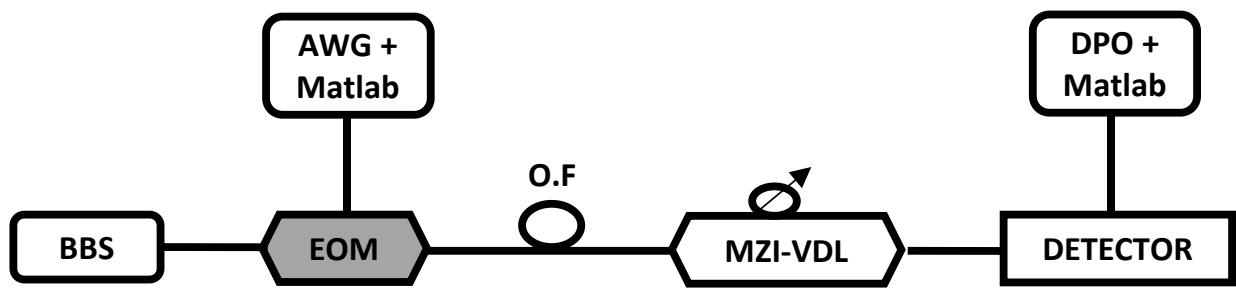

Fig. 4.8: OFDM-WDM network using broadband source. BBS: Broadband source (Optical). EOM: ElectroOptical Modulator. AWG: Arbitrary Waveform Generators. MZI-VDL: Mach-Zehnder Interferometer with Variable Delay Line. RTDO: Real Time Digital Oscilloscope.

The following figure shows the experimental system for transmitting OFDM signals in the electrical link.
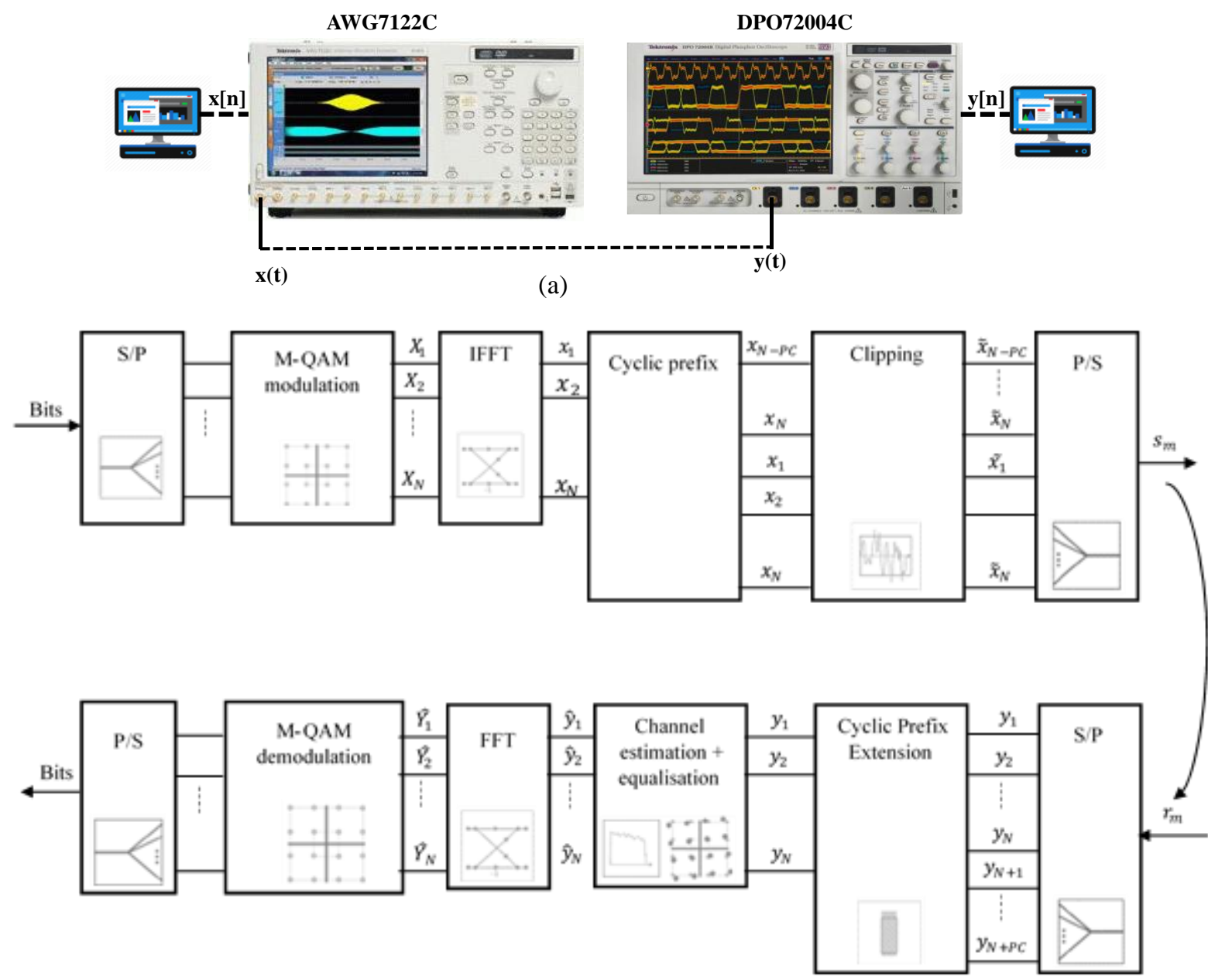

(b)

Fig. 4.9: (a) Experimental system for the transmission of OFDM signals in the electrical link, (b) Block diagram of generation and reception of an OFDM symbol.

The signal generator used in the experiments is an arbitrary waveform generator, AWG7122C, from Tektronix. This equipment is characterized by the ability to operate with two independent output channels sampled in the range $10 \mathrm{MS} / \mathrm{s}-12 \mathrm{GS} / \mathrm{s}$, or with one 
channel interleaved with samples in the range $12 \mathrm{GS} / \mathrm{s}-24 \mathrm{GS} / \mathrm{s}$; and the nominal electrical bandwidth of the transmitter is $7.5 \mathrm{GHz}$. It also includes a software for the generation of OFDM signals called RFXpress, allowing the configuration of a series of predefined parameters. However, in order to control all the signal parameters and to include non-standard blocks offering improved signal quality, we have chosen to design our own off-line signal processing scheme.

After optical receiver, the transmitted signal is recovered by using the DPO72004C Tektronix, which is a digital oscilloscope with 4 input channels offering a wide range of features and performance, allowing the reception of signals up to $20 \mathrm{GHz}$, with a maximum sampling rate of $100 \mathrm{GS} / \mathrm{s}$. Among its most outstanding features is the ability to view the received signal in the frequency domain. It also includes digital low-pass filters to eliminate unwanted frequency components from the received signal.

It also includes software (SignalVU) that allows the processing of the received signal. In the case of our experiment, again, we chose to design our own processing code for the received signal, in order to be able to configure the specific parameters of our received OFDM signal.

\subsubsection{OFDM signal parameters}

The OFDM multiplexing technique offers great flexibility in terms of configuring its parameters. The parameters of the OFDM signal that we are going to configure are the following:

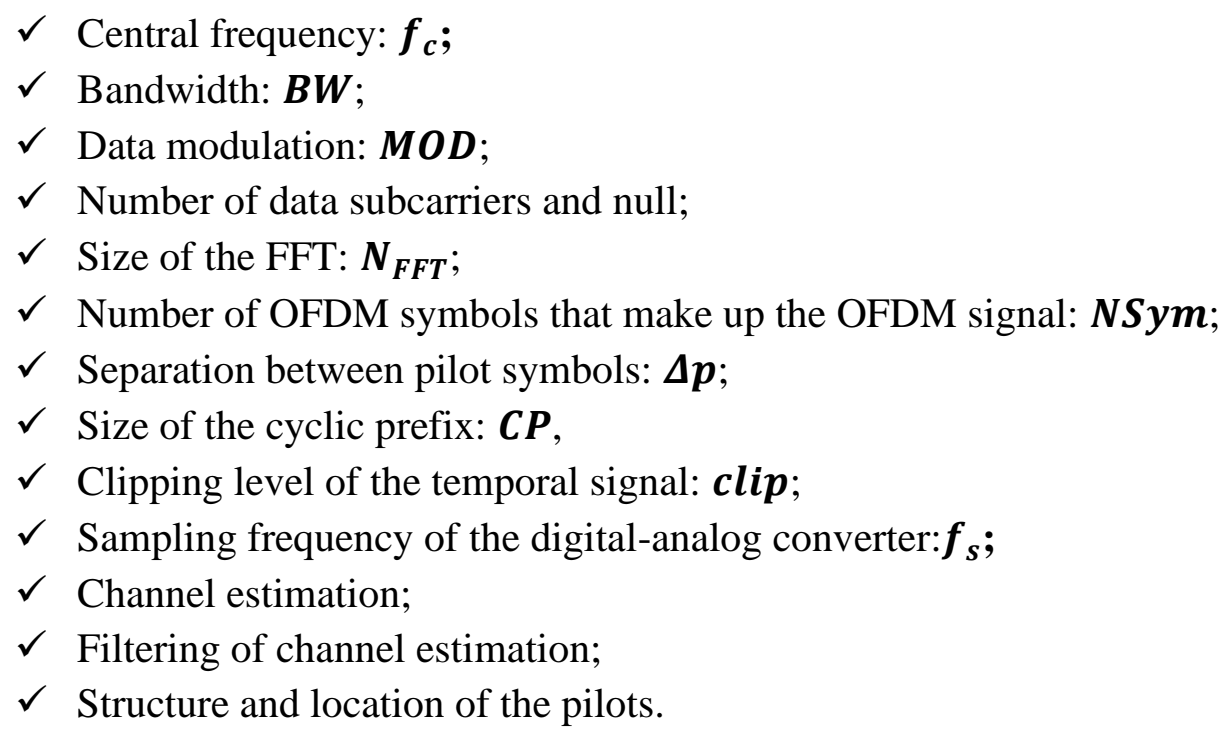

As explained above, a program has been developed under Matlab for the generation and analysis of the OFDM signal. Each block described in this section has its own routine and the whole calculation is executed by running a main program.

In an OFDM scheme, a high number of narrow-band orthogonal sub-carriers dividing the available bandwidth are transmitted in parallel. The interest of OFDM is mainly due to the way the system manages multipath interference at the receiver since multipath generates two undesired effects: frequency selective fading and inter-symbol interference (ISI). 


\subsubsection{Symbol generation}

The symbols to be transmitted can be pilots or data. The modulation used for the data is the QAM modulation, the number of levels of which is well determined. Once the $\boldsymbol{X}_{\boldsymbol{D}}[\boldsymbol{n}]$ data and the $\boldsymbol{X}_{\boldsymbol{P}}[\boldsymbol{n}]$ drivers have been generated, they give raise the following transmission matrix:

$$
X[n]= \begin{cases}X_{P}, & n=1,1+\Delta p, 2+\Delta p \\ X_{D}, & n=1,1+\Delta p, 2+\Delta p\end{cases}
$$

In an OFDM transmission system, the channel estimation and channel equalization blocks are fundamental for signal recovery at reception. The most popular technique invented by Cavers [25] for OFDM systems is pilot insertion. As is well known, pilot insertion in OFDM can be of the bock or comb type. In the block type pilot arrangement consists of allocating one or more time slots for pilot transmission, by using all subcarriers in those time slots. This approach is a suitable strategy for slow time-varying channels whereas in the comb type pilot arrangement a certain number of subcarriers are allocated to pilot symbols, continuously in time. This approach is a suitable strategy for non-frequency selective channels. The block type technique is the one used in our experimental part.

Eq. (4.16) gives rise to a block-type structure which is represented for $\Delta \boldsymbol{p}=\mathbf{4}$, and $\Delta \boldsymbol{p}=\mathbf{3}$ for a comb-type, as shows in Fig. 4.10 (a) and (b), respectively.
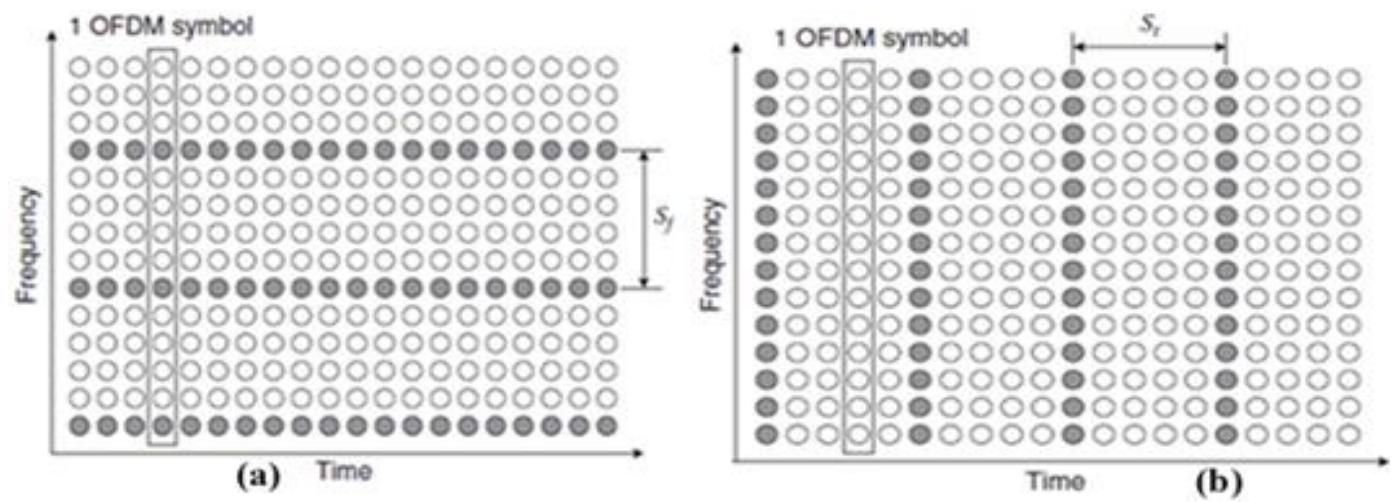

Fig. 4.10: Two pilot location patterns: (a) comb-type, (b) block-type.

Where $S_{t}$ denotes the period of the pilot symbols in time and $S_{f}$ the period of the pilot tones in frequency.

\subsubsection{Location and assignment of carriers}

From the values of the centre frequency, bandwidth, the size of the FFT, as well as the sampling frequency, we place the data carriers on the corresponding frequencies, leaving the carriers that carry no information to zero.

By discretizing the frequency, we establish the difference between the frequencies $\Delta \boldsymbol{f}$ as a quotient: 


$$
\Delta f=\frac{f_{s}}{N_{F F T}}
$$

Thus, the direct relationship between each frequency and the carrier number it presents is established from the following equation:

$$
f_{k}=k \cdot \Delta f, k=1,2, \ldots, N_{F F T}
$$

According to Nyquist's theorem, in order to avoid spectrum aliasing and to ensure that the discretized signal can be recovered, it is necessary that the maximum frequency of the signal doesn't exceed half the sampling frequency. Then, eq. (4.18) becomes:

$$
f_{k}=k . \Delta f, k=1,2, \ldots, \frac{N_{F F T}}{2}
$$

\subsubsection{Hermitian symmetry}

The Hermitian symmetry is used to obtain a real-valued time-domain signal. For $I Q$ modulators, both inputs must be real, the real part of the signal is transmitted in the $I$ branch and the imaginary part in the $Q$ branch. In the rest of the modulators, both with direct and external modulation, the input must be real. Therefore, the signal at the IFFT output must also be real. To meet this condition, the signal at the IFFT input must satisfy the hermitian symmetry and thus, the following conditions must be satisfied:

$$
I_{k}=\left\{\begin{array}{cc}
X_{k}, & k=1,2, \ldots, \frac{N}{2} \\
\bar{X}_{\frac{N}{2}-k+1}, & k=\frac{N}{2}+1, \frac{N}{2}+2, \ldots, N
\end{array}\right.
$$

Where: $I_{k}$ is the inputs of the IFFT block, $X_{k}$ is the data entered by the inputs and $N$ is the size of the FFT.

However, as the continuous and Nyquist components must be zero, the values $\boldsymbol{X}\left[\boldsymbol{f}_{\boldsymbol{k}}=\mathbf{0}\right]$ and $\boldsymbol{X}\left[\boldsymbol{f}_{\boldsymbol{k}}=\boldsymbol{f}_{\boldsymbol{s}} / 2\right]$ must be equal to zero. Combining the above conditions, we illustrate in table 4.3 the organization of the data at the input of the IFFT to respond to Hermitian symmetry.

Table 4.3: Organization of the data at the input of the IFFT to respond to Hermitian symmetry [Chi 2018].

\begin{tabular}{|c|c|c|c|c|c|c|c|c|}
\hline$I_{1}$ & $I_{2}$ & $\cdots$ & $I_{\frac{N}{2}-1}$ & $I_{\frac{N}{2}}$ & $I_{\frac{N}{2}+1}$ & $\cdots$ & $I_{N-2}$ & $I_{N-1}$ \\
\hline 0 & $X_{1}$ & $\cdots$ & $X_{\frac{N}{2}-2}$ & 0 & $\overline{\boldsymbol{X}}_{\frac{N}{2}-2}$ & $\cdots$ & $\overline{\boldsymbol{X}}_{\mathbf{2}}$ & $\overline{\boldsymbol{X}}_{\mathbf{1}}$ \\
\hline
\end{tabular}

On the $I_{k}$ symbol, the IFFT is applied so that if $\boldsymbol{x}_{\boldsymbol{k}}=\mathcal{F}^{-\mathbf{1}}\left\{\boldsymbol{I}_{\boldsymbol{k}}\right\}$, we can guarantee that $\boldsymbol{x}_{\boldsymbol{k}} \in \mathbb{R}$.

\subsubsection{The cyclic prefix}

A cyclic prefix $(\mathrm{CP})$ is created to prevent ISI when an OFDM signal is transmitted in a dispersive channel. The CP is essentially an identical copy of the last portion of the OFDM symbol appended before the OFDM symbol. In [Pel 1980] it is proposed for the first time the 
use of cyclic extensions so that those signals that are not cyclic are, at least, pseudo-cyclic. In this section we will focus on adding the final (initial) part of the signal at the beginning (end), which we will call the cyclic prefix.

However, the inclusion of the cyclic prefix makes the signal pseudo-cyclic and can be treated as if it were a cyclic signal. The received signal $r_{m}$ can be expressed by:

$$
\boldsymbol{r}_{\boldsymbol{m}}=\boldsymbol{s}_{\boldsymbol{m}} * \boldsymbol{h}_{\boldsymbol{m}}+\boldsymbol{n}_{\boldsymbol{m}}=\boldsymbol{s}_{\boldsymbol{m}} *\left(\boldsymbol{h}_{m}^{T X} * \boldsymbol{h}_{\boldsymbol{m}}^{c} * \boldsymbol{h}_{m}^{R X}\right)+\boldsymbol{n}_{m}, \quad \mathbf{0}<\boldsymbol{m}<\boldsymbol{N}-\mathbf{1}
$$

Where: $h_{m}^{T X}, h_{m}^{c}, h_{m}^{R X}$ represent the impulse responses of the transmitter, channel and receiver, respectively, $n_{m}$ is added as random noise and $s_{m}$ is the transmitted signal given by:

$$
\boldsymbol{s}_{\boldsymbol{m}}=\mathcal{F}^{-1}\left\{X_{k}\right\}
$$

Where: $\mathcal{F}^{-1}$ is the inverse Fourier transform, and $X_{k}$ is the information corresponding to the carrier $k$.

Since we are confronted with a pseudo-cyclic signal, this convolution becomes circular, so (4.21) becomes:

$$
\boldsymbol{r}_{\boldsymbol{m}}=\boldsymbol{s}_{\boldsymbol{m}} \circledast \boldsymbol{h}_{\boldsymbol{m}}+\boldsymbol{n}_{\boldsymbol{m}}, \quad \mathbf{0}<\boldsymbol{m}<\boldsymbol{N}-\mathbf{1}
$$

Fig. 4.11 illustrates an example of inserting a cyclic prefix.

(a)

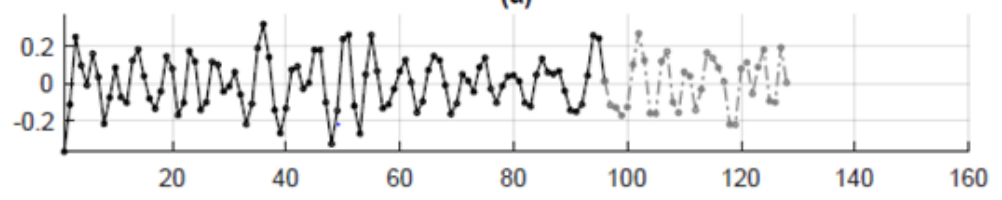

(b)

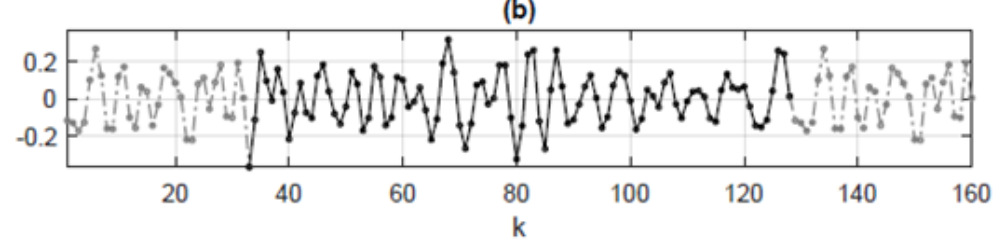

Fig. 4.11: An example of the insertion of a cyclic 25\% prefix on a 128 carriers signal: (a) original signal, (b) signal with cyclic prefix.

According to the Fig. 4.11 (b), it can be concluded that the signal at the output will have a large number of samples, which affects the transmission rate.

The cyclic prefix is included in the initial part of $X_{k}$. The parameter $C P$ determines the number of samples, so that $N_{C P}=C P . N_{F F T}$, where $N_{C P}$ is the number of samples to be reproduced. After applying the cyclic prefix, the symbol changes to:

$$
x_{C P}[k]=\left\{\begin{array}{cl}
x\left[N-N_{C P}+k\right], & k=1, \ldots, N_{C P} \\
x\left[k-N_{C P}\right], & k=N_{C P}+1, \ldots, N_{C P}+N
\end{array}\right.
$$




\subsubsection{Pre-emphasis filter}

The transmitter used in our experimental setup (AWG) doesn't show a flat amplitude response for the employed frequency range, so it is necessary to apply an $\boldsymbol{h}^{T X}(\boldsymbol{t})$ preemphasis filter that equalizes this response [Gao 2011, Cvi 2012]. The pre-emphasis filter is based on the transmission of different OFDM signals with data content in all carriers. From the spectra received in the electrical link, average values are obtained and smoothed by applying a low-pass filter. The pre-emphasis filter is obtained so that when multiplied in frequency over the received test signal, its response is as flat as possible, so that:

$$
\begin{cases}X^{R X}(f) \cdot H^{T X}(f)=1, & f \in\left[0, B W_{A W G}\right] \\ 0, & \text { rest }\end{cases}
$$

Where $X^{R X}(f)$ is the signal obtained by low-pass filtering and $B W_{A W G}$ is the RF bandwidth of the AWG.

Fig. 4.12 shows the effect of applying the pre-emphasis filter for a $16 Q A M-O F D M$ signal with $\boldsymbol{f}_{\boldsymbol{c}}=\mathbf{5 G H z}, \boldsymbol{B W}=\mathbf{0 . 7 5} \mathbf{G H z}$ (the bandwidth used throughout this section), $\boldsymbol{N}_{\boldsymbol{F F T}}=$ 2048 and $\boldsymbol{C P}=\mathbf{6}, \mathbf{2 5} \%$. From this figure it can be seen that the signal with pre-emphasis tries a priori to equalize the effects of the non-linear frequency response of the signal amplitude.

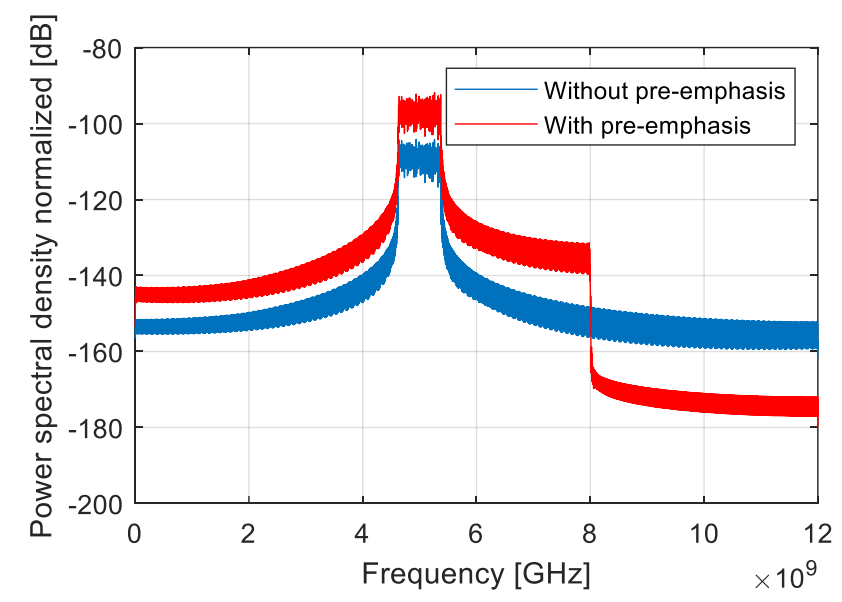

Fig. 4.12: Application of the pre-emphasis filter.

\subsubsection{Signal clipping}

Despite the advantages of OFDM, it has a major disadvantage related to the large amplitude variation of their generated signals, known as PAPR (Peak-to-Average Power Ratio). High PAPR occurs when several sinusoids are coherently added in an IFFT. The resulting peaks that exceed the operating range of a power amplifier (PA) cause a non-linear distortion that leads to a degradation of the bit error rate (BER) consequently, a degradation of the overall system performance [Hos 2016].

The value of the PAPR is defined as follows: 


$$
\boldsymbol{P A P R}=\frac{\max \left\{\left|s_{m}\right|^{2}\right\}}{\boldsymbol{E}\left\{\left|s_{m}\right|^{2}\right\}}
$$

Where $E\{$.$\} denotes the expectation operator.$

The PAPR in $d B$ is obtained by:

$$
P A P R_{d B}=10 \log _{10}(P A P R)
$$

However, there are various techniques for reducing PAPR, such as those based on clipping and filtering [Yos 2015] and Selective Mapping [Pam 2018]. The one we used in the experimental work is the clipping. The clipping is known as the simplest and most preferred technique, which consists of cancelling signal components that exceed an unchanged amplitude called Clip Level. In the case of our work, the threshold is set at $96 \%$, so the amplitudes above this threshold will be adjusted so that the signal will conform to the following equation:

$$
\widetilde{x}_{k}=\left\{\begin{aligned}
x_{k}, & \left|x_{k}\right|<\gamma \\
\gamma e^{j<x_{k}}, & \left|x_{k}\right|>\gamma
\end{aligned}\right.
$$

Where $\gamma$ is the threshold, and $\angle x_{k}$ denotes the phase of $x_{k}$.

Fig. 4.13 shows the signal clipped from the input signal for a $16-Q A M O F D M$ signal with $\mathrm{f}_{\mathrm{c}}=5 \mathrm{GHz}, \mathrm{BW}=0,75 \mathrm{GHz}, \mathrm{N}_{\mathrm{FFT}}=2048, \mathrm{CP}=6,25 \%$, and a pre-emphasis filter.

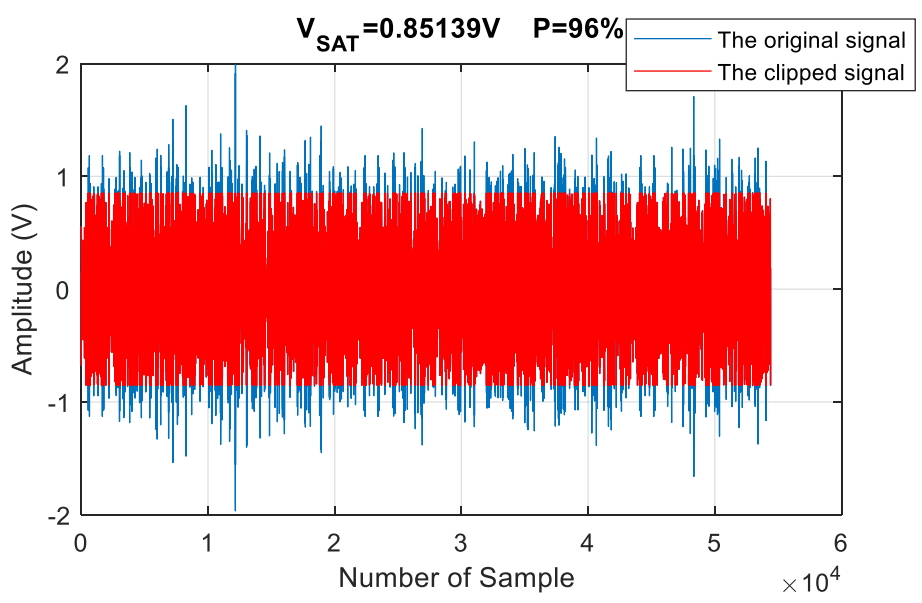

Fig. 4.13: Application of clipping of the temporal signal.

As soon as the signal has been clipped, all OFDM symbols are serialized to form the OFDM signal $x[n]$. This signal is loaded onto the AWG7122C signal generator, which is responsible for digital to analogue conversion and thus generates the $s(t)$ signal.

\subsubsection{Data rate of transmitted signal}

Usually, there are two transmission data rates related to OFDM signals: the first is the Net data rate $(D R)$ wich is the actual data rate (exact) and the second is the Raw data rate $(\widetilde{D R})$ (estimated) because is estimated as it considers a number of approximations. 
The actual data rate is calculated by:

$$
D R=\frac{B_{D}}{T}
$$

Where $B_{D}$ is bits of data transmitted and $T$ the time OFDM signal duration.

The amount of data bits transmitted can be deduced from the following equation:

$$
B_{D}=N_{D} K_{D} \log _{2}(M)
$$

Where $N_{D}$ is the symbols, $K_{D}$ is the subcarriers and $M$ is the number of symbols in the modulation.

In the complete OFDM signal, in addition to the data transport, other control symbols or null carriers are also included. The total number of samples $M_{T}$ is defined by:

$$
M_{T}=\left(N_{D}+N_{C P}\right)\left(N_{F F T}+N_{C P}\right)
$$

Where $N_{D}$ are the symbols dedicated to the data whereas $N_{C P}$ are dedicated to the cyclic prefix.

The digital-to-analog converter is defined by its sampling frequency $f_{S}$, so that each sample is transmitted every $T_{S}=1 / f_{S}$. Considering the number of samples $M_{T}$ in equation (4.31), the duration of the OFDM signal will have a duration of:

$$
T=T_{S}\left(N_{D}+N_{C P}\right)\left(N_{F F T}+N_{C P}\right)
$$

Therefore, from the equations (4.29), (4.30) and (4.30), the net data rate (DR) becomes:

$$
D R=\frac{N_{D} K_{D} \log _{2}(M)}{T_{S}\left(N_{D}+N_{P}\right)\left(N_{F F T}+N_{C P}\right)}
$$

Where $N_{P}$ is the number of pilot symbols.

Considering that the bandwidth of the signal can be expressed by $B W=f_{S} \frac{K_{D}}{N_{F F T}}$, and supposing that:

$$
\left\{\begin{array}{c}
N_{p} \ll N_{D} \rightarrow N_{p}+N_{D} \approx N_{D} \\
N_{C P} \ll N_{F F T} \rightarrow N_{F F T}+N_{C P} \approx N_{F F T}
\end{array}\right.
$$

So, the Raw data rate $\widetilde{(D R)}$ will be:

$$
\widetilde{D R} \approx B W \cdot \log _{2}(M)
$$

\subsubsection{Reception of the OFDM signal}

Once the $r(t)$ signal is received in the DPO72004C oscilloscope, where it is discretized, giving rise to the $r[n]$ signal. The processing of the received signal from its capture to the symbols is done via a Matlab code. Below, we will describe the steps to recover the transmitted symbols.

After receiving the signal, the first step is to resample the signal to the original sampling frequency fs, because the sampling frequencies of the transmitter and receiver can be different. For this, we use the resampling function $(x, p, q)$, where $x$ is the signal to be 
resampled, $p$ is the new sampling frequency, and $q$ is the sampling frequency of the input signal.

Our synchronization algorithm is based on obtaining the maximum of the correlation function between the transmitted signal and the received signal. Once the beginning of the received signal is detected and this signal is restructured to process it in matrix form, we eliminate the cyclic prefix. To do this, we again make a correlation between the first symbol received $y_{1}\left[k^{\prime}\right], k^{\prime}=1,2, \ldots, N, \ldots, N+N_{C P}$ and the first transmitted symbol $x_{1}[k], k=1,2, \ldots, N$. In this way, any error that might have occurred in the synchronization part is corrected. Once the cyclic prefix has been removed from the signal, the FFT is applied to recover the symbols.

The channel estimation block is responsible for obtaining the channel response. The technique is based on the reception of a series of symbols with known information. Using the least squares maximum probability estimator, the known symbols are compared with the received symbols. In the experimental part, block-type structures are used, so these symbols are the pilots. The latter are sent between the OFDM data symbols. In this way, the first symbol transmitted is a pilot, and each $\Delta p$ symbol will have a pilot, which will give a new frequency response.

From the experimental perspective, we have observed that this channel estimation can be improved, as better qualities of the received signal can be obtained. For this purpose, a lowpass filter for the frequency response has been introduced, the general characteristics of which can be found in [Mat 2014]. By working on normalized frequencies, we can design a generic filter that can be adjusted to proper value for each transmitted signal.

As the channel response represents an anticausal system, the transfer function $T F_{k}[n]$ will be a causal system, since we will average the current and previous frequency response to determine. Thus, the transfer function is:

$$
T F_{k}[n]=\left\{\begin{aligned}
H_{k}^{(1)}, & n=1,2, \ldots, \Delta p \\
\frac{1}{2}\left[H_{k}^{(P)}+H_{k}^{(P-1)},\right. & n>\Delta p
\end{aligned}\right.
$$

Where $\left.P=\frac{n}{\Delta p}\right\rceil$

Thus, the channel equalization which consists in applying the estimated transfer function to the received symbols. This transfer function modifies the amplitude and phase of the symbols according to the information contained in the pilot symbols.

\subsubsection{Quality of the OFDM signal}

The quality of OFDM transmission is measured using the Error Vector Magnitude (EVM) and the Bit Error Rate (BER) [Sha 2006, Chi 2018]. The evaluation of the quality of the received OFDM signal is carried out using a code in Matlab. The expression EVM relates the error power to the signal power, based on the expression:

$$
\operatorname{EVM}[\%]=\frac{\sum_{k=1}^{N}\left|R_{k}-T_{k}\right|^{2}}{\sum_{k=1}^{N}\left|T_{k}\right|^{2}}
$$


Where $R_{k}$ and $T_{k}$ are the received and transmitted symbols, respectively.

The bit error rate is obtained from the number of errors. In this way, we compare the transmitted data bits with those received. The BER limits are set at $10^{-3}$ and $3.8 * 10^{-3}$ for QPSK and 16-QAM modulations, respectively. The BER value is:

$$
B E R=\log _{10}\left(\frac{1}{M} \sum_{k=1}^{M} r_{k} \oplus t_{k}\right)
$$

Where $r_{k}$ and $t_{k}$ denotes the bits of received and transmitted data symbols, respectively, and $\bigoplus$ is the $X O R$ operator.

Another term that is the Signal-to-Noise Ratio (SNR) is a quantity commonly used to measure the quality of the signal perceived by the receiver. It is used in the case of analogue information and for digital transmissions. In addition, it is used to measure the impact of a component on the quality of the signal, since the components used in transmission systems, for example, are often characterized by the parameter "the noise factor", which makes it possible to assess their performance.

The signal-to-noise ratio $S / N$, or often SNR. It is calculated from the electrical signals by the following equation:

$$
S N R=\frac{P_{S}}{P_{B}}
$$

Where $P_{S}$ is the power of the signal in $W$, and $P_{B}$ is the power of the noise in $W$. Therefore, the SNR expressed in $d B$ is calculated by the expression:

$$
S N R_{d B}=10 \log _{10}(S N R)
$$

The BER can also be defined in terms of SNR as illustrated in the following equation [Han 2000]:

$$
B E R=\frac{2\left(1-\frac{1}{L}\right)}{\log _{2} L} Q\left[\sqrt{\left[\frac{3 \log _{2} L}{L^{2}-1}\right]\left[\frac{2 E_{s}}{N_{0} \log _{2} M}\right]}\right]
$$

Where $L$ and $\frac{E_{S}}{N_{0}}$ the number of levels in each dimension and the signal to noise ratio for the $M$-ary modulation system, respectively. $Q[$.$] is the gaussian co-error function.$

In order to establish relationship between BER and EVM, SNR can be expressed in terms of EVM as [Sha 2006]:

$$
S N R \approx \frac{1}{E V M^{2}}
$$

Combining equations (4.41) and (4.42), we can now relate the BER directly with the EVM as follows:

$$
B E R \approx \frac{2\left(1-\frac{1}{L}\right)}{\log _{2} L} Q\left[\sqrt{\left[\frac{3 \log _{2} L}{L^{2}-1}\right] \frac{2}{E V M_{R M S}^{2} \log _{2} M}}\right]
$$




\subsubsection{Optical components}

In this section, we will describe the characteristics of the equipment used in our experimental setup.

\subsubsection{The Broadband source (BBS)}

NP Photonics C\&L Band ASE Source is a high performance, high power, low cost and reliable incoherent light source. This high-power source produces a broadband output spectrum (Fig. 4.5(a)) of Amplified Spontaneous Emission (ASE) ranging from 1525 to $1610 \mathrm{~nm}$. It offers high levels of power, spectral, and temperature stability for the most demanding ASE applications. The main characteristics used in our experimental setup are presented in Table 4.4 .

Table. 4.4: The characteristics of BBS NP Photonics C\&L Band ASE Source.

\begin{tabular}{|l|l|}
\hline \multicolumn{1}{|c|}{ Parameters } & \multicolumn{1}{c|}{ Value } \\
\hline Wavelength Range & $1550 \mathrm{~nm}$ \\
\hline Output Power & $19.5 \mathrm{dBm}$ \\
\hline Output Power Stability $(\mathrm{dB})(1 \mathrm{hr})$ at fixed ${ }^{\circ} \mathrm{C}$ & $< \pm 0.01$ \\
\hline Minimum spectral power density & $-12 \mathrm{dBm} / \mathrm{nm}$ \\
\hline Optical Bandwidth @10 $\mathrm{dB}$ point & $>85 \mathrm{~nm}$ \\
\hline Output Polarization (Degree of Polarization) & $<5 \%$ \\
\hline Package Dimension & $310.12 \times 257 \times 103 \mathrm{~mm}$ \\
\hline
\end{tabular}

\subsubsection{Multiport Tunable Optical Filter (MTOF)}

The MTOF of the Finisar WaveShaper $4000 S$ family provide extremely fine control of filter amplitude and phase characteristics across the C-Band, L-Band or $\mathrm{C}+\mathrm{L}$ bands. It allows the generation of arbitrary, complex filters as well as control of filter bandwidth and center frequency with $1 \mathrm{GHz}$ resolution. They contain no moving parts and so provide extremely high reliability for both laboratory and production test applications.

The main optical characteristics of the MTOF are presented in Table 4.5.

Table 4.5: The optical characteristics of MTOF (Finisar WaveShaper 4000S).

\begin{tabular}{|l|l|}
\hline \multicolumn{1}{|c|}{ Parameters } & \multicolumn{1}{c|}{ Value } \\
\hline Wavelength Range & 1527.4 to $1600.8 \mathrm{~nm}$ \\
\hline Filter Bandwidth & $0.08-38.4 \mathrm{~nm}$ \\
\hline Frequency setting resolution & $\pm 1 \mathrm{GHz}( \pm 8 \mathrm{pm})$ \\
\hline Bandwidth setting resolution & $\pm 1 \mathrm{GHz}( \pm 8 \mathrm{pm})$ \\
\hline Attenuation control range & $0-35 \mathrm{~dB}$ \\
\hline Attenuation setting resolution & $0.01 \mathrm{~dB}$ \\
\hline Insertion loss & $4.5 \mathrm{~dB}$ \\
\hline Max total optical input power & $+27 \mathrm{dBm}$ \\
\hline Max optical power per 50GHz channel & $+13 \mathrm{dBm}$ \\
\hline
\end{tabular}




\subsubsection{Electro-Optic Modulator (EOM)}

The EOM used in our experimental setup is of the Avanex type. These main features are summarized in Table 4.6.

Table 4.6: The characteristics of EOM Avanex.

\begin{tabular}{|l|l|}
\hline \multicolumn{1}{|c|}{ Parameters } & \multicolumn{1}{c|}{ Value } \\
\hline$V_{p}$ bias Electrode @ $1 \mathrm{KHz}$ & $4.7 \mathrm{~V}$ \\
\hline$V_{p}$ RF Electrode @ $1 \mathrm{KHz}$ & $3.5 \mathrm{~V}$ \\
\hline Extinction Ratio & $29.4 \mathrm{~dB}$ \\
\hline Bandwidth $-3 \mathrm{~dB}$ & $12.5 \mathrm{GHz}$ \\
\hline Optical insertion loss & $3.1 \mathrm{~dB}$ \\
\hline
\end{tabular}

\subsubsection{Polarization controller}

Polarization Controller is a basic component in fiber optics experiments. FIBERPRO's PC1000 series provides smooth and convenient control of the state of polarization. Threepaddle polarization controllers manipulate the output polarization state for tight-buffered fiber. It uses mechanical bending and rotation of the fiber to produce stress-induced birefringence, which in turn produces changes in the output polarization. The stress-induced birefringence can be adjusted continuously, allowing any arbitrary input polarization state to be converted to any desired output polarization state.

General Photonics manual Variable Optical Delay Line provides precision optical path variation of more than $18 \mathrm{~cm}$, which corresponds to $600 \mathrm{ps}$ maximum delay in time domain. The delay time can be manually adjusted from 0 to $600 p s$ continuously based on a sliding delay indicator on the package.

The main characteristics of the VDL are presented in Table 4.7.

Table 4.7: The specifications of the VDL General Photonics.

\begin{tabular}{|l|l|}
\hline \multicolumn{1}{|c|}{ Parameters } & \multicolumn{1}{c|}{ Value } \\
\hline Insertion loss & $1 d B$ (without connectors) \\
\hline Wavelength & $1270 \sim 1650 \mathrm{~nm}$ \\
\hline Fiber type & $S M F-28$ standard \\
\cline { 2 - 2 } & $\begin{array}{l}\text { Polarization maintaining fiber: PM Panda fiber } \\
\text { standard }\end{array}$ \\
\hline Extinction Ratio & $>18 \mathrm{~dB}$ for PM fiber \\
\hline Maximum power & $300 \mathrm{~mW}$ min \\
\hline
\end{tabular}

\subsubsection{Receiver}

The balanced photodetector $\mathrm{u} 2 \mathrm{t}$ photonics BPDV2020R is based on the principle of converting two optical input signals from the optical domain into the electrical one and providing the difference of the photocurrents at the RF output. The $3 d B$ electrical $\mathrm{BW}$ exceeds $40 \mathrm{GHz}$. 
Table 4.8 shows the specifications of the receiver employed in our setup.

Table 4.8: The specifications of the balanced photodetector $\mathrm{u} 2 \mathrm{t}$ photonics BPDV2020R.

\begin{tabular}{|l|l|}
\hline \multicolumn{1}{|c|}{ Parameters } & \multicolumn{1}{c|}{ Input 1 } \\
\hline Dark current (nA) & -0.9 \\
\hline Responsivity (A/W) & 0.55 \\
\hline Pulse width $(\mathrm{ps})$ & 9.5 \\
\hline Cut-off-frequency $(\mathrm{GHz})$ & 43.1 \\
\hline
\end{tabular}

\subsubsection{OOFDM-WDM experimental network}

The proposed network employs IM-DD for both the DL and UL, allowing an Optical Line Terminal (OLT) transceiver at the Central Office (CO) based on a single broadband source which is sliced to generate optical carriers feeding different ONUs, and contains a receiver based on a PIN photodiode (PD) for the UL signal. Simple ONU transceivers include the detection stage for the DL signal detection and an electro-optic modulator (EOM) to remodulate the DL signal with the UL OFDM signal. DL and UL signals employ different frequency bands, which are selected by the proper configuration of both detection stages. The detection stage required for optical OFDM signal transmission using optical broadband sources is based on the use of a tunable Mach-Zehnder interferometer (TMZI) and a PIN photodiode. Fig. 4.14 presents the scheme of the experimental setup.

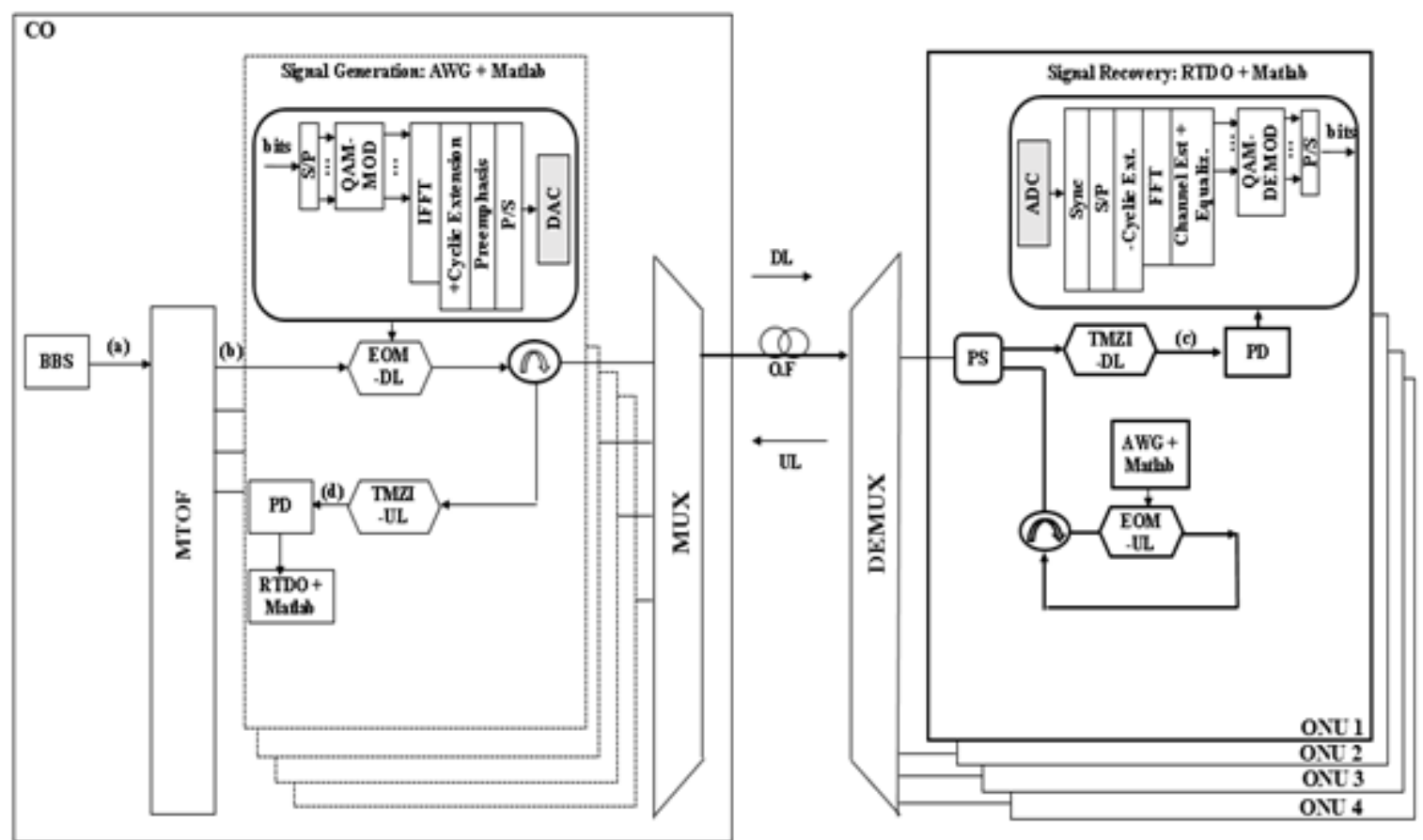

Fig. 4.14: Centralized bidirectional OOFDM-WDM network using broadband source. BBS: BroadBand Source (Optical), MTOF: Multiport Tunable Optical Filter, EOM: Electro-Optical Modulator, MUX: Multiplexer, DEMUX: Demultiplexer, PS: Passive splitter, TMZI: Tunable Mach-Zehnder Interferometers, DL: Downlink, UL: Uplink, OF: Optical Fiber, PD: Photodetector, AWG: Arbitrary Waveform Generator (RF), RTDO: Real Time Digital Oscilloscope, CO: Central Office, ONU: Optical Network Unit. 
The optical carrier is generated by a broadband source (BBS) at the OLT with $19.5 \mathrm{dBm}$ power. The broadband optical spectrum is optically sliced by using a multiport tunable optical filter (MTOF) which provides coarse wavelength division multiplexing (CWDM) and allows the selection of the central frequency and the optical bandwidth of the signal supporting each ONU. In this case, we employed an optical channel controller (Finisar WaveShaper 4000S), which allows channel selection with rectangular channel widths in the range of $0.08-$ $38.4 \mathrm{~nm}$.

Optical signals generated at OLT are double sideband (DSB) modulated by an OFDM signal in a single-drive electro-optic modulator (EOM, AVANEX) and multiplexed via a CWDM before transmission over fiber. After transmission, the optical OFDM signals are demultiplexed and, at each ONU, the received signal is split with a passive splitter (PS) where one portion of the signal is detected and the other one is re-modulated with the UL signal by means of an EOM. The block diagram of the OFDM signal generation can be seen in Fig. 4.14, whose implementation has been described in the previous section. The electrical multiband OFDM signal is generated offline in Matlab and loaded into an Arbitrary Waveform Generator (AWG). An IFFT size of 2048 with $Q P S K$ or $16-Q A M$ data-bearing subcarriers satisfying Hermitian symmetry. The occupied band is centered at a frequency up to $6 \mathrm{GHz}$, with a 700 to $1500 \mathrm{MHz}$-bandwidth in different experiments with a raw data rate below $2 \mathrm{~Gb} / \mathrm{s}$. A cyclic prefix of $6.25 \%$ of the symbol is applied, the data are loaded onto the digital-analog converter of the arbitrary wave generator (AWG7122C, Tektronix), sampled at $24 \mathrm{GS} / \mathrm{s}$, and equalization in detection process is performed by the inclusion of block-type equispaced pilot pattern of $10 \%$. A pre-emphasis filter is also applied to overcome the nonflat response of the electrical transmitter. At each ONU, prior to photodetection, there is a TMZI composed by two 50: 50 optical couplers and a variable delay line (VDL) in one of the arms. As a result of the insertion of this component, the bandwidth limitation arisen from chromatic dispersion in broadband signals is overcome and a bandpass window is generated in the RF transfer function of the overall system.

Just after the TMZI, the signal is photodetected. The resulting electrical signal is sampled at $50 G S / s$ on a real time digital oscilloscope (RTDO, DPO72004C, Tektronix). The captured samples are synchronized and parallelized for processing in the regular OFDM receiver blocks (Fig.4.14). After removing the cyclic prefix, the FFT is applied. Block-type structure pilots are used for channel estimation, from which equalization is performed. Finally, data are demodulated and the transmitted bit stream is recovered after the serialization process.

Fig. 4.15 shows a picture of the experimental setup in the laboratory. The specifications of the required equipment in this experimental setup is presented in table 4.9. 


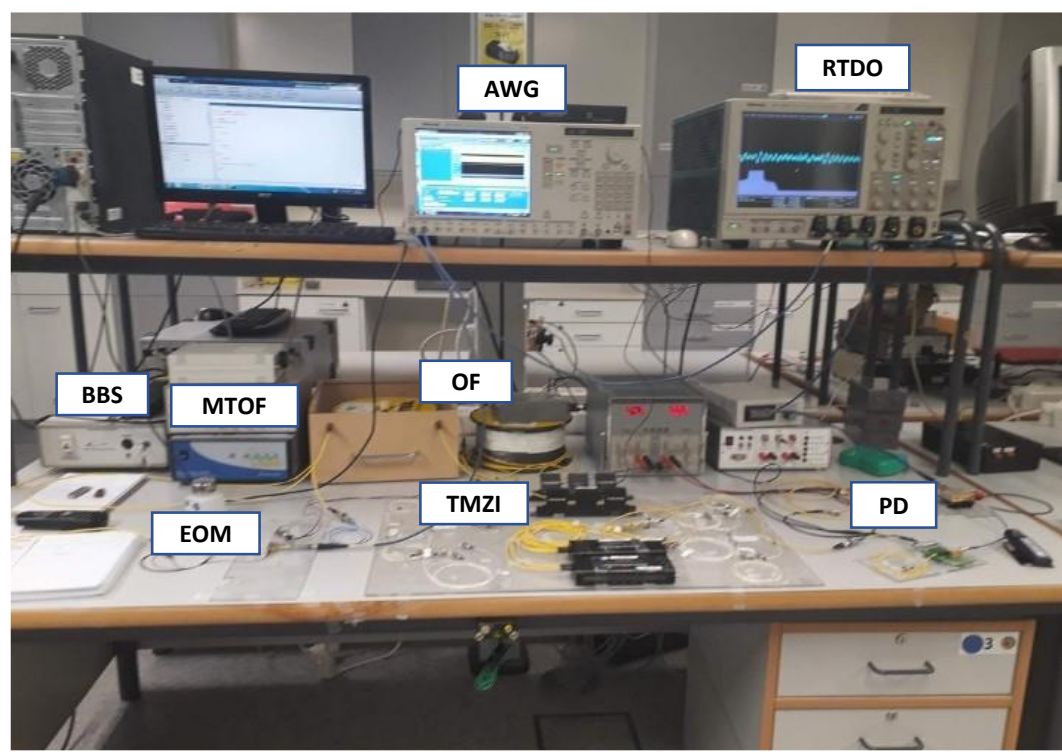

Fig. 4.15: Experimental setup (photograph).

Table. 4.9: Specifications of the equipment in the OOFDM-WDM proposed network.

\begin{tabular}{|c|c|c|}
\hline & Model & Characteristics \\
\hline AWG & AWG7122C, Tektronix & Sampling rate $=24 \mathrm{GS} / \mathrm{s}$ \\
\hline BBS & NP Photonics C\&L Band ASE Source & $\begin{array}{l}\text { Optical Power }=19.5 \mathrm{dBm} \\
\text { Wav. Range }=1525-1610 \mathrm{~nm}\end{array}$ \\
\hline MTOF & Finisar WaveShaper 4000S & $\begin{array}{l}\text { Wav.Range }=1527-1568 \mathrm{~nm} \\
\text { Steps of } 77 \mathrm{pm} \\
\text { Roll-off value of } 0.06 \mathrm{~dB} / \mathrm{pm}\end{array}$ \\
\hline EOM & Avanex & $\begin{array}{l}\mathrm{Vp}=5 \mathrm{~V} \\
\text { Insertion } \operatorname{loss}(\mathrm{Vp})=3.5 \mathrm{~dB}\end{array}$ \\
\hline PS & Fibercore & Insertion losses $=0.5 \mathrm{~dB}$ \\
\hline RTDO & DPO72004C, Tektronix & Sampling rate $=50 \mathrm{GS} / \mathrm{s}$ \\
\hline TMZI & $\begin{array}{l}\text { Fiberco 50:50 couplers } \\
\text { General Photonics Variable Optical } \\
\text { Delay Line (VDL) } \\
\text { Fiberpro polarization controller }\end{array}$ & $\begin{array}{l}\text { Total Insertion Losses }=4 \mathrm{~dB} \\
\text { Max. delay between TMZI arms }=300 \mathrm{ps}\end{array}$ \\
\hline PD & u2t photonics BPDV2020R & $\begin{array}{l}\text { Bandwidth }=40 \mathrm{GHz} \\
\text { Responsivity=0.55 A/W }\end{array}$ \\
\hline
\end{tabular}

The remodulated UL signals at different ONUs are launched back to the network through an optical circulator, combined and transmitted over fiber. At the OLT, the received signal is demultiplexed, and a circulator provides de UL signal separation which will be detected after the TMZI stage configured to transmit the UL operating band. The proposed system allows flexible selection of the OFDM subcarriers band employed for each ONU, both for DL and UL, and also multiband selection is allowed in TMZI multistage configuration, as will be explained below.

The main advantages of our proposed system is the use of a single broadband low cost source to feed multiple ONUs to transmit OFDM signals by IM-DD double sideband modulation for both uplink and downlink, also including carrier reuse for uplink transmission and colorless 
detection both at OLT and ONU. Optical channels assignment to each ONU and operating OFDM bands can be dynamically changed according to the network conditions and current ONU demands.

\subsection{Experimental results and applications}

Full characterization of the network described above with a $10 \mathrm{~km}$ standard single mode fiber link will be done in order to assess its viability as a low-cost solution for transmitting optical OFDM signals at moderate bitrates. Flexibility of the proposed network architecture in terms of frequency bands and signal bandwidths for different ONUs will be also shown in a reconfigurable network scenario.

\subsubsection{Carrier reuse for bidirectional transmission}

The Fig. 4.6(b) presents the sliced optical spectrum corresponding to the signal carrier assigned to ONU 1 is $6 \mathrm{~nm}$ wideband. The TMZI is configured at this ONU with a differential delay of $\Delta \tau=6.28 p s$, which, according to eq. (4.7), leads to a bandpass transmission window centered at $5 \mathrm{GHz}$, and shows electrical insertion losses of $10.5 \mathrm{~dB}$.

The electrical amplitude response of the overall downlink system is measured and depicted in Fig. 4.16 (a). Fig. 4.16 (b) shows the uplink electrical response for the overall system when the optical carrier, generated at the $\mathrm{CO}$, is reused at the ONU and modulated by an uplink $450 \mathrm{Mb} / \mathrm{s}$ QPSK OFDM signal. In this case, the TMZI at the CO, prior to detection, is configured with a delay $\Delta \tau=4.4 \mathrm{ps}$, which corresponds to a $3.5 \mathrm{GHz}$ transmission band (see eq. (4.7) with $3 \mathrm{~dB}$ bandwidth of $0.9 \mathrm{GHz}$ and electrical insertion losses of $9 \mathrm{~dB}$.

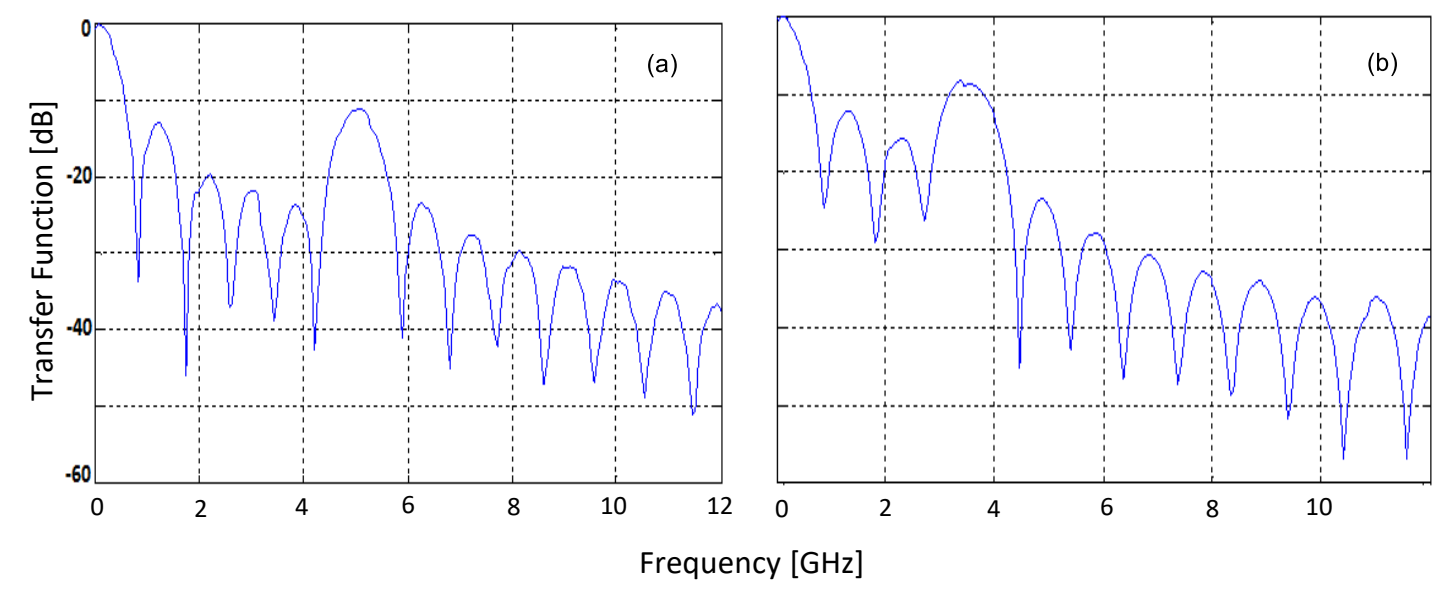

Fig. 4.16: Electrical transfer function of the proposed system. (a) Downlink, (b) Uplink.

Fig. 4.17 shows the electrical power spectral density of the received UL (Fig. 4.17(d)) also shows the modulating OFDM signal, confirming the effective transmission of the $750 \mathrm{MHz}$ OFDM bandwidth upstream signal band centered at 3.5 $\mathrm{GHz}$. A residual transmitted band of 
$5 \mathrm{GHz}$ at the UL detector can also be seen in Fig. 4.17 (b) where a rejection ratio greater than $14 d B$ is obtained.

This confirms the effective transmission of the downstream $750 \mathrm{MHz}$ bandwidth OFDM downlink signal band centered at $5 \mathrm{GHz}$ with $750 \mathrm{MHz}$ bandwidth and $0.9 \mathrm{~Gb} / \mathrm{s}$, and the effective transmission of the upstream $750 \mathrm{MHz}$ bandwidth OFDM signal band centered at 3.5 GHz, as shown in Fig. 4.17.

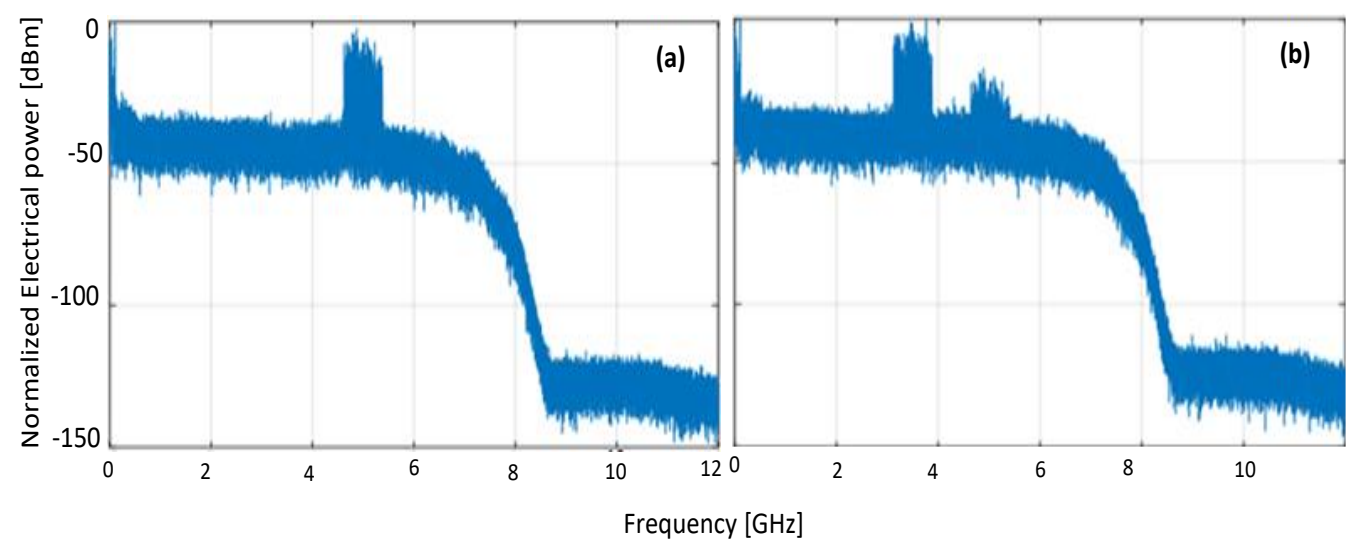

Fig. 4.17: Power spectral density of received electrical signal: (a) Downstream, (b) Upstream.

Fig. 4.18 and Fig. 4.19 show the constellations of the electrical back-to-back (EB2B) and received 16-QAM and QPSK signals corresponding to DL and UL, respectively. In both cases, EVM was measured for different OFDM subcarriers (SC) and also per symbol, with an average value below $30 \%$ (18.97\% for 16-QAM DL and $13.75 \%$ for QPSK UL).
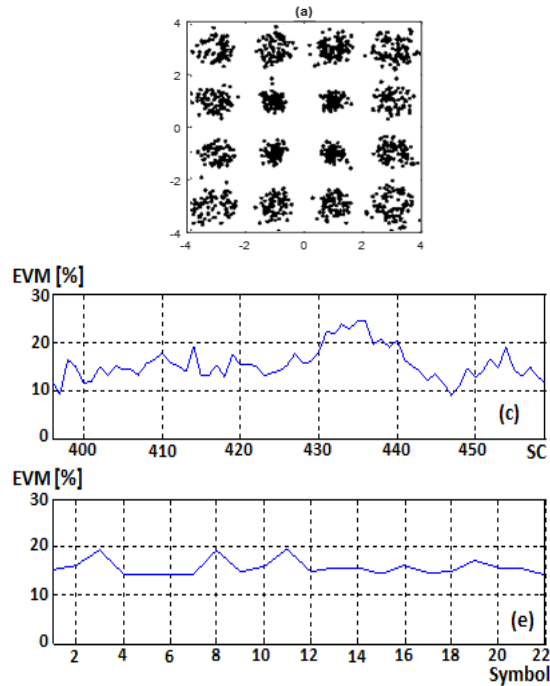
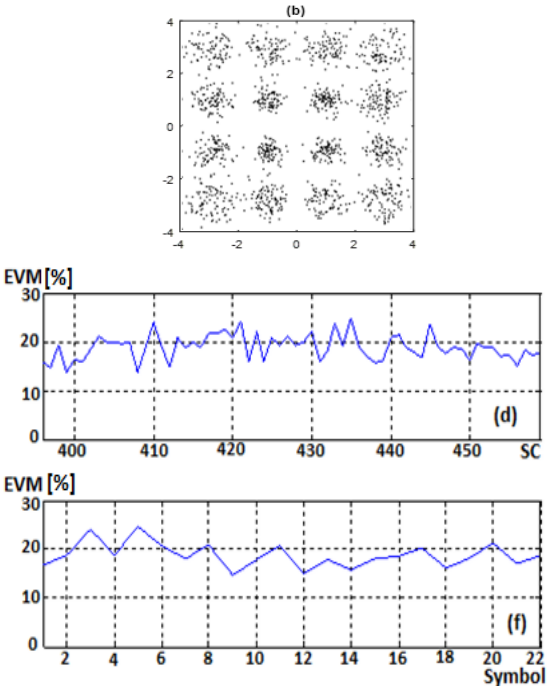

Fig. 4.18: Downstream transmission: $750 \mathrm{MHz}$ 16-QAM OFDM signal carried at $5 \mathrm{GHz}$. EB2B results: (a) Constellation, (c) EVM per subcarrier, (e) EVM per symbol. After $10 \mathrm{~km}$ fiber transmission link: (b) Constellation, (d) EVM per subcarrier, (f) EVM per symbol. 

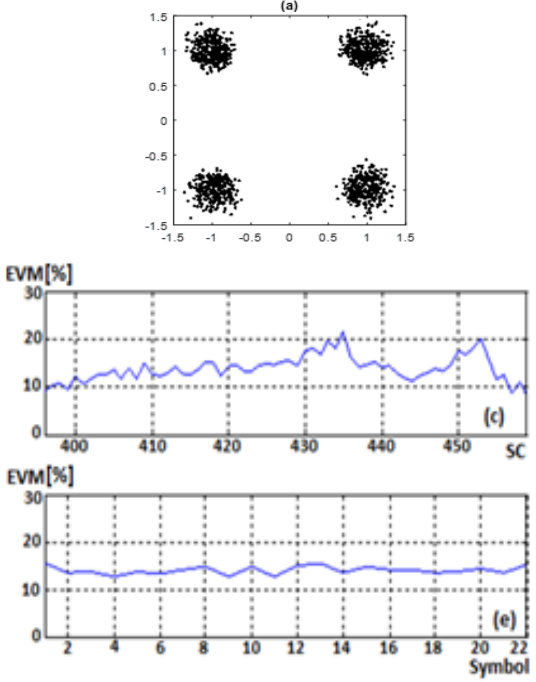
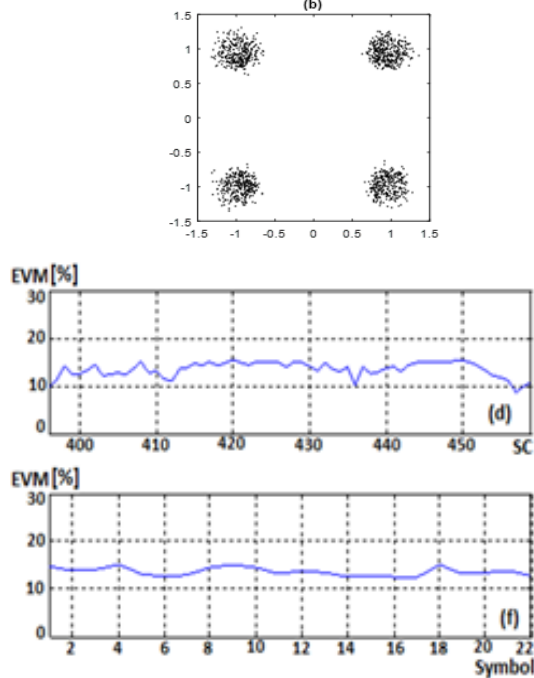

Fig. 4.19: Upstream transmission: $750 \mathrm{MHz}$ QPSK OFDM signal carried at 3.5G $\mathrm{Hz}$. EB2B results: (a) Constellation, (c) EVM per subcarrier, (e) EVM per symbol. After $10 \mathrm{~km}$ fiber transmission uplink: (b) Constellation, (d) EVM per subcarrier, (f) EVM per symbol.

\subsubsection{Dynamic bandwidth allocation}

According to the rectangular profile of the MTOF output (as shown in Fig. 4.5(b)), the $3 d B$ electrical bandwidth $\delta \mathrm{f}_{3 \mathrm{~dB}}$ of the RF bandwidth in our system can easily be obtained by eq. (4.8).

In this experiment, a $2.66 \mathrm{GHz}$ bandwidth 16-QAM OFDM signal centered at $5 \mathrm{GHz}$ is generated as depicted in Fig. 4.20, where the power spectral density of the EB2B as well as its EVM measurement per subcarrier and symbol is plotted.
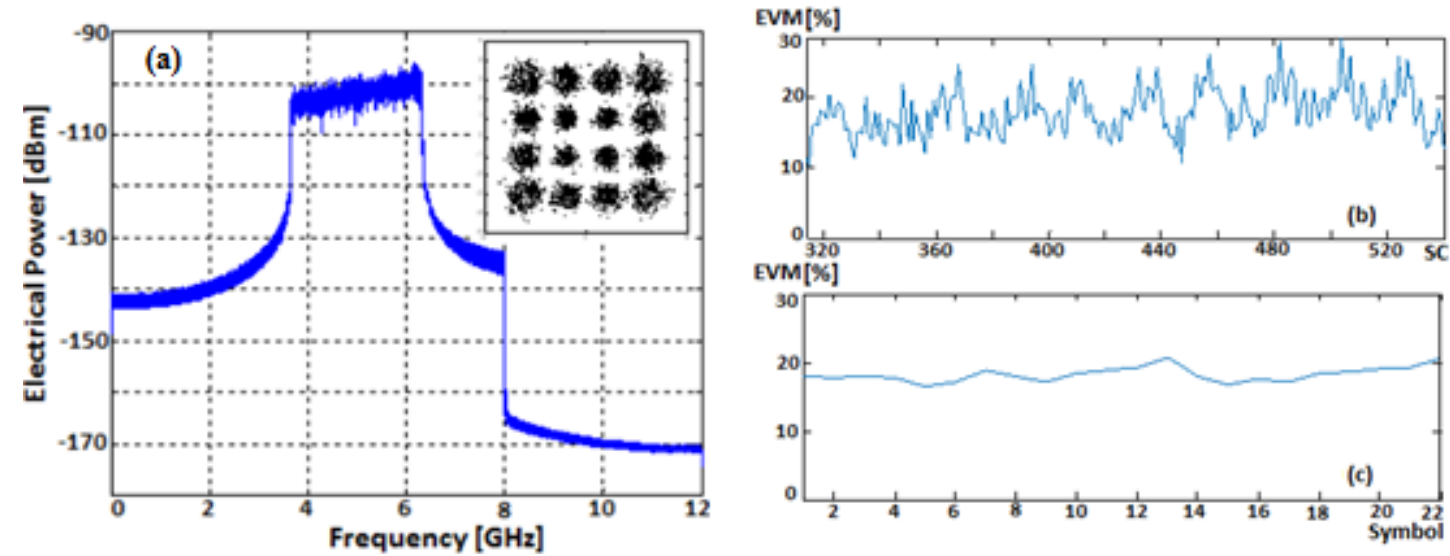

Fig. 4.20: (a) EB2B power spectral density for $B W=2.66 \mathrm{GHz}$ (inset: constellation), (b) EVM per subcarrier, (c) EVM per symbol.

Electrical transfer functions were measured at $5 \mathrm{GHz}$ with different optical bandwidths over a $10 \mathrm{~km}$ fiber link, as shown in Figs. 4.21 and 4.22. The effective $3 \mathrm{~dB}$ electrical bandwidth of 
the transmitted signal is 1.568 and $0.664 \mathrm{GHz}$ when the optical bandwidth is set as 3.9 and $8 \mathrm{~nm}$, respectively, and accordingly, the electrical spectrum of the transmitted signal is depicted with the minimum EVM value obtained at the center of the transmitted band, shown as the inset, which is lower than $35 \%$.

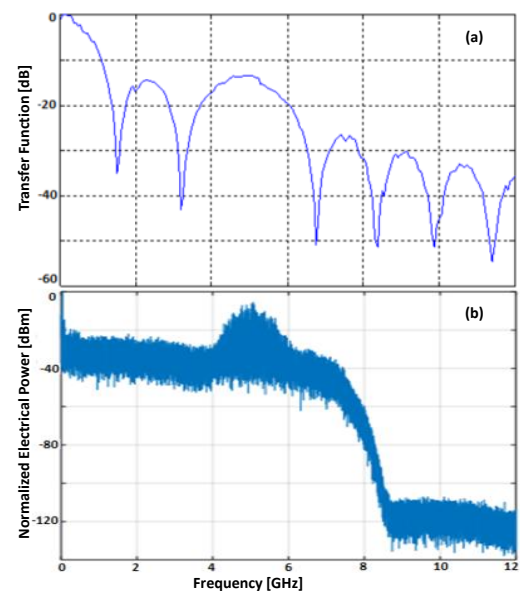

Fig. 4.21: Transmission system response when optical carrier bandwidth is 3.9nm: (a) Electrical transfer function, (b) Power spectral density of recovered electrical signal when modulating signal is the one depicted in Fig. 4.19.

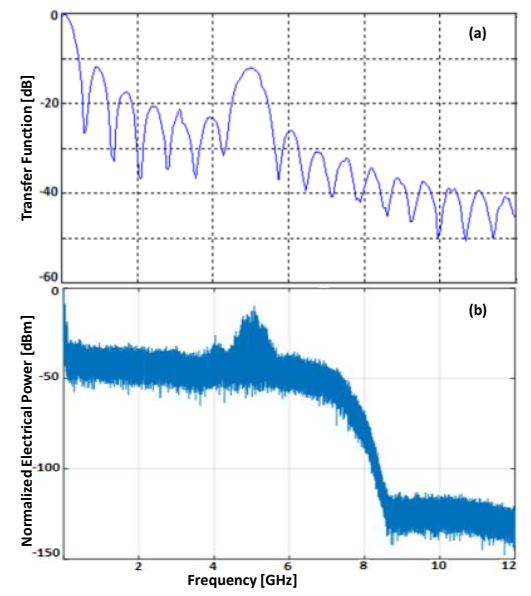

Fig. 4.22: Transmission system response when optical carrier bandwidth is 8nm (modulating signal is depicted in Fig. 4.20): (a) Electrical transfer function, (b) Power spectral density of recovered electrical signal.

Different linewidths of the optical source leads to different electrical bandwidths. Therefore, larger electrical bandwidths result in a higher number of subcarriers, and therefore higher bit rate can be transmitted, as shown in Table 4.10. 
Table 4.10: Dynamic bandwidth allocation: effective bitrates for different linewidths of the optical source.

\begin{tabular}{|l|l|l|l|}
\hline$\delta \lambda_{3 \mathrm{~dB}}(\mathrm{~nm})$ & \multirow{2}{*}{ Bandwidth $(\mathrm{GHz})$} & \multicolumn{2}{|c|}{ Effective bitrate $(\mathrm{Gb} / \mathrm{s})$} \\
\cline { 3 - 4 } & & QPSK & $16-\mathrm{QAM}$ \\
\hline 3.9 & 1.47 & 0.882 & 1.764 \\
\hline 6 & 0.95 & 0.57 & 1.14 \\
\hline 8 & 0.71 & 0.426 & 0.852 \\
\hline 10 & 0.57 & 0.342 & 0.684 \\
\hline 15 & 0.38 & 0.228 & 0.456 \\
\hline
\end{tabular}

Therefore, the proposed network shows moderate bitrates performance but enables bidirectional transmission as well as dynamic bandwidth allocation by using a low-cost solution based on a broadband source, as demonstrated above.

\subsubsection{Multiple users in reconfigurable networks}

The MTOF is a flexible component which dynamically assigns part of the optical spectrum emitted by the broadband optical source and provided the configuration at the TMZI is adjusted according to the dispersion term of the fiber link $\left(\beta_{2} L\right)$ in eq. (4.10). In the context of reconfigurable multi-users networks, the proposed scheme is depicted in Fig. 4.23.

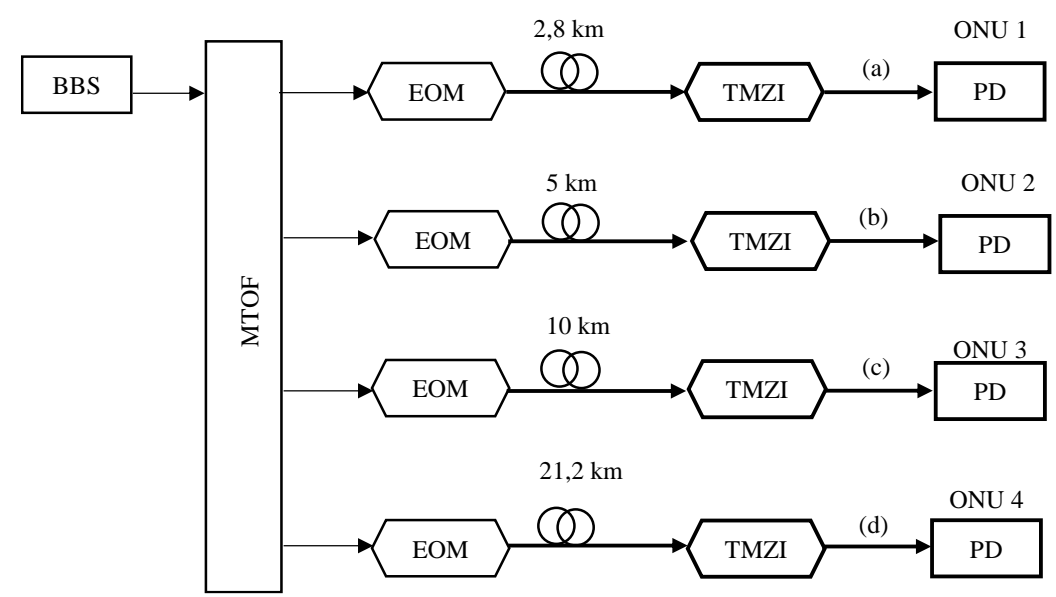

Fig. 4.23: The schematic for reconfigurable multi-users networks.

The OOFDM signal transmission system based on broadband source and MZI-VDL is also evaluated on a WDM network, as shown in Fig. 4.23, to assess its feasibility. In this case, four WDM channels are employed in the network, where the OFDM signal is centered at $5 \mathrm{GHz}$ and the length of the links connecting the CO to different ONUs is $2.8,5,10$ and $21.2 \mathrm{~km}$, respectively, but using optical broadband sources with $6 \mathrm{~nm}$ linewidth, but centred at 1549 , 1552, 1555 and $1558 \mathrm{~nm}$, respectively. 


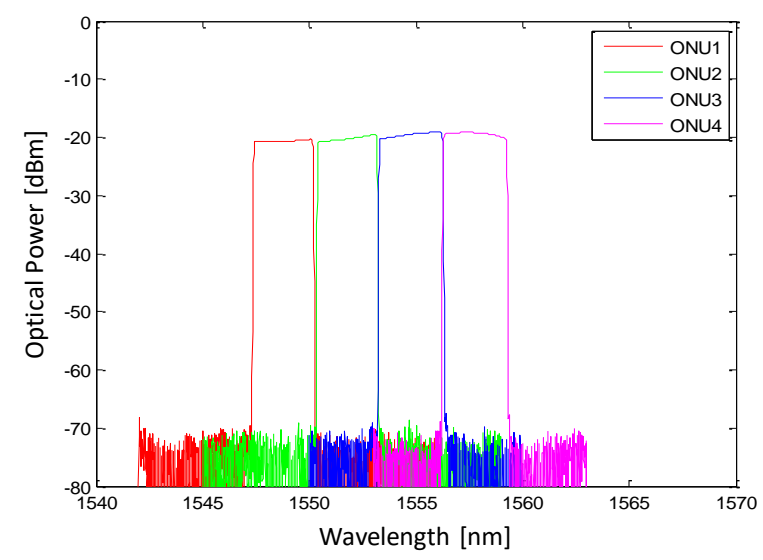

Fig. 4.24: The spectral bands assigned to four ONUs in the WDM network.

Optical spectra measured at each ONU are illustrated in Fig. 4.25.

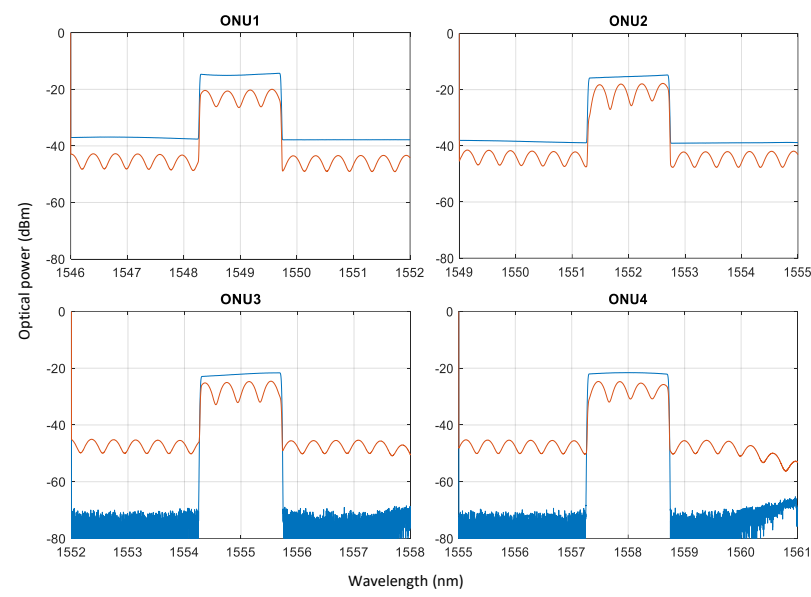

Fig. 4.25: shows the electrical frequency transfer functions measured at each ONU where the TMZIs have been configured with differential delays of 1.76, 3.14, 6.28 and $13.32 \mathrm{ps}$, respectively. The corresponding insets show the recovered power spectral density of the recovered signal and demonstrate effective transmission.

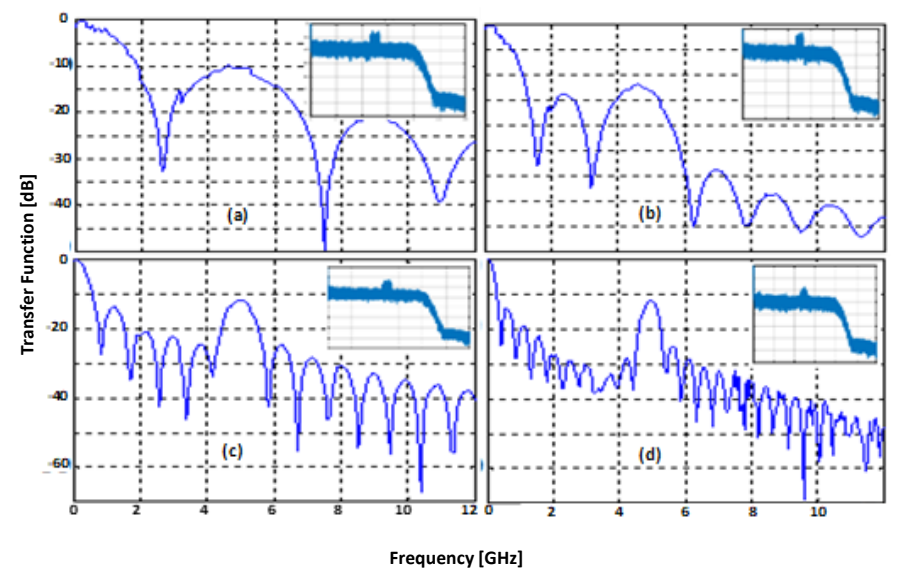

Fig. 4.26: Electrical RF transfer function at: (a) ONU 1, (b) ONU 2, (c) ONU 3 and (d) ONU 4. 
Therefore, any network reconfiguration that involves changes in the fiber link or in the total amount of dispersion will require adjustment of the TMZIs to set the working RF band, whereas links with similar dispersion conditions are transparent to any network reconfiguration.

As the total linewidth of the employed optical source is $85 \mathrm{~nm}$, the network can be scaled to transmit more than 10 optical channels with $6 \mathrm{~nm}$ using the appropriate multiport tunable optical filter.

\subsubsection{Multiple band selection}

In this section, we explore the multiple band selection ability of the proposed system. The proposed scheme for this part is illustrated in Fig. 4.27. Two TMZI in cascade are placed just prior to the downlink detector in order to allow transmission over two different bands, and both variable delay lines are independently adjusted before the optical signal photodetection.

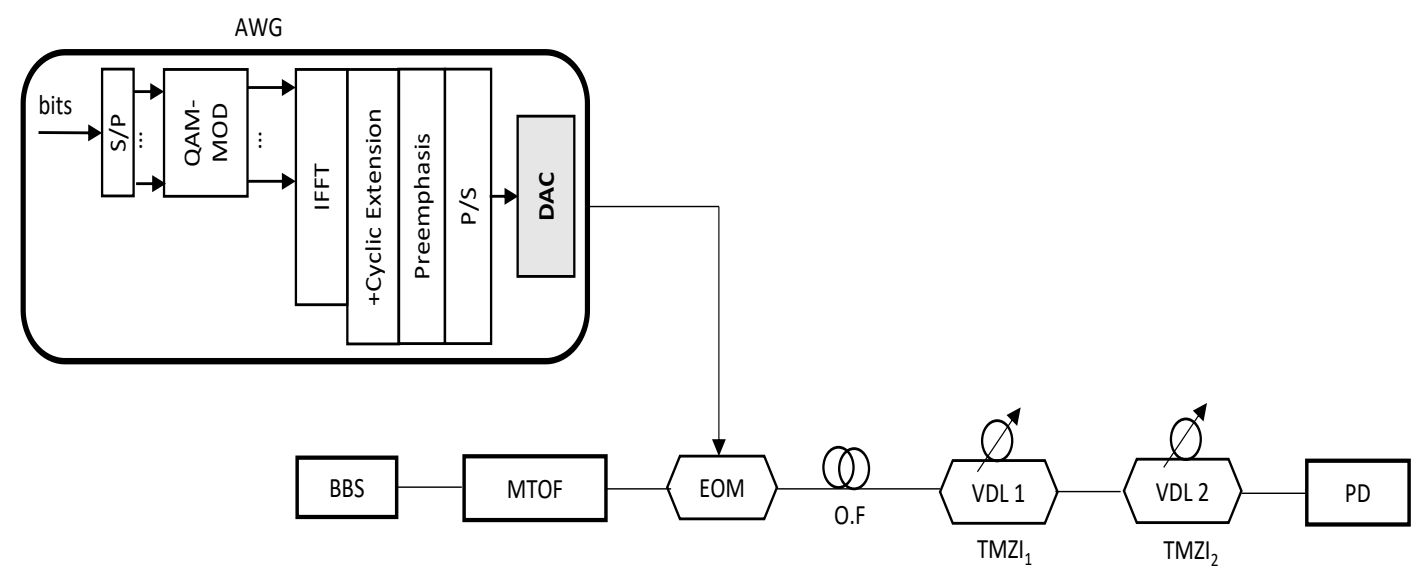

Fig. 4.27: Experimental system with two TMZIs in cascade.

Fig. 4.28 shows the measured RF system transfer function when both of them are set in cascade and the variable delay lines of each one of TMZI are set to obtain transmission bands at 2.5 and $5 \mathrm{GHz}, 3$ and $5 \mathrm{GHz}$ and 3.5 and $6 \mathrm{GHz}$ respectively. 


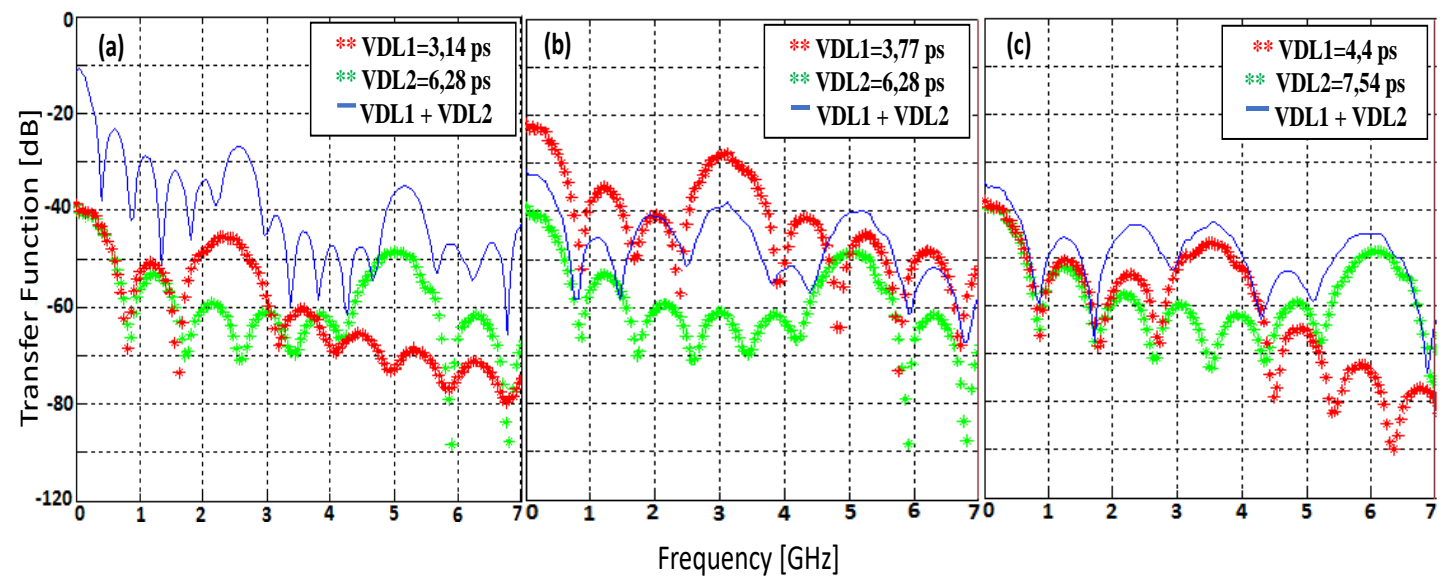

Fig. 4.28: Electrical RF transfer function: (a) TMZIs set at 2.5 and $5 \mathrm{GHz}$, (b) TMZIs set at 3 and $5 \mathrm{GHz}$, (c) TMZIs set at 3.5 and $6 \mathrm{GHz}$.

There are certain intrinsic limitations of the available equipment that have been described throughout this work. In this case, the quality of the received signal was not measured due to limitations in the electrical equipment, which power spectral density of broadband electrical signals was not enough to allow proper signal recovery.

However, better optical received power values are expected and therefore, 7 \% FEC criterion could be satisfied if equipment with higher performance is used. It should be noted that the use of broadband optical sources such as a multi-wavelength transmitter in a WDM access network considerably reduces the cost of implementation, while offering high levels of flexibility and tuning.

\subsection{Conclusion}

In this chapter we have presented a low-cost WDM-OOFDM network based on a centralized broadband optical source at the central office, using dual sideband modulation and reuse of the optical carrier for downlink and uplink transmission below $2 \mathrm{~Gb} / \mathrm{s}$, by introducing an interferometer just before reception. A transmission of the $750 \mathrm{MHz}$ downstream OFDM signal band centred at $5 \mathrm{GHz}$ with a bandwidth of $750 \mathrm{MHz}$ and $0.9 \mathrm{~Gb} / \mathrm{s}$, and actual transmission of the $750 \mathrm{MHz}$ upstream OFDM signal band centred at $3.5 \mathrm{GHz}$, were achieved with an acceptable EVM.

The flexibility of the approach was studied to demonstrate dynamic bandwidth allocation, OFDM band selection in reconfigurable networks and OFDM multi-tuning band selection. Therefore, the proposed network shows moderate bitrates performance but enables bidirectional transmission by using a low-cost solution based on a broadband source, as demonstrated in this chapter. 
Chapter 5

Conclusions and future prospects 


\subsection{Conclusions}

Throughout this thesis, different proposals have been experimentally developed in order to achieve the main objective, which is the implementation and experimental evaluation of an optical transmission system using broadband sources over fiber and free space optical links. Optical carriers in the infrared spectrum have been employed for fiber systems, while visible spectrum is used for free space communications.

Since communication systems based on broadband sources have many advantages such as has been previously demonstrated in [Kim 2007, Gra 2009, Gra 2010, Kan 2014, Dim 2015, Kar 2015, Chi 2017b, Li 2017, Sen 2019, Gao 2019, Yeh 2019, Zho 2019], they undoubtedly offer a great opportunity for the future of optical communication both for free space and optical fiber communications.

However, there is a number of goals to overcome before practical deployment of broadband optical sources in current and future networks. Therefore, the current state and specifications of broadband sources justify the three main contributions of this thesis focusing first on the study of the optical and electronic properties of semiconductors as materials to be employed for manufacturing of LEDs, secondly, on the design of a free space optical transmission (FSO) and lastly, on the proposal of a flexible and bidirectional fiber optic transmission system using broadband optical sources over dispersive fiber links while maintaining low cost and complexity.

\subsubsection{Optoelectronic devices for optical telecommunications}

Optoelectronics is an emerging discipline that uses electronics and optics together. It has important scopes in various fields, particularly in telecommunications. Rapid advances in semiconductor technology hold great promise for optoelectronic devices and their future applications in VLC systems, and they are often used in the manufacturing process of optical components such as LEDs, photovoltaic cells, various optoelectronic devices and other optical communications components.

Semiconductors belonging to the $I I-V I$ family of elements are of great interest to researchers and industrialists because of their characteristics, in particular the energy of the band gap between the visible and ultraviolet light domains, formed by the association of an element from column $I I$ and an element from column VI of the Mendeleev periodic classification.

Among this class of semiconductors, mono zinc chalcogenides are interesting materials for many optoelectronic applications. The interest of these compounds is further enhanced by the possibility of making alloys by combining the elements $I I-V I$, such as the ternary alloys $Z_{n S e_{1-x}} O_{x}$. These components become very attractive due to their special optical properties and their possible applications in light transmission devices, which allows the production of materials with bandgap and optical properties that vary over a very wide range of wavelengths. In ternary $\mathrm{ZnSe}_{1-x} \mathrm{O}_{x}$, new alloys are obtained that allow for the diversification 
of physical properties such as bandgap to meet the important needs of the new generations of devices used for photovoltaic detectors and light-emitting diodes.

The first contribution of this thesis concerns a simulation study using the Wien2k software for the study of the different properties of ternary component $Z n S e_{1-x} O_{x}$, which need to be characterized for the fabrication of optoelectronic devices for optical communications.

The study of the performance of the ternary system $Z n S e_{1-x} O_{x}$ and the relative binary $(Z n S e$ and $\mathrm{ZnO}$ ) for solid-state lighting, the effect of oxygen substitution on the material and their electronic and optical properties (band gap, refractive index, dielectric function, absorption coefficient, the network parameter and the mass modulus) was performed by deploying the Ab-initio method approaches based on the Density Functional Theory (DFT) within the framework of local density approximation (LDA) and generalized gradient approximation (GGA) using the Wien2K method and the FP-LAPW method. The results obtained show that the variation in oxygen concentration leads to several optoelectronic devices with different properties that can be useful as robust solid-state lighting such as LEDs and solar cells.

\subsubsection{Optical wireless communications system}

Visible light communication (VLC) is one of the key areas of wireless communication. It works in the same way as fiber optics, but the transmission medium is free space. The invention of LEDs has led to great advances in the way visible light communication is carried out. LEDs can be used to transmit data, but the transmitter and receiver must be in each other's line-of-sight.

Hence, the second contribution of this thesis is the design of two Visible light communication (VLC) systems using a broadband source such as an LED for signal transmission. The first prototype was designed for analogue transmission of sound, and the second for digital transmission of text and images in simplex mode where Manchester coding was used.

- In the first prototype, an analogic audio signal was transmitted. In reception, we used a photovoltaic cell, which allowed not only the recovery of information but also the wireless transfer of energy/power. This allows us to consider the possibility of an OWC system with new dual functions besides lighting, such as, data transmission and wireless energy/power transmission.

- In the second prototype, transmission of digital data was established for text and images in simplex mode. We have chosen this mode due to a lack of material means. However, we have proposed and implemented an algorithm for the automatic detection of an adaptive decision threshold between Bit 1 and Bit 0 depending on the received light level in order to overcome two problems:

$\circ$ the attenuation of light signal over the distance and

$\circ$ the evolution and sudden change of light intensity in the indoor environment.

Consequently, based on this algorithm and Manchester Code, we were able to perform text and image transmissions with zero detected errors. The reason for this choice is related to the fact that this code is indicated by the IEEE 802.15.7 standard for these applications. The 
experimental results obtained have confirmed acceptable performance in terms of received signal quality and detected errors.

However, both prototypes need to be improved in terms of equipment's, mode of transmission (extension to duplex mode), increasing data rate and evaluation of different modulation techniques.

\subsubsection{Bidirectional WDM-OOFDM access network based broadband source}

The third contribution is devoted to the implementation of a bidirectional WDM-OOFDM optical communication system using a broadband source.

The implementation of a system capable of transmitting and receiving signals in the OOFDM modulation format was done at the Photonics Research Labs (PRL) of the Polytechnic University of Valencia, Spain. A complex experimental setup was required and costly equipment was employed for this work. Concerning the generation and reception of the OFDM signal we opted for the implementation of a Matlab-based signal processor with a series of configurable parameters that were described in Chapter 4.

A BBS was employed to transmit OFDM signals in optical networks by using a MZM interferometer as a dispersion mitigation technique which allowed to transmit signals within a given frequency band. The innovative contribution of this Thesis was to develop a full duplex transmission scenario in a centralized network with the main objective of minimizing the network costs in the $\mathrm{CO}$ transceiver rather than increasing the capacity.

Therefore, a low-cost WDM-OOFDM network based on a CO centralized broadband optical source using dual sideband modulation and optical carrier reuse for downlink and uplink transmission below $2 \mathrm{~Gb} / \mathrm{s}$ has been experimentally proven. We have also explored the great potential offered by the BBS to implement a reconfigurable two-way network with dynamic bandwidth allocation, multi-band operation and multiple users. However, further work is proposed to improve the quality of the electrical signal generated to allow the transmission of the signal at higher bit rates.

\subsection{Future research lines}

The main contribution of this Thesis leads to several experimental achievements with regards to the materials and applications related to optical broadband sources both in the visible and in the infrared spectral ranges. However, besides the inherent difficulties related to laboratory budget and available equipment, the contributions of this thesis lead to several issues to be addressed as directions for future research.

In this thesis, a series of deficiencies related to the quality of the signal as a function of distance for VLC systems were highlighted, practically due to the characteristics of the equipment used. One of the most important issues that should be improved is the communication range. In this case, the problem is that the power of the signal and consequently the SNR drops significantly when the communication range increases. Increasing the receiver gain is a suitable solution but still, it has its limits which imply that additional measures should be taken. 
Moreover, VLC has been proven to be capable of very high data rates of up to $3 \mathrm{~Gb} / \mathrm{s}$ or more in the literature. These high data rates are achieved through more complex modulations such as OFDM or multi-level codes and more expensive equipment is required. Future research should study OFDM, PAM and also advanced formats such as filter bank multicarrier (FBMC) modulation techniques with increased spectral efficiency.

In the OFDM transmission system over optical links, the limitations were mainly due to the voltage at the output of the AWG equipment, which is limited to $1 \mathrm{VPP}$, resulting in the limitation of the electrical signal power. A possible solution to this problem is the introduction of a variable radio-frequency amplifier at the output of the AWG, with an analogue filter provided maximum power in the modulator is not exceeded. Improving the quality of the electrical signal leads to higher quality signals and higher bitrates would be achieved in our system.

Other optical broadband sources can be explored under the approach considered in this Thesis with the aim of demonstrating effective multiband full-duplex transmission with high quality recovered signals, and therefore, increase the potentiality of the proposed low cost centralized networks.

The combination of a transmission system consisting of an optical fiber and a free space link (VLC) is envisaged. With an OFDM signal whose data are modulated in $16-Q A M$ or with the use of other modulation techniques such as PAM, this is an interesting starting point for the implementation of hybrid FSO/fiber optic systems which allow higher transmission speeds to be achieved for various applications as demonstrated in the preliminary experiments. Such hybrid links will be very relevant in future networks deployment as part of the mobile backhaul in access communication networks.

Under the use of broadband sources for saving costs and energy efficiency reasons among others, all these improvements will further increase the performance of visible light communication systems over free space and infrared communication systems over fiber and free space optics towards future deployment of flexible and high performance networks as required by current and future services. However, for VLC still needs the support of the solid state lighting and the photodiode industry. Future faster switching LEDs will enable higher data rates in indoor applications, whereas more sensitive photoelements will enable longer communication distances. 
Bibliography 
[Agr 1995] G. P. Agrawal, Nonlinear Fiber Optics, 2nd ed. Academic, ISBN: 9781483288031 (1995).

[And 1975] O.K. Andersen, "Linear methods in band theory", Phys. Rev. B, Vol.12, no. 8, pp. 3060-3083 (1975).

[Aou 2009] S. Aouadi, "Calcul ab initio des propriètés structurales, électroniques, élastiques et dynamiques des semiconducteurs III-V à base de phosphore", doctoral thesis, Badji Mokhtar Annaba University Algeria (2009).

[Arm 2009] J. Armstrong, "OFDM for optical communications," Journal of Lightwave Technology, Vol. 27., no. 3, pp. 189-204 (2009).

\section{B}

[Baa 2006] H. Baaziz, Z. Charifi, F. El Haj Hassan, S. J. Hashemifar and H. Akbarzadeh, "FP-LAPW investigations of Zn1-xBexS, Zn1-xBexSe and Zn1-xBexTe ternary alloys", Physica Status Solidi (b), Vol. 243, no. 6, pp. 1296-1305 (2006).

[Bac 2019] Y. A. Bachtiar and T. Adiono, "PAM-4 Modulator-Demodulator Design Modeling for Visible Light Communication (VLC)", 2019 International Symposium on Electronics and Smart Devices (ISESD), Badung-Bali, Indonesia, pp. 1-5 (2019).

[Ben 2018] J. Benítez and J. Mora, "Third-Order Dispersion Compensation for Resolution Enhancement in RF Interferometry", 2018 20th International Conference on Transparent Optical Networks (ICTON), pp. 1-4 (2018).

[Ben 2014] M. BENHAMIDA, "Propriétés structurale, élastiques et électronique d'alliages de nitrure des métaux de transitions", doctoral thesis, Ferhat Abbas Setif 1 University Algeria (2014).

[Bla 2020] P. Blaha, K. Schwarz, F. Tran, R. Laskowski, G.K.H. Madsen and L.D. Marks, "WIEN2K: An APW+lo program for calculating the properties of solids", Journal of Chemical Physics, Vol. 152, no.7 (2020).

[Bla 2008] P. Blaha, K. Schwarz, G.K.H. Madsen, D. Kvasnicka and J. Luitz, "WIEN2k: An Augmented Plane Wave Plus Local Orbitals Program for calculating Crystal Properties", Vienna University of Technology, Vienna, Austria (2008).

[Bla 1990] P. Blaha, K. Schwarz, P. Sorantin and S. B. Trickey, "Full-potential, linearized augmented plane wave programs for crystalline systems", Computer Physics Communications, Vol. 59, no. 2, pp. 399- 415 (1990).

[Bou 2016] A.S. Bouarissa, H. Choutri, M.A. Gheboulia and N. Bouarissa, "Ab initio study of electronic structure and lattice properties of $\mathrm{ZnSe}_{1-\mathrm{x}} \mathrm{O}_{\mathrm{x}}$ ", Optik, 
Vol. 127, no.4, pp. 1889-1892 (2016).

[Bor 1927] D.Born and J.R. Oppenheimer, "Quantum Mechanics of molecular systems”, Annalen der Physik, Vol. 84, pp.457-484 (1927).

[Buh 2014] A. M. Buhafa, B. M. Al-Nedawe, M. J. N. Sibley and P. J. Mather, "VLC system performance using Dicode Pulse Position Modulation over an indoor diffuse link," $201422^{\text {nd }}$ Telecommunications Forum Telfor (TELFOR), Belgrade, pp. 605-608 (2014).

\section{C}

[Cap 1999] J. Capmany, F. J. Fraile-Peláez and J. Martí, "Dispositivos de Comunicaciones Ópticas”, Síntesis editorial, ISBN :84-7738-634-X (1999).

[Cap 1998] J. Capmany, F. J. Fraile-Peláez and J. Martí, "Fundamentos de Comunicaciones Ópticas", Síntesis editorial, ISBN 10: 8477385998 (1998).

[Car 2003] J. B. Carruthers, "Wireless infrared communications," Wiley Encyclopedia of Telecommunications (2003).

[Car 1994] J. B. Carruthers and J. M. Kahn, "Multiple-subcarrier modulation for nondirected wireless infrared communication," 1994 IEEE GLOBECOM. Communications: The Global Bridge, San Francisco, CA, USA, Vol.2, pp. 1055-1059 (1994).

[Cep 1980] D. M. Ceperley and B. J. Adler, "Ground State of the Electron Gas by a Stochastic Method”, Physical Review Letters, Vol.45, no.7, pp.566-569 (1980).

[Che 2019] D. Che, S. Chandrasekhar, X. Chen, G. Raybon, P. Winzer, C.Sun and W.Shieh, "Single-Channel Direct Detection Reception beyond $1 \mathrm{~Tb} / \mathrm{s}$ ", 2019 Optical Fiber Communications Conference and Exhibition (OFC), San Diego, CA, USA, pp. 1-3 (2019).

[Che 2014] G. F. R. Chen, T. Wang, C. Donnelly and D. T. H. Tan, "Generation of second and third order optical dispersion using nonlinearly chirped silicon waveguide gratings", in CLEO: 2014, OSA Technical Digest (online) (Optical Society of America, 2014), paper AW1H.1 (2014).

[Che 2013a] C.C. Cheng, W.Y. Chen, H.H. Ke, C.Y. Chen, J.I. Chyi and T.M. Hsu, "Composition dependent raman scattering of $\mathrm{ZnSe}_{1-x} \mathrm{O}_{x}$ ", Solid State Communications, Vol.172, pp.20-23 (2013).

[Che 2013b] C. Y. Chen, C. Y. Yang, J. I. Chyi and C. H. Wu, "Optical and electrical properties of $\mathrm{ZnSeO}$ alloys grown by plasma-assisted molecular beam epitaxy", Elsevier, Journal of Crystal Growth, Vol. 378, pp 180-183 (2013). 
[Chi 2018] F.I Chicharro, "Técnicas ópticas de transmisión avanzadas para redes OOFDM-WDM", doctoral thesis of the Polytechnic University of Valencia, Spain (2018).

[Chi 2017a] F.I. Chicharro, B. Ortega, M. De Diego and J. Mora, "OOFDM Signal Transmission Using a Single Optical Broadband Source", in IEEE Photonics Technology Letters, Vol. 29, no. 7, pp. 563-566 (2017).

[Chi 2017b] F.I. Chicharro, B. Ortega, B. De Diego and J. Mora, "Broadband optical sources for low-cost WDM-MB-OFDM networks", in IEEE Communications Letters, Vol. 21, no. 8, pp. 1759-1762 (2017).

[Chi 2015] Y.-C. Chi, D.-H. Hsieh, C.-T. Tsai, H.-Y. Chen, H.-C. Kuo, and G-R. Lin, "450-nm GaN laser diode enables high-speed visible light communication with 9-Gbps QAM OFDM," Optics Express, Vol. 23, no. 10, pp. 1305113059 (2015).

[Chi 2014] N. Chi, Y. Wang, Y. Wang, H. Xing and X. Lu, "Ultra-high-speed single red-green-blue light-emitting diode-based visible light communication system utilizing advanced modulation formats," Chinese Optics Letters, Vol. 12, no. 1, pp. 010605 (2014).

[Cho 2018] M. Z. Chowdhury, M.T. Hossan, A. Islam,Y and M. Jang, "A Comparative Survey of Optical Wireless Technologies: Architectures and Applications", in IEEE Access, Vol. 6, pp. 9819-9840 (2018).

[Cho 2013] C. W. Chow, C. H. Yeh, Y. F. Liu and P. Y. Huang, "Mitigation of Optical Background Noise in Light-Emitting Diode (LED) Optical Wireless Communication Systems", IEEE Photonics Journal, Vol.5, no. 1, pp. 7900307-7900307 (2013).

[Cho 2010] Y. S. Cho, J. Kim, W. Y. Yang, Chung and G. Kang, "MIMO-OFDM WIRELESS COMMUNICATIONS WITH MATLAB", in IEEE Press, ISBN: 978-0-470-82561-7 (2010).

[Cis 2018] Cisco. Visual Networking Index (VNI) Complete Forecast Update, 20172022, Tech.rep.Cisco (2018).

[Cvi 2012] N. Cvijetic, M. Cvijetic, M.F. Huang, E. Ip, Y.K. Huang and T. Wang, "Terabit optical access networks based on WDM-OFDMA-PON", Journal of Lightwave Technology, Vol. 30, pp. 493-503 (2012).

\section{D}

[Dar 2017] M. C. Darji, "Audio Signal Processing: A Review of Audio Signal Classification Features", International Journal of Scientific Research in Computer Science, Engineering and Information Technology, Vol.2, no. 3 (2017).

[Dim 2015] S. Dimitrov and H. Haas, "Principles of LED Light Communications: Towards Networked Li-Fi", Cambridge, U.K.: Cambridge University 
Press (2015).

[Dir 1930] P. A. M. Dirac, Mathematical Proceedings of the Cambridge Philosophical Society, Vol. 26, no. 3, pp. 376-385 (1930).

[Dur 1997] M. Durkin, M. Ibsen, M. J. Cole and R. I. Laming, "1 m long continuously-written fiber Bragg gratings for combined second-and thirdorder dispersion compensation," Electronics Letters, Vol.33, no.22, pp.1891-1893 (1997).

\section{$\mathbf{E}$}

[Eis 2002] H.J. Eisler, V.C. Sundar, M.G. Bawendi, M. Walsh, H.I. Smith and V. Klimov, "Color-selective semiconductor nanocrystal laser," Applied Physics Letters, Vol. 80, no. 24, pp. 4614-4616 (2002).

[Elg 2011] H. Elgala, R. Mesleh and H. Haas, "Indoor optical wireless communication: potential and state-of-the-art," in IEEE Communications Magazine, Vol. 49, no. 9, pp. 56-62 (2011).

[Erg 2015] O. Ergul, E. Dinc and O. B. Akan, "Communicate to illuminate: State-ofthe-art and research challenges for visible light communications", Physical Communication, Vol. 17, pp 72-85 (2015).

[Eso 2019] E. Eso, A. Burton, N. B. Hassan, M. M. Abadi, Z. Ghassemlooy and S. Zvanovec, "Experimental Investigation of the Effects of Fog on Optical Camera-based VLC for a Vehicular Environment", IEEE, 15th International Conference on Telecommunications (ConTEL), Graz, Austria, pp. 1-5 (2019).

\section{$\mathbf{F}$}

[Fal 2016] M. Fall, G. Yan and Z. Zhong, "An integrated Single mode fiber communication and visible light communication system based on OFDM with Hadamard Transform", IEEE, 15th International Conference on Optical Communications and Networks (ICOCN), Hangzhou, pp. 1-3 (2016).

[Fan 2018] J. Fang, M. Bi, S. Xiao, G. Yang, L. Liu, Y. Zhang and W. Hu, "PolarCoded MIMO FSO Communication System Over Gamma-Gamma Turbulence Channel With Spatially Correlated Fading”, IEEE/OSA Journal of Optical Communications and Networking, Vol. 10, no. 11 (2018).

[Far 2019] Z. A. Farhat, M. H. Ahfayd, P. J. Mather and M. J. N. Sibley, "Improved BER for offset pulse position modulation using priority decoding over VLC system", 2019 Wireless Days (WD), Manchester, United Kingdom, 
pp. 1-4 (2019).

[Fer 1927] E. Fermi, "Un metodo static per la determinazione di alcune priorietà dell'atome", Rendiconti Accademia dei Lincei, Vol. 6, pp. 602-607 (1927).

\section{G}

[Gao 2019] M. Gao, C. Li and Z. Xu, "Performance enhancement of LED-based indoor OFDM-VLC system using digital chaotic scheme", Optics Communications, Vol. 439, pp.21-26 (2019).

[Gao 2017] Y. Gao, S. Li, X. Xue, X. Zheng, H. Zhang and B. Zhou, "Precise Measurement of Fiber Third-Order Dispersion Using Transfer Function of a Microwave Photonic Filter", Journal of Lightwave Technology, Vol. 35, no. 22 , pp. 4865-4870 (2017).

[Gao 2011] Y. Gao, J. Yu, J. Xiao, Z. Cao, F. Li and L. Chen, "Direct-detection optical OFDM transmission system with pre-emphasis technique", Journal of Lightwave Technology, Vol. 29, no. 14, pp. 2138-2145 (2011).

[Gér 2013] L. Gérard, "Structures de semiconducteurs II-VI à alignement de bandes de type II pour le photovoltaïque", doctoral thesis of GRENOBLE University France (2013).

[Gfe 1979] F.R. Gfeller and U. H. Bapst, "Wireless in-house data communication via diffuse infrared radiation," Proceedings of the IEEE, Vol. 67, no. 11, pp. 1474-1486 (1979).

[Gha 2017] Z. Ghassemlooy, L. N. Alves, S. Zvánovec and M.A. Khalighi, "Visible Light Communications Theory and Applications", CRC Press, International Standard Book Number-13: 978-1-4987-6753-8 (2017).

[Gha 2016] Z. Ghassemlooy, P. Luo and S. Zvanovec, "Optical Camera Communications", pp. 547-568, Springer (2016).

[Gha 2013] Z. Ghassemlooy, W. Popoola and S. Rajbhandari, "Optical Wireless Communications System and Channel Modelling with MATLAB”, CRC Press, ISBN: 978-1-4398-5188-3 (2013).

[Gid 2010] R.P. Giddings, E. Hugues-Salas, X.Q. Jin, J.L. Wei and J. M. Tang, "Experimental Demonstration of Colourless Real-Time End-to-End Optical OFDM Transmission at $7.5 \mathrm{~Gb} / \mathrm{s}$ over $25 \mathrm{~km}$ SSMF Using a $1 \mathrm{GHz}$ RSOA”, IEEE Future Network \& Mobile Summit, Florence, Italy, pp. 1-6 
(2010).

[God 2016] R. S. J. Godwin, K. Veena and D. S. Kumar, "Performance analysis of direct detection Flip-OFDM for VLC system", 2016 International Conference on Emerging Trends in Engineering, Technology and Science (ICETETS), Pudukkottai, India, pp. 1-5 (2016).

[Gra 2013] F. Grassi, "Centralized transmission techniques for full-duplex reconfigurable WDM optical access networks", doctoral thesis of the Polytechnic University of Valencia, Spain (2013).

[Gra 2010] F. Grassi, J. Mora, B. Ortega and J. Capmany, "Radio over fiber transceiver employing phase modulation of an optical broadband source", OSA Optics Express, Vol. 18, no. 21, pp. 21750-21756 (2010).

[Gra 2009] F. Grassi, J. Mora, B. Ortega and J. Capmany, "Subcarriers multiplexing tolerant dispersion transmission system employing optical broadband sources", Optics Express, Vol. 17, no.6, pp. 4740-4751 (2009).

\section{$\mathbf{H}$}

[Haa 2011] H. Haas, "Wireless data from every light bulb", TED Website [Online]. 2011 Available: http://bit.ly/tedvlc.

[Ham 1979] D. R. Hamann, "Semiconductor Charge Densities with Hard-Core and Soft-Core Pseudopotentials", Physical Review Letters, Vol. 42, no. 10, pp. 662 (1979).

[Han 2012] L. Hanzoetal, "Wireless myths, realities, and futures: from 3G/ 4G to optical and quantum wireless", in Proceedings of the IEEE, Vol. 100, no. Special Centennial Issue, pp. 1853-1888 (2012).

[Han 2008] F. Hanson, S. Radic, "High bandwidth underwater optical communication,” Applied Optics., Vol. 47, no. 2, pp. 277-283 (2008).

[Han 2000] L. Hanzo, W. Webb, and T. Keller, "Single- and Multi-Carrier Quadrature Amplitude Modulation", Wiley, Chichester, 2nd edition, ISBN-13 : 9780471492399 (2000).

[Hao 2009] X. Hao, K. Qiu, C. Zhang and Y. Li, "On the Timing Synchronization Methods for Optical Orthogonal Frequency Division Multiplexing (OOFDM) Systems: Comparisons and Improvement", IEEE Asia Communications and Photonics conference and Exhibition (ACP), Shanghai, China, pp. 1-11 (2009).

[Har 1974] W. A. Harrison, "Effective charges and piezoelectricity",Physical Review B, Vol. 10, pp. 767 (1974). 
[Har 1974b] W. A. Harrison and S.Ciraci, "Bond-orbital model. II", Physical Review B, Vol.10, pp.1516 (1974).

[Har 1928] D. R. Hartree, "The Wave Mechanics of an Atom with a non-Coulomb Central Field. Part III. Term Values and Intensities in Series in Optical Spectra", Mathematical Proceedings of the Cambridge Philosophical Society, Vol. 24, no.3, pp. $426-437$ (1928).

[Hay 1988] S. Haykin, "Digital Communications", John Wiley \& Sons, New York, NY, ISBN:978-0-471-62947-4 (1988).

[Her 2002] C. I. Hernández, "Optical properties and electronic structure of wide band gap II-VI semiconductors", in II-VI Semiconductor Materials and their Applications, Taylor et Francis New York, pp.113-170, ISBN: 9780203751305 (2002).

[Hoh 1964] P. Hohenberg and W. Kohn, "Inhomogeneous Electron Gas", Physical Review B, Vol. 136, no. 864 (1964).

[Hos 2016] M.S. Hossain and T. Shimamura, "Low-Complexity Null SubcarrierAssisted OFDM PAPR Reduction with Improved BER", in IEEE Communications Letters, Vol. 20, no. 11, pp. 2249-2252 (2016).

[Hou 2006] J. Hou and D. C. O'Brien, "Vertical handover-decision-making algorithm using fuzzy logic for the integrated Radio-and-OW system", in IEEE Transactions on Wireless Communications, Vol. 5, no.1, pp. 176-185 (2006).

[Hra 2013] S. Hranilovic, L. Lampe and S. Hosur, "Visible light communications: the road to standardization and commercialization", in IEEE Communications Magazine, Vol. 51, Issue: 12, ISSN: 0163-6804, pp. 24-54 (2013).

[Hua 2015] X. Huang, S. Chen, Z. Wang, J. Shi, Y. Wang, J. Xiao and N. Chi, "2.0$\mathrm{Gb} / \mathrm{s}$ visible light link based on adaptive bit allocation OFDM of a single phosphorescent white LED”, in IEEE Photonics Journal, Vol. 7, no. 5, pp. $1-8(2015)$.

\section{I}

[IES 2000] IESNA Lighting Handbook, 9th Edition, (New York; IESNA, 2000), 274.

[Isl 2018] M. S. Islim, S. Videv, M. Safari, E. Xie , J. J. D. McKendry, E. Gu and M. D. Dawson, "The Impact of Solar Irradiance on Visible Light Communications", Journal of Lightwave Technology, Vol. 36, no. 12, pp. 2376-2386 (2018). 
[Jan 2007] S. L. Jansen, I. Morita, N. Takeda and H. Tanaka, "20-Gb/s OFDM transmission over 4160-km SSMF enabled by RF-pilot tone phase noise compensation", in Optical Fiber Communication Conference and Exposition and The National Fiber Optic Engineers Conference, Anaheim, CA, 2007, Paper PDP 15 (2007).

[Jun 1998] D. K. Jung, S. K. Shin, C.-H. Lee and Y. C. Chung, "WavelengthDivision-Multiplexed Passive Optical Network Based on SpectrumSlicing Techniques", in IEEE Photonics Technology Letters, Vol. 10, no.9, pp. 1334-1336 (1998).

\section{$\mathbf{K}$}

[Kah 1997] J.M. Kahn and J.R. Barry, "Wireless infrared communications", in Proceeding of the IEEE, Vol. 85, no. 2, pp. 265-298 (1997).

[Kan 2014] B.W. Kang, K. Lee, S.B. Lee and C.H. Kim, "Colorless amplified WDMPON employing broadband light source seeded optical sources and channel-by-channel dispersion compensators for $>100 \mathrm{~km}$ Reach," Journal of the Optical Society of Korea, Vol. 18, no. 5, pp. 436-441 (2014).

[Kar 2015] D. Karunatilaka, F. Zafar, V. Kalavally and R. Parthiban, "LED Based Indoor Visible Light Communications: State of the Art", in IEEE Communication Surveys \& Tutorials, Vol. 17, no. 3, 3th Quarter (2015).

[Kas 2018] M. Kashef, M. Abdallah and N. Al-Dhahir, "Transmit Power Optimization for a Hybrid PLC/VLC/RF Communication System", in IEEE Transactions On Green Communications And Networking, Vol. 2, no. 1, pp. 234-245 (2018).

[Kha 2017a] M-A Khalighi, S. Long, S. Bourennane and Z. Ghassemlooy, "PAM- and CAP-Based Transmission Schemes for Visible-Light Communications", in IEEE Access, Vol. 5, pp. 27002-27013 (2017).

[Kha 2017b] L. U. Khan, "Visible light communication: Applications, architecture, standardization and research challenges", in Digital Communications and Networks, Vol. 3, no. 2, pp. 78-88 (2016).

[Kha 2014] M. A. Khalighi and M. Uysal, "Survey on free space optical communication: A communication theory perspective", in IEEE Communications Surveys \& Tutorials, vol. 16, no. 4, pp. 2231-2258, 4th Quarter (2014).

[Kil 2002] D. Killinger, "Free space optics for laser communication through the air", in Optics and Photonics News, Vol. 13, no.10, pp. 36-42 (2002).

[Kim 2007] J. Cho, J. Kim, D. Gutierrez and L. G. Kazovsky, "Broadcast Transmission in WDM-PON using a Broadband Light Source", 
OFC/NFOEC 2007 - 2007 Conference on Optical Fiber Communication and the National Fiber Optic Engineers Conference, Anaheim, CA, USA, pp. 1-3 (2007).

[Kli 2017] A. Kliks and P. Kryszkiewicz, "Multichannel simultaneous uplink and downlink transmission scheme for flexible duplexing”, EURASIP Journal on Wireless Communications and Networking, no. 111 (2017).

[Koc 2000] W. Koch and M. C. Holthausen, "A Chemist's Guide to Density Functional Theory", Wiley-VCH, Weinheim, Germany, ISBN:9783527303724 (2000).

[Koh 1995] B. kohler, P. Ruggerone, S. Wilke and M. Scheffler, "Frustrated Hinduced instability of Mo(110)", Physical Review letters, Vol. 74, no. 1387 (1995).

[Koh 1965] W. Kohn and L. Sham, "Self-Consistent Equations Including Exchange and Correlation Effects", Physical Review A, Vol. 140, no.11331(965).

[Kom 2004] T. Komine and M. Nakagawa, "Fundamental analysis for visible-light communication system using LED lights", in IEEE Transactions on Consumer Electronics, Vol. 50, no. 1, pp. 100-107 (2004).

[Kum 2019] A. Kumar and V Sudha, "Optical Power Distribution and Statistical Analysis of Indoor Visible Light Communication", 2019 TEQIP III Sponsored International Conference on Microwave Integrated Circuits, Photonics and Wireless Networks (IMICPW), Tiruchirappalli, India, pp. 383-386 (2019).

\section{$\mathbf{L}$}

[Lac 2002] J.P.R. Lacey, G.J. Pendock and R.S. Tucker, “All-optical 1300-nm to 1550-nm wavelength conversion using cross-phase modulation in a semiconductor optical amplifier", in IEEE Photonics Technology Letters, Vol. 8, no. 7, pp. 885-887 (2002).

[Lee 2011] K. Lee, H. Park, "Modulations for Visible Light Communications With Dimming Control”, in IEEE Photonics Technology Letters, Vol. 23, no. 16, pp. 1136-1138 (2011).

[Lee 2007] Y. J. Lee, J. Bae, K. Lee, J-M. Jeong and S. B. Lee, “Tunable Dispersion and Dispersion Slope Compensator Using Strain-Chirped Fiber Bragg Grating”, in IEEE Photonics Technology Letters , Vol. 19, no. 10, pp. $762-764$ (2007).

[Li 2019a] X. Li, C. Min, S. Gao, Y. Wang, X. Chen and H. Chen, "Experimental demonstration of a real-time multi-channel uplink VLC system", Optics Communications, Vol. 453, pp. 124420 (2019). 
[Li 2019b] R. Li, T. Chen, L. Fan and A. Dang, "Performance Analysis of a Multiuser Dual-Hop Amplify-and-Forward Relay System With FSO/RF Links", in IEEE/OSA Journal of Optical Communications and Networking, Vol. 11, no. 7, pp. 362-370 (2019).

[Li 2016] S. Li, A. Pandharipande and F. M. J. Willems, "Unidirectional Visible Light Communication and Illumination with LEDs", in IEEE Sensors Journal, Vol. 16, no. 23, pp. 8617-8626 (2016).

[Lin 2010] Y.C. Lin, H.L. Chung, W.C. Chou, W.K. Chen, W.H. Chang, C.Y. Chen and J.I. Chyi, "Carrier dynamics in isoelectronic ZnSe 1-xOx semiconductors”, Applied Physics Letters, Vol. 97, pp. 041909 (2010).

[Lin 2012] B. Lin, J. Li, H. Yang, Y. Wan, Y. He and Z. Chen, "Comparison of DSB and SSB transmission for OFDM-PON [invited]," Journal of Optical Communications and Networking, Vol. 4, no. 11, pp. B94-B100 (2012).

[Lou 2014] S. Louvros, D. Fuschelberger, N. Sklavosm M. Hübner, D. Goehringer and P. Kitsos, "VLC Technology for LTE Indoor Planning", SystemLevel Design Methodologies for Telecommunication, Springer, ISBN: 978-3-319-00662-8, pp. 23 (2014).

[Lor 2020] N. Lorrière, N. Bétrancourt, M. Pasquinelli, G. Chabriel, J. Barrère, L. Escoubas, J-L Wu, V. Bermudez, C. M. Ruiz and J-J Simon, "Photovoltaic Solar Cells for Outdoor LiFi Communications", in IEEE, Journal Of Lightwave Technology, Vol. 38, no. 15, pp. pp. 3822-3831 (2020).

[Lu 2016] I. Lu, C. Yeh, D. Hsu and C. Chow, "Utilization of 1-GHz VCSEL for 11.1-Gbps OFDM VLC Wireless Communication," in IEEE Photonics Journal, Vol. 8, no. 3, pp. 1-6 (2016).

[Lu 2015] H-H. Lu, C-Y. Li, C-A. Chu, T-. Lu, B-R. Chen, C-J. Wu and Dai-Hua Lin, "10 m/25 Gbps LiFi transmission system based on a two-stage injection-locked $680 \mathrm{~nm}$ VCSEL transmitter," Optic Letters, Vol. 40, no. 19, pp. 4563-4566 (2015).

[Luo 2017] J. Luo, L. Fan and H. Li, "Indoor Positioning Systems Based on Visible Light Communication: State of the Art", in IEEE Communications Surveys \& Tutorials, Vol. 19, no. 4, pp. 2871-2893, Fourth quarter (2017).

\section{M}

[Mal 2015] A. Malik and P. Singh, "Free space optics: Current applications and future challenges", International Journal of Optics, Vol. 2015, Art. no. 945483 (2015).

[Mat 2019] L. E. M. Matheus, A. B. Vieira, L. F. M. Vieira, M. A. M. Vieira and O. 
Gnawali, "Visible Light Communication: Concepts, Applications and Challenges", in IEEE Communications Surveys \& Tutorials, Vol. 21 , no. 4 , pp. 3204-3237, Fourth quarter (2019).

[Mat 2014] Mathworks, "Design figital filters", mathworks.com/help/signal/ref/designfilt.html, Matlab. 2014.

[Min 2020] C. Min, X. Chen, X. Mao, X. Li, T. Pan, Q. Sun and H. Chen, "A Novel Method for Constructing VLC Equalizer With Active-Passive Hybrid Network", in IEEE Photonics Journal , Vol. 12 , no. 2 , pp. 1-10, Art no. 7500310 (2020).

[Mmb 2016] P. F. Mmbaga,, J. Thompson and H. Haas, "Performance Analysis of Indoor Diffuse VLC MIMO Channels Using Angular Diversity Detectors", in IEEE Journal of Lightwave Technology, Vol. 34 , no. 4, pp. 1254-1266 (2016).

[Mob 2012] E. Mobil, Report: "2012 The Outlook for Energy: A view to 2040", available at: exxonmobil.com/energyoutlook (2012).

\section{$\mathbf{N}$}

[Nab 2003] Y. Nabetani, T. Mukawa, T. Okuno, Y. Ito, T. Kato and T. Matsumoto, "Structure and optical properties of $\mathrm{ZnSeO}$ alloys with $\mathrm{O}$ composition up to 6.4\%", Elsevier, Materials Science in Semiconductor Processing, Vol. 6, no. 5-6, pp. 343-346 (2003).

[Ndj 2018] A. R. Ndjiongue, T. M. N. Ngatched and H. C. Ferreira, "On the Indoor VLC Link Evaluation Based on the Rician K-Factor", in IEEE Communications Letters, Vol. 22, no. 11, pp. 2254-2257 (2018).

[Nak 2007] M. Nakagawa, "Visible Light Communications", Conference on Lasers and Electro-Optics/Quantum Electronics and Laser Science Conference and Photonic Applications Systems Technologies, OSA Technical Digest Series (CD) (Optical Society of America, 2007), paper CTuI5 (2007).

[Nlo 2018] S. M. Nlom, A. R. Ndjiongue and K. Ouahada, "Cascaded PLC-VLC Channel: An Indoor Measurements Campaign”, IEEE Access, Vol. 6, 2018, pp. $25230-25239$ (2018).

[Nos 2012] M. Noshad and M. Brandt-Pearce, "Multilevel pulse-position modulation based on balanced incomplete block designs", in: Proceedings of the Global Communications Conference (GLOBECOM), Anaheim, CA, USA, pp. 2930-2935 (2012).

\section{O}

[Oue 1995] F. Ouellette, P. A. Krug, T. Stephens, G. Dhosi and B. Eggleton, 
"Broadband and WDM dispersion compensation using chirped sampled fibre Bragg gratings", Electronics Letters, Vol. 31, no.11, pp. 899-901 (1995).

\section{$\mathbf{P}$}

[Pam 2018] P. D. Pamungkasari, I. Shubhi, F. H. Juwono, P. D. Mariyam and D. Gunawan, "Time Domain Cyclic Selective Mapping for PAPR Reduction in MIMO-OFDM Systems", in IEEE International Conference on Innovative Research and Development (ICIRD), Bangkok, Thailand, pp. 1-4 (2018).

[Par 2007] S.-B. Park, D. K. Jung, H.S. Shin, D.J. Shin, Y.-J. Hyun, K. Lee and Y.J. Oh, "Information broadcasting system based on visible light signboard", Presented at Wireless and Optical Communications 2007, Montreal, Canada (2007).

[Pat 2015] P.H. Pathak, X. Feng, P. Hu and P. Mohapatra, "Visible light communication, networking, and sensing: a survey, potential and challenges", in Communications Surveys \& Tutorials, Vol. 17, no. 4, pp. 2047-2077, Fourth quarter (2015).

[Pel 1980] A. Peled and A. Ruiz. "Frequency domain data transmission using reduced computational complexity algorithms", in International Conference on Acoustics, Speech, and Signal Processing. Vol. 5, pp. 964967 (1980).

[Pen 1962] D. R. Penn, "Wave-Number-Dependent Dielectric Function of Semiconductors”, in Physical Review, Vol. 128, no. 5, pp. 2093 (1962).

[Per 1992] J. P. Perdew, J. A. Chevary, S. H. Vosko, K. A. Jackson, M. R. Peederson, D. J. Singh and C. Fiolhais, "Atoms, molecules, solids, and surfaces: Applications of the generalized gradient approximation for exchange and correlation”, Physical Review B, Vol. 46, no.11, pp. 6671 (1992).

[Per 1996] J.P. Perdew, K. Burke and M. Ernzerhof, "Generalized Gradient Approximation Made Simple”, Physical Review Letters, Vol.77, no. 18, pp. 3865 (1996).

[Pet 1999] M. Petersen, F. Wagner, L. Hufnagel and M. Scheffler, "Improving the Efficiency of FP-LAPW Calculations”, arXiv:cond-mat/9902277 (1999).

[Pis 2012] E. Pisek, S. Rajagopal and S. Abu-Surra, "Gigabit rate mobile connectivity through visible light communication", in: Communications (ICC), 2012 IEEE International Conference on Ottawa, ON, Canada, pp. 3122-3127 (2012). 


\section{Q}

[Qin 2017] H. Qin, Y. Zuo, D. Zhang, Y. Li and J. Wu, "Received response based heuristic LDPC code for short-range non-line-of-sight ultraviolet communication," in Optics Express, Vol. 25, no. 5, pp. 5018-5030 (2017).

\section{$\mathbf{R}$}

[Ram 2002] R. Ramaswami and K. N. Sivarajan, "Optical networks: a practical perspective", The Morgan Kaufmann Series in Networking (2002).

[Rah 2011] M.B. Rahaim, AM. Vegni and T. D C. Little, "A hybrid Radio Frequency and broadcast Visible Light Communication system", in IEEE GLOBECOM Workshops (GC Wkshps), Houston, TX, USA, pp.792,796 (2011).

[Rey 2017] S. S. Reyes and A. H. Balderas, "Transmission of Digital Audio with Visible Light", in Research in Computing Science, Vol. 138, pp. 61-68 (2017).

[Ruf 2015] M. I. Ma'ruf, M. B. Othman and H. P. Sholeh, "Audio Transmission Using Visible Light Communication (VLC)", in Asian Research Publishing Network (ARPN) Journal of Engineering and Applied Sciences, Vol. 10, no. 20 (2015).

\section{$\mathbf{S}$}

[Sen 2019] U. Senkans, J. Braunfelds, I. Lyashuk, J. Porins, S. Spolitis, V. Haritonovs and V. Bobrovs, "FBG Sensors Network Embedded in Spectrum-sliced WDM-PON Transmission System Operating on Single Shared Broadband Light Source", in IEEE Photonics \& Electromagnetics Research Symposium - Fall (PIERS - Fall) (2019).

[Sek 2016] I. Sekkiou, H. Benoudnine, A. Boukortt and Y. Zidi, "Effect of oxygen substitution on the optoelectronic properties of the ternary $\mathrm{ZnSe}_{1-x} \mathrm{O}_{x}$ alloys", in Elsevier Optics communications, Vol. 127, no. 22 (2016).

[Sek 2018a] I. Sekkiou, H. Benoudnine, A. Boukortt and Y. Zidi, "Effect of oxygen substitution on the optoelectronic properties of the ternary $\mathrm{ZnSe}_{1-\mathrm{x}} \mathrm{O}_{\mathrm{x}}$ alloys", chapter for Advances in Optics: Reviews, Vol.2, Book Series, ISBN: 978-84-697-9437-1, e-ISBN: 978-84-697-9438-8, BN-20180425XX, BIC: TTB(2018).

[Sek 2018] I. Sekkiou, F. I. Chicharro, B. Ortega and J. Mora, "Bidirectional WDMOOFDM access network based on a sliceable optical transceiver with colorless ONUs", Optical Fiber Technology, Vol. 43, pp.98-105 (2018). 
[Sha 2020] M. A. Shakil Sejan, M. Habibur Rahman and W. -Y. Chung, "MPPM Based Bi-directional Long Range Visible Light Communication for Indoor Particulate Matter Monitoring”, 2020 IEEE 3rd International Conference on Computer and Communication Engineering Technology (CCET), Beijing, China, pp. 263-266 (2020).

[Sha 2006] R.A. Shafik, M.S. Rahman and A.H.M.R. Islam, "On the extended relationships among EVM, BER and SNR as performance metrics”, 4th International Conference on Electrical and Computer Engineering, pp. 408-411 (2006).

[Shi 1974] D. Schiferl, "Bonding and crystal structures of average-valence- <5> compounds: A spectroscopic approach”, Physical Review B, Vol.10, no.8, pp. 3316 (1974).

[Shi 2007] W. Shieh, H. Bao and Y. Tang. "Coherent optical OFDM: theory and design”, in Optics Express, Vol. 16, no.2, pp. 841-859 (2008).

[Shi 2006] W. Shieh and C. Athaudage, "Coherent optical orthogonal frequency division multiplexing", in Electronics Letters, Vol. 42, no. 10, pp. 587589 (2006).

[Shi 2010] W. Shieh and I. Djordjevic, "Orthogonal Frequency Division Multiplexing for Optical Communications”, in Elsevier, ISBN: 978-0-12374-879-9 (2010).

[Sin 1994] D.J.Singh, "Planewaves, pseudopotentials and the LAPW method",Kluwer Academic, Boston (1994).

[Sin 1991] D. Singh, "Ground-state properties of lanthanum: Treatment of extendedcore states”, in Physical Review B, Vol. 43, no.8, pp. 6388-6392 (1991).

[Sin 2019] A. Singh, A. Srivastava, V. A. Bohara, G. Subrahmanya and V.R.K. Rao, "Performance of hybrid cellular-VLC link for indoor environments under dynamic user movement", Physical Communication, Vol. 36, pp.100816 (2019).

[Sjö 2000] E. Sjöstedt, L. Nordström and D.J. Singh, "An alternative way of linearizing the augmented plane-wave method", Solid State Communications, Vol. 114, no.1, pp. 15-20 (2000).

[Sla 1937] J. C. Slater, "Wave Functions in a Periodic Potential", Physical Review, Vol. 51, no. 10 (1937).

[Sla 1951] J. C. Slater, "A Simplification of the Hartree-Fock Method", Physical Review, Vol. 81, no. 3 (1951).

[Ste 2002] D. Steigerwald, J. Bhat, D. Collins, R. M. Fletcher, M. Holcomb, M. Ludowise, P. Martin and S. Rudaz, "Illumination with solid state lighting technology", in IEEE Journal of Selected Topics in Quantum Electronics, Vol. 8, no. 2, pp. 310-320 (2002). 
[Sti 1972] P. J. Stiles, "Trends in the ionicity in the average valence $V$ materials", Solid State Communications, Vol. 11, no.8, pp.1063-1066 (1972).

[Sun 2008] S. B. Sun and M. S. Leeson, "Spectrum-Sliced WDM and Incoherent Optical CDMA: A Performance Comparison", Conference Proceedings, ISBN: 978-1-902560-19-9 (2008).

\section{$\mathbf{T}$}

[Tho 1927] L. H. Thomas, "The calculations of Atomic fields, Mathematical Proceedings of the Cambridge Philosophical Society, Vol. 23, no.5, pp. 542 (1927).

[Tia 2017] P. Tian, X. Liu, S. Yi, Y. Huang, S. Zhang, X. Zhou, L. Hu, L. Zheng and R. Liu, "High-speed underwater optical wireless communication using a blue GaN-based micro-LED”, in Optics Express, Vol. 25, no. 2, pp. 11931201 (2017).

[Tiw 2016] S. V. Tiwari, A. Sewaiwar, Y-H. Chung, "Uplink Bidirectional Efficient Multiuser Visible Light Communications Using TDD and Diversity Techniques", in IEEE 2016 Eighth International Conference on Ubiquitous and Future Networks (ICUFN), Vienna, Austria, pp. 225-230 (2016).

[Tsi 2014] A. Tsiatmas, C. P. M. J. Baggen, F. M. J. Willems, J. M. G. Linnartz and J. W. M. Bergmans, "An illumination perspective on visible light communications," in IEEE Communications Magazine, Vol. 52, no. 7, pp. 64-71 (2014).

\section{$\mathbf{U}$}

[Uys 2014] M. Uysal and H. Nouri, "Optical wireless communications -an emerging technology", in 16th International Conference on Transparent Optical Networks (ICTON), Graz, Austria, pp. 1-7 (2014).

\section{$\mathbf{V}$}

[Vio 2001] P. Viola and M. Jones, "Rapid object detection using a boosted cascade of simple features", Proceedings of the 2001 IEEE Computer Society Conference on Computer Vision and Pattern Recognition. CVPR 2001, Kauai, HI, USA, pp. I-I (2001).

[Vuc 2009a] J. Vucic, C. Kottke, S. Nerreter, K. Habel, A. Buttner, K.-D. Langer and J. W. Walewski , J., "125 Mbit/s over $5 \mathrm{~m}$ wireless distance by use of OOK-Modulated phosphorescent white LEDs", 2009 35th European Conference on Optical Communication, Vienna, Austria, 2009, pp. 1-2 
(2009).

[Vuc 2009b] J. Vucic, C. Kottke, S. Nerreter, A. Buttner, K. -. Langer and J. W. Walewski, "White Light Wireless Transmission at 200+ Mb/s Net Data Rate by Use of Discrete-Multitone Modulation", in IEEE Photonics Technology Letters, Vol. 21, no. 20, pp. 1511-1513 (2009).

[Vuc 2010] J. Vucic, C. Kottke, S. Nerreter, K. Habel, A. Büttner, K-. Langer and J. W. Walewski, "230 Mbit/s via a wireless visible-light link based on OOK modulation of phosphorescent white LEDs", 2010 Conference on Optical Fiber Communication (OFC/NFOEC), collocated National Fiber Optic Engineers Conference, San Diego, CA, USA, pp. 1-3 (2010).

\section{W}

[Wan 2017] Z. Wang, Q. Wang, W. Huang and Z. Xu, "Visible Light Communications: Modulation and Signal Processing”,Wiley-IEEE Press, ISBN: 978-1-119-33138-4 (2017).

[Wan 2015] Z. Wang, D. Tsonev, S. Videv and H. Haas, "On the Design of a SolarPanel Receiver for Optical Wireless Communications With Simultaneous Energy Harvesting", in IEEE Journal on Selected Areas in Communications, Vol. 33, no. 8, pp. 1612-1623 (2015).

[Wan 2013] Y. Wang, M. Alharbi, T. D. Bradley ,C. Fourcade-Dutin ,B. Debord , B. Beaudou , F. Gerôme and F. Benabid, "Hollow-core photonic crystal fibre for high power laser beam delivery”, High Power Laser Science and Engineering, Vol. 1, no. 1, pp. 17 - 28 (2013).

[Wat 2013] S. Watson, M. Tan, S. P. Najda, P. Perlin, M. Leszczynski, G. Targowski, S. Grzanka, and A. E. Kelly, "Visible light communications using a directly modulated $422 \mathrm{~nm}$ GaN laser diode", Optics Letters, Vol. 38, no. 19, pp. 3792-3794 (2013).

[Wei 1971] S.B. Weinstein and P.M. Ebert, "Data transmission by frequency-division multiplexing using the discrete Fourier transform", in IEEE Transactions on Communication Technology, Vol. 19, no. 5, pp. 628-634 (1971).

[Wei 2009] J. L. Wei, X. Q. Jin and J. M. Tang, "The influence of directly modulated DFB lasers on the transmission performance of carrier suppressed singlesideband optical OFDM signals over IMDD SMF systems", in Journal of Lightwave Technology, vol. 27, no. 13, pp. 2412-2419, Vol. 27, no. 13, pp. 2412-2419 (2009).

[Won 2000] K. Wong and T. O'Farrell, "Infrared wireless communications", in 11th IEEE International Symposium on Personal Indoor and Mobile Radio Communications. PIMRC 2000, Proceedings (Cat. No.00TH8525), 
London, UK, Vol.2, pp. 933-938 (2000).

[Wu 2011] D. Wu, Z. Ghassemlooy, H. LeMinh, S. Rajbhandari and Y.S. Kavian, "Power Distribution and Q-factor Analysis of Diffuse Cellular Indoor Visible Light Communication Systems", in 2011 16th European Conference on Networks and Optical Communications, Newcastle upon Tyne, UK, pp. 28-31 (2011).

\section{$\mathbf{X}$}

[Xu 2008] Z. Xu and B.M. Sadler, "Ultraviolet communications: potential and stateof-the-art, in IEEE Communications Magazine, Vol. 46, no. 5, pp. 67-73 (2008).

\section{$\mathbf{Y}$}

[Yeh 2019] C-H. Yeh, J-H. Weng, C-W. Chow, C-M. Luo, Y-R. Xie, C-J. Chen and M-C. Wu, "1.7 to 2.3 Gbps OOK LED VLC Transmission Based on $4 \times 4$ Color-Polarization-Multiplexing at Extremely Low Illumination", in IEEE Photonics Journal, Vol. 11, no. 4, pp. 1-6 (2019).

[Yos 2015] R. Yoshizawa and H. Ochiai, "Effect of Clipping and Filtering with Distortion less PAPR Reduction for OFDM Systems", 2015 IEEE 82nd Vehicular Technology Conference (VTC2015-Fall), Boston, MA, USA, 2015, pp. 1-5 (2015).

[You 2015] X. You, J. Chen, H. Zheng and C. Yu, "Efficient Data Transmission Using MPPM Dimming Control in Indoor Visible Light Communication", in IEEE Photonics Journal, Vol. 7, no. 4, pp. 1-12 (2015).

\section{$\mathbf{Z}$}

[Zha 2020] P. Zhao, K. Ma, Z. Wang and S. Chen, "Virtual Angular-Domain Channel Estimation for FDD Based Massive MIMO Systems with Partial Orthogonal Pilot Design", in IEEE Transactions on Vehicular Technology, vol. 69, no. 5, pp. 5164-5178 (2020).

[Zha 2018] Z. Zhang, T. Zhang, J. Zhou, Y. Lu and Y. Qiao, "Mobile phone Camera Based Visible Light Communication Using Non-Line-of-Sight (NLOS) Link", in 2018 International Conference on Network Infrastructure and Digital Content (IC-NIDC), Guiyang, China, pp. 35-39 (2018).

[Zha 2016] Q. Zhang, H. Dun, C. Qian, B. Cao, M. Mao, J. Zhang and M. Wang, "Experimental Demonstration of 7.09Gb/s DML based Real-time Optical 
OFDM Transmission with Spectral Efficiency up to $6.93 \mathrm{bits} / \mathrm{s} / \mathrm{Hz}$ over 50km SSMF", in 2016 21st OptoElectronics and Communications Conference (OECC) held jointly with 2016 International Conference on Photonics in Switching (PS), Niigata, Japan, pp. 1-3 (2016).

[Zho 2019] H. Zhou, M. Zhang, X. Wang, Y. Sun, Y. Zhang, C. Wei, W. He, W. Sun, C. Qin, C. Guan and X. Ren, "120m 10Mbps Ethernet Transmission Based on Visible Light Communication using a Single Commercially Available LED”, in 2019 18th International Conference on Optical Communications and Networks (ICOCN), Huangshan, China, pp. 1-3 (2019).

[Zhu 2017] Y.-J. Zhu, Z.-G. Sun, J.-K. Zhang, Y.-Y. Zhang, and J. Zhang, “Training receivers for repetition-coded MISO outdoor visible light communications", in IEEE Transactions on Vehicular Technology, vol. 66, no. 1, pp. 529-540 (2017).

[Zyg 2010] L. Zyga. (2010). https://phys.org/news/2010-01-siemens-wirelesswhite.html, http://www.siemens.com/innovation/en/news/2010/500megabits-second-with-white-led-light.htm. 
Appendixes 


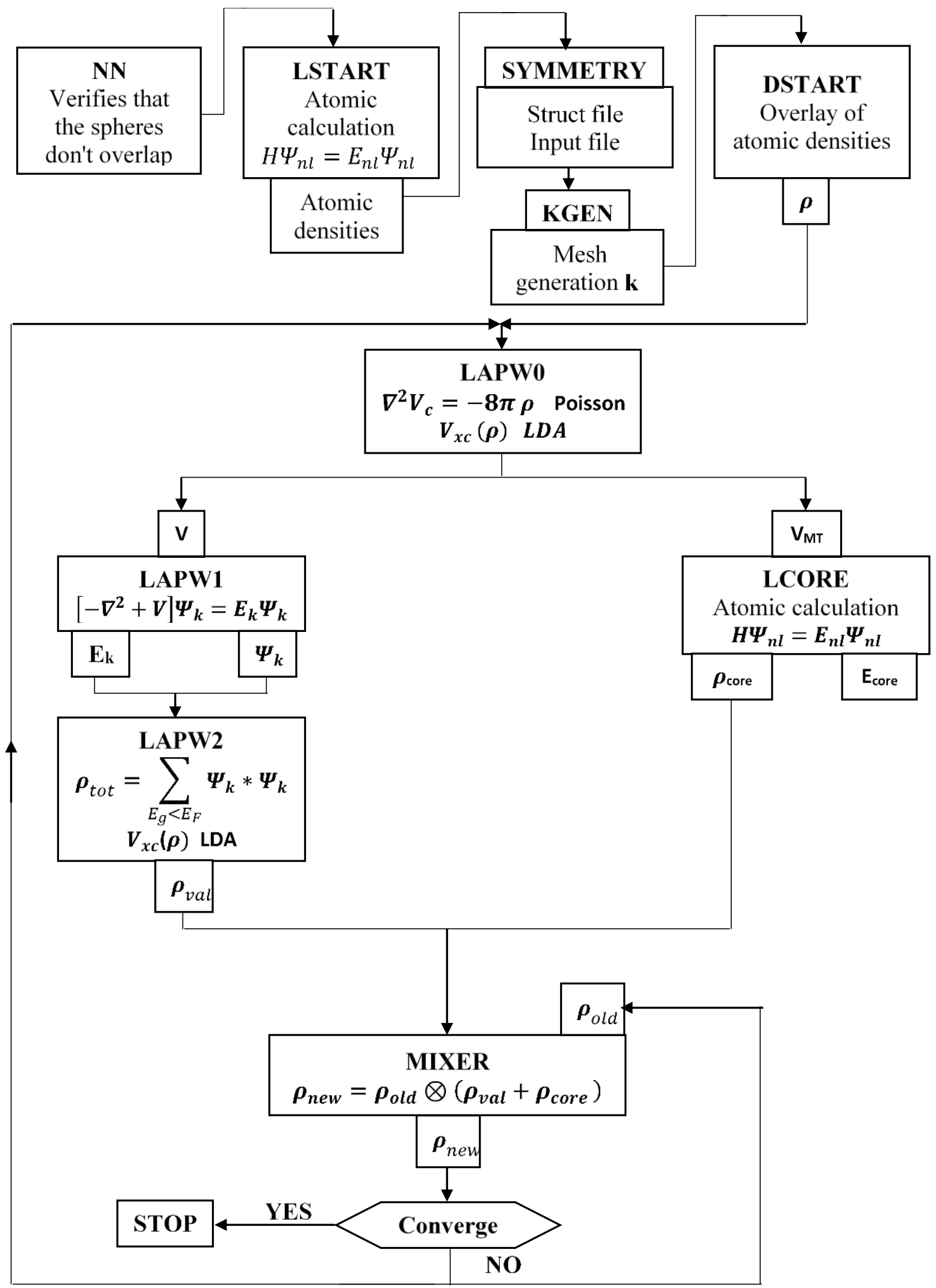



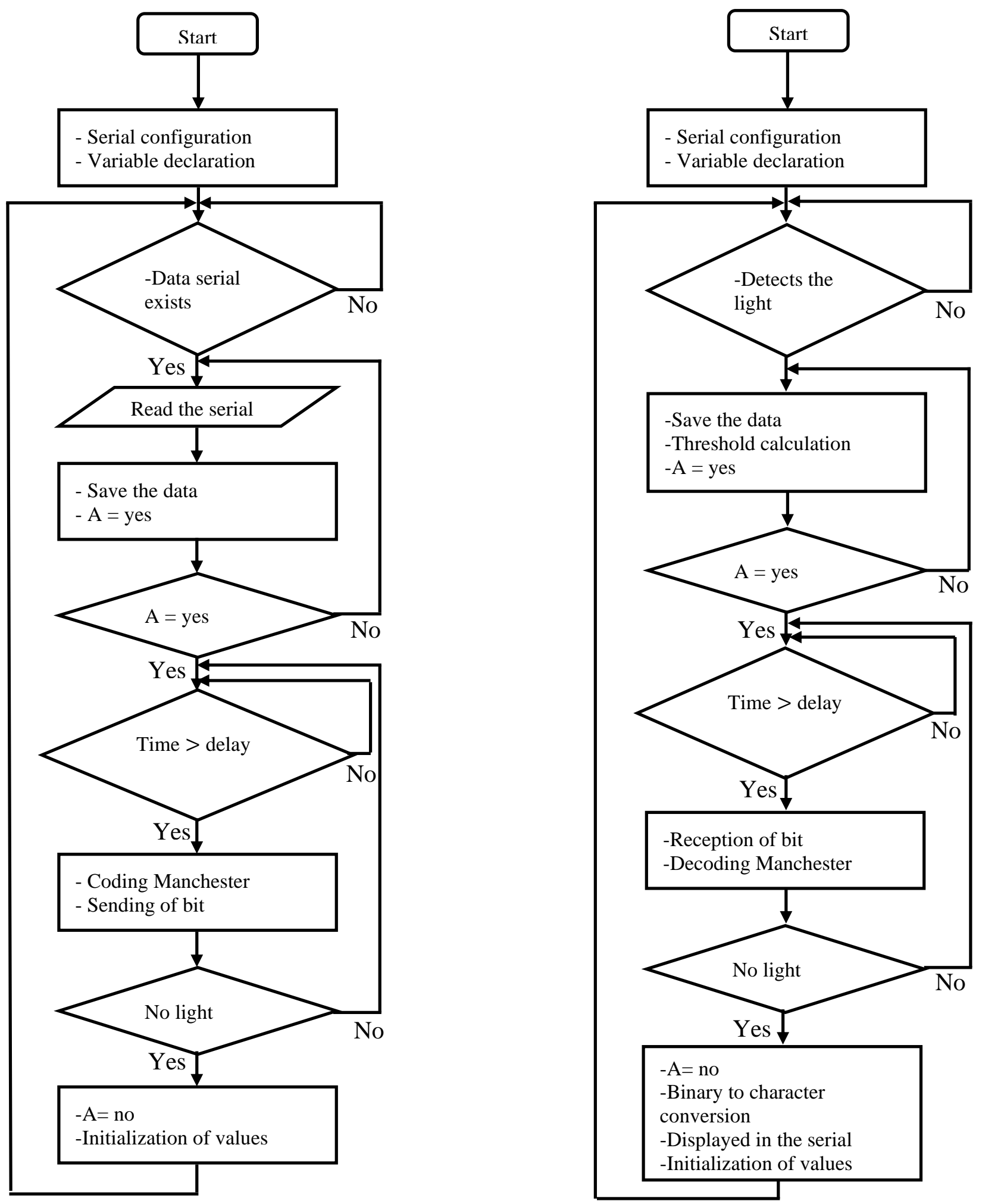

Emission diagram

Reception diagram 

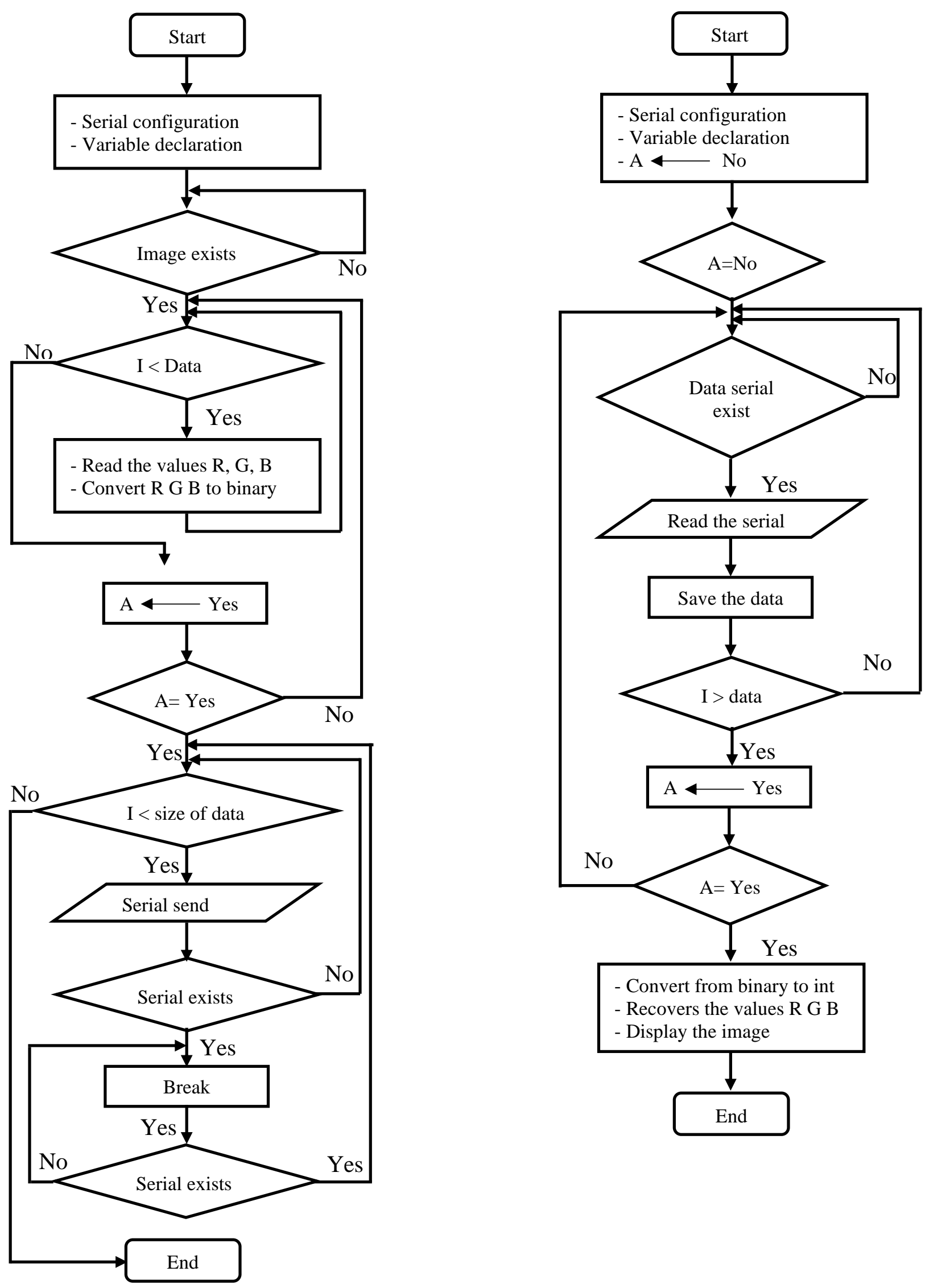

Emission diagram

Reception diagram 


\section{A. Publications}

- [Sek 2018] Imene Sekkiou, Francisco I. Chicharro, Beatriz Ortega, José Mora, 'Bidirectional WDM-OOFDM access network based on a sliceable optical transceiver with colorless ONUs', Optical Fiber Technology 45 (2018) 98-105.

- [Sek 2016] Sekkiou, H. Benoudnine, A. Boukortt, Y. Zidi, 'Effect of oxygen substitution on the optoelectronic properties of the ternary $\mathrm{ZnSe}_{1-\mathrm{x}} \mathrm{O}_{\mathrm{x}}$ alloys,' Optik International Journal for Light and Electron Optics, Elseiver Optik 127 (2016) 1102011028.

\section{B. Chapter Book}

- Luis Felipe Albarracín, Imene Sekkiou, Beatriz Ortega, Francisco Chicharro, José Mora et Gustavo Puerto, 'Software assisted energy efficient and flexible WDM- PON access networks', chapter for 'Communications in Computer and Information Science', Springer, Cham, Vol.915, book series, ISBN: 978-3-030-00349-4, Online ISBN: 978-3-030-00350-0, 2018.

- I. Sekkiou, H. Benoudnine, A. Boukortt, Y. Zidi, 'Effect of oxygen substitution on the optoelectronic properties of the ternary $\mathrm{ZnSe}_{1-\mathrm{x}} \mathrm{O}_{\mathrm{x}}$ alloys,' chapter for 'Advances in Optics: Reviews', Vol. 2, Book Series, ISBN: 978-84-697-9437-1, e-ISBN: 978-84697-9438-8, 2018.

\section{Conferences:}

- Luis Felipe Albarracín, Imene Sekkiou, Beatriz Ortega, Francisco Chicharro, José Mora and Gustavo Puerto, 'Software assisted energy efficient and flexible WDMPON access networks', Workshop on engineering Applications (WEA '18), Springer, Cham, pp 588-595, ISBN: 978-3-030-00349-4.

- I. Sekkiou,T. Bouzid, Z.Hamdi, H.Benoudnine, ' Wireless Communication using LEDS', Fouth Scientific workshop of the PHD studies, Technology and Faculty of Science, University Abdelhamid Ibn Badis, Mostaganem, Algeria, April 28th, 2016.

- I.Sekkiou, H.Benoudnine, M.Dahmani et R.Benzidane, ' Free Space Optical Data transmission for long distance wireless communication,' Innovation Connecting Show, ICS 2014, 16-18 september 2014, Toulouse, France.

- I. Sekkiou, H.Benoudnine et A.Boukortt, 'Study of the electric \& optical behavior of LED manufactured containing GaN `, Third Scientific workshop of the PHD studies, Technology and Faculty of Science, University Abdelhamid Ibn Badis, Mostaganem, Algeria May 19th, 2014.

\section{Other merits}

- Scholarship (1 year: 19/09/2016 to 31/07/2017), PHD in Telecommunication, at Universitat Politècnica de València Spain.

- Practical Training (1 month: 15/09/2015 to 18/10/2015), at LISV - Versailles Engineering Systems Laboratory, Paris, France. 
- Temporary Teacher (2015-2016) given Tutorials in Mathematics for Chemistry Bachelor program (Level 1), Physics Department, Faculty of Chemistry, University of Sciences and Technologies of Oran, USTO, Mohammed Boudiaf, Algeria.

- Temporary Teacher (2014-2015) as responsible of Networks and Databases Courses and Practical works (lab works), Chemistry Bachelor program (level 3), Physics Department, Faculty of Chemistry, University of Sciences and Technologies of Oran, USTO, Mohammed Boudiaf, Algeria.

- Temporary Teacher (2013-2014) as responsible of Data Processing courses and practical works and Tutorials in Mathematics for level 3 Chemistry Bachelor program, Physics Department, Faculty of Chemistry, University of Sciences and Technologies of Oran, USTO, Mohammed Boudiaf, Algeria. 